\title{
Análise de questionários com itens constrangedores
}

\section{Mariana Cúri}

\author{
Tese apresentada ao \\ Instituto de Matemática e Estatística \\ da Universidade de São Paulo \\ para obtenção do grau de \\ Doutor em Estatística
}

\section{Área de concentração: Estatística \\ Orientador: Prof. Dr. Julio da Motta Singer}

Durante a elaboração deste trabalho

o autor recebeu apoio financeiro da CAPES

- São Paulo, Agosto de 2006 - 


\section{ANÁLISE DE QUESTIONÁRIOS COM ITENS CONSTRANGEDORES}

Este exemplar corresponde à redação final da tese devidamente corrigida e defendida por Mariana Cúri e aprovada pela comissão julgadora.

São Paulo, 11 de Agosto de 2006.

Comissão Julgadora:

- Prof. Dr. Julio da Motta Singer (Orientador) - IME-USP

- Prof. Dr. Dalton Francisco de Andrade - INE-UFSC

- Prof. Dr. Enrico Antônio Colosimo - UFMG

- Prof. Dr. Héliton Ribeiro Tavares - UFPA

- Prof. Dr. Ruben Klein - LNCC-CNPq 
"- As coisas que vemos - continuou Pistórius com uma voz mais velada - são as mesmas que temos dentro de nós. A única realidade é aquela que se contém dentro de nós, e se os homens vivem tão irrealmente é porque aceitam como realidade as imagens exteriores e sufocam em si a voz do mundo inteiro. Também se pode ser feliz assim; mas quando se chega a conhecer o outro, torna-se impossivel seguir o caminho da maioria."

Herman Hesse, em Demian 


\section{Agradecimentos}

De todo o conteúdo que vem nas páginas e páginas a seguir, este é o que escrevo com

mais leveza. É o que me faz recordar de quando surgiu a vontade de iniciar este trabalho longo, árduo e, como não poderia negar, prazeroso também.

Retorno ao ano 2000, quando ainda trabalhava no InCor, acompanhada de minhas queridas amigas Lilian e Carine e de meu amado companheiro Clovis. Ouço-nos a confabular maneiras de driblar e vencer a disfarçada resistência ao nosso desejo de percorrer este caminho. A solução estava por perto, do outro lado da rua, num outro canto de quarteirão. Amparada por eles, pelos meus pais, Sílvia e Cúri, irmãos, Márcia e Maurício, e cunhado, Fred, atravessei a tal rua... E desci a rampa, indo desaguar nos braços psiquiátricos do IPq. Pude, então, iniciar esta vereda árdua, juntamente com minha outra querida amiga, Raquel.

Sigo a desbravar os dois lados de paisagem: o psíquico e o exato. Conhecendo o toc das fobias, de agora, das febres... a passar pela coréia, pelos dois pólos, numa hiperatividade de transtornar todas as personalidades e concentrar-me numa, enfim, que mente, constrange, desconcerta e leva a mente, livre das amarras, a aterrizar na resposta das teorias já ditas, há algum tempo atrás.

Traz-me à lembrança os encontros de República, fundada em Chagas, nas companhias das amigas Lilian e Raquel, sempre presentes, e dos "mestres" Dalton e Bussab.

Foi assim que, depois de mais da metade do caminho percorrido, mudei o rumo. Sempre apoiada e, especialmente, guiada pelo meu (há tanto tempo) orientador Julio... Ao Julio e ao meu co-orientador (infelizmente, extra-oficial), Dalton, gostaria de agradecer muitíssimo pela ajuda, paciência e ensinamentos preciosos transmitidos nesses últimos anos.

Aos psiquiatras Laura e Wang, agradeço as discussões sobre interpretação e adequação dos modelos estatísticos à Psiquiatria. Ainda a eles e aos seus colegas Clarice e Dartiu, 
agradeço a gentil cessão dos conjuntos de dados.

Aos colegas Caio, Héliton, Reiko, Marinho, Mário, Vicente e João Batista, sou grata pela ajuda nas etapas de estimação, proposição do modelo, conferência das fórmulas no Mathematica, estimação pelo MCMC, derivação das expressões, programação no WinBUGS e inserção das figuras no Latex, respectivamente.

De novo ao Clovis, que ainda trouxe o fôlego da localização do ponto final, para continuar depois... A ele, a meus pais, irmãos e amigas Ângela, Maria Paula, Lilian, Carine, Raquel, Camila e Renata, pelo prazer de dividir as alegrias e pelas palavras certeiras, nos momentos de desânimo.

Foi realmente com muita persistência e participação de todos vocês que consegui depositar esta tese no dia 28 de abril de 2006. 


\section{Resumo}

As pesquisas científicas na área da Psiquiatria freqüentemente avaliam características subjetivas de indivíduos como, por exemplo, depressão, ansiedade e fobias. Os dados são coletados através de questionários, cujos itens tentam identificar a presença ou ausência de certos sintomas associados à morbidade psiquiátrica de interesse. Alguns desses itens, entretanto, podem provocar constrangimento em parte dos indivíduos respondedores por abordarem características ou comportamentos socialmente questionáveis ou, até, ilegais. Um modelo da teoria de resposta ao item é proposto neste trabalho visando diferenciar a relação entre a probabilidade de presença do sintoma e a gravidade da morbidade de indivíduos constrangidos e não constrangidos. Itens que necessitam dessa diferenciação são chamados itens com funcionamento diferencial. Adicionalmente, o modelo permite assumir que indivíduos constrangidos em responder um item possam vir a mentir em suas respostas, no sentido de omitir a presença de um sintoma. Aplicações do modelo proposto a dados simulados para questionários com 20 itens mostraram que as estimativas dos parâmetros são próximas aos seus verdadeiros valores. A qualidade das estimativas piora com a diminuição da amostra de indivíduos, com o aumento do número de itens com funcionamento diferencial e, principalmente, com o aumento do número de itens com funcionamento diferencial suscetíveis à mentira. A aplicação do modelo a um conjunto de dados reais, coletados para avaliar depressão em adolescentes, ilustra a diferença do padrão de resposta do item "crises de choro" entre homens e mulheres. 


\section{Abstract}

In psychiatric scientific research subjective characteristics of the individual such as depression, anxiety and phobias are often evaluated. Data are collected through questionnaires with items in an attempt to identify the presence of certain symptoms associated with some psychiatric disease. Some of these items may embarass the respondents since they are related to questionable or even illegal social behaviors. An Item Response Theory model is proposed to differentiate the relationship between the probability of the presence of the symptom and the gravity of the disease of embarrassed and non-embarrassed individuals. Items that need this differentiation are called differential item functioning (DIF). Additionally, the model assumes that individuals embarrassed with one particular item could lie across other answers to omit a possible condition. Applications of the proposed model to simulated data for a 20-item questionnaire show that parameter estimates of the proposed model are close to their real values. The accuracy in estimation gets worse as the number of individuals decreases, the number of DIF increases, and especially as the number of DIF susceptible to lying increases. An example with real data, collected to evaluate teenager depression, highlights the difference in the probability of "crying crisis" for men and women. 


\section{Sumário}

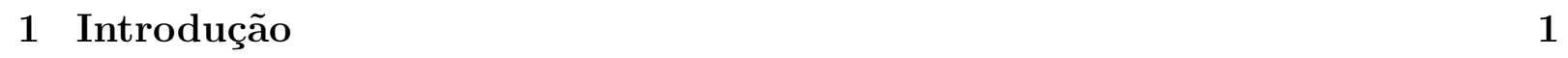

1.1 Motivação . . . . . . . . . . . . . . . . . . . . . . 2

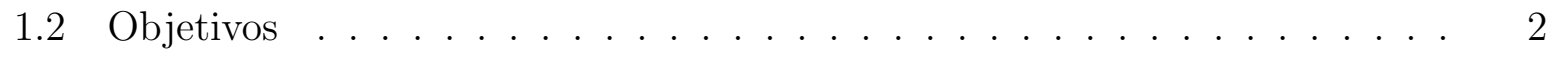

1.3 Caracterização dos Instrumentos de Avaliação . . . . . . . . . . . . . . . . 3

\begin{tabular}{|ll|}
\hline 1.4 Métodos Clássicos para o Estudo da Qualidade de Instrumentos de Avaliação \\
\hline Psiquiátrica
\end{tabular}

1.5 Vantagens da TRI para o Estudo da Qualidade de Instrumentos de Avaliação

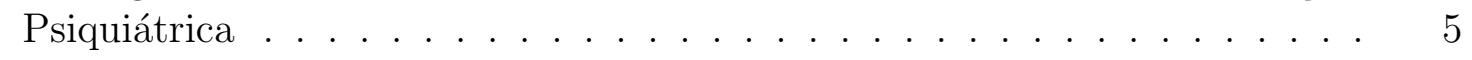

\begin{tabular}{|lll}
\hline 2 & Metodologia da TRI & 7 \\
\hline
\end{tabular}

2.1 Principais Modelos da TRI . . . . . . . . . . . . . . . . . . . . . 7

2.2 Métodos de Estimação . . . . . . . . . . . . . . . . . . . . . . . 14

2.3 Aspectos Computacionais . . . . . . . . . . . . . . . 18

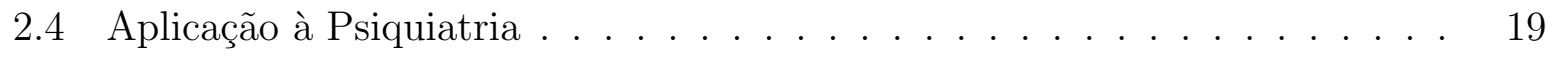

$2.4 .1 \quad$ Aplicação do ML3 a um conjunto de dados psiquiátricos . . . . . . 20

2.4 .2 Comparação entre a teoria clássica e o ML3 da TRI no estudo da qualidade de instrumentos de avaliação psiquiátrica . . . . . . . . 23

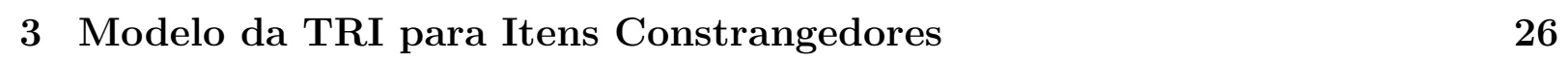

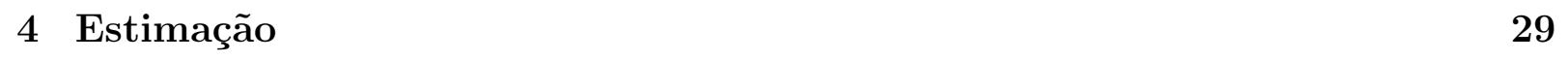

4.1 Itens Conhecidos - Máxima Verossimilhança (MV) . . . . . . . . . . . . . . 29 
4.2 Itens e Habilidades Desconhecidos - MV Marginal (MVM) $\ldots . . . . . . .32$

4.2 .1 Estimação de $\boldsymbol{\zeta}_{i}$ para todos os itens simultaneamente . . . . . . . . 32

$4.2 .2 \quad$ Estimação de $\boldsymbol{\zeta}_{i}$ para os itens individualmente . . . . . . . . . . . . 41

$4.3 \quad$ Itens e Habilidades Desconhecidos - Monte Carlo via Cadeia de Markov

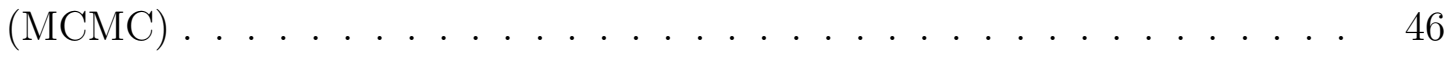

\begin{tabular}{|lll}
5 & Exemplos com Amostras Simuladas & 48
\end{tabular}

6 Aplicação a Dados Psiquiátricos Reais 63

\begin{tabular}{llc}
\hline 7 & Estudos Futuros & 69
\end{tabular}

\begin{tabular}{|ll}
\hline A Expressões Detalhadas & 71
\end{tabular}

\begin{tabular}{ll}
\hline B Escalas Psiquiátricas & 85
\end{tabular}

B.1 Inventário de Depressão de Beck (BDI) … . . . . . . . . . . . . . 85

B.2 Escala de Sintomas Depressivos (CES-D) … . . . . . . . . . . . . 88

B.3 Escala de Rastreamento de Dependência de Sexo (ERDS) . . . . . . . . . . 89

B.4 Teste de Investigação Bulímica de Edinburgh (TIBE) . . . . . . . . . . . . 90

B.5 Inventário de Triagem do Uso de Drogas Reformulado (DUSI-R) . . . . . . 92 


\section{Lista de Tabelas}

2.1 Parâmetros do ML3 ajustados para os dados do BDI de Wang et al. (2005) 22

5.1 Cenário utilizado para a geração das amostras simuladas para um questionário com 20 itens. . . . . . . . . . . . . . . . . . . . . . . . . . . 49

5.2 Valores fixados para os parâmetros de item para a simulação das respostas. (a: parâmetro de discriminação; $b_{1}$ : parâmetro de gravidade de depressão para indivíduos não constrangidos; $b_{2}$ : parâmetro de gravidade de depressão para indivíduos constrangidos; $\gamma$ : probabilidade de um indivíduo constrangido admitir que tem o sintoma; $\delta:$ probabilidade de o item constranger o indivíduo) . . . . . 50

5.3 Estimativas dos parâmetros $b_{2 i}, \gamma_{i}$ e $\delta_{i}$, para $i=1,2$, nas simulações com 1 e

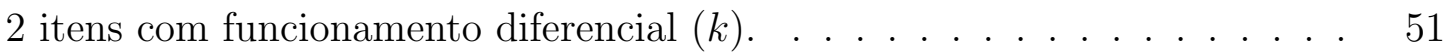

5.4 Valores médios, mínimos, medianos, máximos e percentis 75 (P75) dos desvios dos estimadores dos parâmetros. . . . . . . . . . . . . . . . . . 61

6.1 Parâmetros de item do modelo proposto ajustados para os dados de depressão de CES-D. . . . . . . . . . . . . . . . . 65 


\section{Lista de Figuras}

$2.1 \quad$ Exemplo de curva característica de um item sob o ML3 . . . . . . . . . . 8

2.2 CCI de dois itens sob o ML2 . . . . . . . . . . . . . . . . . . . . . 10

2.3 CCI de dois itens sob o ML1 - modelo de Rasch . . . . . . . . . . . . . . . 11

2.4 CCI de um item sob o ML4 . . . . . . . . . . . . . . . . . . . . . . . . . . 11

2.5 Probabilidade acumulada da escolha de cada categoria de um item sob o modelo de Samejima . . . . . . . . . . . . . . . . . . . . . . . 12

2.6 Probabilidade da escolha de cada categoria de um item sob o modelo de

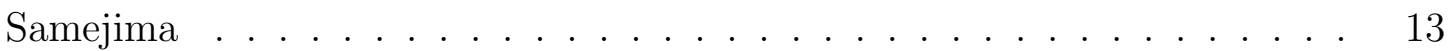

2.7 CCI dos 21 itens do BDI com parâmetros obtidos do ajuste de um ML3 para os dados de Wang et al. (2005). . . . . . . . . . . . . . . . . . . 21

2.8 Estimativa dos parâmetros de gravidade (b) e probabilidade de presença de sintoma em indivíduos sem depressão (c) dos 21 itens do BDI ajustados pelo ML3 para os dados de Wang et al. (2005). (*: itens âncora no nível $\theta=1$; **: itens âncora no nível $\theta=2 \mathrm{e}^{* * *}$ : itens âncora no nível $\left.\theta=3\right) . . . .24$

5.1 Gráficos de dispersão entre os valores verdadeiros e estimados dos parâmetros na simulação para $n=300$ indivíduos e $\mathrm{k}=1$ item com funcionamento diferencial ((a)-(c)) e para $\mathrm{k}=2$ itens com funcionamento diferencial $((\mathrm{d})-(\mathrm{f}))$. (r: coeficiente de correlação de Pearson) $\ldots$. . . . . . . . . . . . . . . . . 53

5.2 Gráficos de dispersão entre os valores verdadeiros e estimados dos parâmetros na simulação para $n=300$ indivíduos e $k=6$ itens com funcionamento diferencial. (r: coeficiente de correlação de Pearson) . . . . . . . . . . . . . . . . . 54

5.3 Gráficos de dispersão entre os valores verdadeiros e estimados dos parâmetros na simulação para $n=500$ indivíduos e $\mathrm{k}=1$ item com funcionamento diferencial ((a)-(c)) e para $\mathrm{k}=2$ itens com funcionamento diferencial $((\mathrm{d})-(\mathrm{f})) .(\mathrm{r}$ : coeficiente de correlação de Pearson) $\ldots$. . . . . . . . . . . . . . . . . . 55 
5.4 Gráficos de dispersão entre os valores verdadeiros e estimados dos parâmetros na simulação para $\mathrm{n}=500$ indivíduos e $\mathrm{k}=6$ itens com funcionamento diferencial. (r: coeficiente de correlação de Pearson) $\ldots$. . . . . . . . . . . . . . . . 56

5.5 Gráficos de dispersão entre os valores verdadeiros e estimados dos parâmetros na simulação para $\mathrm{n}=500$ indivíduos e $\mathrm{k}=10$ itens com funcionamento diferencial. (r: coeficiente de correlação de Pearson) $\ldots$. . . . . . . . . . . . . . . . 57

5.6 Gráficos de dispersão entre os valores verdadeiros e estimados dos parâmetros na simulação para $n=1000$ indivíduos e $k=6$ itens com funcionamento diferencial. (r: coeficiente de correlação de Pearson) $\ldots$. . . . . . . . . . . . . . . . 58

5.7 Gráficos de dispersão entre os valores verdadeiros e estimados dos parâmetros na simulação para $\mathrm{n}=1000$ indivíduos e $\mathrm{k}=10$ itens com funcionamento diferencial. (r: coeficiente de correlação de Pearson)

5.8 Gráficos de dispersão entre os valores verdadeiros e estimados dos parâmetros na simulação para $n=1000$ indivíduos e $\mathrm{k}=20$ itens com funcionamento diferencial. (r: coeficiente de correlação de Pearson) $\ldots$. . . . . . . . . . . . . . . . 60

5.9 Gráficos dos desvios médios das estimativas dos parâmetros. (n: número de indivíduos; k: número de itens com funcionamento diferencial) ] . . . . . . . . . 62

6.1 Estimativas dos parâmetros de (a) discriminação e (b) gravidade dos dados de depressão de CES-D. . . . . . . . . . . . . . . . . . . . . 66

6.2 CCI choro para indivíduos do sexo masculino (M), feminino (F) e sem especificação de gênero, estimada para os dados de depressão de CES-D. . . . 67

6.3 Níveis de depressão individuais estimados para os dados da CES-D. . . . . 67

6.4 Boxplot do nível de depressão individual estimado para os diagnósticos de depressão baseados no escore total da CES-D. . . . . . . . . . . . . . . 68 


\section{Lista de Abreviações}

BDI: inventário de depressão de Beck

CCI: curva característica do item

CES-D: escala de sintomas depressivos

DUSI: inventário de triagem do uso de drogas

DUSI-R: inventário de triagem do uso de drogas reformulado

EAP: esperança a a posteriori

ERDS: escala de rastreamento de dependência de sexo

MAP: moda a a posteriori

MCMC: monte carlo via cadeia de Markov

ML1: modelo logístico unudumensional de um parâmetro

ML2: modelo logístico unudumensional de dois parâmetros

ML3: modelo logístico unudumensional de três parâmetros

ML4: modelo logístico unudumensional de quatro parâmetros

MV: máxima verossimilhança

TIBE: teste de investigação bulímica de Edinburgh

TRI: teoria de resposta ao item 


\section{Capítulo 1}

\section{Introdução}

A pesquisa científica em áreas como Psicologia, Educação, Psiquiatria, Marketing, entre outras, tem como principal obstáculo a quantificação do construto (aqui visualizado como traço latente não observável) que se deseja estudar. Para tanto, o procedimento mais utilizado é a elaboração de um instrumento de medida (ou instrumento de avaliação) que consiste na operacionalização do construto por meio de sua especificação em itens. Por exemplo, a prova (instrumento de avaliação) do vestibular quantifica o conhecimento dos candidatos (construto) através de testes de múltipla escolha (itens) cujo conteúdo avalia diferentes tópicos das disciplinas do ensino fundamental e médio. Esse instrumento de medida pode receber diferentes denominações, como: escala, inventário, questionário ou prova. Uma das grandes preocupações dos pesquisadores dessa área é elaborar um instrumento de avaliação que quantifique fidedignamente o construto de interesse. Neste contexto, foram criados alguns critérios para elaboração desses instrumentos e desenvolvidas técnicas estatísticas adequadas à avaliação de sua capacidade de representar o construto, que serão mencionadas nas seções seguintes. Este trabalho pretende apresentar a Teoria de Resposta ao Item (TRI) como um método estatístico para a avaliação da qualidade do instrumento, na área de Psiquiatria.

As duas seções subsequentes apresentam a motivação e os objetivos específicos desta tese. O restante do capítulo é dedicado a um breve relato sobre a elaboração de instrumentos psiquiátricos. Em particular, a seção 1.3 descreve as principais características de um instrumento de avaliação. A seção 1.4 apresenta os métodos clássicos mais comumente utilizados na avaliação da qualidade dos instrumentos na área de Psiquiatria. A seção 1.5 menciona as vantagens que a TRI pode ter sobre esses métodos. No capítulo 2, são apresentados alguns modelos mais comuns da TRI para problemas oriundos das áreas educacional e psiquiátrica, além dos principais métodos de estimação de seus parâmetros. O capítulo é finalizado com uma aplicação a um conjunto de dados reais de pacientes com transtorno depressivo. No capítulo 3, uma modificação de um dos modelos da TRI 
é proposta com a finalidade de adaptar a teoria às peculiaridades de alguns instrumentos psiquiátricos. No capítulo 4, obtêm-se os respectivos estimadores dos parâmetros. No capítulo 5, encontram-se descritas simulações realizadas com o intuito de avaliar a utilidade do modelo proposto. Finalmente, no capítulo 6, o modelo proposto é aplicado a um conjunto de dados psiquiátricos reais.

\subsection{Motivação}

Alguns instrumentos psiquiátricos têm peculiaridades que não são contempladas pelos modelos existentes da TRI. Instrumentos desenvolvidos para avaliação de psicoses, uso e abuso de álcool e drogas, transtornos alimentares e de personalidade são exemplos disso. Compostos por itens que avaliam a presença de características socialmente questionáveis, ilegais ou consideradas vergonhosas pelo próprio indivíduo, são muito suscetíveis a falsas respostas. Cappaliez (1989) mostra que alguns sintomas depressivos são minimizados por respostas que têm maior aceitação social. De uma maneira geral, os psiquiatras relatam uma maior freqüência de respostas falso-negativas a itens que avaliam idéias suicidas ou choro (especialmente nos homens), a prática de roubo, estupro ou assalto, consumo de drogas ilícitas, comportamentos sexuais considerados obscenos ou imorais, escuta de vozes, percepção corporal (relacionada à beleza, gordura, flacidez), hábitos alimentares (jejuar, comer sem parar, ingerir laxantes, diuréticos ou provocar vômito para perder peso), etc. Itens desse tipo podem ser encontrados nos instrumentos apresentados no Apêndice B: Inventário de Depressão de Beck (BDI), Escala de Rastreamento de Dependência de Sexo (ERDS), Teste de Investigação Bulímica de Edinburgh (TIBE), Escala de Sintomas Depressivos (CES-D) e inventário de triagem do uso de drogas (DUSI). Um projeto desenvolvido no Instituto de Psiquiatria do Hospital das Clínicas da USP com o objetivo de estudar as propriedades psicométricas e a validade transcultural do BDI em uma amostra de 208 imigrantes chineses residentes em São Paulo (Wang, Andrade \& Gorenstein 2005) foi a alavanca da proposta desta tese. O BDI é um instrumento de medida que não tem por objetivo diagnosticar a depressão, mas sim medir a gravidade dos sintomas depressivos

por meio de 21 itens que avaliam a intensidade de diferentes sintomas da doença (vide Apêndice B).

\subsection{Objetivos}

Uma das possíveis contribuições desta tese é a disseminação da TRI no Brasil como um método de estudo das propriedades psicométricas de instrumentos de avaliação de transtornos psiquiátricos. O cerne deste trabalho é propor um modelo da TRI que leve em consideração que alguns itens do instrumento podem constranger um grupo de indivíduos, 
diferenciando seus comportamentos dos demais e incorporando a possível existência de falsas respostas (mais especificamente, respostas mentirosas ou incongruentes). Sob o enfoque da TRI, o modelo visa acomodar itens com funcionamento diferencial (Camilli \& Shepard 1994).

\subsection{Caracterização dos Instrumentos de Avaliação}

A elaboração de um instrumento de avaliação envolve diferentes características como o número de itens, o tipo de item (fechado ou aberto), o número de categorias de resposta dos itens fechados, o método de aplicação (auto-aplicação ou aplicação por meio de um entrevistador), o número de traços latentes e o número de dimensões de cada traço latente que devem compô-lo. Não existe um padrão mais adequado para essas características e, por isso, diferentes tipos de instrumentos podem ser encontrados e formulados, dependendo da situação. Na primeira fase da FUVEST, a prova é composta por 100 itens com 5 alternativas de resposta que avaliam vários traços latentes (conhecimento sobre Matemática, Português, História, Geografia, Física, Química, Inglês e Biologia) e são respondidos pelo próprio indivíduo, sem a necessidade de um entrevistador. O conjunto de itens de Matemática, por exemplo, avalia a capacidade de raciocínio lógico e matemático dos indivíduos por meio de 12 itens que mesclam conhecimentos de Álgebra, Geometria e Trigonometria. Estas últimas são definidas como as dimensões formadoras do traço latente "raciocínio lógico e matemático"

Basicamente, os instrumentos são compostos por vários itens abertos ou fechados, com duas ou mais alternativas de resposta que podem ou não ser ordinais. Nos casos de itens fechados, que são os mais comuns, a cada alternativa de resposta, atribui-se um escore de forma que as categorias do item associadas a um maior nível do traço latente devem estar pontuadas no mesmo sentido. Tal pontuação pode estar no sentido positivo (quanto maior o nível do traço latente, maior o escore) ou negativo (quanto maior o nível do traço latente, menor o escore), bastando que seja o mesmo para todos os itens. Por exemplo, para avaliar o nível de depressão de um indivíduo através do sentimento de tristeza que eventualmente ele possa sentir, o item 1 do BDI (Apêndice B), apresenta 4 alternativas de resposta: "Não me sinto triste", "Eu me sinto triste", "Estou sempre triste e não consigo sair disso"e "Estou tão triste ou infeliz que não consigo suportar". As pontuações atribuídas a essas 4 categorias são $0,1,2$ e 3 , respectivamente. Ou seja, quanto mais triste o indivíduo se sente, maior o escore da categoria. Os demais itens do instrumento seguem o mesmo padrão de pontuação e, conseqüentemente, quanto maior o nível de depressão do indivíduo, maior será a pontuação em cada item, gerando um alto escore total no teste. O escore total atribuído a um indivíduo corresponde à soma dos escores das categorias dos itens por ele selecionadas e reflete sua condição com relação ao traço latente em questão 1 . No

\footnotetext{
${ }^{1}$ Para situações em que o instrumento avalia mais do que uma dimensão, além do escore total, podem-se
} 
vestibular, o escore 1 é atribuído à categoria correta do item e o escore 0 , às demais. Assim, os alunos aprovados são aqueles que acertaram um maior número de itens, ou seja, que têm maior escore total na prova. Um outro tipo de instrumento bastante interessante é o Teste de Investigação Bulímica de Edinburgh (TIBE), que também se encontra anexado. Ele se caracteriza pela mistura de itens abertos (1 a 5) e fechados (os demais), com diferentes números de categorias de respostas. Além disso, a amplitude de variação dos escores difere entre os itens, embora não existam razões clínicas para se acreditar que um item com escore máximo menor seja menos importante do que outro com escore máximo maior. Entretanto, quando o escore total é obtido, esta diferenciação de importância dos itens fica implícita, ocasionando um viés na quantificação do traço latente em questão.

Uma explicação mais detalhada sobre os princípios de elaboração desses instrumentos pode ser encontrada em Pedhazur \& Schmelkin (1991) e, mais especificamente na área de Psiquiatria, em Gorenstein, Andrade \& Zuardi (1999).

\subsection{Métodos Clássicos para o Estudo da Qualidade de Instrumentos de Avaliação Psiquiátrica}

Para evidenciar a boa ou má qualidade e a aplicabilidade de algum instrumento de avaliação são necessários vários estudos que visam avaliar a confiabilidade, a validade, a consistência interna e a dimensionalidade do instrumento. Os parágrafos abaixo apresentam uma breve definição dessas características e os métodos estatísticos classicamente adotados para seu estudo. Maiores explicações podem ser encontradas em Carmines \& Zeller (1979) ou em Pedhazur \& Schmelkin (1991).

A confiabilidade de um instrumento diz respeito à sua reprodutibilidade, ou seja, à concordância dos resultados obtidos em duas ou mais aplicações nos mesmos sujeitos (confiabilidade teste-reteste ou confiabilidade entre entrevistadores). Coeficientes de confiabilidade, como Kappa, Kappa ponderado ou coeficiente de correlação intraclasse, escolhidos de acordo com a natureza da medida gerada a partir do instrumento são muito utilizados na literatura.

A validade de um instrumento está relacionada com sua capacidade de medir o construto de interesse. Com o objetivo de avaliá-la, inicialmente, faz-se um estudo qualitativo buscando identificar se todos os aspectos pertencentes ao construto (e nenhum outro mais) estão contemplados nos itens do instrumento. Nesta etapa, a experiência clínica do pesquisador e seu conhecimento sobre as características do construto em questão é fundamental. De forma geral, pode-se dizer que quanto maior a capacidade do instrumento de avaliação para identificar e diferenciar os níveis do traço latente dos indivíduos, maior é

calcular sub-escores que consistem na soma dos escores atribuídos a alguns subconjuntos de itens. 
a sua validade. Em alguns casos em que há um padrão-ouro, ou seja, uma outra forma de se avaliar o construto de maneira confiável, podem-se calcular índices como sensibilidade, especificidade, valores preditivos positivo e negativo ou mesmo ajustar um modelo de análise discriminante ou regressão logística como critérios de verificação da qualidade do instrumento.

A consistência interna é uma característica do instrumento relacionada à homogeneidade e à clareza dos itens que o compõem. Itens ambíguos ou fora do contexto do que se deseja quantificar contribuem para a falta de consistência interna de um instrumento que, usualmente, é identificada pelo coeficiente alfa de Cronbach.

Por último, o número de dimensões, caracteriza-se pela quantidade de aspectos, representantes de um mesmo traço latente, contemplados no instrumento. Por exemplo, Wang et al. (2005) identificam duas dimensões, ambas associadas à depressão, presentes no BDI: aspectos cognitivo-afetivos e somáticos. A técnica estatística aplicada neste contexto costuma ser a análise fatorial.

\subsection{Vantagens da TRI para o Estudo da Qualidade de Instrumentos de Avaliação Psiquiátrica}

Principalmente pelo fato de a TRI ter sido originalmente aplicada em Educação e por não ter sido implementada ainda nos grandes pacotes estatísticos, é uma metodologia pouco utilizada na Psiquiatria, especialmente no Brasil. Por outro lado, a sua aplicação a testes de personalidade, atitudes comportamentais e diagnósticos tem crescido muito nos últimos anos (Embretson \& Reise 2000).

Resultados provenientes de aplicações de modelos da TRI não devem ser vistos apenas como substitutos dos resultados das análises psicométricas usuais, mencionadas na seção 1.4. Eles representam o estudo das propriedades estatísticas de um instrumento sob

um enfoque diferente. É claro que certa analogia entre os resultados da teoria clássica e da TRI é cabível e discutida na seção 2.4. Em particular, convém notar que os modelos utilizados na TRI permitem:

- Variar o erro padrão do traço latente segundo sua magnitude;

- Considerar medidas separadas, porém na mesma escala de medida, para a caracterização de indivíduos e de itens;

- Estimar o traço latente de um indivíduo com base em apenas alguns itens, sem a necessidade da aplicação de todo o instrumento. Esta característica é especialmente importante nos casos de instrumentos com um número muito grande de itens que, 
conjuntamente, devem avaliar diversos níveis do traço latente. A caracterização do indivíduo pode ser baseada apenas nas respostas àqueles itens adequados à avaliação do seu respectivo nível do traço latente. Esta é a base da construção de testes adaptativos computadorizados (para maiores explicações, vide Weiss (1985) e Ware, Bjomer \& Kosinski (2000));

- Padronizar as escalas de estimação do traço latente e dos parâmetros de dificuldade dos itens. Desta forma, quando algumas modificações no instrumento são necessárias, os traços latentes estimados para indivíduos que reponderam a versões diferentes tornam-se comparáveis;

- Estimar parâmetros de itens com números de categorias diferentes (com escores diferentes) sem provocar um desbalanceamento na estimação do traço latente.

Embretson \& Reise (2000) apresentam um estudo de simulação bastante detalhado de algumas outras vantagens da TRI sobre os métodos clássicos da psicometria. 


\section{Capítulo 2}

\section{Metodologia da TRI}

Na seção 2.1 são descritos os modelos da TRI mais comuns na área de Educação. Na seção 2.2 são apresentados métodos de estimação dos parâmetros de alguns dos modelos presentes na seção anterior. Na seção 2.3 são discutidos aspectos computacionais. Na seção 2.4. discute-se a aplicação de um dos modelos da TRI à área de Psiquiatria através de um conjunto de dados (Wang et al. 2005).

\subsection{Principais Modelos da TRI}

Os 4 primeiros modelos apresentados são aplicáveis a itens com respostas binárias e instrumentos compostos por apenas um traço latente (denominados unidimensionais). Mesmo que os itens sejam de múltipla escolha, com 4 ou 5 categorias por exemplo, a resposta dada a cada um pode ser dicotomizada com categorias correta, se o indivíduo respondeu corretamente ao item, ou incorreta, em caso contrário.

\section{Modelo logístico unidimensional de três parâmetros (ML3)}

O modelo é definido por

$$
P\left(X_{i j}=1 \mid \theta_{j}, \boldsymbol{\zeta}_{i}\right)=c_{i}+\left(1-c_{i}\right) \frac{1}{1+e^{-D a_{i}\left(\theta_{j}-b_{i}\right)}}
$$

com: (i) $\mathrm{i}=1,2, \ldots$, I indexando os itens do instrumento, (ii) $\mathrm{j}=1,2, \ldots, \mathrm{n}$ indexando os indivíduos que responderam aos itens, (iii) $X_{i j}$ representando uma variável dicotômica que assume o valor 1 quando o indivíduo j responde corretamente o item i e 0 em caso contrário, (iv) $\theta_{j}$ denotando o nível do traço latente do jésimo indivíduo, ou seja, a habilidade (ou proficiência) do indivíduo na disciplina 


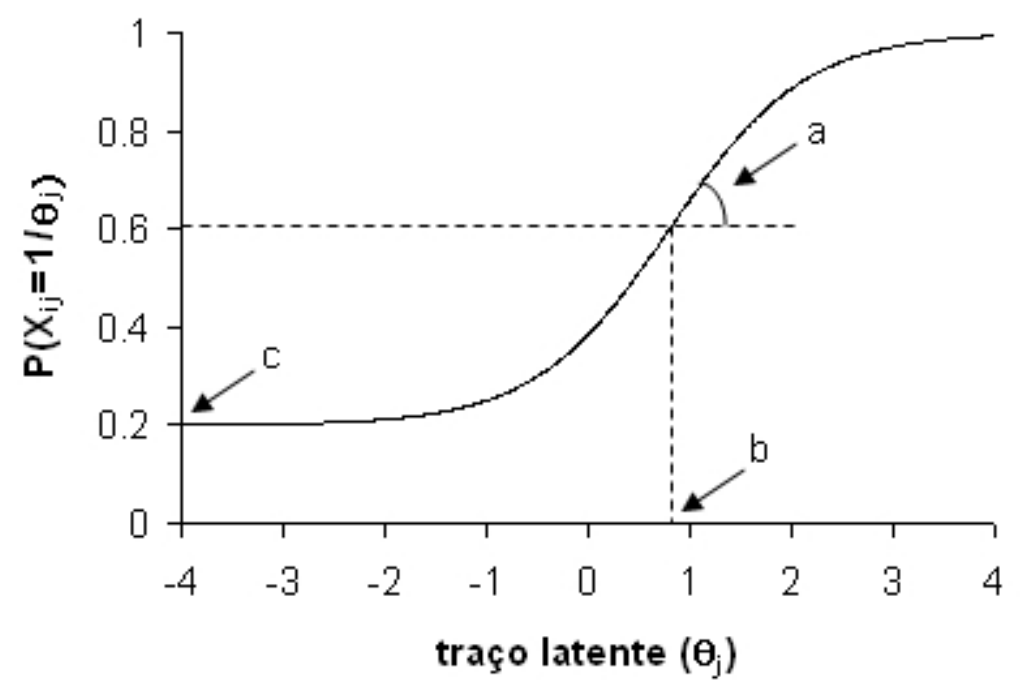

Figura 2.1: Exemplo de curva característica de um item sob o ML3

em questão, (v) $b_{i}$ indicando o parâmetro de localização ou de dificuldade do item i (medido na mesma escala do traço latente) que representa o nível latente em que a probabilidade de $X_{i j}=1$ é $\left(1+c_{i}\right) / 2$, (vi) $a_{i}$, parâmetro de discriminação ou de sensibilidade do item $i$, representando a inclinação da função no ponto em que o traço latente é igual a $b_{i}$, (vii) $c_{i}$ denotando o parâmetro do item que representa a probabilidade de indivíduos com baixo nível do traço latente $\left(\theta_{j} \rightarrow-\infty\right)$ responderem corretamente ao item i, (viii) $\boldsymbol{\zeta}_{i}=\left(a_{i} b_{i} c_{i}\right)^{t}$ e (ix) D indicando um fator de escala que pode assumir os valores 1 ou 1,7. Quando $\mathrm{D}$ é igual a 1,7, a função logística fornece resultados semelhantes ao da função ogiva normal (Hambleton, Swaminathan \& Rogers 1991).

Uma representação gráfica de (2.1) é a curva característica do item (CCI), que descreve a relação entre a probabilidade de acerto do item e o traço latente (Figura 2.1). Pela CCI, pode-se perceber que a probabilidade de acerto do item i, $P\left(X_{i j}=1 \mid\right.$ $\left.\theta_{j}, \boldsymbol{\zeta}_{i}\right)$, cresce com o aumento do nível do traço latente $\theta_{j}$. Note, também, que para traços latentes muito grandes $\left(\theta_{j} \rightarrow \infty\right), P\left(X_{i j}=1 \mid \theta_{j}, \boldsymbol{\zeta}_{i}\right)=1$.

A intensidade de crescimento dessas probabilidades foi base para a definição de um critério muito utilizado na interpretação dos resultados do modelo, isto é, a definição de itens âncora.

Um item é definido como âncora num determinado nível latente $\theta_{j}$ se:

$$
\begin{gathered}
P\left(X_{i j}=1 \mid \theta_{j}-\delta, \boldsymbol{\zeta}_{i}\right) \leq 0,50, \\
P\left(X_{i j}=1 \mid \theta_{j}, \boldsymbol{\zeta}_{i}\right) \geq 0,65 \mathrm{e}
\end{gathered}
$$




$$
P\left(X_{i j}=1 \mid \theta_{j}, \boldsymbol{\zeta}_{i}\right)-P\left(X_{i j}=1 \mid \theta_{j}-\delta, \boldsymbol{\zeta}_{i}\right) \geq 0,30,
$$

em que $\delta$ é fixado de acordo com a escala de medida (ou métrica) do parâmetro $\theta_{j} 11$. Por exemplo, quando definem-se média e desvio padrão de $\theta_{j}$ iguais a 0 e 1 , respectivamente, é comum estipular-se $\delta=1$ para um intervalo de variação [-3,3] do traço latente. Assim, verifica-se se cada item do instrumento pode ser considerado como âncora nos níveis dos traços latentes latentes definidos em -2, -1, 0, 1, 2 e 3 . Mais especificamente, para um item ser âncora em um determinado nível $\theta$ do traço latente, ele deve ser acertado por pelo menos $65 \%$ dos indivíduos com traço latente igual a $\theta$ e por menos de $50 \%$ dos indivíduos com traço latente no nível imediatamente anterior $(\theta-\delta)$. Adicionalmente, a diferença entre as proporções de indivíduos que acertam tal item nesses dois níveis latentes $(\theta$ e $\theta-\delta)$ deve ser de, no mínimo, 30\%. Em outras palavras, um item âncora no nível do traço latente $\theta$ é um item com alta probabilidade de acerto por indivíduos com tal nível, mas pouco acertado por indivíduos com níveis menores. Tal definição pode ser adotada para todos os modelos da TRI para dados dicotômicos.

\section{Modelo logístico unidimensional de dois parâmetros (ML2)}

Este modelo equivale a (2.1) $\operatorname{com} c_{i}=0$ para $\mathrm{i}=1,2, \ldots$, I e é dado por

$$
P\left(X_{i j}=1 \mid \theta_{j}, \boldsymbol{\zeta}_{i}\right)=\frac{1}{1+e^{-D a_{i}\left(\theta_{j}-b_{i}\right)}} .
$$

Uma representação gráfica das CCI de 2 itens que seguem o modelo $(2.2)$ é dada na Figura 2.2.

\section{Modelo logístico unidimensional de um parâmetro (ML1) - Modelo de Rasch (1960)}

Esse modelo equivale a 2.1 com $a_{i}=a=1$ e $c_{i}=0$ para $\mathrm{i}=1,2, \ldots, \mathrm{I}$ e é dado por

$$
P\left(X_{i j}=1 \mid \theta_{j}, \boldsymbol{\zeta}_{i}\right)=\frac{1}{1+e^{-D a\left(\theta_{j}-b_{i}\right)}} .
$$

A Figura 2.3 representa a CCI de dois itens que seguem um modelo de Rasch (1960). A característica que diferencia esse modelo dos outros anteriormente apresentados em (2.1) e 2.2 é o fato de o parâmetro de discriminação $\left(a_{i}\right)$ ser comum a todos os itens, ou seja, a inclinação das respectivas curvas é a mesma e elas não se encontram (a não ser em $\infty$ e $-\infty)$. Isto significa que se $P\left(X_{i j}=1 \mid \theta_{j}, \boldsymbol{\zeta}_{i}\right)>P\left(X_{i^{\prime} j}=1 \mid \theta_{j}, \boldsymbol{\zeta}_{i^{\prime}}\right)$, então $P\left(X_{i j^{\prime}}=1 \mid \theta_{j^{\prime}}, \boldsymbol{\zeta}_{i}\right)>P\left(X_{i^{\prime} j^{\prime}}=1 \mid \theta_{j^{\prime}}, \boldsymbol{\zeta}_{i^{\prime}}\right)$, para $i \neq i^{\prime}$ e $j \neq j^{\prime}$. Ou seja, se a probabilidade de acerto de um item i é maior do que a de um outro item $i^{\prime}$ para

\footnotetext{
${ }^{1}$ Para resolver um problema de falta de identificabilidade do modelo, a escala de medida de $\theta_{j}$ é estabelecida fixando-se os respectivos valores de média e desvio-padrão. Uma explicação mais detalhada sobre a definição da métrica de $\theta_{j}$ será apresentada na seção 2.2
} 


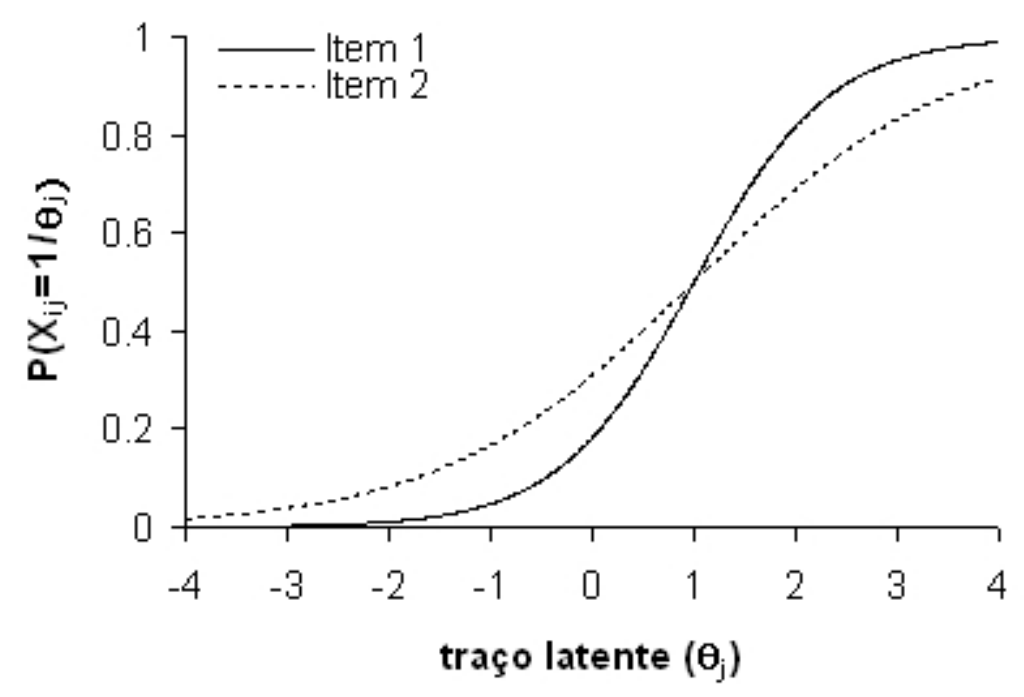

Figura 2.2: CCI de dois itens sob o ML2

um determinado nível do traço latente $\theta_{j}$, ela permanecerá sendo maior em qualquer outro nível do traço latente $\theta_{j^{\prime}}$.

\section{Modelo logístico unidimensional de quatro parâmetros (ML4)}

O modelo é dado por

$$
P\left(X_{i j}=1 \mid \theta_{j}, \boldsymbol{\zeta}_{i}\right)=c_{i}+\left(\gamma_{i}-c_{i}\right) \frac{1}{1+e^{-D a_{i}\left(\theta_{j}-b_{i}\right)}}
$$

com $\boldsymbol{\zeta}_{i}=\left(a_{i} b_{i} c_{i} \gamma_{i}\right)^{t}$, os demais parâmetros definidos da mesma maneira que em (2.1) e $\gamma_{i}$ representando a probabilidade de indivíduos com alto nível do traço latente $\left(\theta_{j} \rightarrow \infty\right)$ responderem corretamente ao item i (probabilidade de descuido). Note que, no modelo (2.1), essa probabilidade é igual a $1 \mathrm{e}$, no modelo (2.4), $P\left(X_{i j}=\right.$ $\left.1 \mid \theta_{j}, \boldsymbol{\zeta}_{i}\right)=\gamma_{i} \leq 1$, quando $\left(\theta_{j} \rightarrow \infty\right)$. A CCI de um item sob o modelo ML4 está representada na Figura 2.4 .

Vários modelos para itens com mais de uma categoria de resposta (nominais ou ordinais) têm sido propostos. Neste trabalho, apenas o modelo proposto por Samejima (1969) é citado. Outros modelos como o de escala gradual ((Andrich 1978a) e (Andrich 1978b)), de crédito parcial (Masters 1982) ou o de resposta nominal (Bock 1972) podem ser encontrados em Linden \& Hambleton (1996) e em Andrade, Tavares \& Valle (2000). 


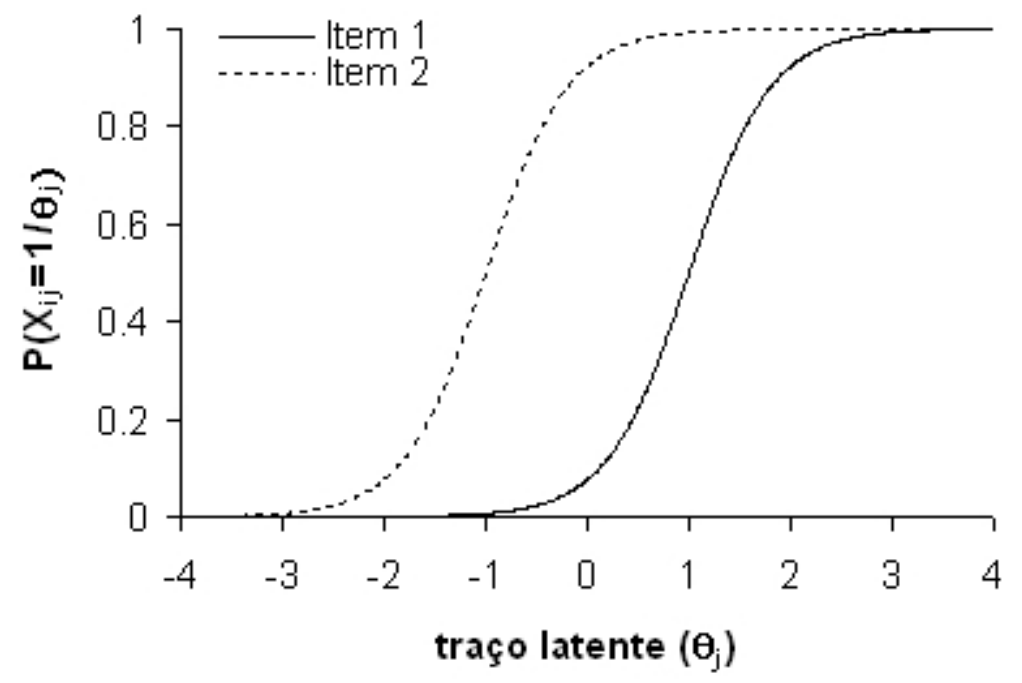

Figura 2.3: CCI de dois itens sob o ML1 - modelo de Rasch

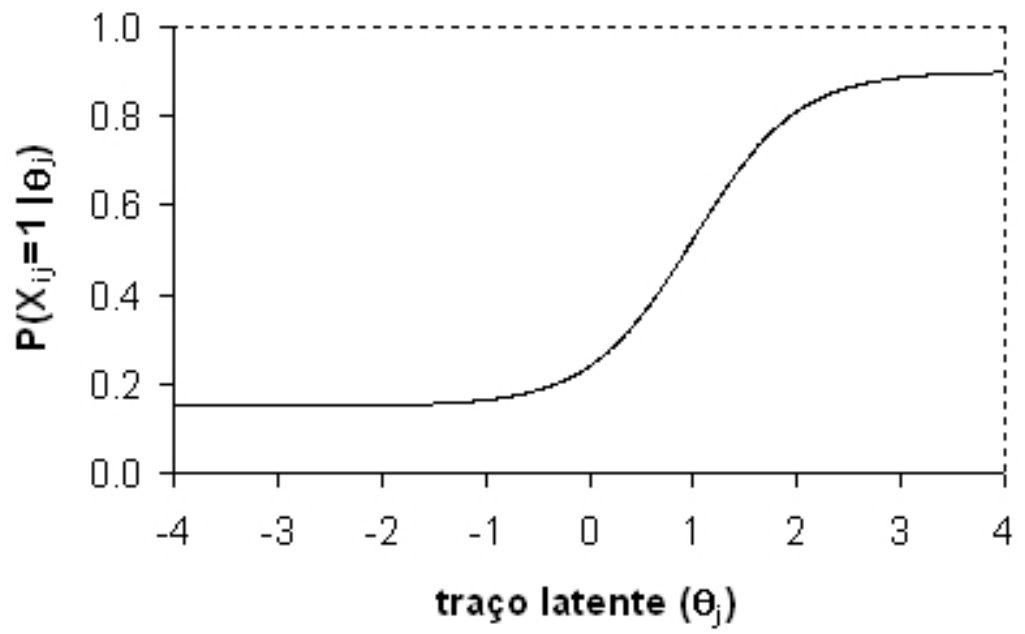

Figura 2.4: CCI de um item sob o ML4 


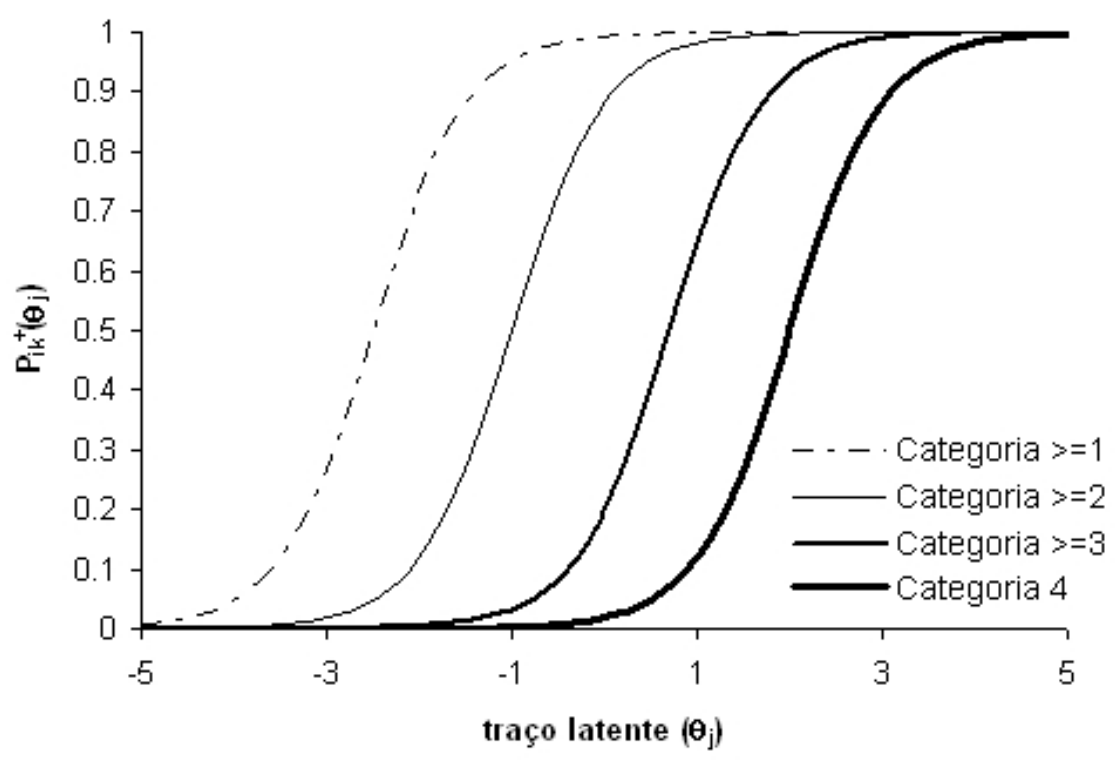

Figura 2.5: Probabilidade acumulada da escolha de cada categoria de um item sob o modelo de Samejima

\section{Modelo de resposta gradual para itens com respostas ordinais - Modelo de Samejima (1969)}

Este modelo é uma extensão de (2.2) para respostas ordinais. O número de categorias de resposta não necessita ser o mesmo para todos os itens do instrumento.

Cada item é caracterizado por um parâmetro de discriminação $\left(a_{i}\right)$ e $m_{i}$ parâmetros de localização. O número de categorias ordinais de resposta do i-ésimo item é $K_{i}=m_{i}+1$. A probabilidade de um indivíduo com nível do traço latente igual a $\theta_{j}$ escolher a categoria de resposta $\mathrm{k}$ ou qualquer outra de ordem acima de $\mathrm{k}$ no item i é

$$
P_{i k}^{+}\left(\theta_{j}\right)=\frac{1}{1+e^{-D a_{i}\left(\theta_{j}-b_{i k}\right)}}
$$

com: (i) $\mathrm{i}=1, \ldots, \mathrm{I}, \mathrm{j}=1, \ldots, \mathrm{n}, \mathrm{D}$ e $\theta_{j}$ definidos de maneira análoga a (2.1), (ii) $\mathrm{k}=0,1$, ..., $m_{i}$, (iii) $a_{i}$ denotando o parâmetro de discriminação comum a todas as categorias do item i e (iv) $b_{i k}$ indicando o parâmetro de localização que representa o nível latente necessário para a escolha da categoria de resposta $\mathrm{k}$ ou acima de $\mathrm{k}$ com probabilidade igual a 0.50 . Pela própria definição do modelo, temos que $b_{i 1} \leq b_{i 2} \leq \ldots \leq b_{i m_{i}}$. A Figura 2.5 representa as probabilidades acumuladas das escolhas das categorias de um item com 5 categorias.

Obviamente, a probabilidade de um indivíduo escolher a primeira categoria de resposta ou qualquer uma acima dela é $1\left(P_{i 0}^{+}\left(\theta_{j}\right)=1\right)$ e a probabilidade de um 


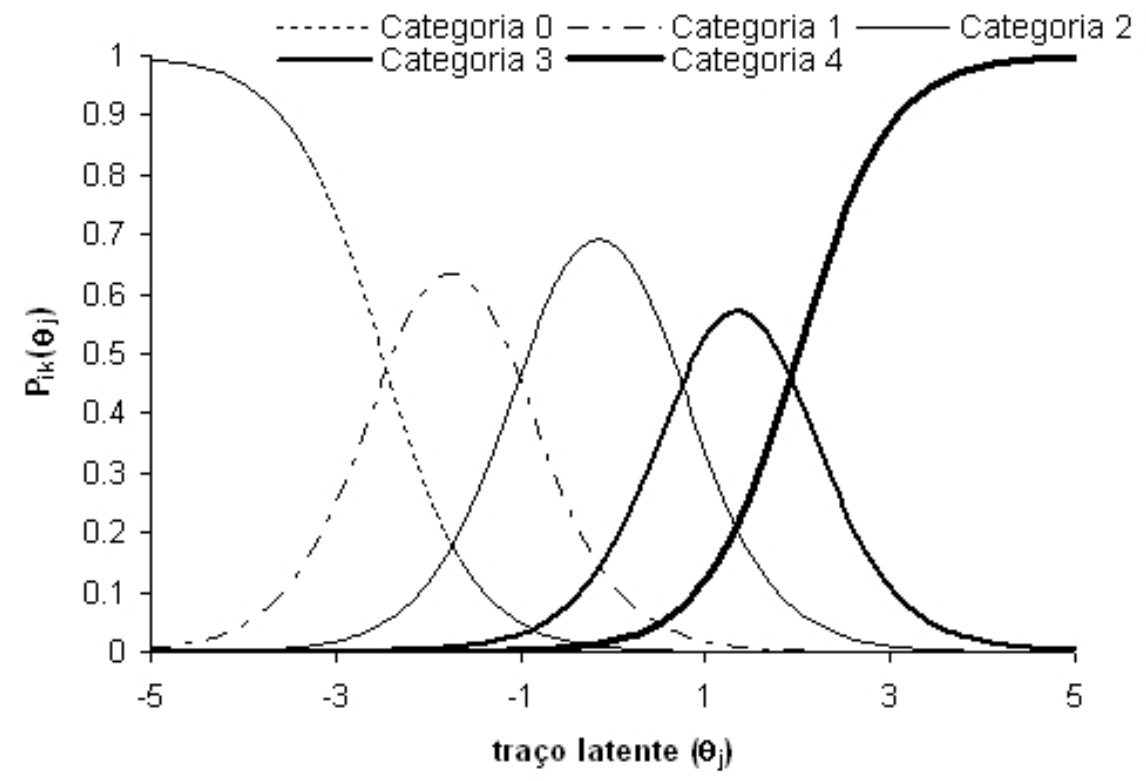

Figura 2.6: Probabilidade da escolha de cada categoria de um item sob o modelo de Samejima

indivíduo escolher qualquer categoria acima da última é $0\left(P_{i m_{i+1}}^{+}\left(\theta_{j}\right)=0\right)$. Desta forma, pode-se definir a probabilidade de um indivíduo com nível do traço latente igual a $\theta_{j}$ escolher a categoria de resposta k no item i por $P_{i k}\left(\theta_{j}\right)=P_{i k}^{+}\left(\theta_{j}\right)-P_{i k+1}^{+}\left(\theta_{j}\right)$. Reescrevendo essa equação com a notação de (2.5), o modelo de Samejima pode ser escrito como:

$$
P_{i k}\left(\theta_{j}\right)=\frac{1}{1+e^{-D a_{i}\left(\theta_{j}-b_{i k}\right)}}-\frac{1}{1+e^{-D a_{i}\left(\theta_{j}-b_{i k+1}\right)}} .
$$

A Figura 2.6 representa as probabilidades $P_{i k}\left(\theta_{j}\right)$ sob o modelo de Samejima.

A grande maioria das aplicações da TRI utiliza modelos unidimensionais. Apesar de alguns instrumentos de avalição envolverem mais de uma dimensão, é razoável supor que há uma dimensão dominante e que as demais podem ser ignoradas, sem comprometer significativamente o bom ajuste aos dados. Por exemplo, em uma avaliação da proficiência em Matemática, o aluno estará sendo avaliado quanto à proficiência em Álgebra, Geometria, Trigonometria e, até mesmo, Português, no mínimo para a compreensão de cada item. No entanto, as análises estatísticas são realizadas considerando-se uma única dimensão, que pode ser interpretada como a capacidade do raciocício lógico e exato do indivíduo. Neste contexto, é importante diferenciar um instrumento multidimensional do multivariado. A prova da primeira fase da Fuvest pode ser uma boa ilustração de instrumento de avaliação multivariado. Várias disciplinas compõem a prova (Matemática, Português, Biologia, etc), caracterizando 
a quantificação de mais de um construto, particularmente, um para cada disciplina.

Nem todos os casos podem ser reduzidos à unidimensionalidade. Algumas vezes, a qualidade de ajuste de um modelo unidimensional fica comprometida quando ele é aplicado a um instrumento multidimensional. Para situações desse tipo, alguns modelos unidimensionais da TRI já foram generalizados. Reckase (1997) ilustra alguns desses modelos para respostas dicotômicas, comentando sobre suas diferenças e perpectivas. Li \& Lissitz (2000) apresentam uma discussão a respeito da extensão multidimensional do modelo logístico de 3 parâmetros, descrito a seguir. Gardner, Kelleher \& Pajer (2000) descrevem uma generalização multidimensional do modelo de Samejima aplicado a um teste adaptativo computadorizado. Rost \& Carstensen (2002) descrevem o modelo multidimensional de Rasch e discutem sua relação com outros modelos já desenvolvidos.

\section{Modelo logístico multidimensional de três parâmetros}

O modelo é dado por

$$
P\left(X_{i j}=1 \mid \boldsymbol{\theta}_{j}\right)=c_{i}+\left(1-c_{i}\right) \frac{1}{1+e^{-\sum_{k=1}^{m} a_{i k} \theta_{j k}-d_{i}}}
$$

com: (i) i, j, $X_{i j}, c_{i}$, definidos como anteriormente, (ii) $\mathrm{k}=1, \ldots \mathrm{m}$, (iii) $\boldsymbol{\theta}_{j}=$ $\left(\begin{array}{llll}\theta_{j 1} & \theta_{j 2} & \ldots & \theta_{j m}\end{array}\right)$ denotando o vetor de dimensão $m$ dos traços latentes do j-ésimo indivíduo, (iv) $d_{i}$ representando o parâmetro de localização relacionado à dificuldade do item i e $(\mathrm{v}) a_{i k}$ indicando o parâmetro de discriminação do item i associado ao traço latente $\mathrm{k}$.

Esse modelo também é chamado de compensatório porque níveis baixos de alguns traços latentes podem ser compensados por níveis altos de outros traços latentes no

valor de $P\left(X_{i j}=1 \mid \boldsymbol{\theta}_{j}\right)$. Takane \& Leeuw (1988) discutem a semelhança entre a TRI multidimensional e a análise fatorial exploratória.

\subsection{Métodos de Estimação}

Os modelos da TRI envolvem a estimação de dois tipos de parâmetros: aqueles relacionados aos itens e aqueles associados ao traço latente individual. Em algumas situações, um dos tipos pode ser conhecido. Por exemplo, uma prova pode ser composta por alguns itens que já foram aplicados anteriormente. O parâmetros desses itens podem, portanto, ser considerados conhecidos com valores iguais às estimativas calculadas com as respostas obtidas no passado. Na área de Educação existem diversos "bancos de itens" com itens de parâmetros conhecidos (estimados anteriormente). Nesta seção, aborda-se o caso mais geral, em que ambos os tipos de parâmetros são desconhecidos e devem ser estimados. 
Esta é a situação mais comum na prática. Os estimadores serão descritos com base no modelo logístico unidimensional de 3 parâmetros para itens dicotômicos. Procedimentos de estimação para outros modelos e resultados mais detalhados sobre todos os procedimentos apresentados a seguir podem ser encontrados em Baker \& Kim (2004) e Andrade et al. (2000), por exemplo.

Basicamente são três os métodos utilizados para estimação: máxima verossimilhança conjunta, máxima verossimilhança marginal e métodos bayesianos.

Duas suposições são necessárias para os processos de estimação aqui focados: independência entre as respostas de diferentes indivíduos e independência entre as respostas dos itens pelo mesmo indivíduo, condicionalmente ao traço latente $\left(\theta_{j}\right)$. Esta última suposição significa que, dado o nível do traço latente do indívíduo, suas respostas a diferentes itens são independentes, ou seja, o indivíduo não adquire mais conhecimentos no decorrer do teste. A verossimilhança pode ser escrita como:

$$
\begin{aligned}
L(\boldsymbol{\theta}, \boldsymbol{\zeta}) & =\prod_{i=1}^{I} \prod_{j=1}^{n} P\left(X_{i j}=x_{i j} \mid \theta_{j}, \boldsymbol{\zeta}_{i}\right) \\
& =\prod_{i=1}^{I} \prod_{j=1}^{n} P\left(X_{i j}=1 \mid \theta_{j}, \boldsymbol{\zeta}_{i}\right)^{x_{i j}} P\left(X_{i j}=0 \mid \theta_{j}, \boldsymbol{\zeta}_{i}\right)^{\left(1-x_{i j}\right)}
\end{aligned}
$$

com: (i) i, j, $X_{i j}, \theta_{j}$ definidos anteriormente, (ii) $\boldsymbol{\theta}=\left(\begin{array}{llll}\theta_{1} & \theta_{2} & \ldots & \theta_{n}\end{array}\right)^{t}$ denotando o vetor (nx1) dos traços latentes dos $n$ indivíduos e (iii) $\boldsymbol{\zeta}=\left(\begin{array}{llll}\boldsymbol{\zeta}_{1} & \boldsymbol{\zeta}_{2} & \ldots & \boldsymbol{\zeta}_{I}\end{array}\right)^{t}$, em que $\boldsymbol{\zeta}_{i}=\left(\begin{array}{lll}a_{i} & b_{i} & c_{i}\end{array}\right)^{t}$ indica o vetor dos parâmetros do item i representando o vetor (3Ix1) dos parâmetros dos itens.

O logaritmo da verossimilhança é

$$
\log L(\boldsymbol{\theta}, \boldsymbol{\zeta})=\sum_{i=1}^{I} \sum_{j=1}^{n} x_{i j} \log P\left(X_{i j}=1 \mid \theta_{j}, \boldsymbol{\zeta}_{i}\right)+\left(1-x_{i j}\right) \log P\left(X_{i j}=0 \mid \theta_{j}, \boldsymbol{\zeta}_{i}\right)
$$

Uma restrição necessária para a estimação diz respeito à métrica de estimação dos parâmetros $a_{i}, b_{i}$ e $\theta_{j}$, pois os modelos ML2, ML3 e ML4 são não-identificáveis, ou seja, mais de uma combinação de valores desses parâmetros levam ao mesmo valor de $P\left(X_{i j}=\right.$ $\left.1 \mid \theta_{j}, \boldsymbol{\zeta}_{i}\right)$. A solução mais usada para eliminar tal problema de identificabilidade é fixar a média e o desvio-padrão de $\theta_{j}$ (normalmente, mas não necessariamente, em 0 e 1 ). Quando a média é fixada em 0 e o desvio-padrão em 1 (e quando $\mathrm{D}=1$, no modelo (2.1)), na prática, esperam-se valores de $a_{i}$ entre 0 e 2 , sendo que os mais apropriados seriam aqueles maiores do que 1 , e valores entre -2 e 2, para os parâmetros $b_{i}$ (Andrade et al. 2000). 


\section{Método de Máxima Verossimilhança Conjunta}

Maximizando a função log-verossimilhança (2.9) com relação a cada um dos parâmetros $a_{i}, b_{i}, c_{i}$ e $\theta_{j}$, obtêm-se as equações de estimação e, resolvendo-as, obtêm-se as estimativas de máxima verossimilhança. A maximização não pode ser resolvida analiticamente e a utilização de métodos iterativos faz-se necessária. O procedimento de estimação costuma ser dividido em dois devido ao grande número de parâmetros a serem estimados simultaneamente $(3 \mathrm{I}+\mathrm{n})$ : no primeiro estágio, os parâmetros dos itens são estimados assumindo-se que os traços latentes dos indivíduos são conhecidos; no segundo estágio, os traços latentes são estimados, supondo-se conhecidos os parâmetros dos itens.

Um dos problemas desse método é a indefinição de estimadores para indivíduos que apresentam respostas iguais a 1 (ou 0 ) para todos os itens ou para itens que apresentam respostas 1 (ou 0) para todos os indivíduos. Além disso, as suposições necessárias para obtenção das propriedades assintóticas dos estimadores de máxima verossimilhança não são satisfeitas; os estimadores resultantes são inconsistentes. Por não impor nenhuma restrição no espaço paramétrico, as estimativas de alguns dos parâmetros de item podem estar fora dos intervalos adequados: $a_{i} \geq 0$ e $0 \leq c_{i} \leq 1$.

\section{Método de Máxima Verossimilhança Marginal}

O método de máxima verossimilhança marginal também é aplicado dividindo-se o procedimento de estimação em dois estágios. Numa primeira etapa, estimam-se os parâmetros dos itens através da marginalização da função de verossimilhança em relação a $\theta_{j}$. Numa segunda etapa, considerando-se os parâmetros de itens conhecidos e iguais aos obtidos na etapa 1 , encontram-se os estimadores dos traços latentes individuais, $\theta_{j}$.

Para a estimação dos parâmetros de itens, assume-se que os indivíduos são selecionados aleatoriamente de uma população cujos traços latentes, $\theta_{j}$, seguem uma determinada distribuição $g(\theta \mid \tau)$. Neste caso, pelo valor fixado para $\tau$, a métrica de estimação dos parâmetros já está definida. Comumente, $g(\theta \mid \tau)$ é escolhida como uma distribuição $\operatorname{Normal}(0,1)$. Integrando-se a verossimilhança $(2.8)$ em relação a $\theta$, tem-se (pela independência entre as respostas de diferentes indivíduos e pela independência das respostas aos itens condicionalmente a $\theta_{j}$ ):

$$
L(\boldsymbol{\tau}, \boldsymbol{\zeta} \mid \boldsymbol{X})=\prod_{j=1}^{n} \int_{\mathbb{R}} \prod_{i=1}^{I} P\left(X_{i j}=1 \mid \theta, \boldsymbol{\zeta}_{i}\right)^{x_{i j}} P\left(X_{i j}=0 \mid \theta, \boldsymbol{\zeta}_{i}\right)^{\left(1-x_{i j}\right)} g(\theta \mid \boldsymbol{\tau}) d \theta
$$

Maximiza-se 2.10), em relação a $\boldsymbol{\zeta}_{i}=\left(\begin{array}{lll}a_{i} & b_{i} & c_{i}\end{array}\right)$, e, através de algum processo iterativo, encontram-se as estimativas dos parâmetros de item. As habilidades são estimadas, então, considerando-se os parâmetros de item conhecidos e com valores iguais aos encontrados no passo anterior. 
Os problemas de indefinição de estimadores para indivíduos ou itens que apresentam respostas todas iguais e estimativas de $a_{i}$ e $c_{i}$ fora dos intervalos esperados, citados no método de máxima verossimilhança conjunta, permanecem. Porém, os estimadores de máxima verossimilhança marginal são consistentes.

\section{Método Bayesiano Marginal}

O método de estimação bayesiano envolve a atribuição de distribuições a priori para os parâmetros dos itens e para os traços latentes dos indivíduos. Pelo Teorema de Bayes, escontra-se a distribuição a posteriori para o conjunto de todos os parâmetros de interesse, condicionalmente aos valores observados. As respectivas estimativas podem ser obtidas com base em alguma característica dessa distribuição, tal como a esperança ou a moda. A distribuição a posteriori para o conjunto de todos os parâmetros de interesse pode ser escrita como:

$$
f(\boldsymbol{\theta}, \boldsymbol{\zeta}, \boldsymbol{\tau}, \boldsymbol{\eta} \mid \boldsymbol{X}) \propto L(\boldsymbol{\theta}, \boldsymbol{\zeta} \mid \boldsymbol{X}) g(\boldsymbol{\theta} \mid \boldsymbol{\tau}) f(\boldsymbol{\zeta} \mid \boldsymbol{\eta}) g(\boldsymbol{\tau}) f(\boldsymbol{\eta})
$$

com (i) $\boldsymbol{X}=\left(\boldsymbol{X}_{\cdot 1}, \ldots, \boldsymbol{X}_{\cdot n}\right)^{t}$, em que $\boldsymbol{X}_{\cdot j}=\left(X_{1 j}, \ldots, X_{I j}\right)$ denotando a matriz das respostas e (ii) $g(\boldsymbol{\theta} \mid \boldsymbol{\tau})$ e $f(\boldsymbol{\zeta} \mid \boldsymbol{\eta})$ representando as distribuições a priori dos traços latentes e dos parâmetros dos itens, respectivamente. As distribuições a priori para os parâmetros $\boldsymbol{\tau}$ e $\boldsymbol{\eta}$ são denotadas por $g(\boldsymbol{\tau})$ e $f(\boldsymbol{\eta})$. A métrica dos estimadores é baseada na escolha dessas distribuições a priori.

Integrando (2.11) com relação a $\boldsymbol{\theta}$ e $\boldsymbol{\eta}$, obtém-se a distribuição marginal a posteriori $f(\boldsymbol{\zeta}, \boldsymbol{\tau} \mid \boldsymbol{X})$ dada por:

$$
\begin{aligned}
f(\boldsymbol{\zeta}, \boldsymbol{\tau} \mid \boldsymbol{X}) & =C \iint L(\boldsymbol{\theta}, \boldsymbol{\zeta}) g(\boldsymbol{\theta} \mid \boldsymbol{\tau}) f(\boldsymbol{\zeta} \mid \boldsymbol{\eta}) g(\boldsymbol{\tau}) f(\boldsymbol{\eta}) d \boldsymbol{\theta} d \boldsymbol{\eta} \\
& =C g(\boldsymbol{\tau}) \int f(\boldsymbol{\zeta} \mid \boldsymbol{\eta}) f(\boldsymbol{\eta}) d \boldsymbol{\eta} \int L(\boldsymbol{\theta}, \zeta) g(\boldsymbol{\theta} \mid \boldsymbol{\tau}) d \boldsymbol{\theta} \\
& \propto L(\boldsymbol{\zeta}, \boldsymbol{\tau}) f(\boldsymbol{\zeta}) g(\boldsymbol{\tau})
\end{aligned}
$$

Maximizando 2.12 em relação a $\boldsymbol{\zeta}$, através de algum processo iterativo, encontramse os estimadores bayesianos (a moda a posteriori) dos parâmetros dos itens. Note que, apesar de 2.12 não depender de $\boldsymbol{\theta}$, ela depende da distribuição a priori $g(\boldsymbol{\tau})$, o que garante a definição da métrica de estimação dos parâmetros dos itens. As habilidades são estimadas, então, considerando-se os parâmetros de item conhecidos e iguais aos encontrados no passo anterior. Neste contexo, uma estimação bayesiana também pode ser obtida encontrando-se a distribuição a posteriori dos traços latentes supondo-se conhecidos os parâmetros dos itens. Os estimadores bayesianos correspondem à moda a posteriori (MAP) ou à esperança a posteriori (EAP), por exemplo.

Duas vantagens do método bayesiano são: as estimativas para os parâmetros dos itens estão definidas em todas as situações e, escolhendo distribuições a priori adequadas, as estimativas não extrapolam os limites do espaço paramétrico. 
Como os valores devem ser positivos, para $a_{i}$, estar entre zero e um, para $c_{i}$, e podem ser quaisquer valores reais, para $b_{i}$ e $\theta_{j}$, as distribuições a priori comumente escolhidas são, respectivamente, Log-normal, Beta, Normal e Normal.

\section{Monte Carlo via Cadeia de Markov (MCMC)}

A idéia geral do método MCMC é simular uma cadeia de Markov cuja distribuição estacionária é a distribuição de interesse, neste caso, a distribuição a posteriori dos parâmetros $\boldsymbol{\theta}$ e $\boldsymbol{\zeta}$ condicionalmente aos valores observados. Patz \& Junker (1999) descrevem o método MCMC especificamente para obtenção das estimativas dos parâmetros do ML3 da TRI.

\subsection{Aspectos Computacionais}

Vários programas podem ser utilizados para ajustar modelos da TRI. Entre eles, podem ser citados:

- Bilog e Bilog-MG

Ajustam os modelos unidimensionais logísticos e da função ogiva Normal (modelos logísticos considerando-se $\mathrm{D}=1,7)$ com 1,2 e 3 parâmetros para dados dicotômicos. Os métodos de estimação de máxima verossimilhança marginal e bayesiano marginal podem ser adotados para estimação dos parâmetros dos itens e os métodos bayesianos com estimação pela média a posteriori, pela moda a posteriori ou o de máxima verossimilhança podem ser adotados para os traços latentes. O Bilog permite o ajuste de modelos da TRI apenas para um grupo de indivíduos, enquanto que o Bilog-MG permite a definição de vários grupos de indivíduos com diferentes distribuições do traço latente.

- $\underline{\text { Xcalibre }}$

Apresenta as mesmas opções de ajuste que o Bilog, à exceção do modelo de 1 parâmetro.

- Multilog

Ajusta modelos unidimensionais da TRI para dados dicotômicos ou politômicos. Os modelos considerados são o logístico de 1, 2 e 3 parâmetros, de resposta gradual de Samejima, de crédito parcial (Masters 1982) e o de resposta nominal (Bock 1972). O método de estimação de máxima verossimilhança marginal é adotado para estimação dos parâmetros dos itens e os métodos bayesiano com estimação máximo a posteriori ou de máxima verossimilhança podem ser adotados para os traços latentes. Também permite o ajuste para múltiplos grupos. 


\section{- $\underline{\text { Parscale }}$}

Ajusta modelos unidimensionais da TRI para dados dicotômicos ou politômicos. Os modelos considerados são o logístico de 1,2 e 3 parâmetros, de resposta gradual de Samejima, de escala gradual (que nada mais é do que um caso particular do modelo de resposta gradual de Samejima, proposto por Andrich (1978b), de crédito parcial (Masters 1982) e de crédito parcial generalizado (Muraki 1992). O método de estimação de máxima verossimilhança marginal é adotado para estimação dos parâmetros dos itens e os métodos bayesiano com estimação pela média a posteriori ou de máxima verossimilhança podem ser adotados para os traços latentes. Também permite o ajuste para múltiplos grupos.

- $\underline{\text { Noharm }}$

Ajusta modelos uni e multidimensionais da TRI que seguem a função ogiva Normal. O software e seu manual podem ser obtidos gratuitamente em

http://www.unt.edu/rss/class/rich/5840/mcdonald/hoharm/NOHARM\%20Downloads.htm

\subsection{Aplicação à Psiquiatria}

Um dos modelos mais adotados nessa área é o de Rasch (1960) para itens com escala dicotômica e unidimensional (ML1), provavelmente devido à sua simplicidade. É um modelo intuitivamente adequado apenas para alguns instrumentos psiquiátricos e, na prática, costuma não ser o que melhor se adapta aos dados (Kessler, Andrews, Colpe, Hiripi, Mroczek, Normand, Walters \& Zaslavsky 2002). A existência de uma grande variedade de instrumentos psiquiátricos com peculiaridades intrínsecas deve ser levada em consideração na escolha do modelo mais adequado. Fatores como o número de categorias dos itens, a uni ou multidimensionalidade do instrumento, a capacidade de discriminação dos itens e, principalmente, a interpretação clínica de todos os parâmetros do modelo escolhido são os principais.

Nesta seção, os parâmetros do modelo logístico unidimensional de 3 parâmetros serão interpretados no contexto do BDI. Os parâmetros dos demais modelos apresentados na seção 2.1 podem ser facilmente interpretados por analogia. Redefinindo os parâmetros, índices e variáveis da equação (2.1), tem-se: (i) $\mathrm{i}=1,2, \ldots$. I indexando os itens do instrumento (no caso do BDI, I=21), (ii) $\mathrm{j}=1,2, \ldots, \mathrm{n}$ indivíduos que responderam aos itens ( $\mathrm{n}=208$ imigrantes chineses residentes em São Paulo), (iii) $X_{i j}$ representando uma variável dicotômica que assume o valor 1 , quando o indivíduo j relata apresentar o sintoma descrito no item i (ou seja, escolhe uma das categorias com escores 1, 2 ou 3 do respectivo item) e 0 em caso contrário, (iv) $\theta_{j}$ denotando o nível do traço latente do j-ésimo indivíduo, ou seja, sua respectiva intensidade (ou gravidade) de depressão, (v) $b_{i}$ indicando o parâmetro de gravidade de depressão avaliada pelo item i, medido na mesma 
escala do traço latente ${ }^{2}$, (vi) $a_{i}$, parâmetro de discriminação ou de sensibilidade do item i, representando a inclinação da função no ponto em que a intensidade de depressão é igual a $b_{i}$, (vii) $c_{i}$ denotando a probabilidade de indivíduos com baixa intensidade de depressão apresentarem o sintoma descrito no item i e (viii) D indicando um fator de escala que pode assumir os valores 1 ou 1,7.

Ainda se discute a interpretação e a necessidade de inclusão do parâmetro $c_{i}$ no modelo. Hays, Morales \& Reise (2000), interpretam-no como uma probabilidade de erro de resposta ao item, no sentido de que um indivíduo que não tem um nível de traço latente (intensidade de depressão) grande o suficiente para apresentar o sintoma mas, incoerentemente, refere sua presença. Essa explicação pode ser relacionada ao conceito de falso-positivo: o indivíduo não tem a doença e, consequentemente, não deveria apresentar o sintoma, mas relata apresentá-lo com uma probabilidade $c_{i}$. Uma outra interpretação possível seria a prevalência do sintoma em uma população sem a doença. Devido à dificuldade na interpretação de tal parâmetro, o modelo (2.1) é o menos adotado para a elaboração e validação de instrumentos psiquiátricos e psicológicos. Ainda assim, autores como Coleman, Matthysse, Levy, Cook, Lo, Rubin \& Holzman (2002), por exemplo, aplicaram-no ajustando o modelo logístico de 3 parâmetros a um conjunto de dados que avalia a memória em pacientes esquizofrênicos, obtendo resultados coerentes e interpretáveis clinicamente.

Outras aplicações da TRI na Psiquiatria consideram o ajuste do modelo logístico de 2 parâmetros (2.2). Kessler et al. (2002) ajustam-no a respostas a um inventário de saúde mental para uma amostra de americanos; Schaeffer (1988), ao BDI, e Langenbucher, Labouvie, Sanjuan, Bavly, Martin \& Kirisci (2004), a um instrumento de avaliação de dependência de álcool e drogas. Schaeffer (1988) mostra a diferença entre as distribuições das respostas de homens e mulheres ao item choro do BDI através de um ML2, tornando claro o funcionamento diferencial deste item.

\subsubsection{Aplicação do ML3 a um conjunto de dados psiquiátricos}

Os resultados desta sub-seção referem-se à aplicação do ML3 (com D=1) a dados de depressão do BDI obtidos de Wang et al. (2005). O método adotado para a estimação dos parâmetros de item foi o método bayesiano marginal utilizando a moda a posteriori como estimador. As distribuições a priori consideradas para os parâmetros $a_{i}, b_{i}, c_{i}$ e $\theta_{j}$, $\mathrm{i}=1, \ldots, 21$ e $\mathrm{j}=, 1, \ldots, 208$, foram $\log \operatorname{Normal}(0,4), \operatorname{Normal}(0,4), \operatorname{Beta}(1,3) \mathrm{b}^{3}$ e $\operatorname{Normal}(0,1)$, respectivamente. Os resultados encontram-se na Tabela 2.1 .

Pelas estimativas dos parâmetros de discriminação $a_{i}$ (Tabela 2.1 e Figura 2.7), podese perceber que todos os itens do BDI discriminam relativamente bem os indivíduos (o

\footnotetext{
${ }^{2}$ Repare que $P\left(X_{i j}=1 \mid \theta_{j}=b_{i}, \boldsymbol{\zeta}_{i}\right)=\frac{1+c_{i}}{2}\left(\right.$ se $c_{i}=0$, será a gravidade onde $P\left(X_{i j}=1 \mid \theta_{j}=b_{i}, \boldsymbol{\zeta}_{i}\right)=$ $0,5)$

${ }^{3} \mathrm{~A}$ distribuição foi definida de tal forma que os valores esperados são dados por $E\left[c_{i}\right]=0.25$
} 

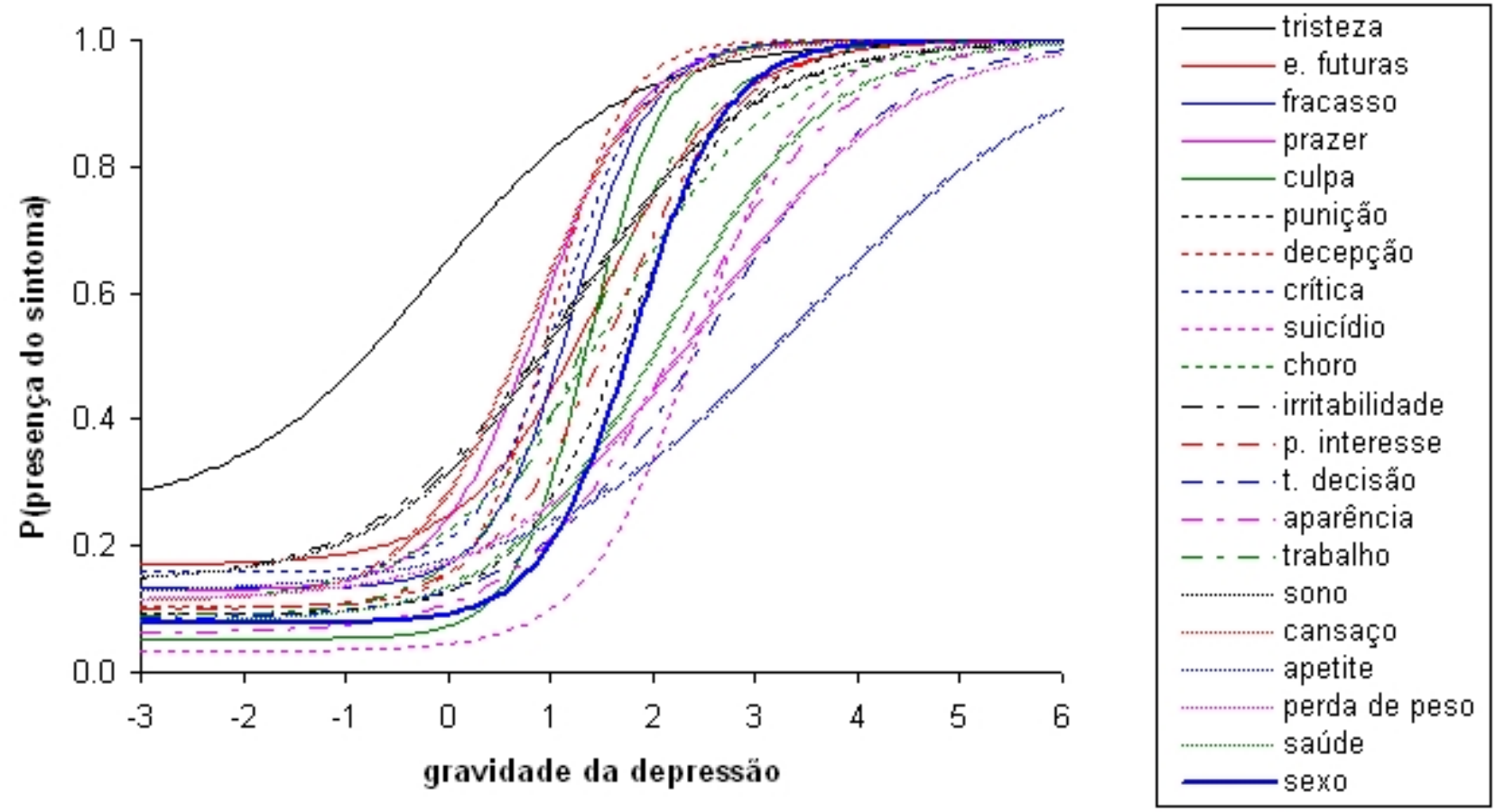

Figura 2.7: CCI dos 21 itens do BDI com parâmetros obtidos do ajuste de um ML3 para os dados de Wang et al. (2005).

menor valor estimado para $a_{i}$, correspondente ao sintoma "apetite", é igual a 0,775 e os demais estão acima de 1). Os itens mais discriminantes são aqueles relativos a sintomas de "sensação de fracasso", "culpa"e "decepção". Os itens 3 (fracasso), 5 (culpa), 6 (punição), 8 (crítica), 17 (cansaço) e 21 (sexo) foram identificados como itens âncora nos níveis de gravidade de depressão iguais a 1,$5 ; 1,5 ; 2,0 ; 1,5 ; 1,0$ e 2,0, respectivamente, quando avaliados níveis de gravidade no intervalo $[-3 ; 3]$ com intervalos de $\delta=1$.

Pelas estimativas de gravidade da depressão $\left(b_{i}\right)$, observa-se que o sintoma "tristeza" (item 1) está presente em níveis bastante tênues de intensidade de depressão, podendo até ser considerada uma característica de prevalência significativa mesmo em indivíduos não deprimidos $(\mathrm{c}=0,250)$. Curiosamente, o sintoma correspondente à maior gravidade de depressão é "perda de apetite" $\left(b_{i}=3.478\right)$. Lembrando que sua capacidade de discriminação é das piores, talvez, este fato não deva ser considerado demasiadamente importante. A Figura 2.8 representa, à direita, as estimativas de gravidade de depressão com o intuito de apontar os níveis de depressão representados pelos 21 itens. As estimativas dos parâmetros $c_{i}$ (Figura 2.8, à esquerda, e Tabela 2.1) indicam, mais uma vez, que o sintoma "tristeza"é o mais comumente relatado, mesmo em níveis baixos de intensidade de depressão, seguido dos sintomas "expectativas futuras"e "crítica". Os demais sintomas apresentam-se com 
Tabela 2.1: Parâmetros do ML3 ajustados para os dados do BDI de Wang et al. (2005)

\begin{tabular}{|c|c|c|c|c|c|c|c|}
\hline \multicolumn{2}{|c|}{ Item } & $\mathrm{a}$ & $\mathrm{EP}(\mathrm{a})$ & $\mathrm{b}$ & $\mathrm{EP}(\mathrm{b})$ & $\mathrm{c}$ & $\mathrm{EP}(\mathrm{c})$ \\
\hline tristeza & BDI01 & 1.042 & 0.281 & -0.145 & 0.344 & 0.250 & 0.093 \\
\hline e. futuras & $\mathrm{BDI} 02^{* *}$ & 1.555 & 0.599 & 1.461 & 0.274 & 0.170 & 0.051 \\
\hline fracasso & BDI03** & 2.536 & 1.110 & 1.206 & 0.164 & 0.132 & 0.040 \\
\hline prazer & BDI04 & 2.094 & 0.635 & 0.901 & 0.174 & 0.129 & 0.048 \\
\hline culpa & BDI05** & 2.785 & 1.083 & 1.378 & 0.148 & 0.053 & 0.022 \\
\hline punição & BDI06** & 1.811 & 0.717 & 1.771 & 0.261 & 0.092 & 0.031 \\
\hline decepção & BDI07 & 2.827 & 1.095 & 0.995 & 0.140 & 0.105 & 0.037 \\
\hline crítica & BDI08** & 2.449 & 1.043 & 1.107 & 0.169 & 0.158 & 0.045 \\
\hline suicídio & BDI09*** & 1.854 & 0.866 & 2.420 & 0.473 & 0.034 & 0.015 \\
\hline choro & BDI10** & 1.219 & 0.362 & 1.579 & 0.300 & 0.111 & 0.044 \\
\hline irritabilidade & BDI11 & 1.098 & 0.290 & 1.151 & 0.283 & 0.141 & 0.056 \\
\hline p. interesse & $\mathrm{BDI} 12^{* *}$ & 1.683 & 0.590 & 1.616 & 0.246 & 0.100 & 0.035 \\
\hline t. decisão & BDI13*** & 1.162 & 0.453 & 2.569 & 0.614 & 0.084 & 0.030 \\
\hline aparência & BDI14*** & 1.300 & 0.470 & 2.285 & 0.475 & 0.062 & 0.025 \\
\hline trabalho & BDI15** & 1.680 & 0.500 & 1.392 & 0.198 & 0.090 & 0.035 \\
\hline sono & BDI16** & 1.152 & 0.320 & 1.204 & 0.285 & 0.145 & 0.056 \\
\hline cansaço & BDI17* & 1.801 & 0.431 & 0.798 & 0.173 & 0.114 & 0.046 \\
\hline apetite & BDI18*** & 0.775 & 0.297 & 3.478 & 1.171 & 0.124 & 0.040 \\
\hline perda de peso & BDI19*** & 1.043 & 0.391 & 2.507 & 0.622 & 0.114 & 0.039 \\
\hline saúde & BDI20*** & 1.285 & 0.448 & 2.131 & 0.418 & 0.080 & 0.031 \\
\hline sexo & $\mathrm{BDI} 21^{* *}$ & 2.223 & 1.007 & 1.825 & 0.245 & 0.077 & 0.024 \\
\hline
\end{tabular}

EP: erro padrão; a: parämetro de discriminação; b: parâmetro de gravidade de depressão;

c: probabilidade de presença de sintoma em indivíduos sem depressão; *: itens âncora no nível $\theta=1$

**: itens âncora no nível $\theta=2 ; * * *$ : itens âncora no nível $\theta=3$. 
freqüência relativamente baixa para baixos níveis de gravidade da depressão, conforme esperado.

Os erros padrão das estimativas dos itens são relativamente grandes em função de o tamanho amostral ser menor que aquele recomendado por estudos de simulação, em torno de 500 indivíduos. Uma alternativa para diminuição dos erros padrão, seria a adoção de modelos com um menor número de parâmetros de itens (ML1 ou ML2), caso tenham uma interpretação plausível e sejam apropriados para os dados em questão.

\subsubsection{Comparação entre a teoria clássica e o ML3 da TRI no es- tudo da qualidade de instrumentos de avaliação psiquiátrica}

Para ilustrar a aplicabilidade da TRI no estudo da qualidade dos instrumentos de avaliação psiquiátrica, é fundamental que os aspectos de confiabilidade, validade, consistência interna e dimensionalidade possam ser avaliados.

Uma visualização de como tais aspectos podem ser estudados com os resultados obtidos no ML3 da TRI será feita comparando-os com aqueles provenientes da análise clássica. Neste contexto, as seguintes analogias podem ser citadas:

- O escore total pode ser representado pelo parâmetro de indivíduo $\theta_{j}$. A grande desvantagem do escore total, quando comparado à gravidade da doença $\theta_{j}$ da TRI, é que ele é uma medida da quantidade de sintomas apresentados pelo indivíduo (soma dos escores de cada item). A diferenciação de sintomas graves ou não graves não é considerada nesse cálculo;

- O estudo da confiabilidade do instrumento pode ser realizado utilizando-se a análise clássica (Kappa, Kappa ponderado ou coeficiente de correlação intraclasse) com as estimativas do traço latente $\theta_{j}$, ao invés do escore total;

- Na verificação da validade do instrumento, a análise discriminante e a regressão logística, buscam identificar quais são os itens mais importantes na diferenciação dos indivíduos e quais são aqueles que não acrescentam nenhuma informação para tal diferenciação. Pelo modelo ML3 da TRI, os parâmetros $a_{i}$ são relacionados a essa informação;

- Para a verificação da consistência interna do instrumento, os coeficientes alfa de Cronbach e as correlações item-total (correlações bisseriais) poderiam ser substituídos pelos parâmetros $a_{i}$, que quantificam a capacidade de o item avaliar a gravidade de depressão;

- O estudo de dimensionalidade do instrumento também pode ser realizado com a TRI, embora este aspecto não tenha sido abordado anteriormente por não fazer 
$c_{i}$

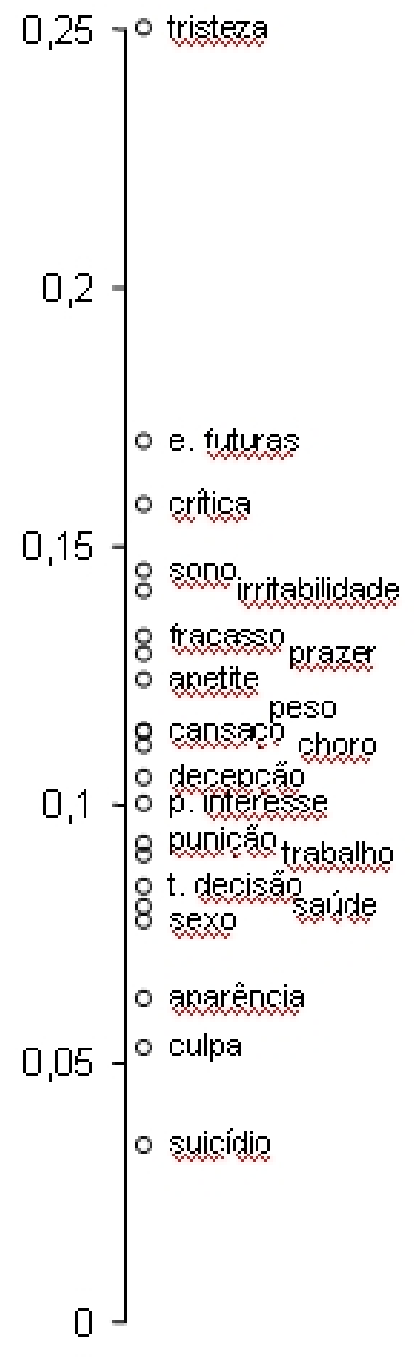

$\mathbf{b}_{\mathrm{i}}$

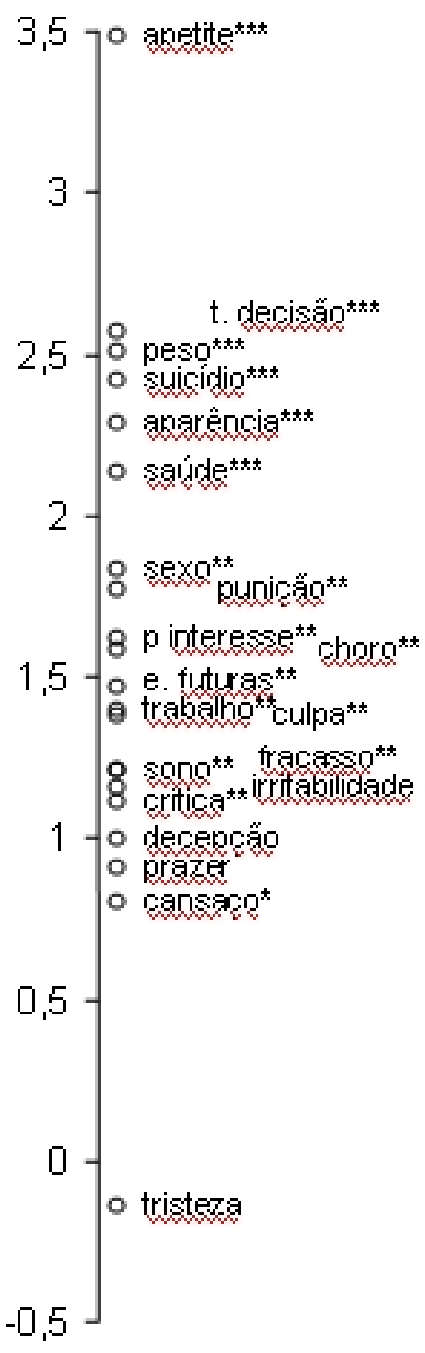

Figura 2.8: Estimativa dos parâmetros de gravidade (b) e probabilidade de presença de sintoma em indivíduos sem depressão (c) dos 21 itens do BDI ajustados pelo ML3 para os dados de Wang et al. (2005). (*: itens âncora no nível $\theta=1$; **: itens âncora no nível $\theta=2 \mathrm{e}^{* * *}$ : itens âncora no nível $\theta=3$ ). 
parte dos objetivos específicos deste trabalho. Detalhes dessa abordagem podem ser encontrados em Nojosa (2002);

- Os parâmetros $c_{i}$ podem representar as prevalências dos sintomas numa população sem doença.

Além disso, a vantagem da TRI em separar os parâmetros associados aos indivíduos e aos itens e a interpretação do parâmetro $b_{i}$ não têm nenhuma analogia com a análise clássica. 


\section{Capítulo 3}

\section{Modelo da TRI para Itens Constrangedores}

Determinados itens têm a característica de intimidar ou constranger alguns indivíduos, levando-os, possivelmente, a mentir em suas respostas. Tendo este fato em mente, propôs-se um modelo que possibilita diferenciar a gravidade da presença do sintoma entre indivíduos que são ou não constrangidos pelo item correspondente. Tome, por exemplo, o item choro do BDI (item 10). Suponha que os homens caracterizam o grupo constrangido pelo choro e que as mulheres representam o grupo não constrangido. O fato de um homem não conseguir evitar o choro pode refletir um nível de depressão mais grave do que o de uma mulher, cujo choro pode estar presente em níveis brandos ou até inexistentes de depressão. O modelo procura levar em consideração possíveis diferenças na probabilidade de presença do sintoma dos dois grupos (item com funcionamento diferencial).

Suponha que $\mathrm{n}$ indivíduos responderam os I itens do instrumento e que $X_{i j}$ é uma variável dicotômica que assume os valores 1 , quando o indivíduo j relata apresentar o sintoma descrito no item i, e 0 em caso contrário, $C_{i j}$ é uma variável dicotômica que assume os valores 1 , quando o indivíduo $\mathrm{j}$ sente-se constrangido pela pergunta do item i (e, possivelmente, mentiria em sua resposta), e 0 em caso contrário e $\theta_{j}$ é o nível do traço latente do j-ésimo indivíduo, indicando a gravidade da doença. O modelo proposto determina que a probabilidade de o indivíduo $\mathrm{j}$, não constrangido pelo item i, relatar a presença de tal sintoma depende de sua gravidade $\left(\theta_{j}\right)$ segundo o modelo logístico de 2 parâmetros, que pode ser escrito como

$$
P\left(X_{i j}=1 \mid C_{i j}=0, \theta_{j}, a_{i}, b_{1 i}\right)=\frac{1}{1+e^{-a_{i}\left(\theta_{j}-b_{1 i}\right)}}=P_{i j},
$$

em que $a_{i}$ e $b_{1 i}$ são os parâmetros de discriminação e de gravidade, respectivamente. Por outro lado, a probabilidade de o indivíduo j, constrangido pelo item i, relatar a presença de 
tal sintoma também depende da gravidade da doença $\left(\theta_{j}\right)$ segundo o modelo logístico de 2 parâmetros mas com parâmetro de gravidade diferente daquele que integra o modelo para sujeitos não constrangidos, ou seja, $P\left(X_{i j}=1 \mid C_{i j}=1, \theta_{j}, a_{i}, b_{2 i}\right)=\left[1+e^{-a_{i}\left(\theta_{j}-b_{2 i}\right)}\right]^{-1}$. Note que o parâmetro de discriminação $\left(a_{i}\right)$ é comum aos dois grupos, mas que o de gravidade, $b_{1 i}$, para o grupo não constrangido, e $b_{2 i}$, para o grupo constrangido, não. Porém, é importante lembrar que no grupo de constrangidos pelo item i, alguns indivíduos podem relatar que não apresentam o sintoma quando, na realidade, o apresentam. Desta maneira, um parâmetro de erro de classificação da resposta $X_{i j}$ deve ser incorporado ao modelo devido ao constrangimento e possível mentira neste grupo. Está-se supondo que a resposta do indivíduo $\left(X_{i j}\right)$ pode não representar a verdade (apenas no grupo de constrangidos). Seja $U_{i j}$ uma variável dicotômica, que assume os valores 1, quando o indivíduo constrangido j realmente apresenta o sintoma i e 0 em caso contrário. Pode-se escrever que:

$$
\begin{aligned}
P & \left(X_{i j}=1 \mid C_{i j}=1, \theta_{j}, a_{i}, b_{2 i}, \gamma_{i}\right)= \\
= & P\left(X_{i j}=1, U_{i j}=1 \mid C_{i j}=1, \theta_{j}, a_{i}, b_{2 i}, \gamma_{i}\right)+P\left(X_{i j}=1, U_{i j}=0 \mid C_{i j}=1, \theta_{j}, a_{i}, b_{2 i}, \gamma_{i}\right) \\
= & P\left(X_{i j}=1 \mid U_{i j}=1, C_{i j}=1, \theta_{j}, a_{i}, b_{2 i}, \gamma_{i}\right) P\left(U_{i j}=1 \mid C_{i j}=1, \theta_{j}, a_{i}, b_{2 i}, \gamma_{i}\right)+ \\
& P\left(X_{i j}=1 \mid U_{i j}=0, C_{i j}=1, \theta_{j}, a_{i}, b_{2 i}, \gamma_{i}\right) P\left(U_{i j}=0 \mid C_{i j}=1, \theta_{j}, a_{i}, b_{2 i}, \gamma_{i}\right) \\
= & \gamma_{i} P\left(U_{i j}=1 \mid C_{i j}=1, \theta_{j}, a_{i}, b_{2 i}, \gamma_{i}\right)+0 P\left(U_{i j}=0 \mid C_{i j}=1, \theta_{j}, a_{i}, b_{2 i}, \gamma_{i}\right) \\
= & \frac{\gamma_{i}}{1+e^{-a_{i}\left(\theta_{j}-b_{2 i}\right)}}=P_{i j}^{*},
\end{aligned}
$$

em que se considera que $P\left(U_{i j}=1 \mid C_{i j}=1, \theta_{j}, a_{i}, b_{2 i}, \gamma_{i}\right)$ segue o modelo logístico de dois parâmetros $\left(a_{i}\right.$ e $\left.b_{2 i}\right)$, que o indivíduo constrangido não mente no sentido de dizer que tem o sintoma dado que não o tem $\left(P\left(X_{i j}=1 \mid U_{i j}=0, C_{i j}=1, \theta_{j}, a_{i}, b_{2 i}, \gamma_{i}\right)=0\right)$ e que a probabilidade de um indivíduo constrangido dizer que tem o sintoma dado que realmente o apresenta é igual a $0 \leq \gamma_{i} \leq 1$.

Considerando que a probabilidade do item i constranger o indivíduo j não depende de $\theta_{j}$ e é igual a $\delta_{i}$, o modelo proposto define a função de probabilidade conjunta das respostas $X_{i j}$ e $C_{i j}$, condicionada a $\theta_{j}$, como:

$$
\begin{aligned}
& P\left(X_{i j}=x_{i j}, C_{i j}=c_{i j} \mid \theta_{j}, \boldsymbol{\zeta}\right)=P\left(X_{i j}=x_{i j} \mid C_{i j}=c_{i j}, \theta_{j}, \boldsymbol{\zeta}\right) P\left(C_{i j}=c_{i j} \mid \theta_{j}, \boldsymbol{\zeta}\right) \\
& \quad=\left[\left(P_{i j}^{*}\right)^{x_{i j}}\left(1-P_{i j}^{*}\right)^{\left(1-x_{i j}\right)} \delta_{i}\right]^{c_{i j}} \times\left[P_{i j}^{x_{i j}}\left(1-P_{i j}\right)^{\left(1-x_{i j}\right)}\left(1-\delta_{i}\right)\right]^{1-c_{i j}}
\end{aligned}
$$

$\operatorname{com} \boldsymbol{\zeta}=\left(\boldsymbol{\zeta}_{1}, \ldots, \boldsymbol{\zeta}_{I}\right)^{t}$ e $\boldsymbol{\zeta}_{i}=\left(a_{i}, b_{1 i}, b_{2 i}, \gamma_{i}, \delta_{i}\right)^{t}$. Mais especificamente, o modelo (3.3) para todos os possíveis valores de $x_{i j}$ e $c_{i j}$ é:

$P\left(X_{i j}=1, C_{i j}=1 \mid \theta_{j}, \boldsymbol{\zeta}\right)=P_{i j}^{*} \delta_{i}$

$P\left(X_{i j}=0, C_{i j}=1 \mid \theta_{j}, \boldsymbol{\zeta}\right)=\left(1-P_{i j}^{*}\right) \delta_{i}$

$P\left(X_{i j}=1, C_{i j}=0 \mid \theta_{j}, \boldsymbol{\zeta}\right)=P_{i j}\left(1-\delta_{i}\right)$

$P\left(X_{i j}=0, C_{i j}=0 \mid \theta_{j}, \boldsymbol{\zeta}\right)=\left(1-P_{i j}\right)\left(1-\delta_{i}\right)$

Repare que se $\delta_{i}=0, i=1, \ldots, I$, o modelo (3.3) reduz-se ao ML2 (2.2). Por outro lado, se $\delta_{i}=1, i=1, \ldots, I$, o modelo (3.3) reduz-se ao ML4 (2.4) com $c_{i}=0$. 
Atualmente, os instrumentos psiquiátricos existentes incluem apenas a variável $X_{i j}$, mas não a variável $C_{i j}$. É claro que há situações em que o pesquisador consegue deduzir, com base em pesquisas anteriores ou em sua experiência clínica, para quais indivíduos j e itens i, $C_{i j}$ assume valor 1 . Por exemplo, Schaeffer (1988) mostra, através da aplicação do ML2 a uma amostra de 1710 americanos respondedores do BDI, que o item choro $\left(n^{o} 10\right)$ apresenta relação diferente com a gravidade da depressão $\left(\theta_{j}\right)$ entre homens e mulheres. Através do ajuste dos parâmetros do ML2 separadamente para os dois sexos, inferiu que o parâmetro de gravidade $\left(b_{i}\right)$ do item choro era maior no sexo masculino do que no feminino. Os demais parâmetros não diferiam significativamente. Um outro exemplo é o inventário de triagem do uso de drogas (DUSI). Ele é composto por 149 itens dicotômicos (sintoma presente ou ausente) subdivididos em 10 sub-áreas (uso de substância, comportamento, saúde, transtornos psiquiátricos, sociabilidade, família, escola, trabalho, relações com amigos e lazer). Uma reformulação do inventário (DUSI-R, apresentado no Apêndice B) foi proposta por Kirisci, Hsu \& Tarter (1994) na tentativa de identificar possíveis falsas respostas aos 149 itens originais. Essa reformulação consiste na inclusão de 1 item, relacionado à vida cotidiana do indivíduo, ao final de cada sub-área (10 itens extras, no total). Tais itens não apresentam nenhuma relação com o uso de drogas, mas sim associações com atitudes consideradas comuns e presentes no comportamento do ser humano. A negação da presença dessas atitudes pelo indivíduo, pode significar um perfil incongruente (ou mentiroso) das respostas dadas aos itens originais da respectiva sub-área. Um indivíduo que negou a ocorrência do item extra de uma determinada sub-área, pode ser alocado no grupo de constrangidos (ou possíveis mentirosos) para todos os itens originais pertencentes a ela.

Outras situações em que é possível adotar o modelo (3.3) são os estudos em que o instrumento é respondido por duas pessoas: o próprio paciente e seu responsável (um familiar, por exemplo), avaliando o paciente. Pesquisas desse tipo são muito comuns quando a amostra de pacientes é composta por crianças. Para cada item i, a resposta $C_{i j}$ do paciente pode ser considerada 1 se sua resposta $X_{i j}$ não for congruente com a de seu responsável e 0, em caso contrário.

As sugestões acima são apenas alternativas de utilização do modelo (3.3). A incorporação, aos instrumentos psiquiátricos, de perguntas identificadoras das respostas $C_{i j}$ poderia ser mais conveniente. A necessidade de inclusão dessa pergunta para cada um dos itens do instrumento ou apenas para parte deles deve ser discutida juntamente com o psiquiatra. Como não introduz alterações substanciais no instrumento, sua inclusão é perfeitamente factível. 


\section{Capítulo 4}

\section{Estimação}

Há, basicamente, três situações que podem ser consideradas no processo de estimação: aquelas em que os parâmetros de itens são conhecidos e os traços latentes dos indivíduos são desconhecidos; aquelas em que os parâmetros de itens são desconhecidos e os traços latentes dos indivíduos são conhecidos; e aquelas em que ambos os tipos de parâmetros são desconhecidos.

Por não ser uma situação encontrada na área de Psiquiatria, não se considerará a situação em que traços latentes dos indivíduos são conhecidos 1. Na seção 4.1, o método de máxima verossimilhança será adotado para a estimação dos traços latentes no caso de parâmetros de itens conhecidos. Nas seções 4.2 e 4.3 , os métodos de máxima verossimilhança marginal e bayesiano (por simulação MCMC), respectivamente, serão propostos para a estimação de ambos os tipos de parâmetros. O método de máxima verossimilhança conjunta não será discutido por ser extremamente trabalhoso computacionalmente e requerer um tempo de processamento muito grande.

\subsection{Itens Conhecidos - Máxima Verossimilhança (MV)}

Em alguns casos, os itens do instrumento de avaliação psiquiátrica podem ser considerados conhecidos se seus parâmetros já tiverem sido estimados anteriormente. É claro que a amostra de indivíduos em questão deve ser comparável (com mesmas características clínicas e demográficas) à amostra adotada para a estimação prévia. Neste contexto, o método de máxima verossimilhança pode ser utilizado para a estimação dos parâmetros

\footnotetext{
${ }^{1}$ Se dados sob investigação tiverem esta característica, os estimadores dos parâmetros dos itens podem ser obtidos através das equações 4.40 e 4.41.
} 
individuais, $\theta_{j}$.

Supondo independência entre as respostas de diferentes indivíduos e independência entre as respostas de cada indivíduo aos diferentes itens, condicionalmente ao traço latente $\left(\theta_{j}\right)$, pode-se escrever a função de verossimilhança do modelo 3.3 como:

$$
\begin{aligned}
L(\boldsymbol{\theta}) & =\prod_{j=1}^{n} P\left(\boldsymbol{X}_{\cdot j}=\boldsymbol{x}_{\cdot j}, \boldsymbol{C}_{\cdot j}=\boldsymbol{c}_{\cdot j} \mid \theta_{j}, \boldsymbol{\zeta}\right)=\prod_{j=1}^{n} \prod_{i=1}^{I} P\left(X_{i j}=x_{i j}, C_{i j}=c_{i j} \mid \theta_{j}, \boldsymbol{\zeta}_{i}\right) \\
& =\prod_{i=1}^{I} \prod_{j=1}^{n}\left[\left(P_{i j}^{*}\right)^{x_{i j}}\left(1-P_{i j}^{*}\right)^{\left(1-x_{i j}\right)} \delta_{i}\right]^{c_{i j}} \times\left[P_{i j}^{x_{i j}}\left(1-P_{i j}\right)^{\left(1-x_{i j}\right)}\left(1-\delta_{i}\right)\right]^{1-c_{i j}}
\end{aligned}
$$

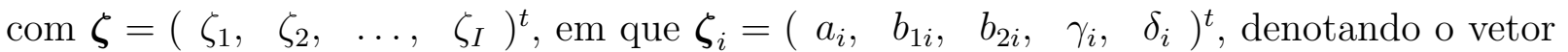
de todos dos parâmetros dos itens e $P_{i j}$ e $P_{i j}^{*}$ dados, respectivamente, em (3.1) e 3.2.

A log-verossimilhança é

$$
\begin{aligned}
l(\boldsymbol{\theta})= & \sum_{i=1}^{I} \sum_{j=1}^{n}\left[x_{i j} c_{i j} \log P_{i j}^{*}+\left(1-x_{i j}\right) c_{i j} \log \left(1-P_{i j}^{*}\right)+c_{i j} \log \delta_{i}+\right. \\
& +x_{i j}\left(1-c_{i j}\right) \log P_{i j}+\left(1-x_{i j}\right)\left(1-c_{i j}\right) \log \left(1-P_{i j}\right)+ \\
& \left.+\left(1-c_{i j}\right) \log \left(1-\delta_{i}\right)\right]
\end{aligned}
$$

em que $P_{i j}$ e $P_{i j}^{*}$ são obtidos das equações 3.1 e 3.2 , respectivamente.

Derivando 4.2 com respeito a $\theta_{j}$, obtém-se:

$$
\begin{aligned}
\frac{\partial l(\boldsymbol{\theta})}{\partial \theta_{j}}= & \sum_{i=1}^{I}\left[x_{i j} c_{i j} \frac{\partial \log P_{i j}^{*}}{\partial \theta_{j}}+\left(1-x_{i j}\right) c_{i j} \frac{\partial \log \left(1-P_{i j}^{*}\right)}{\partial \theta_{j}}+c_{i j} \frac{\partial \log \delta_{i}}{\partial \theta_{j}}+\right. \\
& +x_{i j}\left(1-c_{i j}\right) \frac{\partial \log P_{i j}}{\partial \theta_{j}}+\left(1-x_{i j}\right)\left(1-c_{i j}\right) \frac{\partial \log \left(1-P_{i j}\right)}{\partial \theta_{j}}+ \\
& \left.+\left(1-c_{i j}\right) \frac{\partial \log \left(1-\delta_{i}\right)}{\partial \theta_{j}}\right] \\
= & \sum_{i=1}^{I}\left\{\left[\frac{x_{i j} c_{i j}}{P_{i j}^{*}}-\frac{\left(1-x_{i j}\right) c_{i j}}{1-P_{i j}^{*}}\right] \frac{\partial P_{i j}^{*}}{\partial \theta_{j}}+\left[\frac{c_{i j}}{\delta_{i}}-\frac{\left(1-c_{i j}\right)}{1-\delta_{i}}\right] \frac{\partial \delta_{i}}{\partial \theta_{j}}\right. \\
& \left.+\left[\frac{x_{i j}\left(1-c_{i j}\right)}{P_{i j}}-\frac{\left(1-x_{i j}\right)\left(1-c_{i j}\right)}{1-P_{i j}}\right] \frac{\partial P_{i j}}{\partial \theta_{j}}\right\} .
\end{aligned}
$$

Substituindo as derivadas explicitadas em A.1, A.2 e A.3), do Apêncice A, na equação 4.3), tem-se: 


$$
\frac{\partial l(\boldsymbol{\theta})}{\partial \theta_{j}}=\sum_{i=1}^{I}\left[\frac{c_{i j}\left(x_{i j}-P_{i j}^{*}\right) a_{i}\left(\gamma_{i}-P_{i j}^{*}\right)}{\gamma_{i}\left(1-P_{i j}^{*}\right)}+\left(1-c_{i j}\right)\left(x_{i j}-P_{i j}\right) a_{i}\right] .
$$

Então, a equação de estimação de $\theta_{j}$ é

$$
\sum_{i=1}^{I}\left[\frac{c_{i j}\left(x_{i j}-P_{i j}^{*}\right) a_{i}\left(\gamma_{i}-P_{i j}^{*}\right)}{\gamma_{i}\left(1-P_{i j}^{*}\right)}+\left(1-c_{i j}\right)\left(x_{i j}-P_{i j}\right) a_{i}\right]=0
$$

Por não apresentar solução explícita, algum processo iterativo como Newton-Raphson ou Scoring de Fisher deve ser adotado para a solução de (4.5). Para tanto, a expressão da segunda derivada de $l(\boldsymbol{\theta})$ em relação a $\theta_{j}$ é necessária. Pelas expressões (A.4), A.5) e A.6., explicitadas no Apêndice A, segue que:

$$
\begin{aligned}
\frac{\partial^{2} l(\boldsymbol{\theta})}{\partial \theta_{j}^{2}}= & \sum_{i=1}^{I}\left\{\frac{a_{i}^{2}}{\gamma_{i}^{2}} \frac{\left(\gamma_{i}-P_{i j}^{*}\right)}{\left(1-P_{i j}^{*}\right)}\left(x_{i j}-P_{i j}^{*}\right) c_{i j}\left[\frac{\left(\gamma_{i}-P_{i j}^{*}\right)\left(1-x_{i j}\right)}{1-P_{i j}^{*}}-P_{i j}^{*}\right]+\right. \\
& \left.+a_{i}^{2}\left(1-c_{i j}\right)\left(x_{i j}-P_{i j}\right)\left(1-x_{i j}-P_{i j}\right)\right\} .
\end{aligned}
$$

Para o cálculo dos erros padrão dos estimadores dos parâmetros dos itens e para a utilização do algoritmo Scoring de Fisher, a esperança de (4.6) deve ser obtida. Lembrando que $C_{i j} \sim \operatorname{Bernoulli}\left(\delta_{i}\right), X_{i j}\left|C_{i j}=1 \sim \operatorname{Bernoulli}\left(P_{i j}^{*}\right), X_{i j}\right| C_{i j}=0 \sim \operatorname{Bernoulli}\left(P_{i j}\right)$ e que, portanto, $E\left[C_{i j}\right]=\delta_{i}, \quad E\left[X_{i j} C_{i j}\right]=P\left(X_{i j}=1 \mid C_{i j}=1\right) P\left(C_{i j}=1\right)=$ $P_{i j}^{*} \delta_{i}$ e $E\left[X_{i j}\right]=E\left[E\left(X_{i j} \mid C_{i j}\right)\right]=P_{i j}^{*} \delta_{i}+P_{i j}\left(1-\delta_{i}\right)$, tem-se

$$
E\left[\frac{\partial^{2} l(\boldsymbol{\theta})}{\partial \theta_{j}^{2}}\right]=\sum_{i=1}^{I}\left\{\frac{-\delta_{i}}{\left(1-P_{i j}^{*}\right)} \frac{a_{i}^{2}}{\gamma_{i}^{2}} P_{i j}^{*}\left(\gamma_{i}-P_{i j}^{*}\right)^{2}-\left(1-\delta_{i}\right) a_{i}^{2} P_{i j}\left(1-P_{i j}\right)\right\}
$$

Sob algumas condições de regularidade (Sen \& Singer 1993), o estimador de MV $\hat{\theta}_{j}$ tem distribuição assintoticamente Normal com média $\theta_{j}$ e variância dada pela inversa da matriz de informação de Fisher, ou seja, $\hat{\theta_{j}} \approx N\left(\theta_{j},-\left[E\left(\frac{\partial^{2} l(\boldsymbol{\theta})}{\partial \theta_{j}^{2}}\right)\right]^{-1}\right)$. 


\subsection{Itens e Habilidades Desconhecidos - MV Marginal (MVM)}

A estimação por MVM é dividida em duas etapas. Inicialmente, estimam-se os parâmetros dos itens adotando-se um artifício para eliminar os parâmetros $\theta_{j}$ da função de verossimilhança. Uma vez obtidas as estimativas dos parâmetros dos itens, a estimação dos traços latentes $\theta_{j}$ é realizada seguindo a metodologia descrita na seção 4.1, supondo que as estimativas correspondem aos verdadeiros valores dos parâmetros.

Nesta seção, duas abordagens serão mencionadas para estimação dos parâmetros dos itens. A primeira, com base na proposta de Bock \& Lieberman (1970), consiste na estimação de todos os itens simultaneamente. A segunda, inspirada na formulação de Bock \& Aitkin (1981), permite a estimação isolada dos parâmetros de cada item.

\subsubsection{Estimação de $\zeta_{i}$ para todos os itens simultaneamente}

Para a eliminação dos parâmetros individuais $\left(\theta_{j}\right)$ da verossimilhança, considera-se $\left\{\theta_{j}, j=1, \ldots, n\right\}$ como uma amostra de uma variável aleatória contínua $\theta$ com função densidade de probabilidade $g(\theta \mid \boldsymbol{\eta})$, derivável até segunda ordem e com hiperparâmetro

$\boldsymbol{\eta}$ conhecido. Nos casos em que $\theta_{j}$ tem distribuição Normal, $\boldsymbol{\eta}=\left(\mu, \sigma^{2}\right)$, a média $\mu$ e a variância $\sigma^{2}$ do traço latente na população são escolhidos de acordo com a métrica que se deseja adotar.

A probabilidade marginal conjunta de $\boldsymbol{X}_{\cdot j}=\left(X_{1 j}, X_{2 j}, \ldots, X_{I j}\right)$ e $\boldsymbol{C}_{\cdot j}=\left(C_{1 j}, C_{2 j}, \ldots, C_{I j}\right)$ pode ser escrita como:

$$
\begin{aligned}
P\left(\boldsymbol{X}_{\cdot j}=\boldsymbol{x}_{\cdot j}, \boldsymbol{C}_{\cdot j}=\boldsymbol{c}_{\cdot j} \mid \boldsymbol{\zeta}, \boldsymbol{\eta}\right) & =\int_{\mathbb{R}} P\left(\boldsymbol{X}_{\cdot j}=\boldsymbol{x}_{\cdot j}, \boldsymbol{C}_{\cdot j}=\boldsymbol{c}_{\cdot j} \mid \theta, \boldsymbol{\zeta}, \boldsymbol{\eta}\right) g(\theta \mid \boldsymbol{\eta}) d \theta \\
& =\int_{\mathbb{R}} P\left(\boldsymbol{X}_{\cdot j}=\boldsymbol{x}_{\cdot j}, \boldsymbol{C}_{\cdot j}=\boldsymbol{c}_{\cdot j} \mid \theta, \boldsymbol{\zeta}\right) g(\theta \mid \boldsymbol{\eta}) d \theta \\
& =\int_{\mathbb{R}} \prod_{i=1}^{I} P\left(X_{i j}=x_{i j}, C_{i j}=c_{i j} \mid \theta, \boldsymbol{\zeta}\right) g(\theta \mid \boldsymbol{\eta}) d \theta
\end{aligned}
$$

Como as respostas de diferentes indivíduos são independentes, a função de verossimilhança marginal é

$$
L(\boldsymbol{\zeta} \mid \boldsymbol{\eta})=\prod_{j=1}^{n} \int_{\mathbb{R}} \prod_{i=1}^{I} P\left(X_{i j}=x_{i j}, C_{i j}=c_{i j} \mid \theta, \boldsymbol{\zeta}\right) g(\theta \mid \boldsymbol{\eta}) d \theta
$$


e, portanto, a log-verossimilhança marginal é

$$
l(\boldsymbol{\zeta} \mid \boldsymbol{\eta})=\sum_{j=1}^{n} \log \int_{\mathbb{R}} \prod_{i=1}^{I} P\left(X_{i j}=x_{i j}, C_{i j}=c_{i j} \mid \theta, \zeta\right) g(\theta \mid \boldsymbol{\eta}) d \theta
$$

Derivando 4.10) com respeito a $\boldsymbol{\zeta}_{i}$ e igualando a expressão resultante a zero, obtém-se as equações de estimação cuja solução corresponde aos estimadores de MVM dos parâmetros dos itens. Explicitamente, tem-se

$$
\begin{aligned}
& \frac{\partial l(\boldsymbol{\zeta} \mid \boldsymbol{\eta})}{\partial \boldsymbol{\zeta}_{i}}=\sum_{j=1}^{n} \frac{\partial \log P\left(\boldsymbol{X}_{\cdot j}=\boldsymbol{x}_{\cdot j}, \boldsymbol{C}_{\cdot j}=\boldsymbol{c}_{\cdot j} \mid \boldsymbol{\zeta}, \boldsymbol{\eta}\right)}{\partial \boldsymbol{\zeta}_{i}} \\
& \quad=\sum_{j=1}^{n} \frac{1}{P\left(\boldsymbol{X}_{\cdot j}=\boldsymbol{x}_{\cdot j}, \boldsymbol{C}_{\cdot j}=\boldsymbol{c}_{\cdot j} \mid \boldsymbol{\zeta}, \boldsymbol{\eta}\right)} \frac{\partial P\left(\boldsymbol{X}_{\cdot j}=\boldsymbol{x}_{\cdot j}, \boldsymbol{C}_{\cdot j}=\boldsymbol{c}_{\cdot j} \mid \boldsymbol{\zeta}, \boldsymbol{\eta}\right)}{\partial \boldsymbol{\zeta}_{i}} .
\end{aligned}
$$

Para simplificar a notação, escreve-se $P\left(\boldsymbol{X}_{\cdot j}=\boldsymbol{x}_{\cdot j}, \boldsymbol{C}_{\cdot j}=\boldsymbol{c}_{\cdot j} \mid \boldsymbol{\zeta}, \boldsymbol{\eta}\right)=P\left(\boldsymbol{x}_{\cdot j}, \boldsymbol{c}_{\cdot j} \mid \boldsymbol{\zeta}, \boldsymbol{\eta}\right)$. Note que

$$
\begin{aligned}
\frac{\partial P\left(\boldsymbol{x}_{\cdot j}, \boldsymbol{c}_{\cdot j} \mid \boldsymbol{\zeta}, \boldsymbol{\eta}\right)}{\partial \boldsymbol{\zeta}_{i}}=\frac{\partial}{\partial \boldsymbol{\zeta}_{i}} \int_{\mathbb{R}} \prod_{t=1}^{I} P\left(X_{t j}=x_{t j}, C_{t j}=c_{t j} \mid \theta, \boldsymbol{\zeta}\right) g(\theta \mid \boldsymbol{\eta}) d \theta \\
=\int_{\mathbb{R}} \frac{\partial}{\partial \boldsymbol{\zeta}_{i}}\left[\prod_{t=1}^{I} P\left(X_{t j}=x_{t j}, C_{t j}=c_{t j} \mid \theta, \boldsymbol{\zeta}\right) g(\theta \mid \boldsymbol{\eta})\right] d \theta \\
=\int_{\mathbb{R}}\left\{\left[\prod_{t=1 \mathrm{e} t \neq i}^{I} P\left(X_{t j}=x_{t j}, C_{t j}=c_{t j} \mid \theta, \boldsymbol{\zeta}\right)\right] \times \frac{\partial P\left(X_{i j}=x_{i j}, C_{i j}=c_{i j} \mid \theta, \boldsymbol{\zeta}\right)}{\partial \boldsymbol{\zeta}_{i}} g(\theta \mid \boldsymbol{\eta})\right\} d \theta \\
=\int_{\mathbb{R}}\left\{\frac{\partial P\left(X_{i j}=x_{i j}, C_{i j}=c_{i j} \mid \theta, \boldsymbol{\zeta}\right) / \partial \boldsymbol{\zeta}_{i}}{P\left(X_{i j}=x_{i j}, C_{i j}=c_{i j} \mid \theta, \boldsymbol{\zeta}\right)} \times P\left(\boldsymbol{x}_{\cdot j}, \boldsymbol{c}_{\cdot j} \mid \theta, \boldsymbol{\zeta}\right) g(\theta \mid \boldsymbol{\eta})\right\} d \theta,
\end{aligned}
$$

em que a derivada e a integral podem ser permutadas com base no Teorema da Convergência Dominada de Lebesgue (Bartle 1966). Tomando $P\left(X_{i j}=x_{i j}, C_{i j}=c_{i j} \mid \theta, \boldsymbol{\zeta}\right)$ como no modelo (3.3) e através dos cálculos explicitados em (A.7), tem-se

$$
\begin{aligned}
& \frac{\partial P\left(X_{i j}=x_{i j}, C_{i j}=c_{i j} \mid \theta, \boldsymbol{\zeta}\right)}{\partial \boldsymbol{\zeta}_{i}}= \\
& =\frac{\partial}{\partial \boldsymbol{\zeta}_{i}}\left\{\left[\left(P_{i}^{*}\right)^{x_{i j}}\left(1-P_{i}^{*}\right)^{\left(1-x_{i j}\right)} \delta_{i}\right]^{c_{i j}} \times\left[P_{i}^{x_{i j}}\left(1-P_{i}\right)^{\left(1-x_{i j}\right)}\left(1-\delta_{i}\right)\right]^{1-c_{i j}}\right\} \\
& =P\left(X_{i j}=x_{i j}, C_{i j}=c_{i j} \mid \theta, \boldsymbol{\zeta}\right)\left[\frac{\partial P_{i}^{*}}{\partial \boldsymbol{\zeta}_{i}} \frac{c_{i j}\left(x_{i j}-P_{i}^{*}\right)}{P_{i}^{*}\left(1-P_{i}^{*}\right)}+\frac{\partial \delta_{i}}{\partial \boldsymbol{\zeta}_{i}} \frac{\left(c_{i j}-\delta_{i}\right)}{\delta_{i}\left(1-\delta_{i}\right)}+\frac{\partial P_{i}}{\partial \boldsymbol{\zeta}_{i}} \frac{\left(1-c_{i j}\right)\left(x_{i j}-P_{i}\right)}{P_{i}\left(1-P_{i}\right)}\right]
\end{aligned}
$$


com $P_{i}^{*}$ e $P_{i}$ definidos, respectivamente, em 3.2 e 3.1 em que $\theta_{j}$ é substituído por $\theta$.

Substituindo 4.12) e 4.13 em 4.11), obtém-se

$$
\begin{aligned}
& \frac{\partial l(\boldsymbol{\zeta} \mid \boldsymbol{\eta})}{\partial \boldsymbol{\zeta}_{i}}=\sum_{j=1}^{n} \frac{1}{P\left(\boldsymbol{X}_{\cdot j}=\boldsymbol{x}_{\cdot j}, \boldsymbol{C}_{\cdot j}=\boldsymbol{c}_{\cdot j} \mid \boldsymbol{\zeta}, \boldsymbol{\eta}\right)} \int_{\mathbb{R}}\left[\frac{\partial P_{i}^{*}}{\partial \boldsymbol{\zeta}_{i}} \frac{c_{i j}\left(x_{i j}-P_{i}^{*}\right)}{P_{i}^{*}\left(1-P_{i}^{*}\right)}+\frac{\partial \delta_{i}}{\partial \boldsymbol{\zeta}_{i}} \frac{\left(c_{i j}-\delta_{i}\right)}{\delta_{i}\left(1-\delta_{i}\right)}+\right. \\
& \left.\frac{\partial P_{i}}{\partial \boldsymbol{\zeta}_{i}} \frac{\left(1-c_{i j}\right)\left(x_{i j}-P_{i}\right)}{P_{i}\left(1-P_{i}\right)}\right] P\left(\boldsymbol{X}_{\cdot j}=\boldsymbol{x}_{\cdot j}, \boldsymbol{C}_{\cdot j}=\boldsymbol{c}_{\cdot j} \mid \theta, \boldsymbol{\zeta}\right) g(\theta \mid \boldsymbol{\eta}) d \theta \\
& =\sum_{j=1}^{n} \int_{\mathbb{R}}\left[\frac{\partial P_{i}^{*}}{\partial \boldsymbol{\zeta}_{i}} \frac{c_{i j}\left(x_{i j}-P_{i}^{*}\right)}{P_{i}^{*}\left(1-P_{i}^{*}\right)}+\frac{\partial \delta_{i}}{\partial \boldsymbol{\zeta}_{i}} \frac{\left(c_{i j}-\delta_{i}\right)}{\delta_{i}\left(1-\delta_{i}\right)}+\frac{\partial P_{i}}{\partial \boldsymbol{\zeta}_{i}} \frac{\left(1-c_{i j}\right)\left(x_{i j}-P_{i}\right)}{P_{i}\left(1-P_{i}\right)}\right] g_{j}^{*}(\theta \mid \boldsymbol{\zeta}, \boldsymbol{\eta}) d \theta
\end{aligned}
$$

com

$$
g_{j}^{*}(\theta \mid \boldsymbol{\zeta}, \boldsymbol{\eta})=\frac{P\left(\boldsymbol{X}_{\cdot j}=\boldsymbol{x}_{\cdot j}, \boldsymbol{C}_{\cdot j}=\boldsymbol{c}_{\cdot j} \mid \theta, \boldsymbol{\zeta}\right) g(\theta \mid \boldsymbol{\eta})}{P\left(\boldsymbol{X}_{\cdot j}=\boldsymbol{x}_{\cdot j}, \boldsymbol{C}_{\cdot j}=\boldsymbol{c}_{\cdot j} \mid \boldsymbol{\zeta}, \boldsymbol{\eta}\right)}
$$

As derivadas parciais de $l(\boldsymbol{\zeta} \mid \boldsymbol{\eta})$ com respeito a cada um dos parâmetros de item são dadas pela substituição das expressões indicadas em (A.8)-A.16 em 4.14). As equações de estimação, já simplificadas, são:

$$
\begin{aligned}
& \frac{\partial l(\boldsymbol{\zeta} \mid \boldsymbol{\eta})}{\partial a_{i}}=\sum_{j=1}^{n} \int_{\mathbb{R}}\left[\frac{c_{i j}\left(x_{i j}-P_{i}^{*}\right)\left(\gamma_{i}-P_{i}^{*}\right)\left(\theta-b_{2 i}\right)}{\gamma_{i}\left(1-P_{i}^{*}\right)}+\right. \\
& \left.+\left(1-c_{i j}\right)\left(x_{i j}-P_{i}\right)\left(\theta-b_{1 i}\right)\right] g_{j}^{*}(\theta \mid \boldsymbol{\zeta}, \boldsymbol{\eta}) d \theta=0, \\
& \frac{\partial l(\boldsymbol{\zeta} \mid \boldsymbol{\eta})}{\partial b_{1 i}}=\sum_{j=1}^{n} \int_{\mathbb{R}}\left[-a_{i}\left(1-c_{i j}\right)\left(x_{i j}-P_{i}\right)\right] g_{j}^{*}(\theta \mid \boldsymbol{\zeta}, \boldsymbol{\eta}) d \theta=0, \\
& \frac{\partial l(\boldsymbol{\zeta} \mid \boldsymbol{\eta})}{\partial b_{2 i}}=\sum_{j=1}^{n} \int_{\mathbb{R}}\left[\frac{c_{i j}\left(x_{i j}-P_{i}^{*}\right)\left(\gamma_{i}-P_{i}^{*}\right)\left(-a_{i}\right)}{\gamma_{i}\left(1-P_{i}^{*}\right)}\right] g_{j}^{*}(\theta \mid \boldsymbol{\zeta}, \boldsymbol{\eta}) d \theta=0 \\
& \frac{\partial l(\boldsymbol{\zeta} \mid \boldsymbol{\eta})}{\partial \gamma_{i}}=\sum_{j=1}^{n} \int_{\mathbb{R}}\left[\frac{c_{i j}\left(x_{i j}-P_{i}^{*}\right)}{\gamma_{i}\left(1-P_{i}^{*}\right)}\right] g_{j}^{*}(\theta \mid \boldsymbol{\zeta}, \boldsymbol{\eta}) d \theta=0,
\end{aligned}
$$




$$
\begin{aligned}
\frac{\partial l(\boldsymbol{\zeta} \mid \boldsymbol{\eta})}{\partial \delta_{i}} & =\sum_{j=1}^{n} \frac{\left(c_{i j}-\delta_{i}\right)}{\delta_{i}\left(1-\delta_{i}\right)} \int_{\mathbb{R}} g_{j}^{*}(\theta \mid \boldsymbol{\zeta}, \boldsymbol{\eta}) d \theta \\
& =\sum_{j=1}^{n} \frac{\left(c_{i j}-\delta_{i}\right)}{\delta_{i}\left(1-\delta_{i}\right)}=0 .
\end{aligned}
$$

A equação 4.20 é a única que apresenta solução explícita, dada por $\hat{\delta}_{i}=\sum_{j=1}^{n} c_{i j} / n$. As equações (4.16)-(4.19) necessitam de algum método iterativo para serem resolvidas. Para a utilização dos métodos de Newton-Raphson ou Scoring de Fisher, é necessário calcular

$$
\begin{aligned}
& \frac{\partial^{2} l(\boldsymbol{\zeta} \mid \boldsymbol{\eta})}{\partial \boldsymbol{\zeta}_{l} \partial \boldsymbol{\zeta}_{i}^{t}}=\frac{\partial}{\partial \boldsymbol{\zeta}_{l}}\left[\frac{\partial l(\boldsymbol{\zeta} \mid \boldsymbol{\eta})}{\partial \boldsymbol{\zeta}_{i}}\right]^{t} \\
& =\frac{\partial}{\partial \boldsymbol{\zeta}_{l}}\left[\sum_{j=1}^{n} \frac{1}{P\left(\boldsymbol{x}_{\cdot j}, \boldsymbol{c}_{\cdot j} \mid \boldsymbol{\zeta}, \boldsymbol{\eta}\right)} \frac{\partial P\left(\boldsymbol{x}_{\cdot j}, \boldsymbol{c}_{\cdot j} \mid \boldsymbol{\zeta}, \boldsymbol{\eta}\right)}{\partial \boldsymbol{\zeta}_{i}}\right]^{t} \\
& =\sum_{j=1}^{n}\left[\frac{\partial^{2} P\left(\boldsymbol{x}_{\cdot j}, \boldsymbol{c}_{\cdot j} \mid \boldsymbol{\zeta}, \boldsymbol{\eta}\right) /\left(\partial \boldsymbol{\zeta}_{l} \partial \boldsymbol{\zeta}_{i}^{t}\right)}{P\left(\boldsymbol{x}_{\cdot j}, \boldsymbol{c}_{\cdot j} \mid \boldsymbol{\zeta}, \boldsymbol{\eta}\right)}-\left(\frac{\partial P\left(\boldsymbol{x}_{\cdot j}, \boldsymbol{c}_{\cdot j} \mid \boldsymbol{\zeta}, \boldsymbol{\eta}\right) / \partial \boldsymbol{\zeta}_{l}}{P\left(\boldsymbol{x}_{\cdot j}, \boldsymbol{c}_{\cdot j} \mid \boldsymbol{\zeta}, \boldsymbol{\eta}\right)}\right)\left(\frac{\partial P\left(\boldsymbol{x}_{\cdot j}, \boldsymbol{c}_{\cdot j} \mid \boldsymbol{\zeta}, \boldsymbol{\eta}\right) / \partial \boldsymbol{\zeta}_{i}}{P\left(\boldsymbol{x}_{\cdot j}, \boldsymbol{c}_{\cdot j} \mid \boldsymbol{\zeta}, \boldsymbol{\eta}\right)}\right)^{t}\right],
\end{aligned}
$$

para $\mathrm{i}, \mathrm{l}=1, \ldots, \mathrm{I}$.

A segunda parcela da expressão entre colchetes em (4.21) pode ser escrita como $\boldsymbol{h}_{l(j)} \boldsymbol{h}_{i(j)}^{t}$, em que

$$
\begin{aligned}
\boldsymbol{h}_{i(j)} & =\frac{\partial P\left(\boldsymbol{x}_{\cdot j}, \boldsymbol{c}_{\cdot j} \mid \boldsymbol{\zeta}, \boldsymbol{\eta}\right) / \partial \boldsymbol{\zeta}_{i}}{P\left(\boldsymbol{x}_{\cdot j}, \boldsymbol{c}_{\cdot j} \mid \boldsymbol{\zeta}, \boldsymbol{\eta}\right)} \\
\quad= & \int_{\mathbb{R}}\left[\frac{\partial P_{i}^{*}}{\partial \boldsymbol{\zeta}_{i}} \frac{c_{i j}\left(x_{i j}-P_{i}^{*}\right)}{P_{i}^{*}\left(1-P_{i}^{*}\right)}+\frac{\partial \delta_{i}}{\partial \boldsymbol{\zeta}_{i}} \frac{\left(c_{i j}-\delta_{i}\right)}{\delta_{i}\left(1-\delta_{i}\right)}+\frac{\partial P_{i}}{\partial \boldsymbol{\zeta}_{i}} \frac{\left(1-c_{i j}\right)\left(x_{i j}-P_{i}\right)}{P_{i}\left(1-P_{i}\right)}\right] g_{j}^{*}(\theta \mid \boldsymbol{\zeta}, \boldsymbol{\eta}) d \theta .
\end{aligned}
$$

A última igualdade segue de 4.12, 4.13) e 4.15). Definindo:

$\boldsymbol{h}_{i}^{*}=\partial P_{i}^{*} / \partial \boldsymbol{\zeta}_{i}=\left[\begin{array}{lllll}\frac{\left(\theta-b_{2 i}\right)}{\gamma_{i}} P_{i}^{*}\left(\gamma_{i}-P_{i}^{*}\right) & 0 & \frac{-a_{i}}{\gamma_{i}} P_{i}^{*}\left(\gamma_{i}-P_{i}^{*}\right) & P_{i}^{*} / \gamma_{i} & 0\end{array}\right]^{t}$,
$\boldsymbol{h}_{i \delta}=\partial \delta_{i} / \partial \boldsymbol{\zeta}_{i}=\left[\begin{array}{lllll}0 & 0 & 0 & 0 & 1\end{array}\right]^{t} \mathrm{e}$
$\boldsymbol{h}_{i}=\partial P_{i} / \partial \boldsymbol{\zeta}_{i}=\left[\begin{array}{lllll}\left(\theta-b_{1 i}\right) P_{i}\left(1-P_{i}\right) & -a_{i} P_{i}\left(1-P_{i}\right) & 0 & 0 & 0\end{array}\right]^{t}$, pode-se reescrever 4.22 como

$$
\boldsymbol{h}_{i(j)}=\int_{\mathbb{R}}\left[\boldsymbol{h}_{i}^{*} \frac{c_{i j}\left(x_{i j}-P_{i}^{*}\right)}{P_{i}^{*}\left(1-P_{i}^{*}\right)}+\boldsymbol{h}_{i \delta} \frac{\left(c_{i j}-\delta_{i}\right)}{\delta_{i}\left(1-\delta_{i}\right)}+\boldsymbol{h}_{i} \frac{\left(1-c_{i j}\right)\left(x_{i j}-P_{i}\right)}{P_{i}\left(1-P_{i}\right)}\right] g_{j}^{*}(\theta \mid \boldsymbol{\zeta}, \boldsymbol{\eta}) d \theta
$$


Para simplificar a primeira parcela entre colchetes em (4.21), note que

$$
\begin{aligned}
& \frac{\partial^{2} P\left(\boldsymbol{x}_{\cdot j}, \boldsymbol{c}_{\cdot j} \mid \boldsymbol{\zeta}, \boldsymbol{\eta}\right)}{\left(\partial \boldsymbol{\zeta}_{i} \partial \boldsymbol{\zeta}_{i}^{t}\right)}=\frac{\partial}{\partial \boldsymbol{\zeta}_{i}}\left\{\int _ { \mathbb { R } } \left[\left(\frac{\partial P_{i}^{*}}{\partial \boldsymbol{\zeta}_{i}}\right)^{t} \frac{c_{i j}\left(x_{i j}-P_{i}^{*}\right)}{P_{i}^{*}\left(1-P_{i}^{*}\right)}+\left(\frac{\partial \delta_{i}}{\partial \boldsymbol{\zeta}_{i}}\right)^{t} \frac{\left(c_{i j}-\delta_{i}\right)}{\delta_{i}\left(1-\delta_{i}\right)}+\right.\right. \\
&\left.\left.\left(\frac{\partial P_{i}}{\partial \boldsymbol{\zeta}_{i}}\right)^{t} \frac{\left(1-c_{i j}\right)\left(x_{i j}-P_{i}\right)}{P_{i}\left(1-P_{i}\right)}\right] P\left(\boldsymbol{x}_{\cdot j}, \boldsymbol{c}_{\cdot j} \mid \theta, \boldsymbol{\zeta}\right) g(\theta \mid \boldsymbol{\eta}) d \theta\right\} \\
&=\int_{\mathbb{R}} \frac{\partial}{\partial \boldsymbol{\zeta}_{i}}\left\{\left[\left(\frac{\partial P_{i}^{*}}{\partial \boldsymbol{\zeta}_{i}}\right)^{t} \frac{c_{i j}\left(x_{i j}-P_{i}^{*}\right)}{P_{i}^{*}\left(1-P_{i}^{*}\right)}+\left(\frac{\partial \delta_{i}}{\partial \boldsymbol{\zeta}_{i}}\right)^{t} \frac{\left(c_{i j}-\delta_{i}\right)}{\delta_{i}\left(1-\delta_{i}\right)}+\right.\right. \\
&\left.\left.\left(\frac{\partial P_{i}}{\partial \boldsymbol{\zeta}_{i}}\right)^{t} \frac{\left(1-c_{i j}\right)\left(x_{i j}-P_{i}\right)}{P_{i}\left(1-P_{i}\right)}\right] P\left(\boldsymbol{x}_{\cdot j}, \boldsymbol{c}_{\cdot j} \mid \theta, \boldsymbol{\zeta}\right)\right\} g(\theta \mid \boldsymbol{\eta}) d \theta \\
&= \int_{\mathbb{R}}\left(\frac{\partial^{2} P_{i}^{*}}{\partial \boldsymbol{\zeta}_{i} \partial \boldsymbol{\zeta}_{i}^{t}} \frac{c_{i j}\left(x_{i j}-P_{i}^{*}\right)}{P_{i}^{*}\left(1-P_{i}^{*}\right)}+\frac{\partial^{2} P_{i}}{\partial \boldsymbol{\zeta}_{i} \partial \boldsymbol{\zeta}_{i}^{t}} \frac{\left(1-c_{i j}\right)\left(x_{i j}-P_{i}\right)}{P_{i}\left(1-P_{i}\right)}-\right. \\
&\left(\frac{\partial P_{i}^{*}}{\partial \boldsymbol{\zeta}_{i}}\right)\left(\frac{\partial P_{i}^{*}}{\partial \boldsymbol{\zeta}_{i}}\right)^{t} c_{i j}\left(1-c_{i j}\right)\left[\frac{\left(x_{i j}-P_{i}^{*}\right)}{P_{i}^{*}\left(1-P_{i}^{*}\right)}\right]^{2}- \\
&\left(\frac{\partial P_{i}}{\partial \boldsymbol{\zeta}_{i}}\right)^{2}\left(\frac{\partial P_{i}}{\partial \boldsymbol{\zeta}_{i}}\right)^{t} c_{i j}\left(1-c_{i j}\right)\left(\frac{\left(x_{i j}-P_{i}\right)}{P_{i}\left(1-P_{i}\right)}\right)^{2}+ \\
& {\left[\left(\frac{\partial P_{i}^{*}}{\partial \boldsymbol{\zeta}_{i}}\right)\left(\frac{\partial \delta_{i}}{\partial \boldsymbol{\zeta}_{i}}\right)^{t}+\left(\frac{\partial \delta_{i}}{\partial \boldsymbol{\zeta}_{i}}\right)\left(\frac{\partial P_{i}^{*}}{\partial \boldsymbol{\zeta}_{i}}\right)^{t}\right] \frac{c_{i j}\left(x_{i j}-P_{i}^{*}\right)}{P_{i}^{*}\left(1-P_{i}^{*}\right)} \frac{\left(c_{i j}-\delta_{i}\right)}{\delta_{i}\left(1-\delta_{i}\right)}+} \\
& {\left[\left(\frac{\partial P_{i}^{*}}{\partial \boldsymbol{\zeta}_{i}}\right)\left(\frac{\partial P_{i}}{\partial \boldsymbol{\zeta}_{i}}\right)^{t}+\left(\frac{\partial P_{i}}{\partial \boldsymbol{\zeta}_{i}}\right)\left(\frac{\partial P_{i}^{*}}{\partial \boldsymbol{\zeta}_{i}}\right)^{t}\right] \frac{\left(1-c_{i j}\right)\left(x_{i j}-P_{i}\right)}{P_{i}\left(1-P_{i}\right)} \frac{c_{i j}\left(x_{i j}-P_{i}^{*}\right)}{P_{i}^{*}\left(1-P_{i}^{*}\right)}+} \\
& {\left.\left[\left(\frac{\partial P_{i}}{\partial \boldsymbol{\zeta}_{i}}\right)\left(\frac{\partial \delta_{i}}{\partial \boldsymbol{\zeta}_{i}}\right)^{t}+\left(\frac{\partial \delta_{i}}{\partial \boldsymbol{\zeta}_{i}}\right)\left(\frac{\partial P_{i}}{\partial \boldsymbol{\zeta}_{i}}\right)^{t}\right] \frac{\left(1-c_{i j}\right)\left(x_{i j}-P_{i}\right)}{P_{i}\left(1-P_{i}\right)} \frac{\left(c_{i j}-\delta_{i}\right)}{\delta_{i}\left(1-\delta_{i}\right)}\right\} } \\
& P\left(\boldsymbol{x} \cdot j, \boldsymbol{c}_{\cdot j} \mid \theta, \boldsymbol{\zeta}\right) g(\theta \mid \boldsymbol{\eta}) d \theta .
\end{aligned}
$$

A terceira igualdade acima segue da expressão detalhada em (A.20). 
Então:

$$
\begin{aligned}
\boldsymbol{H}_{i i(j)}= & \frac{\partial^{2} P\left(\boldsymbol{x}_{\cdot j}, \boldsymbol{c}_{\cdot j} \mid \boldsymbol{\zeta}, \boldsymbol{\eta}\right) / \partial \boldsymbol{\zeta}_{i} \partial \boldsymbol{\zeta}_{i}^{t}}{P\left(\boldsymbol{x}_{\cdot j}, \boldsymbol{c}_{\cdot j} \mid \boldsymbol{\zeta}, \boldsymbol{\eta}\right)} \\
= & \int_{\mathbb{R}}\left\{\frac{\partial^{2} P_{i}^{*}}{\partial \boldsymbol{\zeta}_{i} \partial \boldsymbol{\zeta}_{i}^{t}} \frac{c_{i j}\left(x_{i j}-P_{i}^{*}\right)}{P_{i}^{*}\left(1-P_{i}^{*}\right)}+\frac{\partial^{2} P_{i}}{\partial \boldsymbol{\zeta}_{i} \partial \boldsymbol{\zeta}_{i}^{t}} \frac{\left(1-c_{i j}\right)\left(x_{i j}-P_{i}\right)}{P_{i}\left(1-P_{i}\right)}-\right. \\
& \left(\frac{\partial P_{i}^{*}}{\partial \boldsymbol{\zeta}_{i}}\right)\left(\frac{\partial P_{i}^{*}}{\partial \boldsymbol{\zeta}_{i}}\right)^{t} c_{i j}\left(1-c_{i j}\right)\left[\frac{\left(x_{i j}-P_{i}^{*}\right)}{P_{i}^{*}\left(1-P_{i}^{*}\right)}\right]^{2}- \\
& \left(\frac{\partial P_{i}}{\partial \boldsymbol{\zeta}_{i}}\right)\left(\frac{\partial P_{i}}{\partial \boldsymbol{\zeta}_{i}}\right)^{t} c_{i j}\left(1-c_{i j}\right)\left(\frac{\left(x_{i j}-P_{i}\right)}{P_{i}\left(1-P_{i}\right)}\right)^{2}+ \\
& {\left[\left(\frac{\partial P_{i}^{*}}{\partial \boldsymbol{\zeta}_{i}}\right)\left(\frac{\partial \delta_{i}}{\partial \boldsymbol{\zeta}_{i}}\right)^{t}+\left(\frac{\partial \delta_{i}}{\partial \boldsymbol{\zeta}_{i}}\right)\left(\frac{\partial P_{i}^{*}}{\partial \boldsymbol{\zeta}_{i}}\right)^{t}\right] \frac{c_{i j}\left(x_{i j}-P_{i}^{*}\right)}{P_{i}^{*}\left(1-P_{i}^{*}\right)} \frac{\left(c_{i j}-\delta_{i}\right)}{\delta_{i}\left(1-\delta_{i}\right)}+} \\
& {\left[\left(\frac{\partial P_{i}^{*}}{\partial \boldsymbol{\zeta}_{i}}\right)\left(\frac{\partial P_{i}}{\partial \boldsymbol{\zeta}_{i}}\right)^{t}+\left(\frac{\partial P_{i}}{\partial \boldsymbol{\zeta}_{i}}\right)\left(\frac{\partial P_{i}^{*}}{\partial \boldsymbol{\zeta}_{i}}\right)^{t}\right] \frac{\left(1-c_{i j}\right)\left(x_{i j}-P_{i}\right)}{P_{i}\left(1-P_{i}\right)} \frac{c_{i j}\left(x_{i j}-P_{i}^{*}\right)}{P_{i}^{*}\left(1-P_{i}^{*}\right)}+} \\
& {\left.\left[\left(\frac{\partial P_{i}}{\partial \boldsymbol{\zeta}_{i}}\right)\left(\frac{\partial \delta_{i}}{\partial \boldsymbol{\zeta}_{i}}\right)^{t}+\left(\frac{\partial \delta_{i}}{\partial \boldsymbol{\zeta}_{i}}\right)\left(\frac{\partial P_{i}}{\partial \boldsymbol{\zeta}_{i}}\right)^{t}\right] \frac{\left(1-c_{i j}\right)\left(x_{i j}-P_{i}\right)}{P_{i}\left(1-P_{i}\right)} \frac{\left(c_{i j}-\delta_{i}\right)}{\delta_{i}\left(1-\delta_{i}\right)}\right\}_{j}^{*}(\theta \mid \boldsymbol{\zeta}, \boldsymbol{\eta}) d \theta . }
\end{aligned}
$$

Tendo em conta as expressões detalhadas em A.21 - A.28 e A.30 -A.33, pode-se escrever

$$
\begin{aligned}
& \boldsymbol{H}_{i i}^{*}=\frac{\partial^{2} P_{i}^{*}}{\partial \boldsymbol{\zeta}_{i} \partial \boldsymbol{\zeta}_{i}^{t}}
\end{aligned}
$$

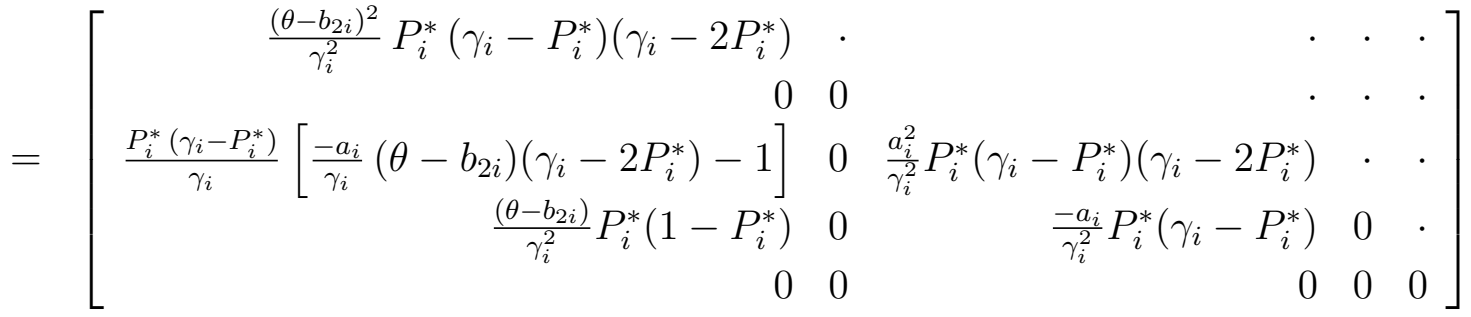

e 


$$
\begin{aligned}
& \boldsymbol{H}_{i i}=\frac{\partial^{2} P_{i}}{\partial \boldsymbol{\zeta}_{i} \partial \boldsymbol{\zeta}_{i}^{t}} \\
& =\left[\begin{array}{rrccc}
\left(\theta-b_{1 i}\right)^{2} P_{i}\left(1-P_{i}\right)\left(1-2 P_{i}\right) & . & . & . & . \\
-P_{i}\left(1-P_{i}\right)\left[1+a_{i}\left(\theta-b_{1 i}\right)\left(1-2 P_{i}\right)\right] & a_{i}^{2} P_{i}\left(1-P_{i}\right)\left(1-2 P_{i}\right) & . & . & . \\
0 & 0 & 0 & . & . \\
0 & 0 & 0 & 0 & . \\
0 & 0 & 0 & 0 & 0
\end{array}\right] .
\end{aligned}
$$

Portanto:

$$
\begin{aligned}
\boldsymbol{H}_{i i(j)}= & \int_{\mathbb{R}}\left\{\frac{c_{i j}\left(x_{i j}-P_{i}^{*}\right)}{P_{i}^{*}\left(1-P_{i}^{*}\right)} \boldsymbol{H}_{i i}^{*}+\frac{\left(1-c_{i j}\right)\left(x_{i j}-P_{i}\right)}{P_{i}\left(1-P_{i}\right)} \boldsymbol{H}_{i i}+\right. \\
& {\left[\boldsymbol{h}_{i}^{*} \boldsymbol{h}_{i \delta}^{t}+\boldsymbol{h}_{i \delta}\left(\boldsymbol{h}_{i}^{*}\right)^{t}\right] \frac{c_{i j}\left(x_{i j}-P_{i}^{*}\right)}{P_{i}^{*}\left(1-P_{i}^{*}\right)} \frac{c_{i j}-\delta_{i}}{\delta_{i}\left(1-\delta_{i}\right)}+} \\
& {\left[\boldsymbol{h}_{i}^{*} \boldsymbol{h}_{i}^{t}+\boldsymbol{h}_{i}\left(\boldsymbol{h}_{i}^{*}\right)^{t}\right] \frac{c_{i j}\left(x_{i j}-P_{i}^{*}\right)}{P_{i}^{*}\left(1-P_{i}^{*}\right)} \frac{\left(1-c_{i j}\right)\left(x_{i j}-P_{i}\right)}{P_{i}\left(1-P_{i}\right)}+} \\
& {\left.\left[\boldsymbol{h}_{i} \boldsymbol{h}_{i \delta}^{t}+\boldsymbol{h}_{i \delta}\left(\boldsymbol{h}_{i}\right)^{t}\right] \frac{c_{i j}-\delta_{i}}{\delta_{i}\left(1-\delta_{i}\right)} \frac{\left(1-c_{i j}\right)\left(x_{i j}-P_{i}\right)}{P_{i}\left(1-P_{i}\right)}\right\} g_{j}^{*}(\theta \mid \boldsymbol{\zeta}, \boldsymbol{\eta}) d \theta . }
\end{aligned}
$$

Por outro lado, observe que

$$
\begin{aligned}
& \frac{\partial^{2} P\left(\boldsymbol{x}_{\cdot j}, \boldsymbol{c}_{\cdot j} \mid \boldsymbol{\zeta}, \boldsymbol{\eta}\right)}{\left(\partial \boldsymbol{\zeta}_{l} \partial \boldsymbol{\zeta}_{i}^{t}\right)}=\frac{\partial}{\partial \boldsymbol{\zeta}_{l}}\left\{\int _ { \mathbb { R } } \left[\left(\frac{\partial P_{i}^{*}}{\partial \boldsymbol{\zeta}_{i}}\right)^{t} \frac{c_{i j}\left(x_{i j}-P_{i}^{*}\right)}{P_{i}^{*}\left(1-P_{i}^{*}\right)}+\left(\frac{\partial \delta_{i}}{\partial \boldsymbol{\zeta}_{i}}\right)^{t} \frac{\left(c_{i j}-\delta_{i}\right)}{\delta_{i}\left(1-\delta_{i}\right)}+\right.\right. \\
&\left.\left.\left(\frac{\partial P_{i}}{\partial \boldsymbol{\zeta}_{i}}\right)^{t} \frac{\left(1-c_{i j}\right)\left(x_{i j}-P_{i}\right)}{P_{i}\left(1-P_{i}\right)}\right] P\left(\boldsymbol{x}_{\cdot j}, \boldsymbol{c}_{\cdot j} \mid \theta, \boldsymbol{\zeta}\right) g(\theta \mid \boldsymbol{\eta}) d \theta\right\} \\
&=\quad \int_{\mathbb{R}}\left(\frac{\partial P\left(\boldsymbol{x}_{\cdot j}, \boldsymbol{c}_{\cdot j} \mid \theta, \boldsymbol{\zeta}\right)}{\partial \boldsymbol{\zeta}_{l}}\right)\left[\left(\frac{\partial P_{i}^{*}}{\partial \boldsymbol{\zeta}_{i}}\right)^{t} \frac{c_{i j}\left(x_{i j}-P_{i}^{*}\right)}{P_{i}^{*}\left(1-P_{i}^{*}\right)}+\left(\frac{\partial \delta_{i}}{\partial \boldsymbol{\zeta}_{i}}\right)^{t} \frac{\left(c_{i j}-\delta_{i}\right)}{\delta_{i}\left(1-\delta_{i}\right)}+\right. \\
&\left.\left(\frac{\partial P_{i}}{\partial \boldsymbol{\zeta}_{i}}\right)^{t} \frac{\left(1-c_{i j}\right)\left(x_{i j}-P_{i}\right)}{P_{i}\left(1-P_{i}\right)}\right] g(\theta \mid \boldsymbol{\eta}) d \theta \\
&= \int_{\mathbb{R}}\left[\left(\frac{\partial P_{l}^{*}}{\partial \boldsymbol{\zeta}_{l}}\right) \frac{c_{l j}\left(x_{l j}-P_{l}^{*}\right)}{P_{l}^{*}\left(1-P_{l}^{*}\right)}+\left(\frac{\partial \delta_{l}}{\partial \boldsymbol{\zeta}_{l}}\right) \frac{\left(c_{l j}-\delta_{l}\right)}{\delta_{l}\left(1-\delta_{l}\right)}+\left(\frac{\partial P_{l}}{\partial \boldsymbol{\zeta}_{l}}\right) \frac{\left(1-c_{l j}\right)\left(x_{l j}-P_{l}\right)}{P_{l}\left(1-P_{l}\right)}\right] \\
& P\left(\boldsymbol{x}_{\cdot j}, \boldsymbol{c}_{\cdot j} \mid \theta, \boldsymbol{\zeta}\right)\left[\left(\frac{\partial P_{i}^{*}}{\partial \boldsymbol{\zeta}_{i}}\right)^{t} \frac{c_{i j}\left(x_{i j}-P_{i}^{*}\right)}{P_{i}^{*}\left(1-P_{i}^{*}\right)}+\left(\frac{\partial \delta_{i}}{\partial \boldsymbol{\zeta}_{i}}\right)^{t} \frac{\left(c_{i j}-\delta_{i}\right)}{\delta_{i}\left(1-\delta_{i}\right)}+\right. \\
&\left.\left(\frac{\partial P_{i}}{\partial \boldsymbol{\zeta}_{i}}\right)^{t} \frac{\left(1-c_{i j}\right)\left(x_{i j}-P_{i}\right)}{P_{i}\left(1-P_{i}\right)}\right] g(\theta \mid \boldsymbol{\eta}) d \theta .
\end{aligned}
$$


Portanto

$$
\begin{aligned}
& \boldsymbol{H}_{i l(j)}=\frac{\partial^{2} P\left(\boldsymbol{x}_{\cdot j}, \boldsymbol{c}_{\cdot j} \mid \boldsymbol{\zeta}, \boldsymbol{\eta}\right) / \partial \boldsymbol{\zeta}_{l} \boldsymbol{\zeta}_{i}^{t}}{P\left(\boldsymbol{x}_{\cdot j}, \boldsymbol{c}_{\cdot j} \mid \boldsymbol{\zeta}, \boldsymbol{\eta}\right)} \\
& =\int_{\mathbb{R}}\left\{\left(\frac{\partial P_{l}^{*}}{\partial \boldsymbol{\zeta}_{l}}\right)\left(\frac{\partial P_{i}^{*}}{\partial \boldsymbol{\zeta}_{i}}\right)^{t} \frac{c_{l j}\left(x_{l j}-P_{l}^{*}\right)}{P_{l}^{*}\left(1-P_{l}^{*}\right)} \frac{c_{i j}\left(x_{i j}-P_{i}^{*}\right)}{P_{i}^{*}\left(1-P_{i}^{*}\right)}+\right. \\
& \left(\frac{\partial P_{l}^{*}}{\partial \boldsymbol{\zeta}_{l}}\right)\left(\frac{\partial \delta_{i}}{\partial \boldsymbol{\zeta}_{i}}\right)^{t} \frac{c_{l j}\left(x_{l j}-P_{l}^{*}\right)}{P_{l}^{*}\left(1-P_{l}^{*}\right)} \frac{\left(c_{i j}-\delta_{i}\right)}{\delta_{i}\left(1-\delta_{i}\right)}+ \\
& \left(\frac{\partial P_{l}^{*}}{\partial \boldsymbol{\zeta}_{l}}\right)\left(\frac{\partial P_{i}}{\partial \boldsymbol{\zeta}_{i}}\right)^{t} \frac{c_{l j}\left(x_{l j}-P_{l}^{*}\right)}{P_{l}^{*}\left(1-P_{l}^{*}\right)} \frac{\left(1-c_{i j}\right)\left(x_{i j}-P_{i}\right)}{P_{i}\left(1-P_{i}\right)}+ \\
& \left(\frac{\partial \delta_{l}}{\partial \boldsymbol{\zeta}_{l}}\right)\left(\frac{\partial P_{i}^{*}}{\partial \boldsymbol{\zeta}_{i}}\right)^{t} \frac{\left(c_{l j}-\delta_{l}\right)}{\delta_{l}\left(1-\delta_{l}\right)} \frac{c_{i j}\left(x_{i j}-P_{i}^{*}\right)}{P_{i}^{*}\left(1-P_{i}^{*}\right)}+ \\
& \left(\frac{\partial \delta_{l}}{\partial \boldsymbol{\zeta}_{l}}\right)\left(\frac{\partial \delta_{i}}{\partial \boldsymbol{\zeta}_{i}}\right)^{t} \frac{\left(c_{l j}-\delta_{l}\right)}{\delta_{l}\left(1-\delta_{l}\right)} \frac{\left(c_{i j}-\delta_{i}\right)}{\delta_{i}\left(1-\delta_{i}\right)}+ \\
& \left(\frac{\partial \delta_{l}}{\partial \boldsymbol{\zeta}_{l}}\right)\left(\frac{\partial P_{i}}{\partial \boldsymbol{\zeta}_{i}}\right)^{t} \frac{\left(c_{l j}-\delta_{l}\right)}{\delta_{l}\left(1-\delta_{l}\right)} \frac{\left(1-c_{i j}\right)\left(x_{i j}-P_{i}\right)}{P_{i}\left(1-P_{i}\right)}+ \\
& \left(\frac{\partial P_{l}}{\partial \boldsymbol{\zeta}_{l}}\right)\left(\frac{\partial P_{i}^{*}}{\partial \boldsymbol{\zeta}_{i}}\right)^{t} \frac{\left(1-c_{l j}\right)\left(x_{l j}-P_{l}\right)}{P_{l}\left(1-P_{l}\right)} \frac{c_{i j}\left(x_{i j}-P_{i}^{*}\right)}{P_{i}^{*}\left(1-P_{i}^{*}\right)}+ \\
& \left(\frac{\partial P_{l}}{\partial \boldsymbol{\zeta}_{l}}\right)\left(\frac{\partial \delta_{i}}{\partial \boldsymbol{\zeta}_{i}}\right)^{t} \frac{\left(1-c_{l j}\right)\left(x_{l j}-P_{l}\right)}{P_{l}\left(1-P_{l}\right)} \frac{\left(c_{i j}-\delta_{i}\right)}{\delta_{i}\left(1-\delta_{i}\right)}+ \\
& \left.\left(\frac{\partial P_{l}}{\partial \boldsymbol{\zeta}_{l}}\right)\left(\frac{\partial P_{i}}{\partial \boldsymbol{\zeta}_{i}}\right)^{t} \frac{\left(1-c_{l j}\right)\left(x_{l j}-P_{l}\right)}{P_{l}\left(1-P_{l}\right)} \frac{\left(1-c_{i j}\right)\left(x_{i j}-P_{i}\right)}{P_{i}\left(1-P_{i}\right)}\right\} g_{j}^{*}(\theta \mid \boldsymbol{\zeta}, \boldsymbol{\eta}) d \theta \\
& =\int_{\mathbb{R}}\left\{\boldsymbol{h}_{l}^{*}\left(\boldsymbol{h}_{i}^{*}\right)^{t} \frac{c_{l j}\left(x_{l j}-P_{l}^{*}\right)}{P_{l}^{*}\left(1-P_{l}^{*}\right)} \frac{c_{i j}\left(x_{i j}-P_{i}^{*}\right)}{P_{i}^{*}\left(1-P_{i}^{*}\right)}+\boldsymbol{h}_{l}^{*}\left(\boldsymbol{h}_{i \delta}\right)^{t} \frac{c_{l j}\left(x_{l j}-P_{l}^{*}\right)}{P_{l}^{*}\left(1-P_{l}^{*}\right)} \frac{\left(c_{i j}-\delta_{i}\right)}{\delta_{i}\left(1-\delta_{i}\right)}+\right. \\
& \boldsymbol{h}_{l}^{*}\left(\boldsymbol{h}_{i}\right)^{t} \frac{c_{l j}\left(x_{l j}-P_{l}^{*}\right)}{P_{l}^{*}\left(1-P_{l}^{*}\right)} \frac{\left(1-c_{i j}\right)\left(x_{i j}-P_{i}\right)}{P_{i}\left(1-P_{i}\right)}+\boldsymbol{h}_{l \delta}\left(\boldsymbol{h}_{i}^{*}\right)^{t} \frac{\left(c_{l j}-\delta_{l}\right)}{\delta_{l}\left(1-\delta_{l}\right)} \frac{c_{i j}\left(x_{i j}-P_{i}^{*}\right)}{P_{i}^{*}\left(1-P_{i}^{*}\right)}+ \\
& \boldsymbol{h}_{l \delta}\left(\boldsymbol{h}_{i \delta}\right)^{t} \frac{\left(c_{l j}-\delta_{l}\right)}{\delta_{l}\left(1-\delta_{l}\right)} \frac{\left(c_{i j}-\delta_{i}\right)}{\delta_{i}\left(1-\delta_{i}\right)}+\boldsymbol{h}_{l \delta}\left(\boldsymbol{h}_{i}\right)^{t} \frac{\left(c_{l j}-\delta_{l}\right)}{\delta_{l}\left(1-\delta_{l}\right)} \frac{\left(1-c_{i j}\right)\left(x_{i j}-P_{i}\right)}{P_{i}\left(1-P_{i}\right)}+ \\
& \boldsymbol{h}_{l}\left(\boldsymbol{h}_{i}^{*}\right)^{t} \frac{\left(1-c_{l j}\right)\left(x_{l j}-P_{l}\right)}{P_{l}\left(1-P_{l}\right)} \frac{c_{i j}\left(x_{i j}-P_{i}^{*}\right)}{P_{i}^{*}\left(1-P_{i}^{*}\right)}+\boldsymbol{h}_{l}\left(\boldsymbol{h}_{i \delta}\right)^{t} \frac{\left(1-c_{l j}\right)\left(x_{l j}-P_{l}\right)}{P_{l}\left(1-P_{l}\right)} \frac{\left(c_{i j}-\delta_{i}\right)}{\delta_{i}\left(1-\delta_{i}\right)}+ \\
& \left.\boldsymbol{h}_{l}\left(\boldsymbol{h}_{i}\right)^{t} \frac{\left(1-c_{l j}\right)\left(x_{l j}-P_{l}\right)}{P_{l}\left(1-P_{l}\right)} \frac{\left(1-c_{i j}\right)\left(x_{i j}-P_{i}\right)}{P_{i}\left(1-P_{i}\right)}\right\} g_{j}^{*}(\theta \mid \boldsymbol{\zeta}, \boldsymbol{\eta}) d \theta \text {. }
\end{aligned}
$$

Como os itens não são independentes (apesar de o serem condicionalmente a $\theta$ ), a aplicação de algum algoritmo para obtenção dos estimadores de $\zeta_{i}, i=1, \ldots, I$ deve ser 
realizada conjuntamente para todos eles. Com essa finalidade, sejam

$$
\boldsymbol{h}_{p i}(\boldsymbol{\zeta})=\left[\begin{array}{r}
\boldsymbol{h}\left(\boldsymbol{\zeta}_{1}\right) \\
\boldsymbol{h}\left(\boldsymbol{\zeta}_{2}\right) \\
\vdots \\
\boldsymbol{h}\left(\boldsymbol{\zeta}_{I}\right)
\end{array}\right] \text { e } \boldsymbol{H}_{p i}(\boldsymbol{\zeta})=\left[\begin{array}{rrrr}
\boldsymbol{H}\left(\boldsymbol{\zeta}_{1} \boldsymbol{\zeta}_{1}\right) & \boldsymbol{H}\left(\boldsymbol{\zeta}_{1} \boldsymbol{\zeta}_{2}\right) & \ldots & \boldsymbol{H}\left(\boldsymbol{\zeta}_{1} \boldsymbol{\zeta}_{I}\right) \\
\boldsymbol{H}\left(\boldsymbol{\zeta}_{2} \boldsymbol{\zeta}_{1}\right) & \boldsymbol{H}\left(\boldsymbol{\zeta}_{2} \boldsymbol{\zeta}_{2}\right) & \ldots & \boldsymbol{H}\left(\boldsymbol{\zeta}_{2} \boldsymbol{\zeta}_{I}\right) \\
\vdots & \vdots & & \vdots \\
\boldsymbol{H}\left(\boldsymbol{\zeta}_{I} \boldsymbol{\zeta}_{1}\right) & \boldsymbol{H}\left(\boldsymbol{\zeta}_{I} \boldsymbol{\zeta}_{2}\right) & \ldots & \boldsymbol{H}\left(\boldsymbol{\zeta}_{I} \boldsymbol{\zeta}_{I}\right)
\end{array}\right]
$$

com

$$
\begin{aligned}
\boldsymbol{h}\left(\boldsymbol{\zeta}_{i}\right) & =\frac{\partial l(\boldsymbol{\zeta}, \boldsymbol{\eta})}{\partial \boldsymbol{\zeta}_{i}} \\
& =\sum_{j=1}^{n}\left[\boldsymbol{h}_{i}^{*} \frac{c_{i j}\left(x_{i j}-P_{i}^{*}\right)}{P_{i}^{*}\left(1-P_{i}^{*}\right)}+\boldsymbol{h}_{i \delta} \frac{\left(c_{i j}-\delta_{i}\right)}{\delta_{i}\left(1-\delta_{i}\right)}+\boldsymbol{h}_{i} \frac{\left(1-c_{i j}\right)\left(x_{i j}-P_{i}\right)}{P_{i}\left(1-P_{i}\right)}\right]
\end{aligned}
$$

e

$$
\boldsymbol{H}\left(\boldsymbol{\zeta}_{l} \boldsymbol{\zeta}_{i}\right)=\frac{\partial^{2} l(\boldsymbol{\zeta}, \boldsymbol{\eta})}{\partial \boldsymbol{\zeta}_{l} \partial \boldsymbol{\zeta}_{i}^{t}}=\sum_{j=1}^{n}\left[\boldsymbol{H}_{i l(j)}-\boldsymbol{h}_{l(j)} \boldsymbol{h}_{i(j)}^{t}\right]
$$

A obtenção dos estimadores e dos respectivos erros-padrão por meio do algoritmo Scoring de Fisher depende da expressão da esperança da segunda derivada da função logverossimilhança, que é dada por

$$
\begin{aligned}
E\left[\boldsymbol{H}\left(\boldsymbol{\zeta}_{l} \boldsymbol{\zeta}_{i}\right)\right] & =E\left[\sum_{j=1}^{n}\left[\boldsymbol{H}_{i l(j)}-\boldsymbol{h}_{l(j)} \boldsymbol{h}_{i(j)}^{t}\right]\right] \\
& =\sum_{j=1}^{n}\left\{E\left[\boldsymbol{H}_{i l(j)}\right]-E\left[\boldsymbol{h}_{l(j)} \boldsymbol{h}_{i(j)}^{t}\right]\right\} \\
& =-\sum_{j=1}^{n} \boldsymbol{h}_{l(j)} \boldsymbol{h}_{i(j)}^{t} .
\end{aligned}
$$

A última igualdade segue do fato de $E\left[\boldsymbol{H}\left(\boldsymbol{\zeta}_{l} \boldsymbol{\zeta}_{i}\right)\right]=E\left[E\left[\boldsymbol{H}\left(\boldsymbol{\zeta}_{l} \boldsymbol{\zeta}_{i}\right) \mid \theta\right]\right]$ e das expressões derivadas em A.34-A.37.

Como todos os parâmetros de itens devem serem estimados conjuntamente, o esforço computacional é bastante grande, tornando essa metodologia inaplicável para instrumentos com mais de 12 itens. Seguindo Bock \& Aitkin (1981) para simplificar a estimação dos parâmetros no ML3, a estratégia descrita na sub-seção seguinte pode ser utilizada para estimar os parâmetros de cada item isoladamente. 


\subsubsection{Estimação de $\zeta_{i}$ para os itens individualmente}

A proposta de Bock \& Aitkin (1981) consiste numa re-estruturação das equações de estimação 4.16)- 4.19 e no uso de uma adaptação do algoritmo EM. Com essa finalidade, a partir de 4.16 tem-se

$$
\begin{aligned}
\frac{\partial l(\boldsymbol{\zeta}, \boldsymbol{\eta})}{\partial a_{i}}= & \sum_{j=1}^{n} \int_{\mathbb{R}}\left[\frac{c_{i j}\left(x_{i j}-P_{i}^{*}\right)\left(\gamma_{i}-P_{i}^{*}\right)\left(\theta-b_{2 i}\right)}{\gamma_{i}\left(1-P_{i}^{*}\right)}+\left(1-c_{i j}\right)\left(x_{i j}-P_{i}\right)\left(\theta-b_{1 i}\right)\right] g_{j}^{*}(\theta \mid \boldsymbol{\zeta}, \boldsymbol{\eta}) d \theta \\
= & \sum_{j=1}^{n} \int_{\mathbb{R}}\left\{\frac{c_{i j}\left[x_{i j} g_{j}^{*}(\theta \mid \boldsymbol{\zeta}, \boldsymbol{\eta})-P_{i}^{*} g_{j}^{*}(\theta \mid \boldsymbol{\zeta}, \boldsymbol{\eta})\right]\left(\gamma_{i}-P_{i}^{*}\right)\left(\theta-b_{2 i}\right)}{\gamma_{i}\left(1-P_{i}^{*}\right)}+\right. \\
& \left.+\left(1-c_{i j}\right)\left[x_{i j} g_{j}^{*}(\theta \mid \boldsymbol{\zeta}, \boldsymbol{\eta})-P_{i} g_{j}^{*}(\theta \mid \boldsymbol{\zeta}, \boldsymbol{\eta})\right]\left(\theta-b_{1 i}\right)\right\} d \theta \\
= & \int_{\mathbb{R}}\left\{\left(\sum_{j=1}^{n} c_{i j} x_{i j} g_{j}^{*}(\theta \mid \boldsymbol{\zeta}, \boldsymbol{\eta})-P_{i}^{*} \sum_{j=1}^{n} c_{i j} g_{j}^{*}(\theta \mid \boldsymbol{\zeta}, \boldsymbol{\eta})\right) \frac{\left(\gamma_{i}-P_{i}^{*}\right)\left(\theta-b_{2 i}\right)}{\gamma_{i}\left(1-P_{i}^{*}\right)}+\right. \\
& \left.+\left(\sum_{j=1}^{n}\left(1-c_{i j}\right) x_{i j} g_{j}^{*}(\theta \mid \boldsymbol{\zeta}, \boldsymbol{\eta})-P_{i} \sum_{j=1}^{n}\left(1-c_{i j}\right) g_{j}^{*}(\theta \mid \boldsymbol{\zeta}, \boldsymbol{\eta})\right)\left(\theta-b_{1 i}\right)\right\} d \theta .
\end{aligned}
$$

A integral da expressão acima pode ser aproximada numericamente por somas de áreas de um número finito de q retângulos, conforme o método de Hermite-Gauss (Hildebrand 1956). Os pontos médios de cada retângulo, $\bar{\theta}_{k}, \mathrm{k}=1, \ldots, \mathrm{q}$, são denominados nós ou pontos de quadratura. A cada $\bar{\theta}_{k}$ é atribuído um peso $A_{k}=A\left(\bar{\theta}_{k}\right)=g\left(\bar{\theta}_{k} \mid \boldsymbol{\eta}\right) \Delta_{k}$, em que $g\left(\bar{\theta}_{k} \mid \boldsymbol{\eta}\right)$ é o valor da função que se deseja aproximar calculada em $\bar{\theta}_{k}$ e $\Delta_{k}$ é a largura do respectivo retângulo. Então:

$$
\begin{aligned}
& \frac{\partial l(\boldsymbol{\zeta}, \boldsymbol{\eta})}{\partial a_{i}}=\sum_{k=1}^{q}\left\{\left(\sum_{j=1}^{n} c_{i j} x_{i j} g_{j k}^{*}-P_{i k}^{*} \sum_{j=1}^{n} c_{i j} g_{j k}^{*}\right) \frac{\left(\gamma_{i}-P_{i k}^{*}\right)\left(\bar{\theta}_{k}-b_{2 i}\right)}{\gamma_{i}\left(1-P_{i k}^{*}\right)}+\right. \\
& \left.\quad+\left(\sum_{j=1}^{n}\left(1-c_{i j}\right) x_{i j} g_{j k}^{*}-P_{i k} \sum_{j=1}^{n}\left(1-c_{i j}\right) g_{j k}^{*}\right)\left(\bar{\theta}_{k}-b_{1 i}\right)\right\} \\
& =\sum_{k=1}^{q}\left\{\left(\bar{r}_{2 i k}-P_{i k}^{*} \bar{f}_{2 i k}\right) \frac{\left(\gamma_{i}-P_{i k}^{*}\right)\left(\bar{\theta}_{k}-b_{2 i}\right)}{\gamma_{i}\left(1-P_{i k}^{*}\right)}+\left(\bar{r}_{1 i k}-P_{i k} \bar{f}_{1 i k}\right)\left(\bar{\theta}_{k}-b_{1 i}\right)\right\}
\end{aligned}
$$


com $P_{i k}$ e $P_{i k}^{*}$ definidos, respectivamente, em 3.1 e 3.2 em que $\theta_{j}$ é substituído por $\bar{\theta}_{k}$,

$$
\begin{aligned}
& g_{j k}^{*}=g_{j}^{*}\left(\bar{\theta}_{k} \mid \boldsymbol{\zeta}, \boldsymbol{\eta}\right) \cong \frac{P\left(\boldsymbol{X}_{\cdot j}=\boldsymbol{x}_{\cdot j}, \boldsymbol{C}_{\cdot j}=\boldsymbol{c}_{\cdot j} \mid \bar{\theta}_{k}, \boldsymbol{\zeta}\right) A_{k}}{\sum_{k=1}^{q} P\left(\boldsymbol{X}_{\cdot j}=\boldsymbol{x}_{\cdot j}, \boldsymbol{C}_{\cdot j}=\boldsymbol{c}_{\cdot j} \mid \bar{\theta}_{k}, \boldsymbol{\zeta}\right) A_{k}} \Delta_{k}^{-1} \\
& \cong \frac{P\left(\boldsymbol{X}_{\cdot j}=\boldsymbol{x}_{\cdot j}, \boldsymbol{C}_{\cdot j}=\boldsymbol{c}_{\cdot j} \mid \bar{\theta}_{k}, \boldsymbol{\zeta}\right) A_{k}}{\sum_{k=1}^{q} P\left(\boldsymbol{X}_{\cdot j}=\boldsymbol{x}_{\cdot j}, \boldsymbol{C}_{\cdot j}=\boldsymbol{c}_{\cdot j} \mid \bar{\theta}_{k}, \boldsymbol{\zeta}\right) A_{k}} \\
& \bar{r}_{1 i k}=\sum_{j=1}^{n}\left(1-c_{i j}\right) x_{i j} g_{j k}^{*}, \bar{f}_{1 i k}=\sum_{j=1}^{n}\left(1-c_{i j}\right) g_{j k}^{*}, \bar{r}_{2 i k}=\sum_{j=1}^{n} c_{i j} x_{i j} g_{j k}^{*} \text { e } \bar{f}_{2 i k}=\sum_{j=1}^{n} c_{i j} g_{j k}^{*} .
\end{aligned}
$$

Analogamente, para os demais parâmetros do modelo proposto, tem-se

$$
\begin{gathered}
\frac{\partial l(\boldsymbol{\zeta} \mid \boldsymbol{\eta})}{\partial b_{1 i}}=-a_{i} \sum_{k=1}^{q}\left(\bar{r}_{1 i k}-P_{i k} \bar{f}_{1 i k}\right), \\
\frac{\partial l(\boldsymbol{\zeta} \mid \boldsymbol{\eta})}{\partial b_{2 i}}=-a_{i} \sum_{k=1}^{q}\left\{\left(\bar{r}_{2 i k}-P_{i k}^{*} \bar{f}_{2 i k}\right) \frac{\gamma_{i}-P_{i k}^{*}}{\gamma_{i}\left(1-P_{i k}^{*}\right)}\right\}, \\
\frac{\partial l(\boldsymbol{\zeta} \mid \boldsymbol{\eta})}{\partial \gamma_{i}}=\sum_{k=1}^{q}\left\{\left(\bar{r}_{2 i k}-P_{i k}^{*} \bar{f}_{2 i k}\right) \frac{1}{\gamma_{i}\left(1-P_{i k}^{*}\right)}\right\} .
\end{gathered}
$$

\section{Adaptação do Algoritmo EM}

O objetivo é estimar os parâmetros de itens, $\boldsymbol{\zeta}$, com base nas variáveis observadas, $\boldsymbol{X}_{. .}=\left(\boldsymbol{X}_{.1}, \ldots, \boldsymbol{X}_{. n}\right)$ e $\boldsymbol{C} . .=\left(\boldsymbol{C}_{.1}, \ldots, \boldsymbol{C}_{. n}\right)$, considerando $\boldsymbol{\theta}=\left(\theta_{1}, \ldots, \theta_{n}\right)$ como variáveis não-observadas.

Com tal finalidade, discretiza-se o espaço amostral dos traços latentes $\theta_{j}$ considerando um conjunto de q valores, $\bar{\theta}_{1}, \bar{\theta}_{2}, \ldots, \bar{\theta}_{q}$, associadas às probabilidades $\pi_{1}, \pi_{2}, \ldots, \pi_{q}$, respectivamente. A aproximação das integrais pelo método de quadratura, especificando-se $\overline{\theta_{k}}$, $\mathrm{k}=1, \ldots, \mathrm{q}$, como os nós, justifica tal discretização. Seja $Z_{i j k}=1$, se o indivíduo j responde o item i e apresenta nível $\bar{\theta}_{k}$ do traço latente, e $Z_{i j k}=0$, em caso contrário, e denote por $f_{1 i k}=\sum_{j=1}^{n}\left(1-c_{i j}\right) Z_{i j k}$, o número de indivíduos não constrangidos que respondem ao item i e apresentam traço latente $\bar{\theta}_{k}$, por $r_{1 i k}=\sum_{j=1}^{n}\left(1-c_{i j}\right) Z_{i j k} x_{i j}$, o número de indivíduos não constrangidos, com traço latente $\bar{\theta}_{k}$, que apresentam o sintoma relatado no item i, por $f_{2 i k}=\sum_{j=1}^{n} c_{i j} Z_{i j k}$, o número de indivíduos constrangidos, com traço latente $\bar{\theta}_{k}$, que 
respondem ao item i, por $r_{2 i k}=\sum_{j=1}^{n} c_{i j} Z_{i j k} x_{i j}$, o número de indivíduos constrangidos, com traço latente $\bar{\theta}_{k}$, que apresentam o sintoma relatado no item i, por $\sum_{k=1}^{q} f_{1 i k}=n_{1 i}$, o número de indivíduos não constrangidos que respondem ao item i, por $\sum_{k=1}^{q} f_{2 i k}=n_{2 i}$, o número de indivíduos constrangidos que respondem ao item i; e, finalmente, por $n_{1 i}+n_{2 i}=n_{i}$, o número total de indivíduos que respondem ao item i.

Se $n_{i}$ indivíduos selecionados ao acaso da população responderem ao item i, a probabilidade conjunta de que $f_{1 i k}$ indivíduos sejam não constrangidos com traço latente $\bar{\theta}_{k} \mathrm{e}$ $f_{2 i k}$ indivíduos sejam constrangidos com traço latente $\bar{\theta}_{k}$, para $\mathrm{k}=1, \ldots, \mathrm{q}$, pode ser obtida da distribuição multinomial como

$$
P\left(\boldsymbol{f}_{i} \mid \boldsymbol{\pi}\right)=\frac{n_{i} !}{\prod_{k=1}^{q} f_{1 i k} ! f_{2 i k} !} \prod_{k=1}^{q}\left(\delta_{i} \pi_{k}\right)^{f_{2 i k}}\left[\left(1-\delta_{i}\right) \pi_{k}\right]^{f_{1 i k}}
$$

para $\boldsymbol{f}_{i}=\left(f_{1 i 1}, \ldots, f_{1 i q}, f_{2 i 1}, \ldots, f_{2 i q}\right)$ e $\boldsymbol{\pi}=\left(\pi_{1}, \ldots, \pi_{q}\right)$.

Repare que a distribuição do traço latente é a mesma no grupo de constrangidos e de não constrangidos pelo item. Caso não se queira fazer tal suposição, as probabilidades de classificação dos $n_{i}$ indivíduos nas 2 q possíveis categorias (q para constrangidos + q para não constrangidos) devem ser $\delta_{i} \pi_{k}$ e $\left(1-\delta_{i}\right) \pi_{k}^{*} \operatorname{com~} \mathrm{k}=1, \ldots$, q e $\sum_{k=1}^{q} \pi_{k}=\sum_{k=1}^{q} \pi_{k}^{*}=1$.

Dados $f_{1 i k}, f_{2 i k}$ e $\bar{\theta}_{k}$, a probabilidade de $r_{1 i k}$ indivíduos não cosntrangidos e $r_{2 i k}$ indivíduos constrangidos apresentarem o sintoma i é:

$$
\begin{aligned}
P\left(r_{1 i k}, r_{2 i k} \mid f_{1 i k}, f_{2 i k}, \bar{\theta}_{k}\right)= & \frac{f_{1 i k} ! f_{2 i k} !}{r_{1 i k} !\left(f_{1 i k}-r_{1 i k}\right) ! r_{2 i k} !\left(f_{2 i k}-r_{2 i k}\right) !} \\
& \left(P_{i k}^{*}\right)^{r_{2 i k}}\left(1-P_{i k}^{*}\right)^{f_{2 i k}-r_{2 i k}} P_{i k}^{r_{1 i k}}\left(1-P_{i k}\right)^{f_{1 i k}-r_{1 i k}}
\end{aligned}
$$

$\operatorname{Dados} \overline{\boldsymbol{\theta}}=\left(\bar{\theta}_{1}, \ldots, \bar{\theta}_{q}\right)$ e $\boldsymbol{\pi}$, a probabilidade conjunta de $\boldsymbol{f}=\left(\boldsymbol{f}_{1}, \ldots, \boldsymbol{f}_{I}\right)$ e $\boldsymbol{r}=$ $\left(\boldsymbol{r}_{1}, \ldots, \boldsymbol{r}_{I}\right)$, para $\boldsymbol{r}_{i}=\left(r_{1 i 1}, \ldots, r_{1 i q}, r_{2 i 1}, \ldots, r_{2 i q}\right), \mathrm{i}=1, \ldots, \mathrm{I}$, é dada por:

$$
\begin{aligned}
P(\boldsymbol{f}, \boldsymbol{r} \mid \overline{\boldsymbol{\theta}}, \boldsymbol{\pi}) & =P(\boldsymbol{f} \mid \overline{\boldsymbol{\theta}}, \boldsymbol{\pi}) P(\boldsymbol{r} \mid \boldsymbol{f}, \overline{\boldsymbol{\theta}}, \boldsymbol{\pi}) \\
& =P(\boldsymbol{f} \mid \boldsymbol{\pi}) P(\boldsymbol{r} \mid \boldsymbol{f}, \overline{\boldsymbol{\theta}}) \\
& =\left[\prod_{i=1}^{I} P\left(\boldsymbol{f}_{i} \mid \boldsymbol{\pi}\right)\right]\left[\prod_{i=1}^{I} \prod_{k=1}^{q} P\left(r_{1 i k}, r_{2 i k} \mid f_{1 i k}, f_{2 i k}, \bar{\theta}_{k}\right)\right] .
\end{aligned}
$$


Então, a função log-verossimilhança condicionada aos dados completos é

$$
\begin{aligned}
l(\boldsymbol{\zeta})= & l\left(\boldsymbol{\zeta} \mid \boldsymbol{X}_{. .}, \boldsymbol{C} . ., \boldsymbol{\theta}\right) \\
= & \sum_{i=1}^{I} \ln P\left(\boldsymbol{f}_{i} \mid \boldsymbol{\pi}\right)+\sum_{i=1}^{I} \sum_{k=1}^{q} \ln P\left(r_{1 i k}, r_{2 i k} \mid f_{1 i k}, f_{2 i k}, \bar{\theta}_{k}\right) \\
= & C+\sum_{i=1}^{I} \sum_{k=1}^{q}\left[f_{2 i k} \ln \delta_{i}+f_{1 i k} \ln \left(1-\delta_{i}\right)+r_{2 i k} \ln P_{i k}^{*}+\left(f_{2 i k}-r_{2 i k}\right) \ln \left(1-P_{i k}^{*}\right)+\right. \\
& \left.r_{1 i k} \ln P_{i k}+\left(f_{1 i k}-r_{1 i k}\right) \ln \left(1-P_{i k}\right)\right] \\
= & C+\sum_{i=1}^{I} \sum_{k=1}^{q}\left\{r_{2 i k} \ln \left(\delta_{i} P_{i k}^{*}\right)+\left(f_{2 i k}-r_{2 i k}\right) \ln \left[\delta_{i}\left(1-P_{i k}^{*}\right)\right]+\right. \\
& \left.r_{1 i k} \ln \left[\left(1-\delta_{i}\right) P_{i k}\right]+\left(f_{1 i k}-r_{1 i k}\right) \ln \left[\left(1-\delta_{i}\right)\left(1-P_{i k}\right)\right]\right\}
\end{aligned}
$$

para $\mathrm{C}$ constante em relação ao vetor de parâmetros $\boldsymbol{\zeta}$.

A esperança de 4.38 é

$$
\begin{aligned}
E[l(\boldsymbol{\zeta})]= & \bar{C}+\sum_{i=1}^{I} \sum_{k=1}^{q}\left\{\bar{r}_{2 i k} \ln \left(\delta_{i} P_{i k}^{*}\right)+\left(\bar{f}_{2 i k}-\bar{r}_{2 i k}\right) \ln \left[\delta_{i}\left(1-P_{i k}^{*}\right)\right]+\right. \\
& \left.\bar{r}_{1 i k} \ln \left[\left(1-\delta_{i}\right) P_{i k}\right]+\left(\bar{f}_{1 i k}-\bar{r}_{1 i k}\right) \ln \left[\left(1-\delta_{i}\right)\left(1-P_{i k}\right)\right]\right\}
\end{aligned}
$$

$\operatorname{com} \bar{f}_{1 i k}=E\left[f_{1 i k} \mid \boldsymbol{X}_{. .}, \boldsymbol{C} . ., \boldsymbol{\zeta}\right], \bar{f}_{2 i k}=E\left[f_{2 i k} \mid \boldsymbol{X}_{. .}, \boldsymbol{C} . ., \boldsymbol{\zeta}\right], \bar{r}_{1 i k}=E\left[r_{1 i k} \mid \boldsymbol{X}_{. .}, \boldsymbol{C} . ., \boldsymbol{\zeta}\right]$, $\bar{r}_{2 i k}=E\left[r_{2 i k} \mid \boldsymbol{X}_{. .}, \boldsymbol{C}_{. .}, \boldsymbol{\zeta}\right]$ e $\bar{C}=E\left[C \mid \boldsymbol{X}_{. .}, \boldsymbol{C} . ., \boldsymbol{\zeta}\right]$.

Para maximizar 4.39), primeiramente é necessário obter as derivadas primeira e segunda com relação a $\boldsymbol{\zeta}_{i}$. Especificamente,

$$
\begin{aligned}
& \frac{\partial E[l(\boldsymbol{\zeta})]}{\partial \boldsymbol{\zeta}_{i}}=\sum_{k=1}^{q}\left\{\bar{r}_{2 i k} \frac{1}{\delta_{i} P_{i k}^{*}}\left[\frac{\partial \delta_{i}}{\partial \boldsymbol{\zeta}_{i}} P_{i k}^{*}+\delta_{i} \frac{\partial P_{i k}^{*}}{\partial \boldsymbol{\zeta}_{i}}\right]+\right. \\
& \left(\bar{f}_{2 i k}-\bar{r}_{2 i k}\right) \frac{1}{\delta_{i}\left(1-P_{i k}^{*}\right)}\left[\frac{\partial \delta_{i}}{\partial \boldsymbol{\zeta}_{i}}\left(1-P_{i k}^{*}\right)-\delta_{i} \frac{\partial P_{i k}^{*}}{\partial \boldsymbol{\zeta}_{i}}\right]+ \\
& \left(\bar{r}_{1 i k} \frac{1}{\left(1-\delta_{i}\right) P_{i k}}\left[-\frac{\partial \delta_{i}}{\partial \boldsymbol{\zeta}_{i}} P_{i k}+\left(1-\delta_{i}\right) \frac{\partial P_{i k}}{\partial \boldsymbol{\zeta}_{i}}\right]+\right. \\
& \left.\quad\left(\bar{f}_{1 i k}-\bar{r}_{1 i k}\right) \frac{1}{\left(1-\delta_{i}\right)\left(1-P_{i k}\right)}\left[-\frac{\partial \delta_{i}}{\partial \boldsymbol{\zeta}_{i}}\left(1-P_{i k}\right)+\left(1-\delta_{i}\right) \frac{\partial P_{i k}}{\partial \boldsymbol{\zeta}_{i}}\right]\right\} \\
& =\sum_{k=1}^{q}\left\{\frac{\left(\bar{r}_{2 i k}-\bar{f}_{2 i k} P_{i k}^{*}\right)}{P_{i k}^{*}\left(1-P_{i k}^{*}\right)} \boldsymbol{h}_{i k}^{*}+\frac{\bar{f}_{2 i k}-\left(\bar{f}_{2 i k}+\bar{f}_{1 i k}\right) \delta_{i}}{\delta_{i}\left(1-\delta_{i}\right)} \boldsymbol{h}_{i k(\delta)}^{*}+\frac{\left(\bar{r}_{1 i k}-\bar{f}_{1 i k} P_{i k}\right)}{P_{i k}\left(1-P_{i k}\right)} \boldsymbol{h}_{i k}\right\}
\end{aligned}
$$




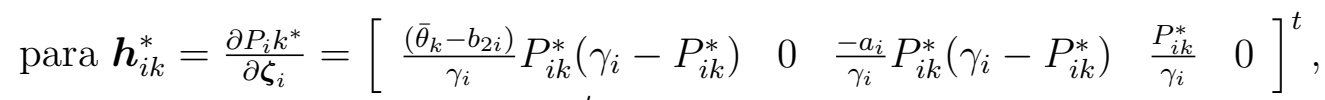

$\boldsymbol{h}_{i k(\delta)}=\frac{\partial \delta_{i}}{\partial \boldsymbol{\zeta}_{i}}=\left[\begin{array}{lllll}0 & 0 & 0 & 0 & 1\end{array}\right]^{t}$,

$\boldsymbol{h}_{i}=\frac{\partial P_{i k}}{\partial \boldsymbol{\zeta}_{i}}=\left[\begin{array}{lllll}\left(\bar{\theta}_{k}-b_{1 i}\right) P_{i k}\left(1-P_{i k}\right) & -a_{i} P_{i k}\left(1-P_{i k}\right) & 0 & 0 & 0\end{array}\right]^{t}$ e para a última igualdade obtida de A.38.

A partir da expressão obtida em A.42, tem-se que

$$
\begin{aligned}
\frac{\partial^{2} E[l(\boldsymbol{\zeta})]}{\partial \boldsymbol{\zeta}_{i} \partial \boldsymbol{\zeta}_{i}^{t}}=\frac{\partial}{\boldsymbol{\zeta}_{i}}\left[\frac{\partial E[l(\boldsymbol{\zeta})]}{\partial \boldsymbol{\zeta}_{i}}\right]^{t} & \\
= & \sum_{k=1}^{q}\left\{\frac{\left(\bar{r}_{2 i k}-\bar{f}_{2 i k} P_{i k}^{*}\right)}{P_{i k}^{*}\left(1-P_{i k}^{*}\right)} \boldsymbol{H}_{i k}^{*}-\frac{\bar{r}_{2 i k}-2 \bar{r}_{2 i k} P_{i k}^{*}+\bar{f}_{2 i k}\left(P_{i k}^{*}\right)^{2}}{\left[P_{i k}^{*}\left(1-P_{i k}^{*}\right)\right]^{2}} \boldsymbol{h}_{i k}^{*}\left(\boldsymbol{h}_{i k}^{*}\right)^{t}+\right. \\
& \quad \frac{\left(\bar{r}_{1 i k}-\bar{f}_{1 i k} P_{i k}\right)}{P_{i k}\left(1-P_{i k}\right)} \boldsymbol{H}_{i k}-\frac{\bar{r}_{1 i k}-2 \bar{r}_{1 i k} P_{i k}+\bar{f}_{1 i k}\left(P_{i k}\right)^{2}}{\left[P_{i k}\left(1-P_{i k}\right)\right]^{2}} \boldsymbol{h}_{i k} \boldsymbol{h}_{i k}^{t}- \\
& \left.\frac{\bar{f}_{2 i k}-2 \bar{f}_{2 i k} \delta_{i}+\left(\bar{f}_{1 i k}+\bar{f}_{2 i k}\right) \delta_{i}^{2}}{\left[\delta_{i}\left(1-\delta_{i}\right)\right]^{2}} \boldsymbol{h}_{i k(\delta)} \boldsymbol{h}_{i k(\delta)}^{t}\right\}
\end{aligned}
$$

com

$$
\begin{aligned}
& \boldsymbol{H}_{i k}^{*}=\frac{\partial^{2} P_{i k}^{*}}{\partial \boldsymbol{\zeta}_{i} \partial \boldsymbol{\zeta}_{i}^{t}}
\end{aligned}
$$

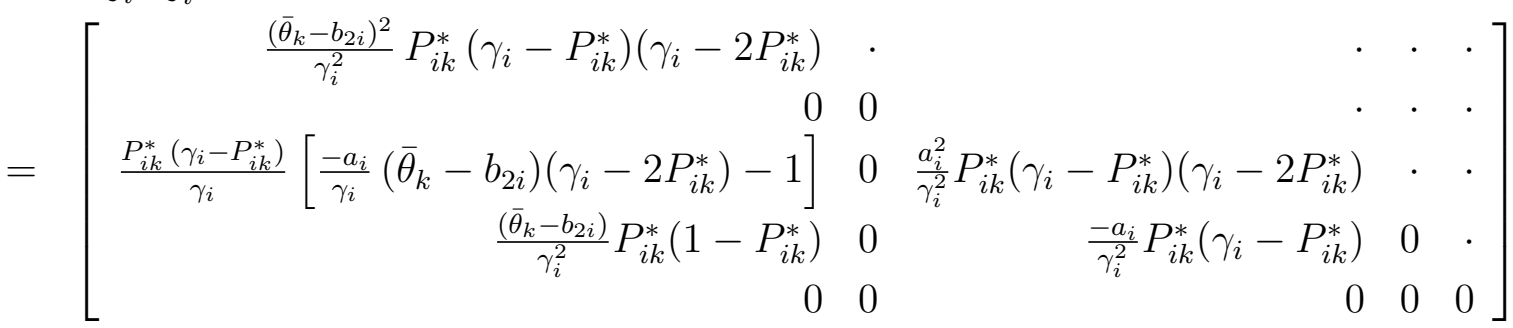

e

$$
\begin{aligned}
& \boldsymbol{H}_{i k}=\frac{\partial^{2} P_{i k}}{\partial \boldsymbol{\zeta}_{i} \partial \boldsymbol{\zeta}_{i}^{t}} \\
& =\left[\begin{array}{rrrcc}
\left(\bar{\theta}_{k}-b_{1 i}\right)^{2} P_{i k}\left(1-P_{i k}\right)\left(1-2 P_{i k}\right) & \cdot & \cdot & \cdot & \cdot \\
-P_{i k}\left(1-P_{i k}\right)\left[1+a_{i}\left(\bar{\theta}_{k}-b_{1 i}\right)\left(1-2 P_{i k}\right)\right] & a_{i}^{2} P_{i k}\left(1-P_{i k}\right)\left(1-2 P_{i k}\right) & \cdot & \cdot & . \\
0 & 0 & 0 & \cdot & \cdot \\
0 & 0 & 0 & 0 & \cdot \\
0 & 0 & 0 & 0 & 0
\end{array}\right]
\end{aligned}
$$

O algoritmo EM consiste na aplicação iterativa dos seguintes passos:

Passo E: Fixar os pontos de quadratura $\bar{\theta}_{k}$, os pesos $A_{k}^{(t)}$, para $\mathrm{k}=1, \ldots$ q, e as estimativas dos parâmetros dos itens, $\hat{\boldsymbol{\zeta}}_{i}^{(t)}$, no passo $(\mathrm{t})$, para $\mathrm{i}=1, \ldots$, I, e gerar $g_{j}^{*}\left(\bar{\theta}_{k}\right)^{(t)}$, $\bar{f}_{1 i k}^{(t)}, \bar{f}_{2 i k}^{(t)}, \bar{r}_{1 i k}^{(t)}$ e $\bar{r}_{2 i k}^{(t)}$. 
Passo M: Com os valores gerados no Passo E, resolver as equações de estimação para $\boldsymbol{\zeta}_{i}, \mathrm{i}=1, \ldots$ I, usando o algoritmo de Newton-Raphson ou Scoring de Fisher.

O procedimento é repetido até a convergência ser atingida segundo algum critério previamente estabelecido.

\subsection{Itens e Habilidades Desconhecidos - Monte Carlo via Cadeia de Markov (MCMC)}

O objetivo deste método é estimar os valores de $\theta_{j}$ e $\boldsymbol{\zeta}_{\boldsymbol{i}}$, cujas distribuições a posteriori condicionadas aos dados observados são definidas como as distribuições estacionárias de uma cadeia de Markov. Uma descrição do método pode ser encontrada em Ross (1997) e, particularmente no contexto da TRI, em Patz \& Junker (1999).

Pela independência entre as respostas de diferentes indivíduos, a função de verossimilhança é dada por:

$$
L(\boldsymbol{\theta}, \boldsymbol{\zeta})=\prod_{i=1}^{I} \prod_{j=1}^{n}\left[\left(P_{i j}^{*}\right)^{x_{i j}}\left(1-P_{i j}^{*}\right)^{\left(1-x_{i j}\right)} \delta_{i}\right]^{c_{i j}} \times\left[P_{i j}^{x_{i j}}\left(1-P_{i j}\right)^{\left(1-x_{i j}\right)}\left(1-\delta_{i}\right)\right]^{1-c_{i j}} .
$$

Denotando-se a distribuição a priori para $\boldsymbol{\theta}$ e $\boldsymbol{\zeta}$ por $\pi(\boldsymbol{\theta}, \boldsymbol{\zeta})$ e assumindo-se que $\pi(\boldsymbol{\theta}, \boldsymbol{\zeta})=$ $\prod_{j=1}^{n} \pi\left(\theta_{j}\right) \prod_{i=1}^{I} \pi\left(\boldsymbol{\zeta}_{i}\right)$, a função de distribuição a posteriori é igual a:

$$
f(\boldsymbol{\theta}, \boldsymbol{\zeta}) \propto L(\boldsymbol{\theta}, \boldsymbol{\zeta}) \prod_{j=1}^{n} \pi\left(\theta_{j}\right) \prod_{i=1}^{I} \pi\left(\boldsymbol{\zeta}_{i}\right)
$$

$\operatorname{Para} q_{\theta}\left(\theta_{j}^{(k-1)}, \theta_{j}^{(k)}\right)$ e $q_{\boldsymbol{\zeta}}\left(\boldsymbol{\zeta}_{i}^{(k-1)}, \boldsymbol{\zeta}_{i}^{(k)}\right)$ sendo as densidades propostas relativas aos parâmetros individuais e de item (com $\mathrm{k}$ denotando a iteração), respectivamente, o algoritmo pode ser descrito como:

1. Simulação dos parâmetros individuais $\theta_{j}$, para $\mathrm{j}=1, \ldots, \mathrm{n}$ :

- Simula-se $\theta_{j}^{*}$ de uma distribuição $q_{\theta}\left(\theta_{j}^{(k-1)}, \theta_{j}^{*}\right)$

- Aceita-se $\theta_{j}^{(k)}=\theta_{j}^{*}$ com probabilidade

$$
\alpha_{j}\left(\theta_{j}^{(k-1)}, \theta_{j}^{*}\right)=\min \left\{\frac{L\left(\boldsymbol{\theta}_{j}^{*}, \boldsymbol{\zeta}^{(k-1)}\right) \pi\left(\theta_{j}^{*}\right) q_{\theta}\left(\theta_{j}^{*}, \theta_{j}^{(k-1)}\right)}{L\left(\boldsymbol{\theta}_{j}^{(k-1)}, \boldsymbol{\zeta}^{(k-1)}\right) \pi\left(\theta_{j}^{(k-1)}\right) q_{\theta}\left(\theta_{j}^{(k-1)}, \theta_{j}^{*}\right)}, 1\right\}
$$


- Segue-se para o passo 2

2. Simulação dos parâmetros de item $\boldsymbol{\zeta}_{i}$, para $\mathrm{i}=1, \ldots, \mathrm{I}$ :

- Simula-se $\boldsymbol{\zeta}_{i}^{*}$ de uma distribuição $q_{\boldsymbol{\zeta}}\left(\boldsymbol{\zeta}_{i}^{(k-1)}, \boldsymbol{\zeta}_{i}^{*}\right)$

- Aceita-se $\boldsymbol{\zeta}_{i}^{(k)}=\boldsymbol{\zeta}_{i}^{*}$ com probabilidade

$$
\alpha_{i}\left(\boldsymbol{\zeta}_{i}^{(k-1)}, \boldsymbol{\zeta}_{i}^{*}\right)=\min \left\{\frac{L\left(\boldsymbol{\theta}^{(k)}, \boldsymbol{\zeta}_{i}^{*}\right) \pi\left(\boldsymbol{\zeta}_{i}^{*}\right) q_{\boldsymbol{\zeta}}\left(\boldsymbol{\zeta}_{i}^{*}, \boldsymbol{\zeta}_{i}^{(k-1)}\right)}{L\left(\boldsymbol{\theta}^{(k)}, \boldsymbol{\zeta}_{i}^{(k-1)}\right) \pi\left(\boldsymbol{\zeta}_{i}^{(k-1)}\right) q_{\boldsymbol{\zeta}}\left(\boldsymbol{\zeta}_{i}^{(k-1)}, \boldsymbol{\zeta}_{i}^{*}\right)}, 1\right\}
$$

- Segue-se para o passo 1

Duas vantagens podem ser apontadas para o método de estimação MCMC. A primeira é a obtenção de uma aproximação das distribuições a posteriori dos parâmetros de interesse e não apenas estimativas pontuais, como nos métodos anteriores. A segunda é que as estimativas podem ser obtidas para itens ou indivíduos em que todas as respostas são iguais (a 0 ou 1$)$. 


\section{Capítulo 5}

\section{Exemplos com Amostras Simuladas}

Neste capítulo, serão realizadas aplicações do modelo (3.3) a dados simulados com o propósito de verificar a qualidade das estimativas dos respectivos parâmetros dos itens e individuais. Serão gerados 10 conjuntos de dados com diferentes números de indivíduos e de itens com funcionamento diferencial. Com base nessas respostas simuladas, o método MCMC será adotado para a estimação dos parâmetros.

Para tanto, serão considerados instrumentos com 20 itens, respondidos por $n=300,500$ e 1000 indivíduos, variando-se o número $(\mathrm{k})$ de itens com funcionamento diferencial de 1 a 20. A escolha do número total de itens foi baseada nas escalas psiquiátricas descritas em Gorenstein et al. (1999). Das 49 escalas com itens de respostas dicotômicas ou dicotomizáveis, $8(16 \%)$ apresentam menos de 15 itens, 19 (39\%) são compostas de 15 a 24 itens, $12(24 \%)$ apresentam de 25 a 34 itens, $6(12 \%)$ contêm de 35 a 44 itens e as 4 restantes (8\%) são formadas por 45 itens ou mais. O intervalo de 300 a 1000 indivíduos respondedores é consonante com os tamanhos amostrais relatados nas pesquisas da área. A Tabela 5.1 indica as 10 combinações (cenários) de tamanho de amostra e número de itens com funcionamento diferencial empregadas na simulação. Repare que o procedimento adotado difere das simulações no sentido usual, pois apenas um conjunto de dados será gerado para cada cenário. Para gerar as respostas de cada cenário, os valores dos parâmetros de item foram fixados conforme indicado na Tabela 5.2. Nas simulações em que $k<20$, as variáveis respostas foram geradas considerando os $\mathrm{k}$ primeiros itens como itens com funcionamento diferencial e os demais sem funcionamento diferencial. Portanto, os parâmetros $\delta_{i}, \gamma_{i}$ e $b_{2 i}$ não são definidos, para $i=k+1, \ldots, 20$. Os programas para as simulações de $C_{i j}$ e $X_{i j}$, definidos em (3.1), foram elaborados no software SAS versão 8 (SAS Institute Inc. 1999) e seguiram os seguintes passos:

- Fixaram-se n, k e os valores dos parâmetros de item (Tabela 5.2);

- Geraram-se n valores de uma variável com distribuição Normal(0,1). Tais valores 
Tabela 5.1: Cenário utilizado para a geração das amostras simuladas para um questionário com 20 itens.

\begin{tabular}{cccc}
\hline & $\mathrm{n}=300$ & $\mathrm{n}=500$ & $\mathrm{n}=1000$ \\
\hline $\mathrm{k}=1$ & $\mathrm{X}$ & $\mathrm{X}$ & \\
$\mathrm{k}=2$ & $\mathrm{X}$ & $\mathrm{X}$ & \\
$\mathrm{k}=6$ & $\mathrm{X}$ & $\mathrm{X}$ & $\mathrm{X}$ \\
$\mathrm{k}=10$ & & $\mathrm{X}$ & $\mathrm{X}$ \\
$\mathrm{k}=20$ & & & $\mathrm{X}$ \\
\hline
\end{tabular}

n: número de indivíduos;

k: número de itens com funcionamento diferencial.

correspondem aos traços latentes $\theta_{j}$, para $\mathrm{j}=1, \ldots, \mathrm{n}$;

- Para cada $\delta_{i}$ fixado, $\mathrm{i}=1, \ldots, \mathrm{k}$, geraram-se $\mathrm{n}$ observações de uma variável com distribuição de $\operatorname{Bernoulli}\left(\delta_{i}\right)$. Tais observações correspondem às respostas $C_{i j}$, para $\mathrm{i}=1$, $\ldots, \mathrm{k} \mathrm{e} \mathrm{j}=1, \ldots, \mathrm{n}$. Quando $k<20, C_{i j}=0$, para $\mathrm{i}=\mathrm{k}+1, \ldots, 20$ e $\mathrm{j}=1, \ldots, \mathrm{n}$;

- Com os valores selecionados para $\boldsymbol{\zeta}_{i}=\left(a_{i}, b_{1 i}, b_{2 i}, \gamma_{i}, \delta_{i}\right), \mathrm{i}=1, \ldots, \mathrm{k}$, e gerados para $\theta_{j}, \mathrm{j}=1, \ldots, \mathrm{n}$, calcularam-se $P_{i j}$ e $P_{i j}^{*}$, de acordo com as equações 3.1) e (3.2);

- Quando $k<20$, com os valores selecionados para $\boldsymbol{\zeta}_{i}=\left(a_{i}, b_{1 i}\right)$, $\mathrm{i}=\mathrm{k}+1, \ldots, 20$, e gerados para $\theta_{j}, \mathrm{j}=1, \ldots, \mathrm{n}$, calculou-se $P_{i j}$, de acordo com a equação 3.1);

- Para $\mathrm{i}=1, \ldots, 20$ e $\mathrm{j}=1, \ldots, \mathrm{n}$, se $C_{i j}=1$, gerou-se a resposta $X_{i j}$ com distribuição $\operatorname{Bernoulli}\left(P_{i j}^{*}\right)$, e, se $C_{i j}=0$, gerou-se $X_{i j} \operatorname{com}$ distribuição $\operatorname{Bernoulli}\left(P_{i j}\right)$.

Particularmente para a simulação das respostas de 500 indivíduos ao questionário de 20 itens com os primeiros 10 deles com funcionamento diferencial, a equação (3.2), que define $P_{i j}^{*}$, foi considerada substituindo-se o parâmetro $\gamma_{i}$ pela constante 1 , para $\mathrm{i}=1, \ldots, 20$. Isto significa que o parâmetro $\gamma_{i}$ foi eliminado do modelo nesta simulação. $\mathrm{O}$ intuito da exclusão desses parâmetros foi averiguar se uma simplifcação do modelo proposto melhora a qualidade das estimativas.

O programa WinBUGS versão 1.4.1 (Spiegelhalter, Thomas, Best \& Lunn 2003) foi utilizado para a obtenção das estimativas dos parâmetros pelo método MCMC. As distribuições a priori consideradas para os parâmetros $a_{i}, b_{1 i}$ e $b_{2 i^{\prime}}, \gamma_{i^{\prime}}, \delta_{i^{\prime}}$ e $\theta_{j}, \mathrm{i}=1, \ldots$, $20, i^{\prime}=1, \ldots, \mathrm{k} \mathrm{e} \mathrm{j}=, 1, \ldots, \mathrm{n}$, foram $\log \operatorname{Normal}(0,4), \operatorname{Normal}(0,4), \operatorname{Beta}(4,1), \operatorname{Beta}(2,2){ }^{1}$

\footnotetext{
${ }^{1}$ As distribuições foram definidas de tal forma que os valores esperados são dados por $\mathrm{E}\left[\gamma_{i^{\prime}}\right]=0.8 \mathrm{e}$ $\mathrm{E}\left[\delta_{i^{\prime}}\right]=0.5$
} 
Tabela 5.2: Valores fixados para os parâmetros de item para a simulação das respostas. (a: parâmetro de discriminação; $b_{1}$ : parâmetro de gravidade de depressão para indivíduos não constrangidos; $b_{2}$ : parâmetro de gravidade de depressão para indivíduos constrangidos; $\gamma$ : probabilidade de um indivíduo constrangido admitir que tem o sintoma; $\delta$ : probabilidade de o item constranger o indivíduo)

\begin{tabular}{cccccc}
\hline Item & $\mathrm{a}$ & $b_{1}$ & $b_{2}$ & $\gamma$ & $\delta$ \\
\hline 1 & 1.42 & 0.84 & 3.52 & 0.80 & 0.50 \\
2 & 1.25 & 0.72 & 1.52 & 0.85 & 0.60 \\
3 & 0.55 & 1.64 & 2.40 & 0.95 & 0.50 \\
4 & 0.81 & -1.55 & -0.50 & 0.90 & 0.40 \\
5 & 1.05 & 0.47 & -0.40 & 0.70 & 0.70 \\
6 & 2.04 & -0.11 & 0.80 & 0.75 & 0.25 \\
7 & 1.04 & 0.55 & -0.40 & 0.78 & 0.76 \\
8 & 2.25 & 0.11 & 1.10 & 0.81 & 0.36 \\
9 & 0.76 & -1.16 & 0.50 & 0.92 & 0.45 \\
10 & 2.29 & -0.20 & 1.80 & 0.77 & 0.39 \\
11 & 1.20 & -0.08 & -0.08 & 0.81 & 0.45 \\
12 & 0.95 & -1.07 & -1.07 & 0.83 & 0.58 \\
13 & 1.47 & 1.61 & 1.61 & 0.91 & 0.52 \\
14 & 0.93 & -0.02 & -0.02 & 0.75 & 0.25 \\
15 & 1.57 & -0.96 & -0.96 & 0.73 & 0.34 \\
16 & 0.80 & 1.57 & 1.57 & 0.87 & 0.63 \\
17 & 1.05 & -0.92 & -0.92 & 0.97 & 0.72 \\
18 & 2.42 & -0.44 & -0.44 & 0.99 & 0.40 \\
19 & 1.80 & 1.03 & 1.03 & 0.65 & 0.36 \\
20 & 1.18 & 0.64 & 0.64 & 0.76 & 0.27 \\
\hline mínimo & 0.55 & -1.55 & -1.07 & 0.65 & 0.25 \\
maximo & 2.42 & 1.64 & 3.52 & 0.99 & 0.76 \\
\hline & & & & &
\end{tabular}


Tabela 5.3: Estimativas dos parâmetros $b_{2 i}, \gamma_{i}$ e $\delta_{i}$, para $i=1,2$, nas simulações com 1 e 2 itens com funcionamento diferencial $(k)$.

\begin{tabular}{|c|c|c|c|c|c|}
\hline \multirow[b]{3}{*}{ Parâmetro } & \multirow[b]{3}{*}{ Valores Reais } & \multicolumn{4}{|c|}{ Valores estimados } \\
\hline & & \multicolumn{2}{|c|}{$n=300$} & \multicolumn{2}{|c|}{$\mathrm{n}=500$} \\
\hline & & $\mathrm{k}=1$ & $\mathrm{k}=2$ & $\mathrm{k}=1$ & $\mathrm{k}=2$ \\
\hline$b_{21}$ & 3,52 & 3,33 & 3,27 & 2,91 & 4,01 \\
\hline$b_{22}$ & 1,52 & - & 1,41 & - & 1,48 \\
\hline$\gamma_{1}$ & 0,80 & 0,77 & 0,77 & 0,78 & 0,76 \\
\hline$\gamma_{2}$ & 0,85 & - & 0,82 & - & 0,79 \\
\hline$\delta_{1}$ & 0,50 & 0,53 & 0,50 & 0,51 & 0,52 \\
\hline$\delta_{2}$ & 0,60 & - & 0,59 & - & 0,60 \\
\hline
\end{tabular}

e $\operatorname{Normal}(0,1)$, respectivamente. Essas distribuições foram escolhidas segundo os valores que se esperam para tais parâmetros (vide seção 2.2) e são as mesmas estabelecidas no programa Bilog na estimação pelo método bayesiano marginal. Em alguns casos, pequenas modificações na variância do parâmetro $a_{i}$ foram introduzidas por razões operacionais (nas situações em que o algoritmo não convergiu).

Foram geradas duas cadeias de Markov e a convergência foi verificada através do traço da estatística de Gelman \& Rubin (1992) para os parâmetros de item. O descarte (Burn-in) foi fixado em 4000 ou 4500 iterações, dependendo da convergência (estatística de Gelman \& Rubin (1992) próxima a 1 e estabilização dos valores das estimativas ao longo das iterações). Geraram-se 8000 iterações adicionais após o descarte e um salto de 10 ou 20 foi determinado na tentativa de reduzir a dependência das cadeias. O tamanho amostral final para o cálculo das estimativas de cada parâmetro resultou, portanto, em 800 valores $(2 \times 8000 / 20)$.

As Figuras 5.1 a 5.8 mostram os valores reais dos parâmetros e calculados por MCMC para cada simulação realizada. Para as simulações com 1 ou 2 itens diferenciais, as estimativas de $b_{2}, \gamma$ e $\delta$ encontram-se apresentadas na Tabela 5.3. Adicionalmente, os desvios (módulo da diferença entre os valores estimado e real do parâmetro) das estimativas de todos os parâmetros $a_{i}, b_{1 i}, b_{2 i^{\prime}}, \gamma_{i^{\prime}}, \delta_{i^{\prime}}$ e $\theta_{j}$, para $\mathrm{i}=1, \ldots, 20, \mathrm{i}=1, \ldots, \mathrm{k} \mathrm{e} \mathrm{j}=1, \ldots, \mathrm{n}$, também foram calculados. A Tabela 5.4 apresenta os valores médios, mínimos, medianos, máximos e percentis 75 dos desvios para cada tipo de parâmetro do modelo. A Figura 5.9 representa os desvios médios. Pode-se perceber que:

- As estimativas de $a, b_{1}, b_{2}$ e $\delta$ são próximas dos valores reais em todas as simulações, 
com correlações de 0,86 a 0,98, 0,98 a 0.99, 0,95 a 0,99, e 0,97 a 0,99, respectivamente, e desvios de baixa magnitude.

- Apenas para o parâmetro $\theta$, os desvios máximos apresentam-se altos, apesar das elevadas correlações com os valores reais (de 0,90 a 0,93 ). Por outro lado, para a maioria da amostra, os desvios têm baixa magnitude (mediana variando de 0,234 a $0,281$ e percentil 75 variando de 0,426 a 0,474$)$.

- Com a diminuição do número de indivíduos e com o aumento do número de itens com funcionamento diferencial, nota-se um crescimento sistemático dos desvios das estimativas de $a$ e de $b_{1}$ (Figura 5.9). No primeiro caso, observa-se, ainda, o decrescimento da correlação com seus valores verdadeiros;

- As piores estimativas foram as dos parâmetros $\gamma$; as correlações com os valores reais variaram de 0,30 a 0,72. Quanto maior o número de indivíduos e menor o número de itens com funcionamento diferencial, melhores as estimativas. Em especial, nas simulações com 1 ou 2 itens com funcionamento diferencial, as estimativas de $\gamma$ foram próximas aos valores reais (Tabela 5.3);

- As estimativas obtidas na simulação em que se eliminaram os parâmetros $\gamma_{i}, \mathrm{i}=1$, ..., 20, do modelo (ou seja, em que se supôs que a probabilidade de um indivíduo constrangido responder que apresenta o sintoma dado que realmente o apresenta é igual a 1) foram bastante boas. Apresentaram altas correlações com os valores reais (de 0,92 a 0,99 ) e baixos desvios, mesmo com $50 \%$ dos itens considerados com funcionamento diferencial.

De uma forma geral, pode-se notar que o maior problema está na estimação dos parâmetros $\gamma$. Para o processo de estimação adotado, modelos com poucos itens de funcionamento diferencial suscetíveis à mentira (apenas 1 ou 2) apresentaram ajustes adequados. Um estudo mais amplo, incluindo-se outros métodos de estimação e simulações para as comparações dos vícios e dos erros quadráticos médios dos diferentes estimadores, pode ser sugerido para a melhora do processo de estimação em casos mais gerais. 

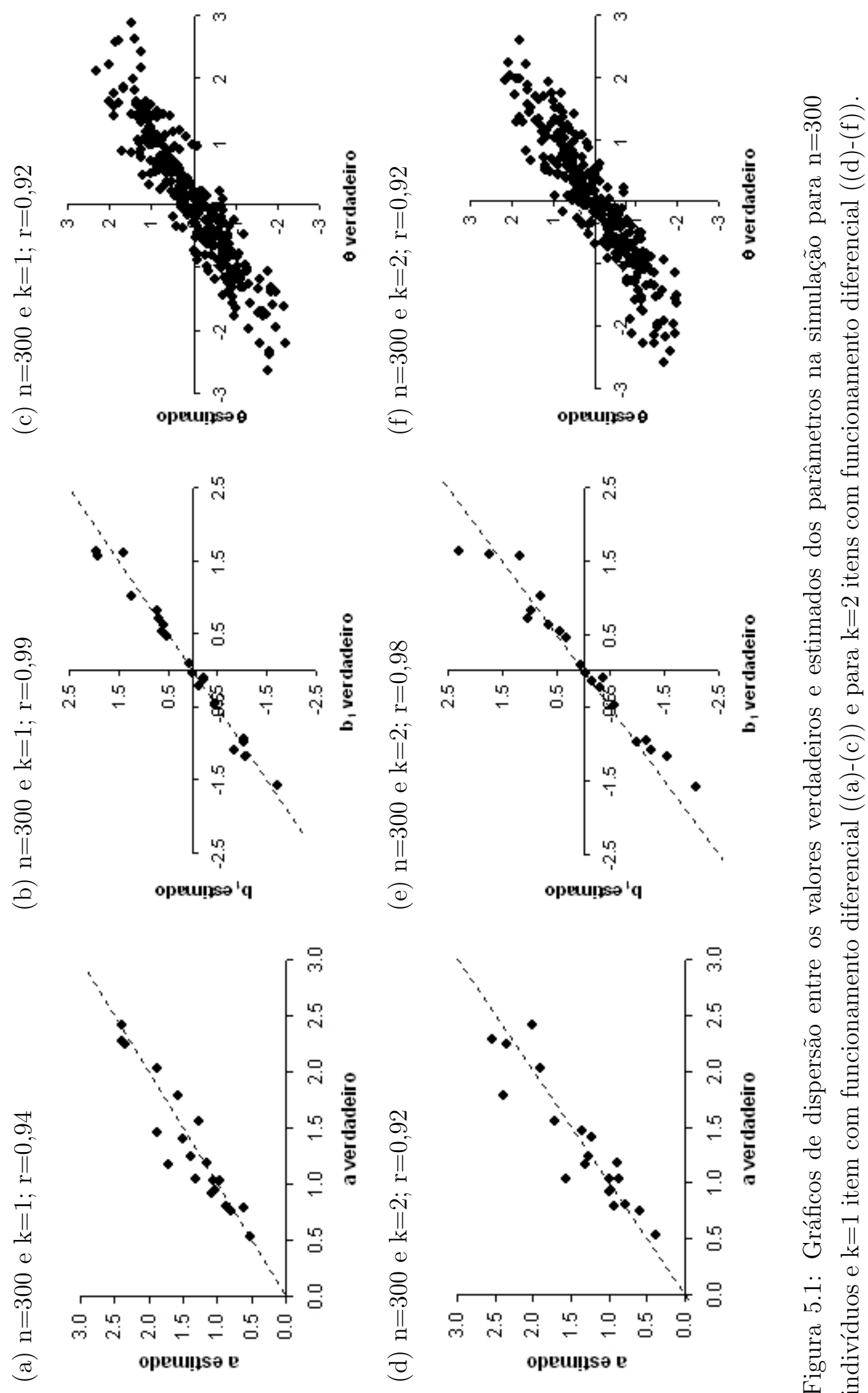

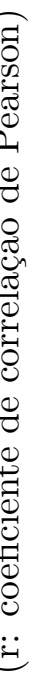



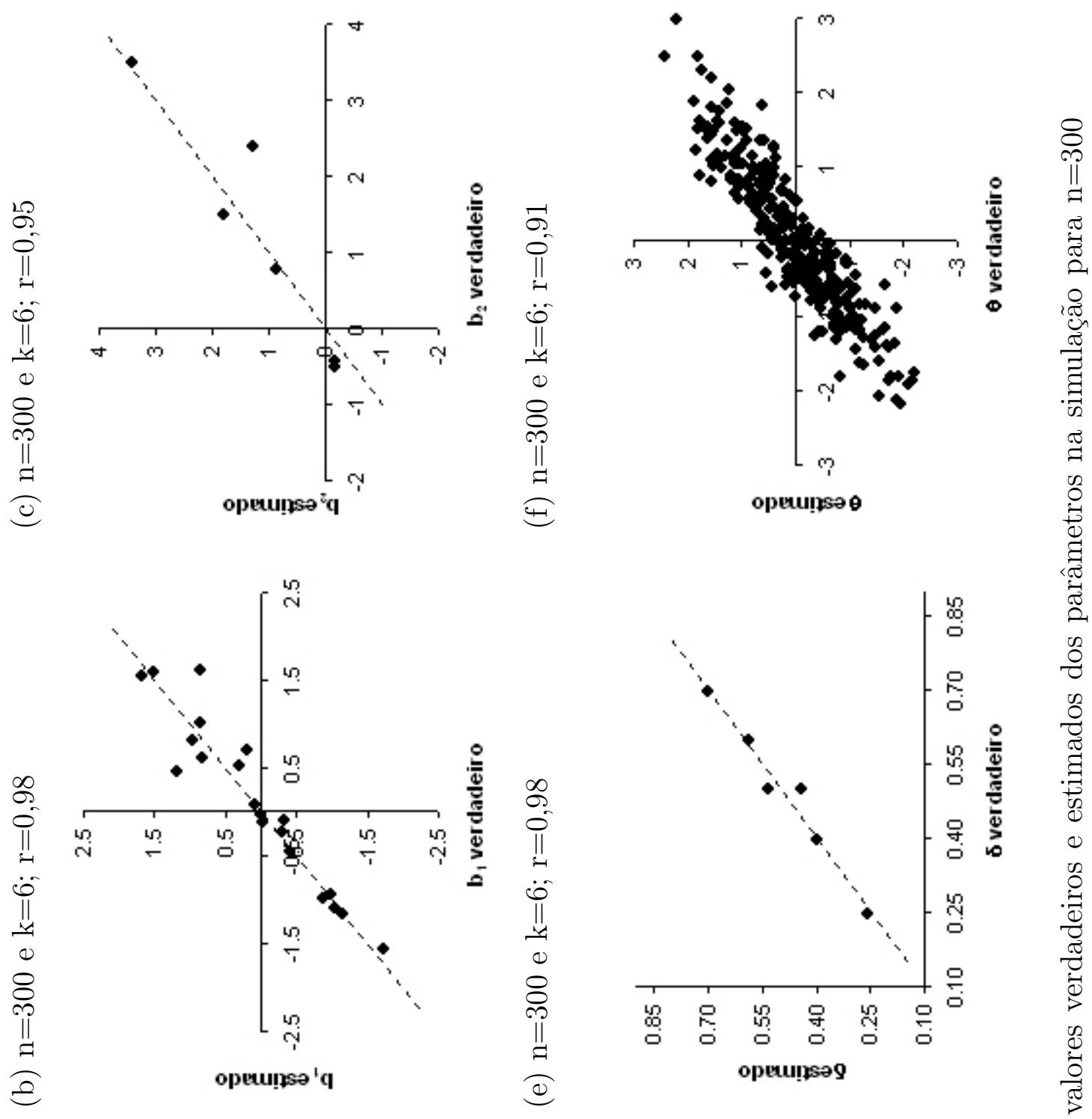

莒

$\Theta$

过
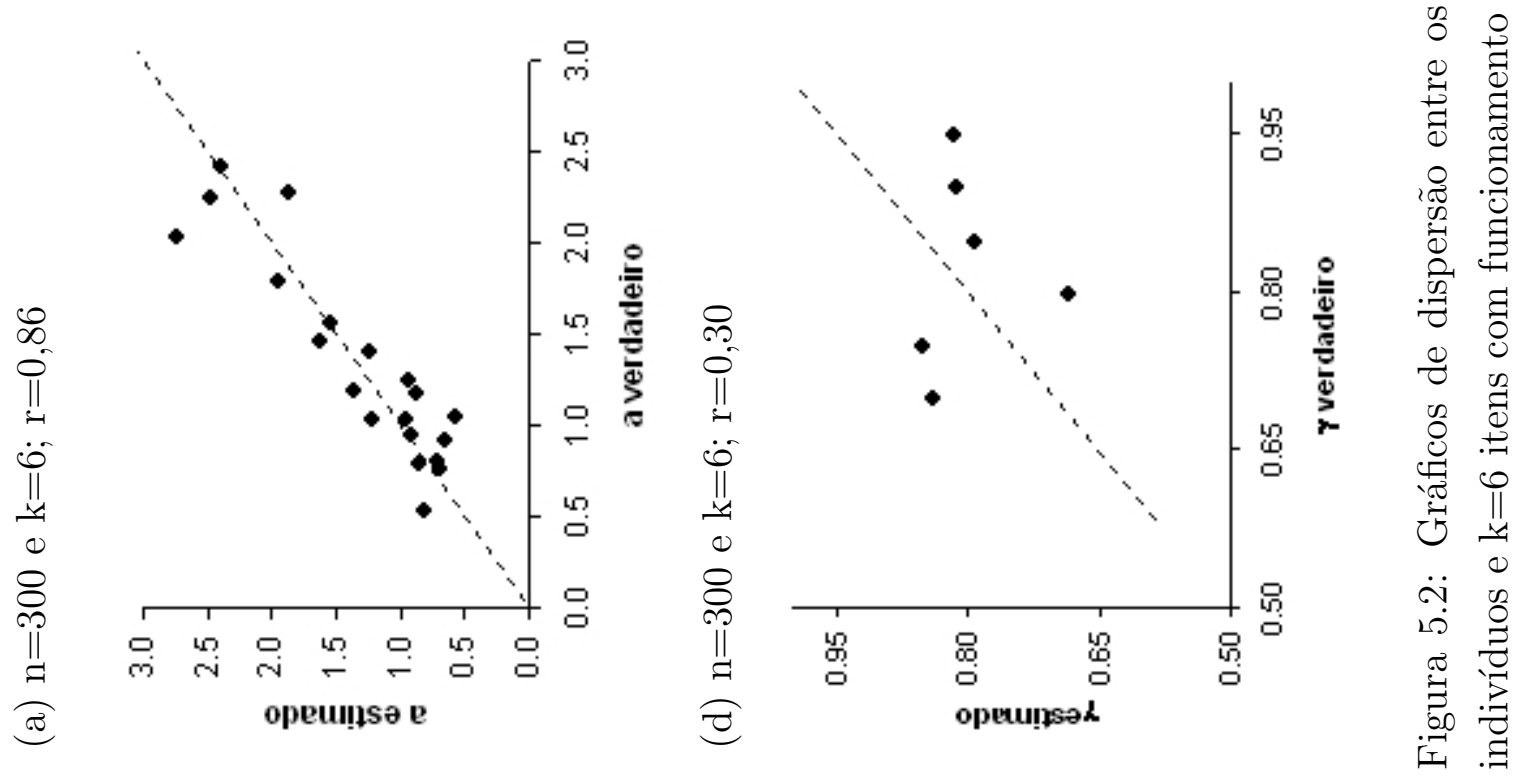

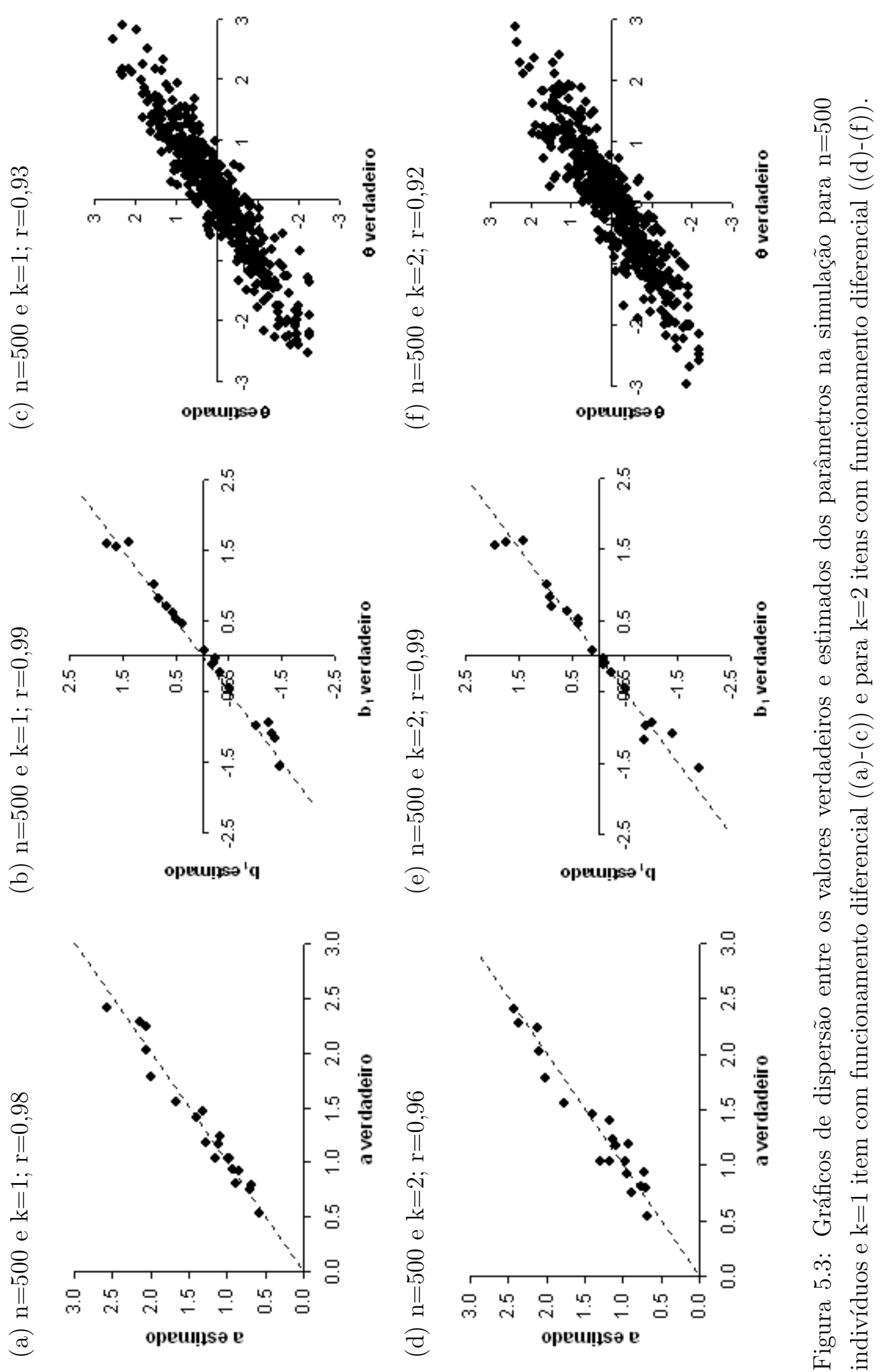

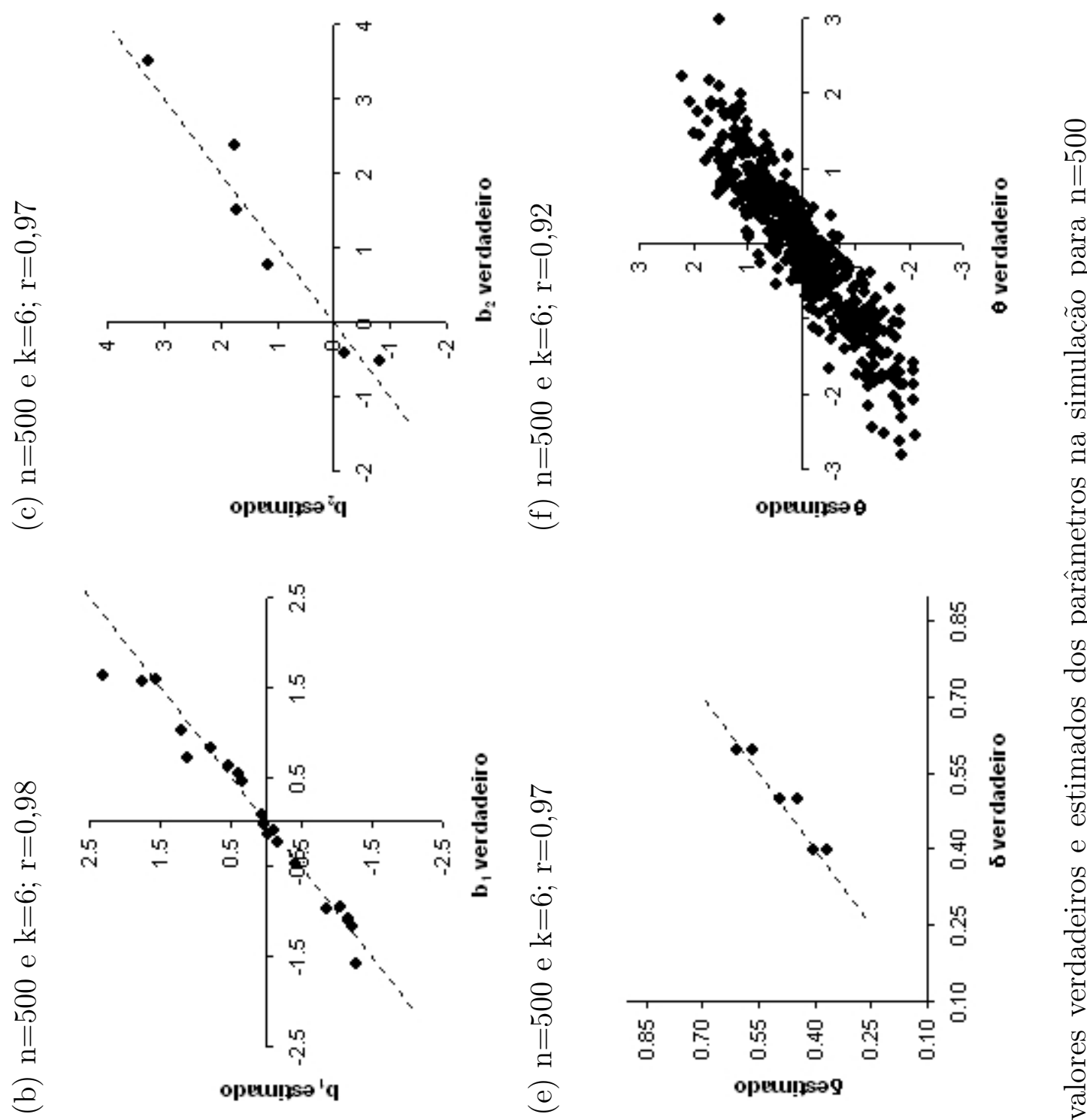

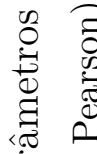

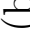

opeunse '
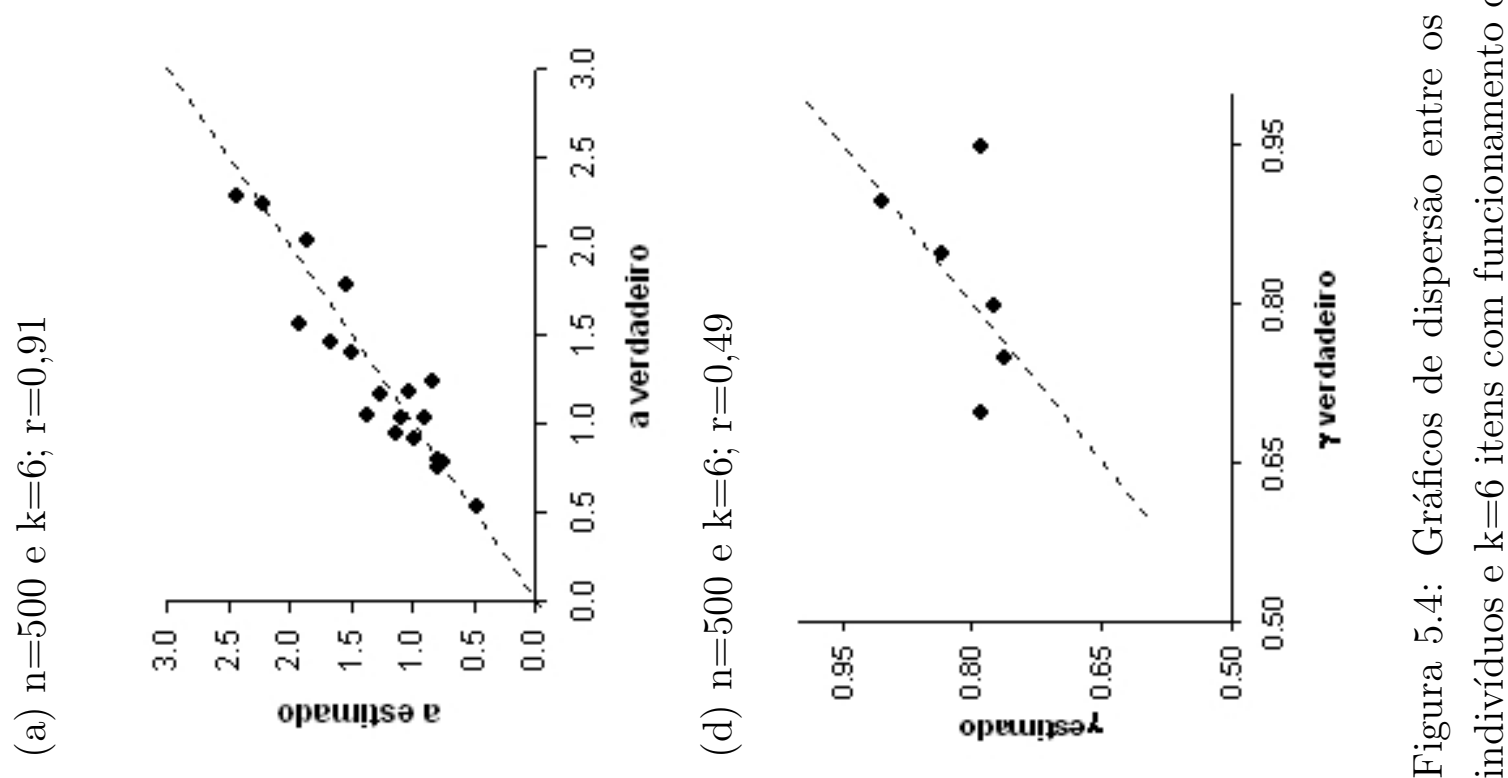

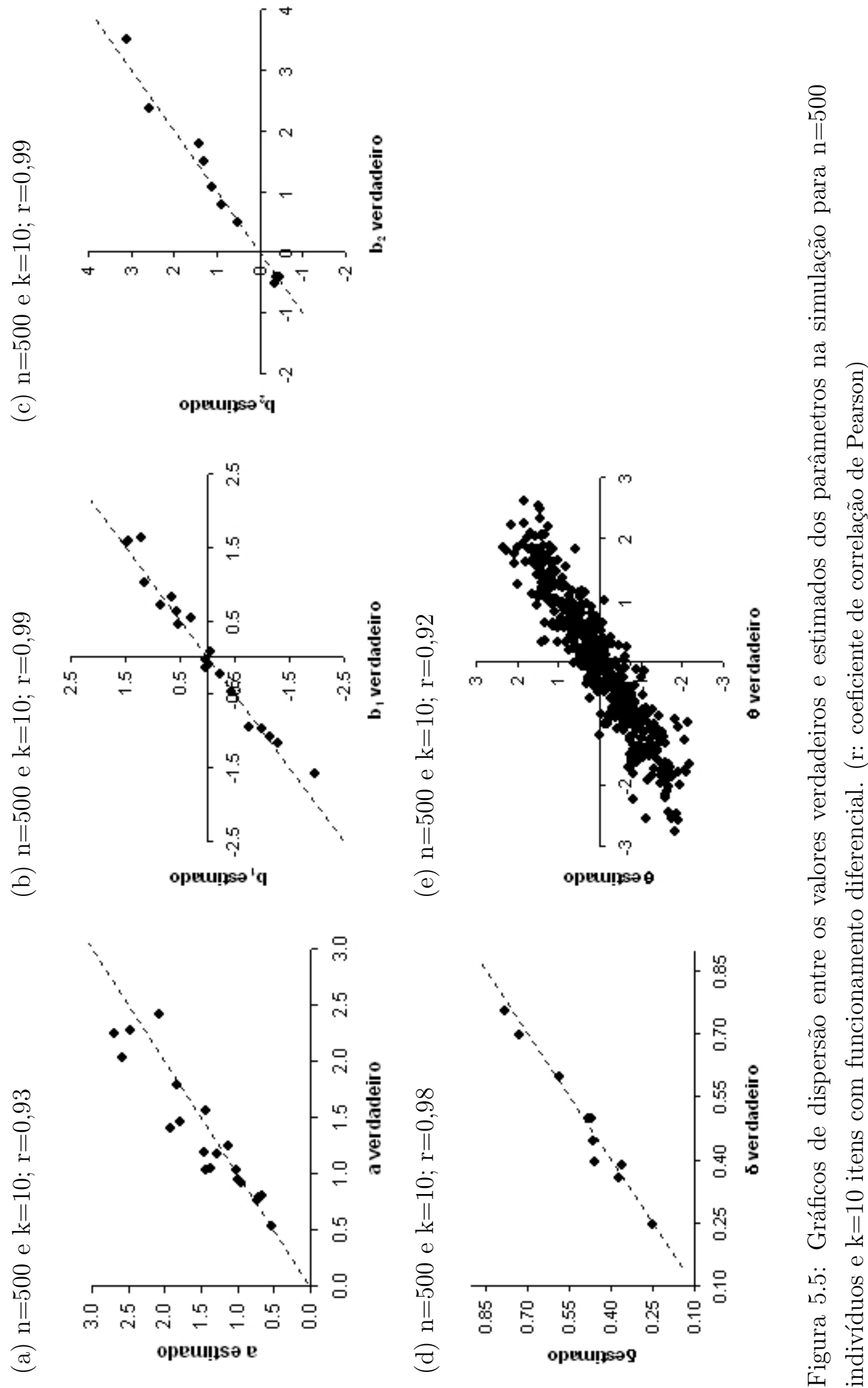

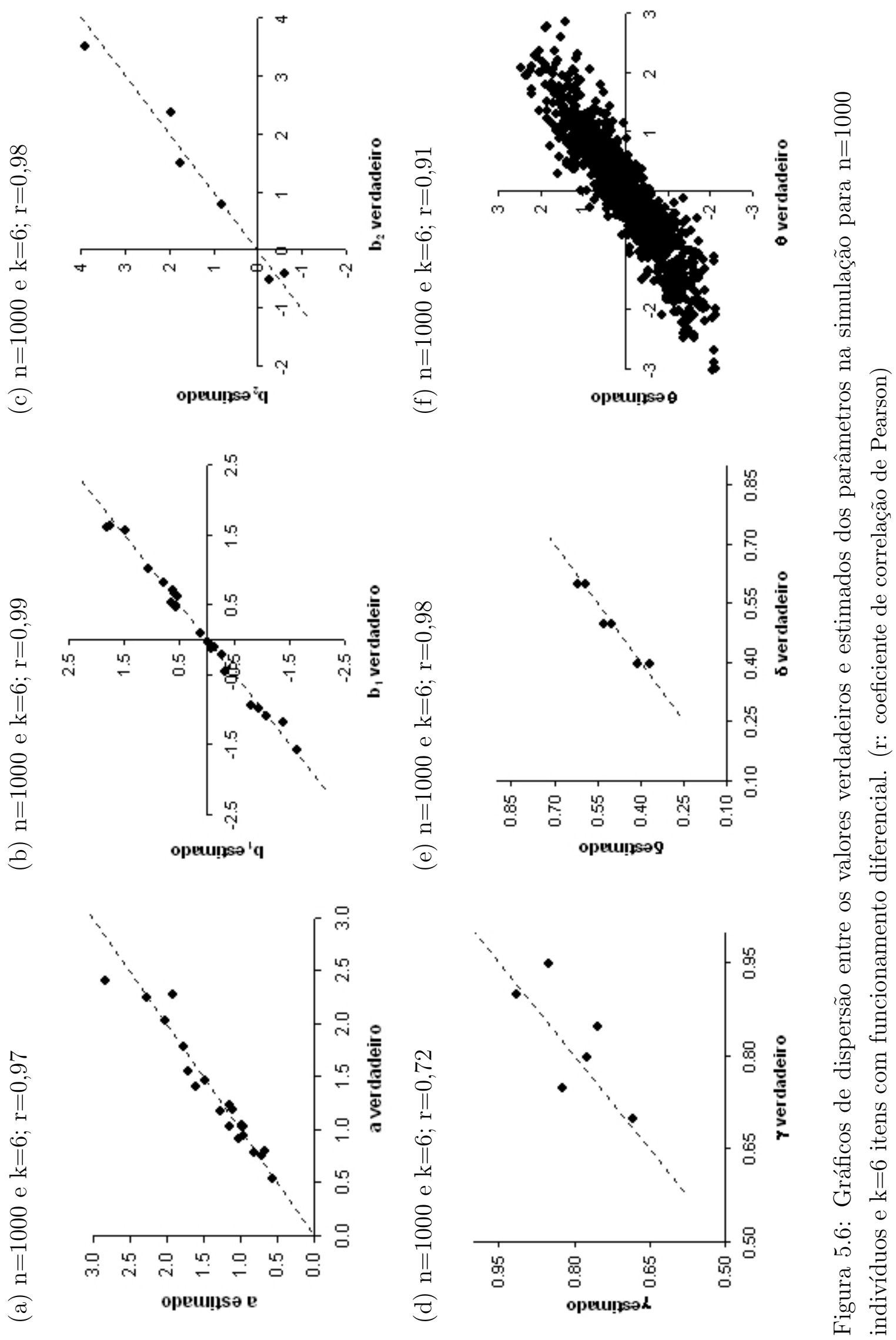

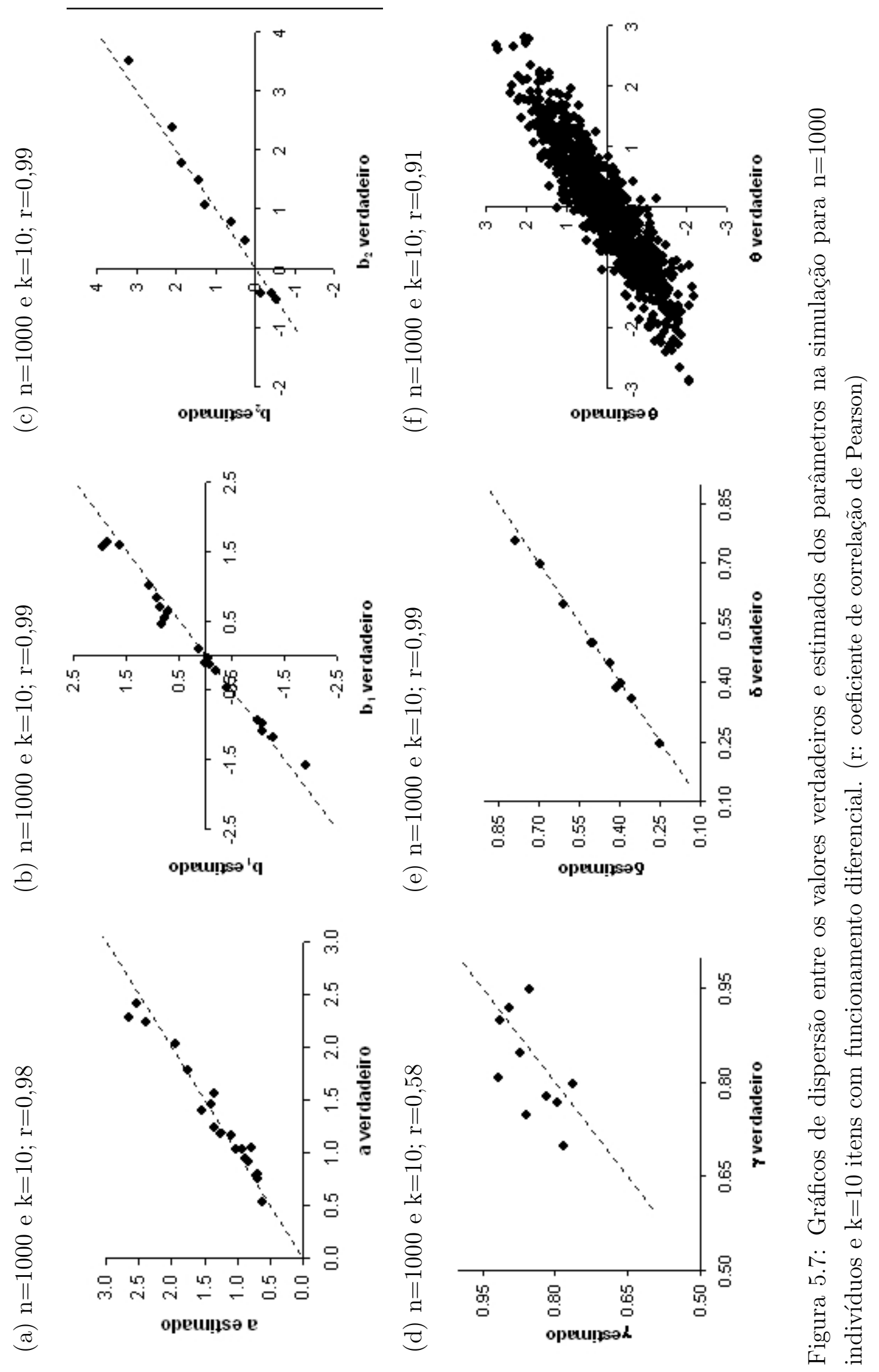

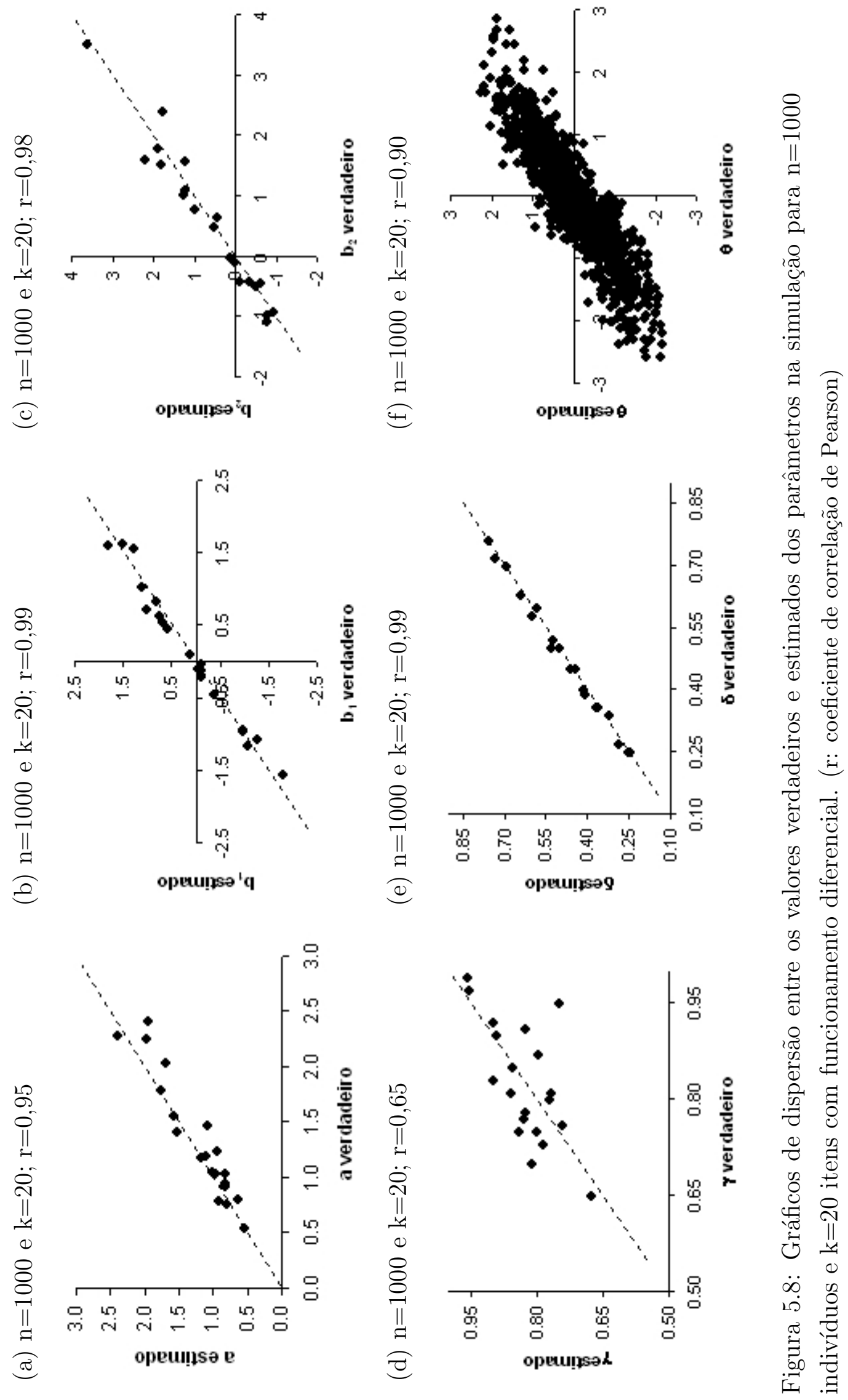


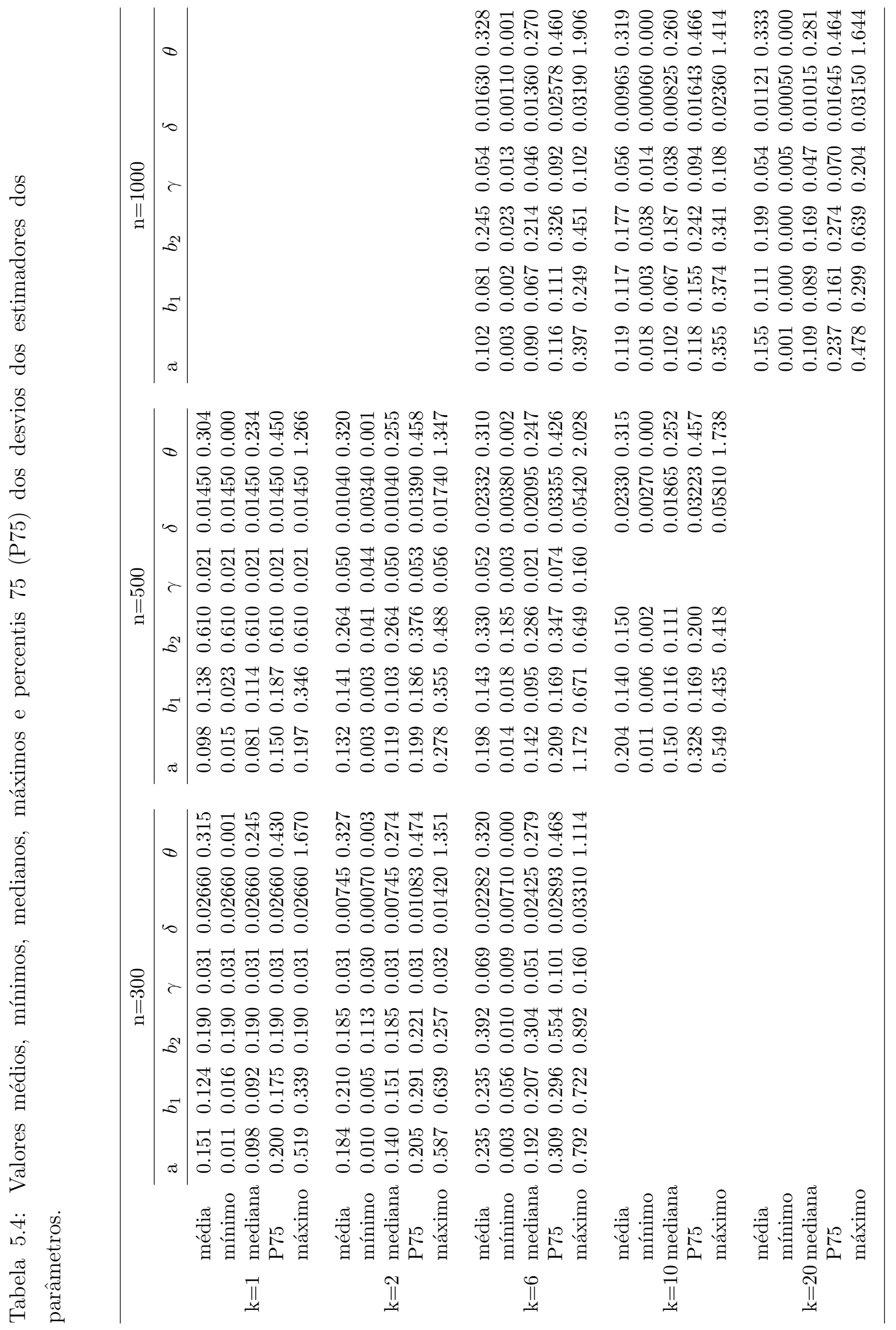




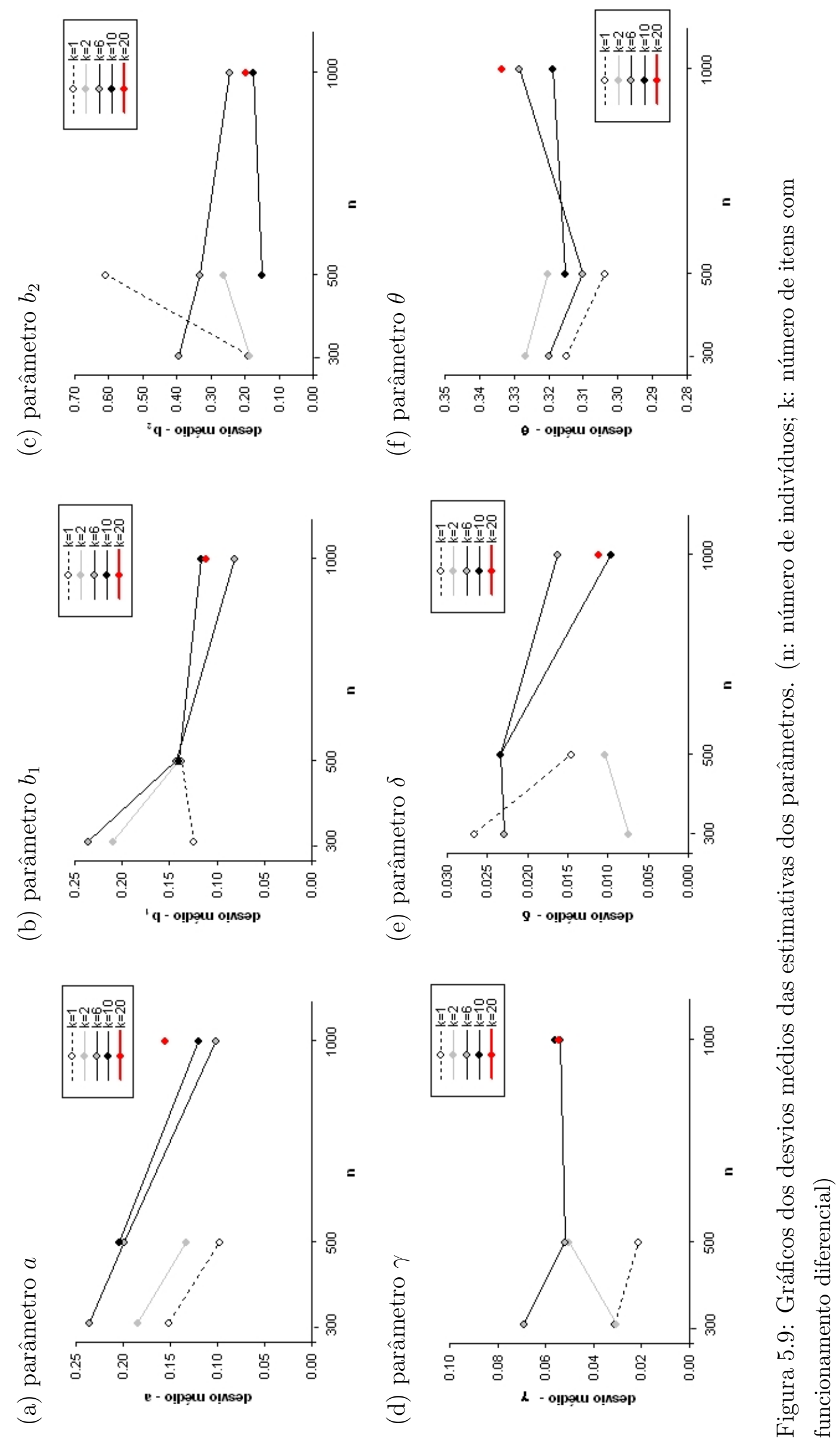




\section{Capítulo 6}

\section{Aplicação a Dados Psiquiátricos Reais}

Os dados utilizados nesta seção foram gentilmente cedidos pelo Prof. Dr. Dartiu Xavier da Silveira, do departamento de Psiquiatria da Universidade Federal de São Paulo (UNIFESP) e envolvem 348 estudantes universitários, sendo 135 mulheres (39\%) e 213 homens (61\%). Esses estudantes responderam ao questionário da Escala de Sintomas Depressivos (CES-D), apresentado no Apêndice B. O instrumento é auto-aplicável, composto por 20 itens e foi desenvolvido em 1977 com a finalidade de detectar sintomas depressivos em populações adultas. As respostas a cada um dos itens são dadas segundo a freqüência com que cada sintoma esteve presente na semana precedente à aplicação do instrumento, da seguinte forma:

\begin{tabular}{cc}
\hline Resposta & Pontuação \\
\hline raramente ou nunca & 0 \\
durante pouco tempo & 1 \\
ocasionalmente ou durante um tempo moderado & 2 \\
durante a maior parte do tempo ou todo o tempo & 3 \\
\hline
\end{tabular}

Repare que quanto maior a freqüência do sintoma, maior a pontuação no item e, portanto, maior a gravidade da depressão. Em especial para os itens 4, 8, 12 e 16, as pontuações devem ser invertidas para efeito das análises estatísticas, pois correspondem a aspectos não depressivos.

O modelo (3.3) foi aplicado aos dados para $\mathrm{i}=1, \ldots, 20, \mathrm{j}=1, \ldots, 348 ; X_{i j}$ denota uma variável dicotômica que assume os valores 1, quando o indivíduo j obtém pontuação 1, 2 ou 3 no item i, e 0 em caso contrário; $C_{i j}$ denota uma variável dicotômica que assume os 
valores 1 , se o indivíduo j é do sexo masculino e se $\mathrm{i}=17$ (referente ao item "choro"), e 0 em caso contrário; e $\theta_{j}$ representa o nível de depressão do j-ésimo indivíduo. Neste contexto, os parâmetros de item a serem estimados são: $a_{i}, b_{1 i}, b_{2,17}, \gamma_{17}$ e $\delta_{17}$. O programa WinBUGS versão 1.4.1 foi utilizado para a obtenção das respectivas estimativas da mesma forma que a descrita no capítulo anterior.

As estimativas e respectivos erros-padrão dos parâmetros de item encontram-se apresentados na Tabela 6.1 e na Figura 6.1.

Os itens mais discriminantes $\left(a_{i}\right)$ correspondem aos sintomas tristeza, felicidade, depressão e estima. Os itens menos discriminantes são aqueles relativos aos sintomas sono, valorização e apetite. Pelas estimativas de gravidade de depressão $\left(b_{1 i}\right.$ e $\left.b_{2 i}\right)$, observa-se que os sintomas com maior probabilidade de estarem presentes em baixos níveis de depressão são valorização e concentração. Os sintomas apetite, choro (apenas para o sexo masculino) e amistosidade ocorrem com probabilidade acima de $50 \%$ em níveis mais graves de depressão. Note que crises de choro são prováveis de serem observadas em níveis mais tênues de depressão nas mulheres $\left(b_{1,17}=1,06\right)$ e mais graves nos homens $\left(b_{2,17}=2,41\right)$. Adicionalmente, estima-se que a probabilidade de um homem com alto nível de depressão admitir que tem crises de choro é de $75 \%\left(\gamma_{17}=0,75\right)$. As curvas características do item (CCI) choro para os homens, para as mulheres e independentemente do gênero do indivíduo estão representadas na Figura 6.2. Este é o sintoma que mais evidencia a vantagem do modelo (3.3) sobre aqueles já existentes na literatura para itens dicotômicos. Comparando os resultados apresentados acima com os obtidos através do ajuste do ML2 ao mesmo conjunto de dados, por exemplo, as estimativas são muito parecidas e as conclusões permanecem inalteradas, a exceção do sintoma choro. No ML2, suas estimativas dos parâmetros de gravidade e discriminação são, respectivamente, 1,82 0,26 e 1,17 $\pm 0,22$. Ou seja, uma estimativa da discriminação muito próxima à do modelo (3.3) $(1,21 \pm 0,27)$, mas com apenas um valor para a estimativa de gravidade, sem distinguir a severidade de crises de choro nos homens e nas mulheres.

A distribuição de freqüências dos níveis individuais de depressão $\left(\theta_{j}\right)$ estimados encontrase representada pelo histograma na Figura 6.3 e evidencia a adequabilidade da suposição da distribuição normal dos traços latentes.

O diagnóstico de depressão costuma ser baseado na experiência clínica do médico e na pontuação total do indivíduo na CES-D. Indivíduos que obtêm pontuação menor que 16 são classificados como não depressivos, aqueles com pontuação de 16 a 23 são considerados prováveis depressivos e os que obtêm pontuação acima de 23 são classificados como deprimidos. A correlação de 0,90 do escore total da CES-D com o nível de depressão individual estimado e os boxplots apresentados na Figura 6.4, evidenciam a forte associação entre essas variáveis. Tais dados foram obtidos com 319 indivíduos que responderam a todos os 20 itens da CES-D, pois o cálculo do escore total exige que o indivíduo responda a todos os itens do questionário. Uma das vantagens do método proposto é a possibilidade de estimação do nível de depressão de indivíduos que não responderam o questionário por 


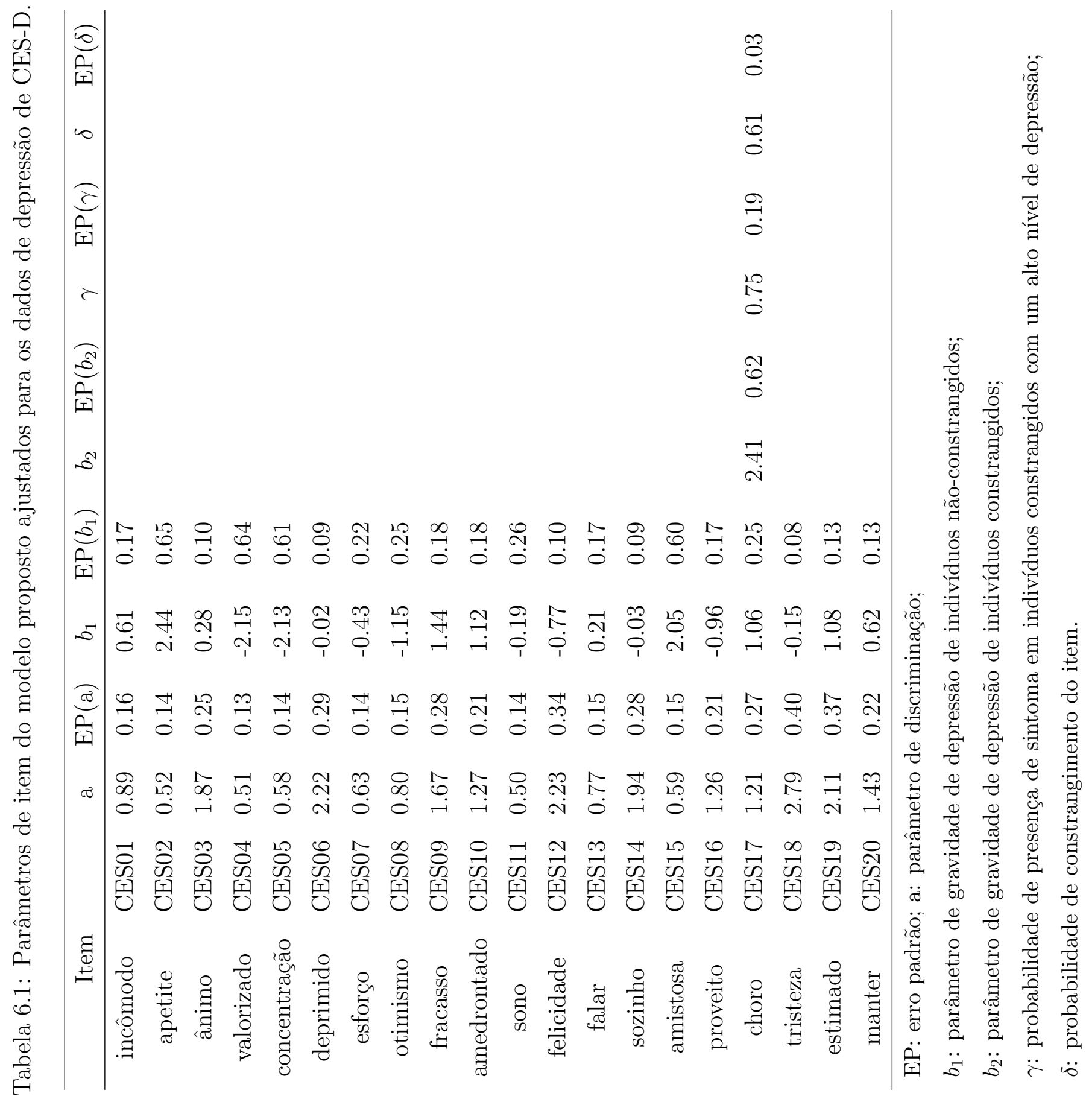


(a)

$\mathbf{a}_{\mathbf{i}}$

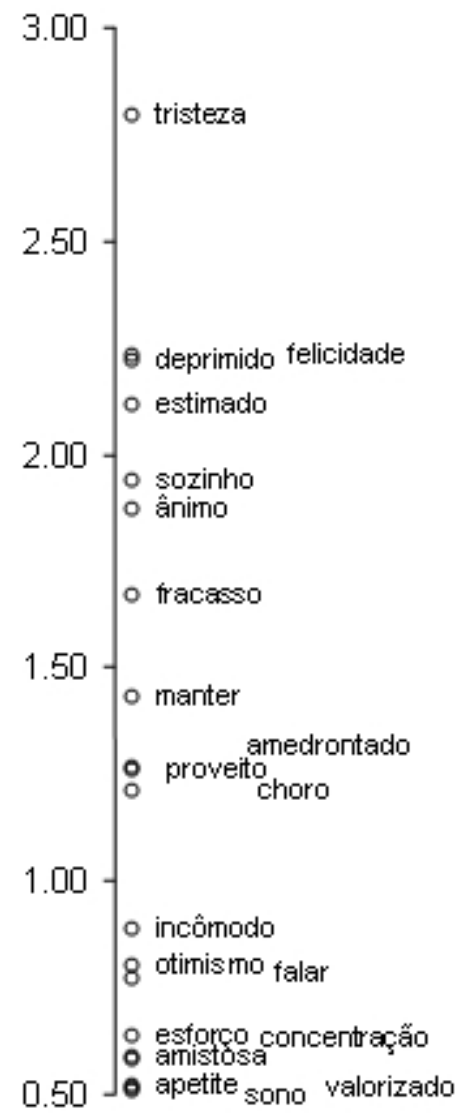

(b)

$\mathbf{b}_{1 \mathrm{i}}$ e $\mathbf{b}_{2,17}$

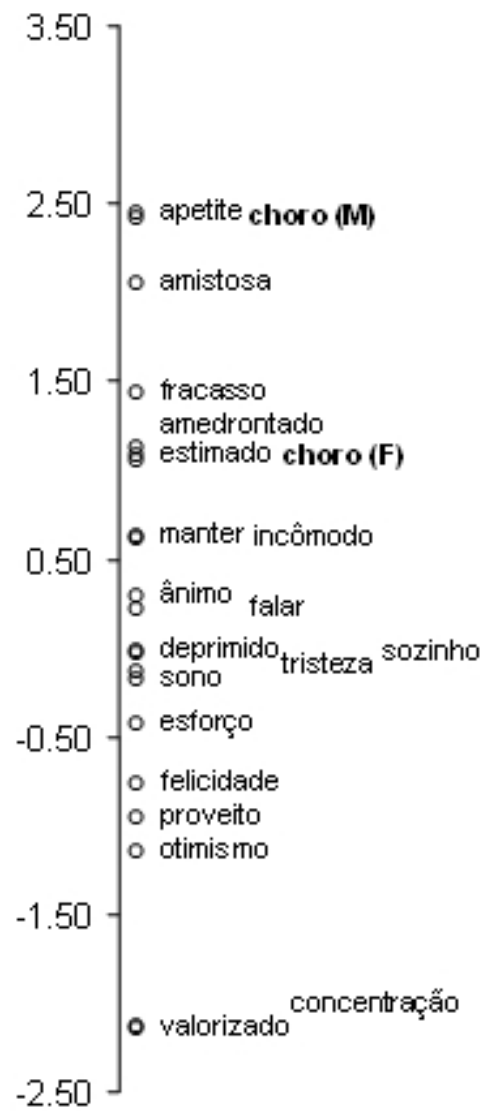

Figura 6.1: Estimativas dos parâmetros de (a) discriminação e (b) gravidade dos dados de depressão de CES-D. 


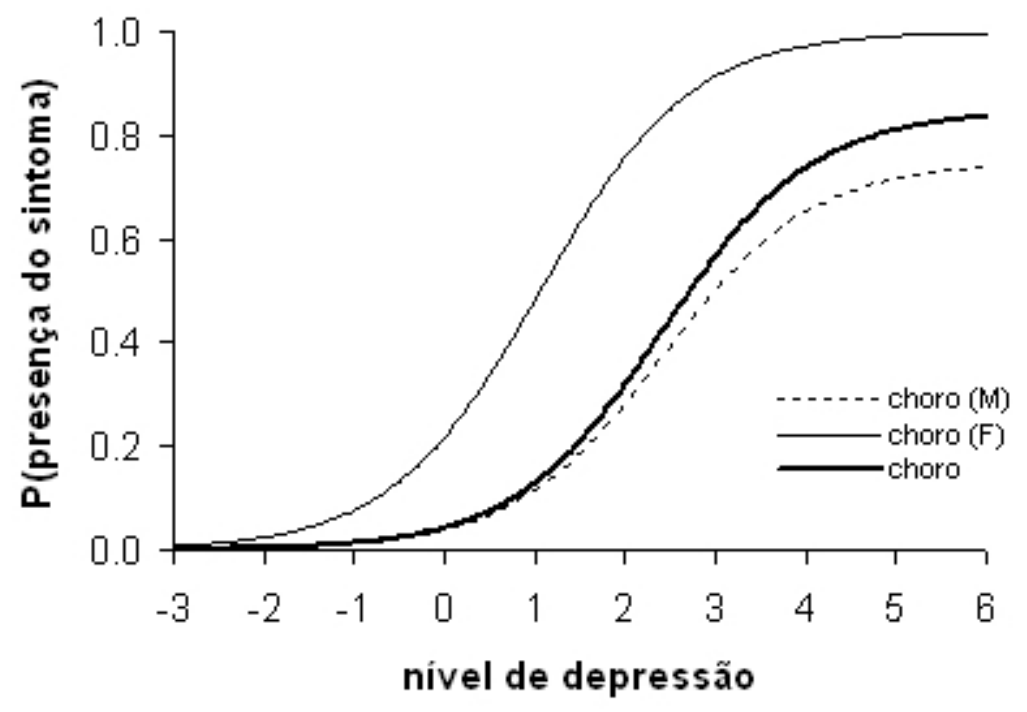

Figura 6.2: CCI choro para indivíduos do sexo masculino (M), feminino (F) e sem especificação de gênero, estimada para os dados de depressão de CES-D.

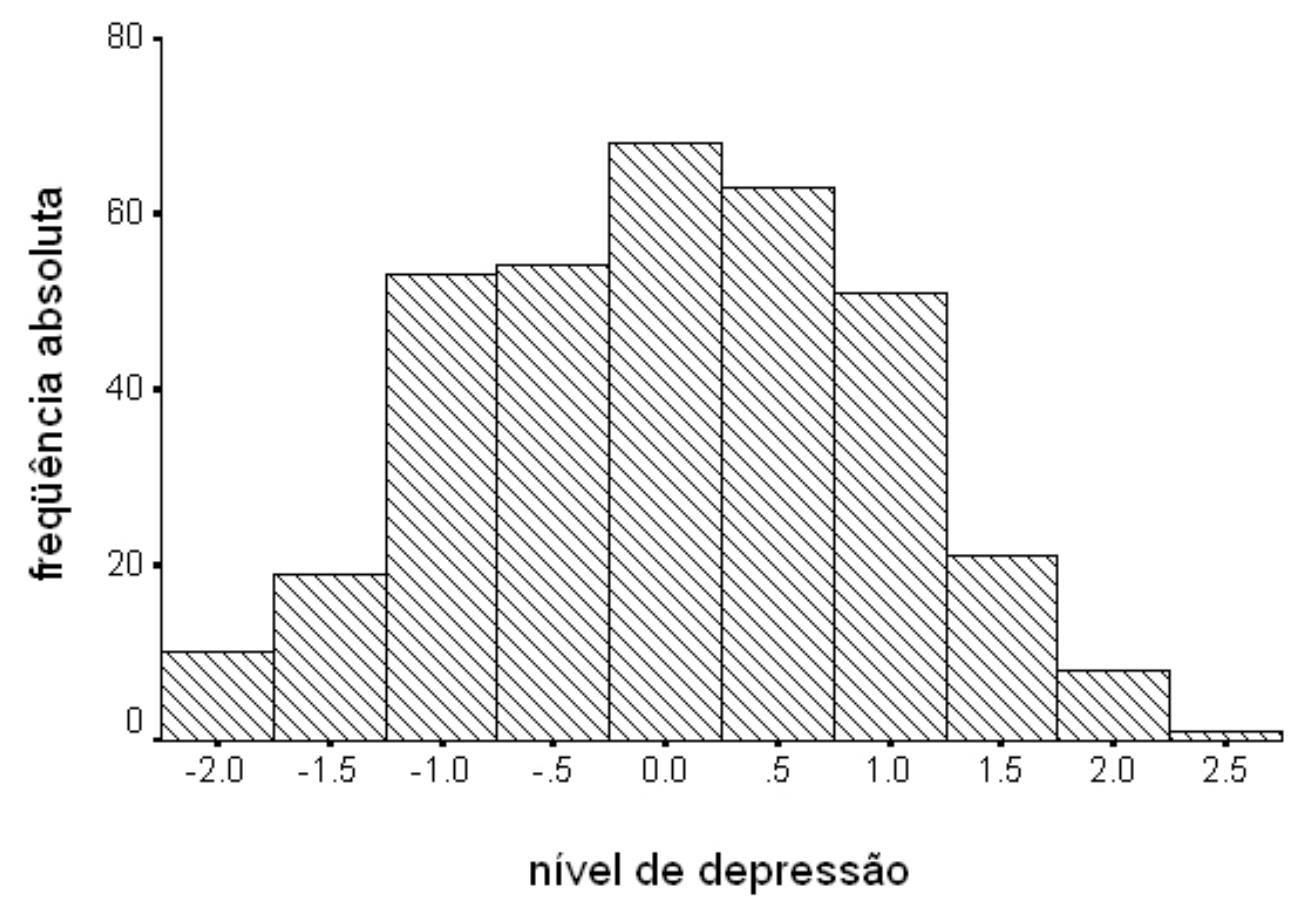

Figura 6.3: Níveis de depressão individuais estimados para os dados da CES-D. 


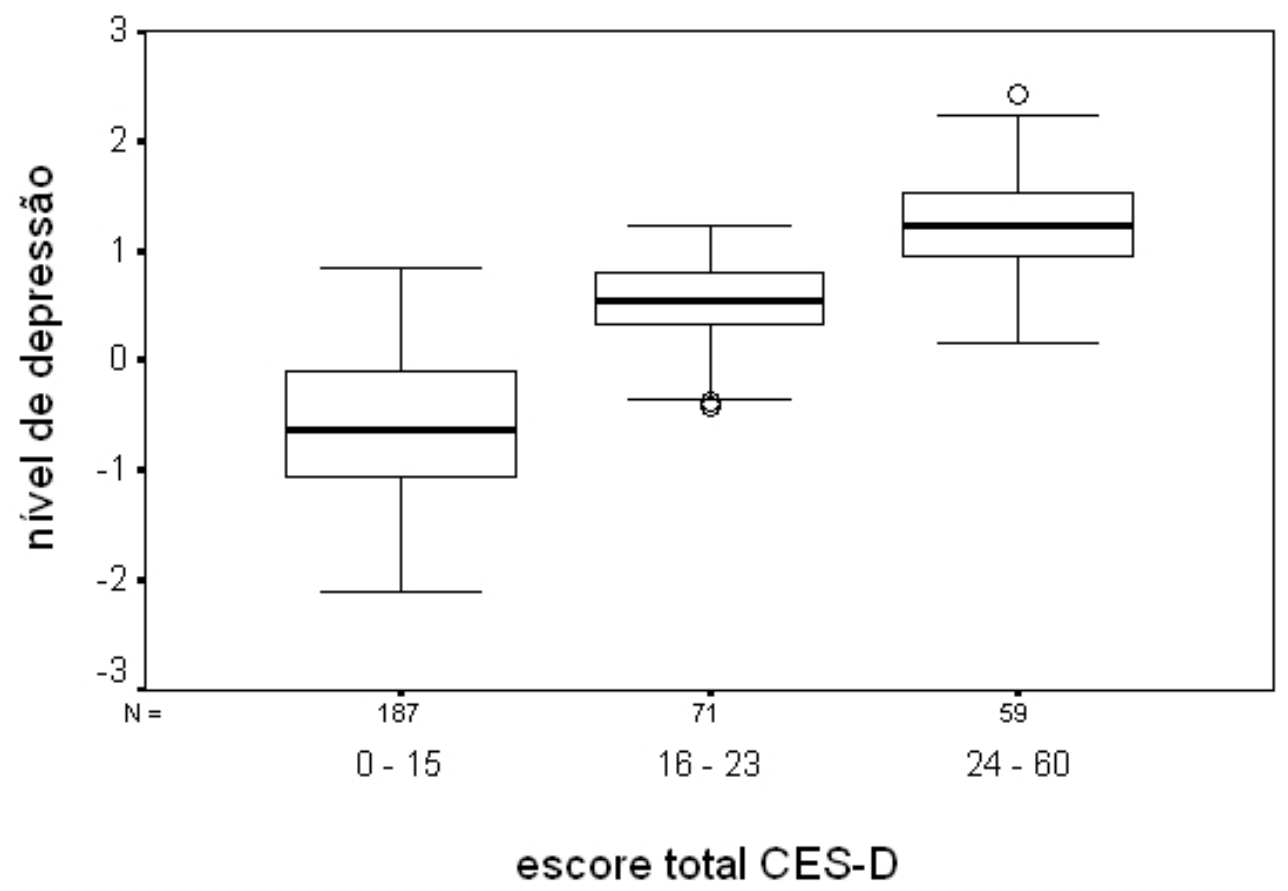

Figura 6.4: Boxplot do nível de depressão individual estimado para os diagnósticos de depressão baseados no escore total da CES-D.

completo. 


\section{Capítulo 7}

\section{Estudos Futuros}

- Métodos de Estimação

Pretende-se desenvolver expressões para a obtenção dos estimadores bayesianos dos parâmetros de itens, $\boldsymbol{\zeta}$, e de gravidade da doença, $\boldsymbol{\theta}$.

- Implementação Computacional

Para que outros pesquisadores possam aplicar a metodologia desenvolvida em problemas práticos, pretende-se aprimorar e documentar sub-rotinas para calcular os estimadores de $\boldsymbol{\zeta}$ e $\boldsymbol{\theta}$ através dos diversos métodos de estimação propostos neste trabalho (máxima verossimilhança, máxima verossimilhança marginal e bayesiano marginal). Essas sub-rotinas serão desenvolvidas no pacote R.

- Estudo de Simulação

Serão simulados em torno de 1000 conjuntos de dados, cada um deles com respostas de 500 unidades amostrais a um questionário de 20 itens. Para tanto, os parâmetros dos itens serão fixados conforme os valores descritos na Tabela 5.2 e as respostas serão geradas de acordo com o modelo probabilístico (3.3). Em cada conjunto de dados, os estimadores de $\boldsymbol{\zeta}$ e $\boldsymbol{\theta}$ serão calculados pelos diferentes métodos propostos neste trabalho. As propriedades desses estimadores serão comparadas com base nos respectivos erros quadráticos médios.

- Extensões do modelo (3.3)

O questionário da Escala de Sintomas Depressivos (CES-D), apresentado no capítulo 6 , é composto por itens com categorias de respostas ordinais. Para a aplicação do modelo (3.3), elas foram simplificadas gerando respostas dicotômicas (presença ou ausência do sintoma). Uma das possíveis extensões do modelo proposto é sua generalização para itens com respostas ordinais.

Para muitos instrumentos multidimensionais, considera-se que uma das dimensões é dominante e adotam-se os modelos unidimensionais para aplicações a dados reais. 
Nem sempre esta simplificação é adequada e pode comprometer os resultados do estudo. Um exemplo é o Inventário de Depressão de Beck (BDI). Wang et al. (2005) identificou duas dimensões que compõem o BDI: dimensão cognitivo-afetiva e dimensão somática. Neste contexto, a extensão do modelo proposto para mais de um traço latente (multidimensional) também é de bastante interesse. 


\section{Apêndice A}

\section{Expressões Detalhadas}

As expressões apresentadas neste apêndice foram obtidas por meio do software Mathematica, versão 5.2.

De 3.1 e 3.2 , tem-se

$$
\begin{gathered}
\frac{\partial P_{i j}^{*}}{\partial \theta_{j}}=\frac{-\gamma_{i}\left(-a_{i}\right) e^{-a_{i}\left(\theta_{j}-b_{2 i}\right)}}{\left(1+e^{-a_{i}\left(\theta_{j}-b_{2 i}\right)}\right)^{2}} \\
=\gamma_{i} a_{i} \frac{1}{1+e^{-a_{i}\left(\theta_{j}-b_{2 i}\right)}}\left(1-\frac{1}{1+e^{-a_{i}\left(\theta_{j}-b_{2 i}\right)}}\right)=\frac{a_{i}}{\gamma_{i}} P_{i j}^{*}\left(\gamma_{i}-P_{i j}^{*}\right) \\
\frac{\partial P_{i j}}{\partial \theta_{j}}=\frac{-e^{-a_{i}\left(\theta_{j}-b_{1 i}\right)}}{\left(1+e^{-a_{i}\left(\theta_{j}-b_{1 i}\right)}\right)^{2}}=a_{i} P_{i j}\left(1-P_{i j}\right) \\
\frac{\partial^{2} P_{i j}^{*}}{\partial \theta_{j}^{2}}=\frac{\frac{a_{i}}{\partial \theta_{j}}}{\gamma_{i}} \frac{\partial P_{i j}^{*}}{\partial \theta_{j}}\left(\gamma_{i}-P_{i j}^{*}\right)-\frac{a_{i}}{\gamma_{i}} P_{i j}^{*} \frac{\partial P_{i j}^{*}}{\partial \theta_{j}} \\
=\frac{a_{i}}{\gamma_{i}} \frac{\partial P_{i j}^{*}}{\partial \theta_{j}}\left(\gamma_{i}-P_{i j}^{*}-P_{i j}^{*}\right) \\
=\frac{a_{i}^{2}}{\gamma_{i}^{2}} P_{i j}^{*}\left(\gamma_{i}-P_{i j}^{*}\right)\left(\gamma_{i}-2 P_{i j}^{*}\right)
\end{gathered}
$$




$$
\begin{aligned}
& \frac{\partial^{2} P_{i j}}{\partial \theta_{j}^{2}}=a_{i}^{2} P_{i j}\left(1-P_{i j}\right)\left(1-2 P_{i j}\right) \\
& \frac{\partial^{2} l(\boldsymbol{\theta})}{\partial \theta_{j}^{2}}=\frac{\partial}{\partial \theta_{j}}\left[\frac{\partial l(\boldsymbol{\theta})}{\partial \theta_{j}}\right]=\frac{\partial}{\partial \theta_{j}}\left\{\sum _ { i = 1 } ^ { I } \left[\left(\frac{x_{i j} c_{i j}}{P_{i j}^{*}}-\frac{\left(1-x_{i j}\right) c_{i j}}{1-P_{i j}^{*}}\right) \frac{\partial P_{i j}^{*}}{\partial \theta_{j}}+\right.\right. \\
& \left.\left.\left(\frac{x_{i j}\left(1-c_{i j}\right)}{P_{i j}}-\frac{\left(1-x_{i j}\right)\left(1-c_{i j}\right)}{1-P_{i j}}\right) \frac{\partial P_{i j}}{\partial \theta_{j}}\right]\right\} \\
& =\sum_{i=1}^{I}\left\{\frac{\partial}{\partial \theta_{j}}\left(\frac{x_{i j} c_{i j}}{P_{i j}^{*}}-\frac{\left(1-x_{i j}\right) c_{i j}}{1-P_{i j}^{*}}\right) \frac{\partial P_{i j}^{*}}{\partial \theta_{j}}+\left(\frac{x_{i j} c_{i j}}{P_{i j}^{*}}-\frac{\left(1-x_{i j}\right) c_{i j}}{1-P_{i j}^{*}}\right) \frac{\partial^{2} P_{i j}^{*}}{\partial \theta_{j}^{2}}+\right. \\
& \frac{\partial}{\partial \theta_{j}}\left(\frac{x_{i j}\left(1-c_{i j}\right)}{P_{i j}}-\frac{\left(1-x_{i j}\right)\left(1-c_{i j}\right)}{1-P_{i j}}\right) \frac{\partial P_{i j}}{\partial \theta_{j}}+ \\
& \left.\left(\frac{x_{i j}\left(1-c_{i j}\right)}{P_{i j}}-\frac{\left(1-x_{i j}\right)\left(1-c_{i j}\right)}{1-P_{i j}}\right) \frac{\partial^{2} P_{i j}}{\partial \theta_{j}^{2}}\right\} \\
& =\sum_{i=1}^{I}\left\{\left[-\frac{x_{i j} c_{i j}}{\left(P_{i j}^{*}\right)^{2}} \frac{\partial P_{i j}^{*}}{\partial \theta_{j}}-\frac{\left(1-x_{i j}\right) c_{i j}}{\left(1-P_{i j}^{*}\right)^{2}} \frac{\partial P_{i j}^{*}}{\partial \theta_{j}}\right] \frac{\partial P_{i j}^{*}}{\partial \theta_{j}}+\left[\frac{x_{i j} c_{i j}}{P_{i j}^{*}}-\frac{\left(1-x_{i j}\right) c_{i j}}{1-P_{i j}^{*}}\right] \frac{\partial^{2} P_{i j}^{*}}{\partial \theta_{j}^{2}}+\right. \\
& {\left[-\frac{x_{i j}\left(1-c_{i j}\right)}{P_{i j}^{2}} \frac{\partial P_{i j}}{\partial \theta_{j}}-\frac{\left(1-x_{i j}\right)\left(1-c_{i j}\right)}{\left(1-P_{i j}\right)^{2}} \frac{\partial P_{i j}}{\partial \theta_{j}}\right] \frac{\partial P_{i j}}{\partial \theta_{j}}+} \\
& \left.\left[\frac{x_{i j}\left(1-c_{i j}\right)}{P_{i j}}-\frac{\left(1-x_{i j}\right)\left(1-c_{i j}\right)}{1-P_{i j}}\right] \frac{\partial^{2} P_{i j}}{\partial \theta_{j}^{2}}\right\} \\
& =\sum_{i=1}^{I}\left\{\left[-\frac{x_{i j} c_{i j}}{\left(P_{i j}^{*}\right)^{2}}-\frac{\left(1-x_{i j}\right) c_{i j}}{\left(1-P_{i j}^{*}\right)^{2}}\right]\left(\frac{\partial P_{i j}^{*}}{\partial \theta_{j}}\right)^{2}+\right. \\
& {\left[\frac{x_{i j} c_{i j}-x_{i j} c_{i j} P_{i j}^{*}-c_{i j} P_{i j}^{*}+x_{i j} c_{i j} P_{i j}^{*}}{P_{i j}^{*}\left(1-P_{i j}^{*}\right)}\right] \frac{\partial^{2} P_{i j}^{*}}{\partial \theta_{j}^{2}}+} \\
& {\left[-\frac{x_{i j}\left(1-c_{i j}\right)}{P_{i j}^{2}}-\frac{\left(1-x_{i j}\right)\left(1-c_{i j}\right)}{\left(1-P_{i j}\right)^{2}}\right]\left(\frac{\partial P_{i j}}{\partial \theta_{j}}\right)^{2}+} \\
& \left.\left[\frac{x_{i j}-x_{i j} c_{i j}-x_{i j} P_{i j}+x_{i j} c_{i j} P_{i j}-P_{i j}+c_{i j} P_{i j}+x_{i j} P_{i j}-x_{i j} c_{i j} P_{i j}}{P_{i j}\left(1-P_{i j}\right)}\right] \frac{\partial^{2} P_{i j}}{\partial \theta_{j}^{2}}\right\} \\
& =\sum_{i=1}^{I}\left\{\left[\frac{-x_{i j} c_{i j}+2 x_{i j} c_{i j} P_{i j}^{*}-x_{i j} c_{i j}\left(P_{i j}^{*}\right)^{2}-c_{i j}\left(P_{i j}^{*}\right)^{2}+x_{i j} c_{i j}\left(P_{i j}^{*}\right)^{2}}{\left(P_{i j}^{*}\right)^{2}\left(1-P_{i j}^{*}\right)^{2}}\right]\left(\frac{\partial P_{i j}^{*}}{\partial \theta_{j}}\right)^{2}+\right.
\end{aligned}
$$




$$
\begin{aligned}
& {\left[\frac{c_{i j}\left(x_{i j}-P_{i j}^{*}\right)}{P_{i j}^{*}\left(1-P_{i j}^{*}\right)}\right] \frac{\partial^{2} P_{i j}^{*}}{\partial \theta_{j}^{2}}+\left[\left(-x_{i j}+2 x_{i j} P_{i j}-x_{i j} P_{i j}^{2}+x_{i j} c_{i j}-2 x_{i j} c_{i j} P_{i j}+\right.\right.} \\
& \left.\left.x_{i j} c_{i j} P_{i j}^{2}-P_{i j}^{2}+c_{i j} P_{i j}^{2}+x_{i j} P_{i j}^{2}-x_{i j} c_{i j} P_{i j}^{2}\right) \frac{1}{P_{i j}^{2}\left(1-P_{i j}\right)^{2}}\right]\left(\frac{\partial P_{i j}}{\partial \theta_{j}}\right)^{2}+ \\
& \left.\left[\frac{\left(1-c_{i j}\right)\left(x_{i j}-P_{i j}\right)}{P_{i j}\left(1-P_{i j}\right)}\right] \frac{\partial^{2} P_{i j}}{\partial \theta_{j}^{2}}\right\} \\
& =\sum_{i=1}^{I}\left\{\frac{-c_{i j}\left(x_{i j}-2 x_{i j} P_{i j}^{*}+\left(P_{i j}^{*}\right)^{2}\right)}{\left(P_{i j}^{*}\right)^{2}\left(1-P_{i j}^{*}\right)^{2}}\left(\frac{\partial P_{i j}^{*}}{\partial \theta_{j}}\right)^{2}\left[\frac{c_{i j}\left(x_{i j}-P_{i j}^{*}\right)}{P_{i j}^{*}\left(1-P_{i j}^{*}\right)}\right] \frac{\partial^{2} P_{i j}^{*}}{\partial \theta_{j}^{2}}+\right. \\
& \left.\left[\frac{-\left(1-c_{i j}\right)\left(x_{i j}-2 x_{i j} P_{i j}+P_{i j}^{2}\right)}{P_{i j}^{2}\left(1-P_{i j}\right)^{2}}\right]\left(\frac{\partial P_{i j}}{\partial \theta_{j}}\right)^{2}+\left[\frac{\left(1-c_{i j}\right)\left(x_{i j}-P_{i j}\right)}{P_{i j}\left(1-P_{i j}\right)}\right] \frac{\partial^{2} P_{i j}}{\partial \theta_{j}^{2}}\right\} \\
& =\sum_{i=1}^{I}\left\{\frac{-c_{i j}\left(x_{i j}-2 x_{i j} P_{i j}^{*}+\left(P_{i j}^{*}\right)^{2}\right)}{\left(P_{i j}^{*}\right)^{2}\left(1-P_{i j}^{*}\right)^{2}} \frac{a_{i}^{2}}{\gamma_{i}^{2}}\left(P_{i j}^{*}\right)^{2}\left(\gamma_{i}-P_{i j}^{*}\right)^{2}+\right. \\
& \frac{c_{i j}\left(x_{i j}-P_{i j}^{*}\right)}{P_{i j}^{*}\left(1-P_{i j}^{*}\right)} \frac{a_{i}^{2}}{\gamma_{i}^{2}} P_{i j}^{*}\left(\gamma_{i}-P_{i j}^{*}\right)\left(\gamma_{i}-2 P_{i j}^{*}\right)+ \\
& \frac{-\left(1-c_{i j}\right)\left(x_{i j}-2 x_{i j} P_{i j}+P_{i j}^{2}\right)}{P_{i j}^{2}\left(1-P_{i j}\right)^{2}} a_{i}^{2} P_{i j}^{2}\left(1-P_{i j}\right)^{2}+ \\
& \left.\frac{\left(1-c_{i j}\right)\left(x_{i j}-P_{i j}\right)}{P_{i j}\left(1-P_{i j}\right)} a_{i}^{2} P_{i j}\left(1-P_{i j}\right)\left(1-2 P_{i j}\right)\right\} \\
& =\sum_{i=1}^{I}\left\{\frac{a_{i}^{2}}{\gamma_{i}^{2}} \frac{\left(\gamma_{i}-P_{i j}^{*}\right)}{\left(1-P_{i j}^{*}\right)} c_{i j}\left[-\frac{\left(\gamma_{i}-P_{i j}^{*}\right)\left(x_{i j}-2 x_{i j} P_{i j}^{*}+\left(P_{i j}^{*}\right)^{2}\right)}{\left(1-P_{i j}^{*}\right)}+\left(x_{i j}-P_{i j}^{*}\right)\left(\gamma_{i}-2 P_{i j}^{*}\right)\right]\right. \\
& \left.a_{i}^{2}\left(1-c_{i j}\right)\left[-\left(x_{i j}-2 x_{i j} P_{i j}+P_{i j}^{2}\right)+\left(x_{i j}-P_{i j}\right)\left(1-2 P_{i j}\right)\right]\right\} \\
& =\sum_{i=1}^{I}\left\{\frac{a_{i}^{2}}{\gamma_{i}^{2}} \frac{\left(\gamma_{i}-P_{i j}^{*}\right)}{\left(1-P_{i j}^{*}\right)} c_{i j}\left(x_{i j}-P_{i j}^{*}\right)\left[-\frac{\left(\gamma_{i}-P_{i j}^{*}\right)\left(x_{i j}-P_{i j}^{*}\right)}{\left(1-P_{i j}^{*}\right)}+\left(\gamma_{i}-2 P_{i j}^{*}\right)\right]\right. \\
& \left.a_{i}^{2}\left(1-c_{i j}\right)\left(x_{i j}-P_{i j}\right)\left[-\left(x_{i j}-P_{i j}\right)+\left(1-2 P_{i j}\right)\right]\right\} \\
& =\sum_{i=1}^{I}\left\{\frac { a _ { i } ^ { 2 } } { \gamma _ { i } ^ { 2 } } \frac { ( \gamma _ { i } - P _ { i j } ^ { * } ) } { ( 1 - P _ { i j } ^ { * } ) } c _ { i j } ( x _ { i j } - P _ { i j } ^ { * } ) \left[\left(-x_{i j} \gamma_{i}+x_{i j} P_{i j}^{*}+\gamma_{i} P_{i j}^{*}-\left(P_{i j}^{*}\right)^{2}+\gamma_{i}-\gamma_{i} P_{i j}^{*}\right.\right.\right. \\
& \left.\left.\left.-2 P_{i j}^{*}+2\left(P_{i j}^{*}\right)^{2}\right) \frac{1}{\left(1-P_{i j}^{*}\right)}\right]+a_{i}^{2}\left(1-c_{i j}\right)\left(x_{i j}-P_{i j}\right)\left(1-x_{i j}-P_{i j}\right)\right\}
\end{aligned}
$$




$$
\begin{aligned}
= & \sum_{i=1}^{I}\left\{\frac{a_{i}^{2}}{\gamma_{i}^{2}} \frac{\left(\gamma_{i}-P_{i j}^{*}\right)}{\left(1-P_{i j}^{*}\right)}\left(x_{i j}-P_{i j}^{*}\right) c_{i j}\left[\frac{\left(\gamma_{i}-P_{i j}^{*}\right)\left(1-x_{i j}\right)}{1-P_{i j}^{*}}-P_{i j}^{*}\right]+\right. \\
& \left.+a_{i}^{2}\left(1-c_{i j}\right)\left(x_{i j}-P_{i j}\right)\left(1-x_{i j}-P_{i j}\right)\right\}
\end{aligned}
$$

Em que a terceira última igualdade de (A.6), deriva do fato de $x_{i j}=x_{i j}^{2}$, quando $x_{i j}=0$ ou 1 .

$$
\begin{aligned}
& \frac{\partial P\left(X_{i j}=x_{i j}, C_{i j}=c_{i j} \mid \theta, \boldsymbol{\zeta}\right)}{\partial \boldsymbol{\zeta}_{i}}= \\
& =\frac{\partial}{\partial \boldsymbol{\zeta}_{i}}\left\{\left[\left(P_{i j}^{*}\right)^{x_{i j}}\left(1-P_{i j}^{*}\right)^{\left(1-x_{i j}\right)} \delta_{i}\right]^{c_{i j}}\left[P_{i j}^{x_{i j}}\left(1-P_{i j}\right)^{\left(1-x_{i j}\right)}\left(1-\delta_{i}\right)\right]^{1-c_{i j}}\right\} \\
& =\frac{\partial}{\partial \boldsymbol{\zeta}_{i}}\left[\left(P_{i j}^{*}\right)^{x_{i j} c_{i j}}\left(1-P_{i j}^{*}\right)^{\left(1-x_{i j}\right) c_{i j}} \delta_{i}^{c_{i j}} P_{i j}^{x_{i j}\left(1-c_{i j}\right)}\left(1-P_{i j}\right)^{\left(1-x_{i j}\right)\left(1-c_{i j}\right)}\left(1-\delta_{i}\right)^{\left(1-c_{i j}\right)}\right] \\
& =x_{i j} c_{i j}\left(P_{i j}^{*}\right)^{x_{i j} c_{i j}-1} \frac{\partial P_{i j}^{*}}{\partial \boldsymbol{\zeta}_{i}}\left(1-P_{i j}^{*}\right)^{\left(1-x_{i j}\right) c_{i j}} \delta_{i}^{c_{i j}} P_{i j}^{x_{i j}\left(1-c_{i j}\right)}\left(1-P_{i j}\right)^{\left(1-x_{i j}\right)\left(1-c_{i j}\right)}\left(1-\delta_{i}\right)^{\left(1-c_{i j}\right)}- \\
& \left(P_{i j}^{*}\right)^{x_{i j} c_{i j}}\left(1-x_{i j}\right) c_{i j}\left(1-P_{i j}^{*}\right)^{\left(1-x_{i j}\right) c_{i j}-1} \frac{\partial P_{i j}^{*}}{\partial \zeta_{i}} \delta_{i}^{c_{i j}} P_{i j}^{x_{i j}\left(1-c_{i j}\right)}\left(1-P_{i j}\right)^{\left(1-x_{i j}\right)\left(1-c_{i j}\right)}\left(1-\delta_{i}\right)^{\left(1-c_{i j}\right)}+ \\
& \left(P_{i j}^{*}\right)^{x_{i j} c_{i j}}\left(1-P_{i j}^{*}\right)^{\left(1-x_{i j}\right) c_{i j}} c_{i j} \delta_{i}^{c_{i j}-1} \frac{\partial \delta_{i}}{\partial \boldsymbol{\zeta}_{i}} P_{i j}^{x_{i j}\left(1-c_{i j}\right)}\left(1-P_{i j}\right)^{\left(1-x_{i j}\right)\left(1-c_{i j}\right)}\left(1-\delta_{i}\right)^{\left(1-c_{i j}\right)}+ \\
& \left(P_{i j}^{*}\right)^{x_{i j} c_{i j}}\left(1-P_{i j}^{*}\right)^{\left(1-x_{i j}\right) c_{i j}} \delta_{i}^{c_{i j}} x_{i j}\left(1-c_{i j}\right) P_{i j}^{x_{i j}\left(1-c_{i j}\right)-1} \frac{\partial P_{i j}}{\partial \zeta_{i}}\left(1-P_{i j}\right)^{\left(1-x_{i j}\right)\left(1-c_{i j}\right)}\left(1-\delta_{i}\right)^{\left(1-c_{i j}\right)}- \\
& \left(P_{i j}^{*}\right)^{x_{i j} c_{i j}}\left(1-P_{i j}^{*}\right)^{\left(1-x_{i j}\right) c_{i j}} \delta_{i}^{c_{i j}} P_{i j}^{x_{i j}\left(1-c_{i j}\right)}\left(1-x_{i j}\right)\left(1-c_{i j}\right)\left(1-P_{i j}\right)^{\left(1-x_{i j}\right)\left(1-c_{i j}\right)-1} \frac{\partial P_{i j}}{\partial \boldsymbol{\zeta}_{i}}\left(1-\delta_{i}\right)^{\left(1-c_{i j}\right)}- \\
& \left(P_{i j}^{*}\right)^{x_{i j} c_{i j}}\left(1-P_{i j}^{*}\right)^{\left(1-x_{i j}\right) c_{i j}} \delta_{i}^{c_{i j}} P_{i j}^{x_{i j}\left(1-c_{i j}\right)}\left(1-P_{i j}\right)^{\left(1-x_{i j}\right)\left(1-c_{i j}\right)}\left(1-c_{i j}\right)\left(1-\delta_{i}\right)^{\left(-c_{i j}\right)} \frac{\partial \delta_{i}}{\partial \boldsymbol{\zeta}_{i}} \\
& =P\left(X_{i j}=x_{i j}, C_{i j}=c_{i j} \mid \theta, \boldsymbol{\zeta}\right)\left[\frac{\partial P_{i j}^{*}}{\partial \boldsymbol{\zeta}_{i}} \frac{x_{i j} c_{i j}}{P_{i j}^{*}}-\frac{\partial P_{i j}^{*}}{\partial \boldsymbol{\zeta}_{i}} \frac{\left(1-x_{i j}\right) c_{i j}}{\left(1-P_{i j}^{*}\right)}+\frac{\partial \delta_{i}}{\partial \boldsymbol{\zeta}_{i}} \frac{c_{i j}}{\delta_{i}}+\right. \\
& \left.\frac{\partial P_{i j}}{\partial \boldsymbol{\zeta}_{i}} \frac{x_{i j}\left(1-c_{i j}\right)}{P_{i j}}-\frac{\partial P_{i j}}{\partial \boldsymbol{\zeta}_{i}} \frac{\left(1-x_{i j}\right)\left(1-c_{i j}\right)}{\left(1-P_{i j}\right)}-\frac{\partial \delta_{i}}{\partial \boldsymbol{\zeta}_{i}} \frac{\left(1-c_{i j}\right)}{\left(1-\delta_{i}\right)}\right] \\
& =P\left(X_{i j}=x_{i j}, C_{i j}=c_{i j} \mid \theta, \zeta\right)\left[\frac{\partial P_{i j}^{*}}{\partial \boldsymbol{\zeta}_{i}} \frac{c_{i j}\left(x_{i j}-P_{i j}^{*}\right)}{P_{i j}^{*}\left(1-P_{i j}^{*}\right)}+\frac{\partial \delta_{i}}{\partial \boldsymbol{\zeta}_{i}} \frac{\left(c_{i j}-\delta_{i}\right)}{\delta_{i}\left(1-\delta_{i}\right)}+\frac{\partial P_{i j}}{\partial \boldsymbol{\zeta}_{i}} \frac{\left(1-c_{i j}\right)\left(x_{i j}-P_{i j}\right)}{P_{i j}\left(1-P_{i j}\right)}\right]
\end{aligned}
$$




$$
\begin{aligned}
& \frac{\partial P_{i j}^{*}}{\partial a_{i}}=\frac{\gamma_{i}\left(\theta_{j}-b_{2 i}\right) e^{-a_{i}\left(\theta_{j}-b_{2 i}\right)}}{\left(1+e^{-a_{i}\left(\theta_{j}-b_{2 i}\right)}\right)^{2}} \\
& =\gamma_{i}\left(\theta_{j}-b_{2 i}\right) \frac{1}{1+e^{-a_{i}\left(\theta_{j}-b_{2 i}\right)}}\left(1-\frac{1}{1+e^{-a_{i}\left(\theta_{j}-b_{2 i}\right)}}\right) \\
& =\left(\theta_{j}-b_{2 i}\right) P_{i j}^{*}\left(1-\frac{P_{i j}^{*}}{\gamma_{i}}\right) \\
& =\frac{\left(\theta_{j}-b_{2 i}\right)}{\gamma_{i}} P_{i j}^{*}\left(\gamma_{i}-P_{i j}^{*}\right) \\
& \frac{\partial P_{i j}^{*}}{\partial b_{2 i}}=\frac{-\gamma_{i} a_{i} e^{-a_{i}\left(\theta_{j}-b_{2 i}\right)}}{\left(1+e^{-a_{i}\left(\theta_{j}-b_{2 i}\right)}\right)^{2}} \\
& =-\gamma_{i} a_{i} \frac{1}{1+e^{-a_{i}\left(\theta_{j}-b_{2 i}\right)}}\left(1-\frac{1}{1+e^{-a_{i}\left(\theta_{j}-b_{2 i}\right)}}\right) \\
& =-a_{i} P_{i j}^{*}\left(1-\frac{P_{i j}^{*}}{\gamma_{i}}\right) \\
& =-\frac{a_{i}}{\gamma_{i}} P_{i j}^{*}\left(\gamma_{i}-P_{i j}^{*}\right) \\
& \frac{\partial P_{i j}^{*}}{\partial \gamma_{i}}=\frac{1+e^{-a_{i}\left(\theta_{j}-b_{2 i}\right)}}{\left(1+e^{-a_{i}\left(\theta_{j}-b_{2 i}\right)}\right)^{2}} \\
& =\frac{P_{i j}^{*}}{\gamma_{i}} \\
& \frac{\partial P_{i j}^{*}}{\partial b_{1 i}}=\frac{\partial P_{i j}^{*}}{\partial \delta_{i}}=0 \\
& \frac{\partial P_{i j}}{\partial a_{i}}=\left(\theta_{j}-b_{1 i}\right) P_{i j}\left(1-P_{i j}\right) \\
& \frac{\partial P_{i j}}{\partial b_{1 i}}=-a_{i} P_{i j}\left(1-P_{i j}\right) \\
& \frac{\partial P_{i j}}{\partial b_{2 i}}=\frac{\partial P_{i j}}{\partial \delta_{i}}=\frac{\partial P_{i j}}{\partial \gamma_{i}}=0
\end{aligned}
$$




$$
\begin{gathered}
\frac{\partial \delta_{i}}{\partial \delta_{i}}=1 \\
\frac{\partial \delta_{i}}{\partial a_{i}}=\frac{\partial \delta_{i}}{\partial b_{1 i}}=\frac{\partial \delta_{i}}{\partial b_{2 i}}=\frac{\partial \delta_{i}}{\partial \gamma_{i}}=0 \\
\frac{\partial}{\partial \boldsymbol{\zeta}_{i}}\left[\frac{c_{i j}\left(x_{i j}-P_{i j}^{*}\right)}{P_{i j}^{*}\left(1-P_{i j}^{*}\right)}\right]=\frac{\left[-c_{i j} P_{i j}^{*}\left(1-P_{i j}^{*}\right)-c_{i j}\left(x_{i j}-P_{i j}^{*}\right)\left(1-2 P_{i j}^{*}\right)\right] \frac{\partial P_{i j}^{*}}{\partial \boldsymbol{\zeta}_{i}}}{\left[-c_{i j} P_{i j}^{*}+c_{i j}\left(P_{i j}^{*}\right)^{2}-c_{i j} x_{i j}+2 c_{i j} x_{i j} P_{i j}^{*}+c_{i j} P_{i j}^{*}-2 c_{i j}\left(P_{i j}^{*}\right)^{2}\right] \frac{\partial P_{i j}^{*}}{\partial \boldsymbol{\zeta}_{i}}} \\
=\frac{-c_{i j} \frac{\partial P_{i j}^{*}}{\partial \boldsymbol{\zeta}_{i}}\left[x_{i j}^{*}-2 x_{i j} P_{i j}^{*}+\left(1-P_{i j}^{*}\right)\right]^{2}}{\left.\left[P_{i j}^{*}\left(1-P_{i j}^{*}\right)\right]^{2}\right]} \\
=\frac{\partial P_{i j}^{*}}{\partial \boldsymbol{\zeta}_{i}}\left(\frac{x_{i j}-P_{i j}^{*}}{P_{i j}^{*}\left(1-P_{i j}^{*}\right)}\right)^{2}
\end{gathered}
$$

Para a última igualdade de A.17, note que $x_{i j}=x_{i j}^{2}$, quando $x_{i j}=0$ ou 1 .

$$
\begin{aligned}
\frac{\partial}{\partial \boldsymbol{\zeta}_{i}}\left[\frac{c_{i j}-\delta_{i}}{\delta_{i}\left(1-\delta_{i}\right)}\right] & =\frac{\left[-\delta_{i}\left(1-\delta_{i}\right)-\left(c_{i j}-\delta_{i}\right)\left(1-2 \delta_{i}\right)\right] \frac{\partial \delta_{i}}{\partial \boldsymbol{\zeta}_{i}}}{\left[\delta_{i}\left(1-\delta_{i}\right)\right]^{2}} \\
& =\frac{\left[-\delta_{i}+\delta_{i}^{2}-c_{i j}+2 c_{i j} \delta_{i}+\delta_{i}-2 \delta_{i}^{2}\right] \frac{\partial \delta_{i}}{\partial \boldsymbol{\zeta}_{i}}}{\left[\delta_{i}\left(1-\delta_{i}\right)\right]^{2}} \\
& =\frac{-\left[c_{i j}-2 c_{i j} \delta_{i}+\delta_{i}^{2}\right] \frac{\partial \delta_{i}}{\partial \boldsymbol{\zeta}_{i}}}{\left[\delta_{i}\left(1-\delta_{i}\right)\right]^{2}} \\
& =-\frac{\partial \delta_{i}}{\partial \boldsymbol{\zeta}_{i}}\left[\frac{c_{i j}-\delta_{i}}{\delta_{i}\left(1-\delta_{i}\right)}\right]^{2}
\end{aligned}
$$

Para a última igualdade de A.18, note que $c_{i j}=c_{i j}^{2}$, quando $c_{i j}=0$ ou 1 . 


$$
\begin{aligned}
& \frac{\partial}{\partial \boldsymbol{\zeta}_{i}}\left[\frac{\left(1-c_{i j}\right)\left(1-x_{i j}-P_{i j}\right)}{P_{i j}\left(1-P_{i j}\right)}\right]=\frac{\left[-\left(1-c_{i j}\right) P_{i j}\left(1-P_{i j}\right)-\left(1-c_{i j}\right)\left(x_{i j}-P_{i j}\right)\left(1-2 P_{i j}\right)\right] \frac{\partial P_{i j}}{\partial \boldsymbol{\zeta}_{i}}}{\left[P_{i j}\left(1-P_{i j}\right)\right]^{2}} \\
& =\frac{\left[-P_{i j}+P_{i j}^{2}+c_{i j} P_{i j}-c_{i j} P_{i j}^{2}-x_{i j}+2 x_{i j} P_{i j}+P_{i j}-2 P_{i j}^{2}+c_{i j} x_{i j}-2 c_{i j} x_{i j} P_{i j}-c_{i j} P_{i j}+2 c_{i j} P_{i j}^{2}\right] \frac{\partial P_{i j}}{\partial \boldsymbol{\zeta}_{i}}}{\left[P_{i j}\left(1-P_{i j}\right)\right]^{2}} \\
& =\frac{\left[-\left(x_{i j}-P_{i j}\right)^{2}+c_{i j}\left(x_{i j}-P_{i j}\right)^{2}\right] \frac{\partial P_{i j}}{\partial \boldsymbol{\zeta}_{i}}}{\left[P_{i j}\left(1-P_{i j}\right)\right]^{2}} \\
& =-\frac{\partial P_{i j}}{\partial \boldsymbol{\zeta}_{i}}\left(1-c_{i j}\right)\left[\frac{x_{i j}-P_{i j}}{P_{i j}\left(1-P_{i j}\right)}\right]^{2}
\end{aligned}
$$

A penúltima igualdade de A.19, deve-se a $x_{i j}=x_{i j}^{2}$, quando $x_{i j}=0$ ou 1 .

$$
\begin{aligned}
& \frac{\partial}{\partial \boldsymbol{\zeta}_{i}}\left\{\left[\left(\frac{\partial P_{i j}^{*}}{\partial \boldsymbol{\zeta}_{i}}\right)^{t} \frac{c_{i j}\left(x_{i j}-P_{i j}^{*}\right)}{P_{i j}^{*}\left(1-P_{i j}^{*}\right)}+\left(\frac{\partial \delta_{i}}{\partial \boldsymbol{\zeta}_{i}}\right)^{t} \frac{\left(c_{i j}-\delta_{i}\right)}{\delta_{i}\left(1-\delta_{i}\right)}+\right.\right. \\
& \left.\left.\left(\frac{\partial P_{i j}}{\partial \boldsymbol{\zeta}_{i}}\right)^{t} \frac{\left(1-c_{i j}\right)\left(x_{i j}-P_{i j}\right)}{P_{i j}\left(1-P_{i j}\right)}\right] P\left(\boldsymbol{x}_{\cdot j}, \boldsymbol{c}_{\cdot j} \mid \theta, \boldsymbol{\zeta}\right)\right\} \\
& =\frac{\partial}{\partial \boldsymbol{\zeta}_{i}}\left[\left(\frac{\partial P_{i j}^{*}}{\partial \boldsymbol{\zeta}_{i}}\right)^{t} \frac{c_{i j}\left(x_{i j}-P_{i j}^{*}\right)}{P_{i j}^{*}\left(1-P_{i j}^{*}\right)}+\left(\frac{\partial \delta_{i}}{\partial \boldsymbol{\zeta}_{i}}\right)^{t} \frac{\left(c_{i j}-\delta_{i}\right)}{\delta_{i}\left(1-\delta_{i}\right)}+\right. \\
& \left.\left(\frac{\partial P_{i j}}{\partial \boldsymbol{\zeta}_{i}}\right)^{t} \frac{\left(1-c_{i j}\right)\left(x_{i j}-P_{i j}\right)}{P_{i j}\left(1-P_{i j}\right)}\right] P\left(\boldsymbol{x}_{\cdot j}, \boldsymbol{c}_{\cdot j} \mid \theta, \boldsymbol{\zeta}\right)+ \\
& \frac{\partial}{\partial \boldsymbol{\zeta}_{i}} P\left(\boldsymbol{x}_{\cdot j}, \boldsymbol{c}_{\cdot j} \mid \theta, \boldsymbol{\zeta}\right)\left[\left(\frac{\partial P_{i j}^{*}}{\partial \boldsymbol{\zeta}_{i}}\right)^{t} \frac{c_{i j}\left(x_{i j}-P_{i j}^{*}\right)}{P_{i j}^{*}\left(1-P_{i j}^{*}\right)}+\right. \\
& \left.\left(\frac{\partial \delta_{i}}{\partial \boldsymbol{\zeta}_{i}}\right)^{t} \frac{\left(c_{i j}-\delta_{i}\right)}{\delta_{i}\left(1-\delta_{i}\right)}+\left(\frac{\partial P_{i j}}{\partial \boldsymbol{\zeta}_{i}}\right)^{t} \frac{\left(1-c_{i j}\right)\left(x_{i j}-P_{i j}\right)}{P_{i j}\left(1-P_{i j}\right)}\right] \\
& =\left\{\frac{\partial^{2} P_{i j}^{*}}{\partial \boldsymbol{\zeta}_{i} \partial \boldsymbol{\zeta}_{i}^{t}} \frac{c_{i j}\left(x_{i j}-P_{i j}^{*}\right)}{P_{i j}^{*}\left(1-P_{i j}^{*}\right)}+\frac{\partial}{\partial \boldsymbol{\zeta}_{i}}\left[\frac{c_{i j}\left(x_{i j}-P_{i j}^{*}\right)}{P_{i j}^{*}\left(1-P_{i j}^{*}\right)}\right]\left(\frac{\partial P_{i j}^{*}}{\partial \boldsymbol{\zeta}_{i}}\right)^{t}+\right. \\
& \frac{\partial^{2} \delta_{i}}{\partial \boldsymbol{\zeta}_{i} \partial \boldsymbol{\zeta}_{i}^{t}} \frac{\left(c_{i j}-\delta_{i}\right)}{\delta_{i}\left(1-\delta_{i}\right)}+\frac{\partial}{\partial \boldsymbol{\zeta}_{i}}\left[\frac{\left(c_{i j}-\delta_{i}\right)}{\delta_{i}\left(1-\delta_{i}\right)}\right]\left(\frac{\partial \delta_{i}}{\partial \boldsymbol{\zeta}_{i}}\right)^{t}+ \\
& \left.\frac{\partial^{2} P_{i j}}{\partial \boldsymbol{\zeta}_{i} \partial \boldsymbol{\zeta}_{i}^{t}} \frac{\left(1-c_{i j}\right)\left(x_{i j}-P_{i j}\right)}{P_{i j}\left(1-P_{i j}\right)}+\frac{\partial}{\partial \boldsymbol{\zeta}_{i}}\left[\frac{\left(1-c_{i j}\right)\left(x_{i j}-P_{i j}\right)}{P_{i j}\left(1-P_{i j}\right)}\right]\left(\frac{\partial P_{i j}}{\partial \boldsymbol{\zeta}_{i}}\right)^{t}\right\} P\left(\boldsymbol{x}_{\cdot j}, \boldsymbol{c}_{\cdot j} \mid \theta, \boldsymbol{\zeta}\right)+ \\
& {\left[\frac{\partial P_{i j}^{*}}{\partial \boldsymbol{\zeta}_{i}} \frac{c_{i j}\left(x_{i j}-P_{i j}^{*}\right)}{P_{i j}^{*}\left(1-P_{i j}^{*}\right)}+\frac{\partial \delta_{i}}{\partial \boldsymbol{\zeta}_{i}} \frac{\left(c_{i j}-\delta_{i}\right)}{\delta_{i}\left(1-\delta_{i}\right)}+\frac{\partial P_{i j}}{\partial \boldsymbol{\zeta}_{i}} \frac{\left(1-c_{i j}\right)\left(x_{i j}-P_{i j}\right)}{P_{i j}\left(1-P_{i j}\right)}\right]}
\end{aligned}
$$




$$
\begin{aligned}
& {\left[\left(\frac{\partial P_{i j}^{*}}{\partial \boldsymbol{\zeta}_{i}}\right)^{t} \frac{c_{i j}\left(x_{i j}-P_{i j}^{*}\right)}{P_{i j}^{*}\left(1-P_{i j}^{*}\right)}+\left(\frac{\partial \delta_{i}}{\partial \boldsymbol{\zeta}_{i}}\right)^{t} \frac{\left(c_{i j}-\delta_{i}\right)}{\delta_{i}\left(1-\delta_{i}\right)}+\left(\frac{\partial P_{i j}}{\partial \boldsymbol{\zeta}_{i}}\right)^{t} \frac{\left(1-c_{i j}\right)\left(x_{i j}-P_{i j}\right)}{P_{i j}\left(1-P_{i j}\right)}\right] P\left(\boldsymbol{x} \cdot j, \boldsymbol{c}_{\cdot j} \mid \theta, \boldsymbol{\zeta}\right)} \\
& =\left\{\frac{\partial^{2} P_{i j}^{*}}{\partial \boldsymbol{\zeta}_{i} \partial \boldsymbol{\zeta}_{i}^{t}} \frac{c_{i j}\left(x_{i j}-P_{i j}^{*}\right)}{P_{i j}^{*}\left(1-P_{i j}^{*}\right)}-\left(\frac{\partial P_{i j}^{*}}{\partial \boldsymbol{\zeta}_{i}}\right)\left(\frac{\partial P_{i j}^{*}}{\partial \boldsymbol{\zeta}_{i}}\right)^{t} c_{i j}\left[\frac{\left(x_{i j}-P_{i j}^{*}\right)}{P_{i j}^{*}\left(1-P_{i j}^{*}\right)}\right]^{2}+\right. \\
& \frac{\partial^{2} \delta_{i}}{\partial \boldsymbol{\zeta}_{i} \partial \boldsymbol{\zeta}_{i}^{t}} \frac{\left(c_{i j}-\delta_{i}\right)}{\delta_{i}\left(1-\delta_{i}\right)}-\left(\frac{\partial \delta_{i}}{\partial \boldsymbol{\zeta}_{i}}\right)\left(\frac{\partial \delta_{i}}{\partial \boldsymbol{\zeta}_{i}}\right)^{t}\left[\frac{\left(c_{i j}-\delta_{i}\right)}{\delta_{i}\left(1-\delta_{i}\right)}\right]^{2}+ \\
& \frac{\partial^{2} P_{i j}}{\partial \boldsymbol{\zeta}_{i} \partial \boldsymbol{\zeta}_{i}^{t}} \frac{\left(1-c_{i j}\right)\left(x_{i j}-P_{i j}\right)}{P_{i j}\left(1-P_{i j}\right)}-\left(\frac{\partial P_{i j}}{\partial \boldsymbol{\zeta}_{i}}\right)\left(\frac{\partial P_{i j}}{\partial \boldsymbol{\zeta}_{i}}\right)^{t}\left(1-c_{i j}\right)\left[\frac{\left(x_{i j}-P_{i j}\right)}{P_{i j}\left(1-P_{i j}\right)}\right]^{2}+ \\
& \left(\frac{\partial P_{i j}^{*}}{\partial \boldsymbol{\zeta}_{i}}\right)\left(\frac{\partial P_{i j}^{*}}{\partial \boldsymbol{\zeta}_{i}}\right)^{t}\left[\frac{c_{i j}\left(x_{i j}-P_{i j}^{*}\right)}{P_{i j}^{*}\left(1-P_{i j}^{*}\right)}\right]^{2}+\left(\frac{\partial P_{i j}^{*}}{\partial \boldsymbol{\zeta}_{i}}\right)\left(\frac{\partial \delta_{i}}{\partial \boldsymbol{\zeta}_{i}}\right)^{t} \frac{c_{i j}\left(x_{i j}-P_{i j}^{*}\right)}{P_{i j}^{*}\left(1-P_{i j}^{*}\right)} \frac{\left(c_{i j}-\delta_{i}\right)}{\delta_{i}\left(1-\delta_{i}\right)}+ \\
& \left(\frac{\partial P_{i j}^{*}}{\partial \boldsymbol{\zeta}_{i}}\right)\left(\frac{\partial P_{i j}}{\partial \boldsymbol{\zeta}_{i}}\right)^{t} \frac{c_{i j}\left(x_{i j}-P_{i j}^{*}\right)}{P_{i j}^{*}\left(1-P_{i j}^{*}\right)} \frac{\left(1-c_{i j}\right)\left(x_{i j}-P_{i j}\right)}{P_{i j}\left(1-P_{i j}\right)}+ \\
& \left(\frac{\partial \delta_{i}}{\partial \boldsymbol{\zeta}_{i}}\right)\left(\frac{\partial P_{i j}^{*}}{\partial \boldsymbol{\zeta}_{i}}\right)^{t} \frac{c_{i j}\left(x_{i j}-P_{i j}^{*}\right)}{P_{i j}^{*}\left(1-P_{i j}^{*}\right)} \frac{\left(c_{i j}-\delta_{i}\right)}{\delta_{i}\left(1-\delta_{i}\right)}+ \\
& \left(\frac{\partial \delta_{i}}{\partial \boldsymbol{\zeta}_{i}}\right)\left(\frac{\partial \delta_{i}}{\partial \boldsymbol{\zeta}_{i}}\right)^{t}\left(\frac{\left(c_{i j}-\delta_{i}\right)}{\delta_{i}\left(1-\delta_{i}\right)}\right)^{2}+\left(\frac{\partial \delta_{i}}{\partial \boldsymbol{\zeta}_{i}}\right)\left(\frac{\partial P_{i j}}{\partial \boldsymbol{\zeta}_{i}}\right)^{t} \frac{\left(1-c_{i j}\right)\left(x_{i j}-P_{i j}\right)}{P_{i j}\left(1-P_{i j}\right)} \frac{\left(c_{i j}-\delta_{i}\right)}{\delta_{i}\left(1-\delta_{i}\right)}+ \\
& \left(\frac{\partial P_{i j}}{\partial \boldsymbol{\zeta}_{i}}\right)\left(\frac{\partial P_{i j}^{*}}{\partial \boldsymbol{\zeta}_{i}}\right)^{t} \frac{\left(1-c_{i j}\right)\left(x_{i j}-P_{i j}\right)}{P_{i j}\left(1-P_{i j}\right)} \frac{c_{i j}\left(x_{i j}-P_{i j}^{*}\right)}{P_{i j}^{*}\left(1-P_{i j}^{*}\right)}+ \\
& \left(\frac{\partial P_{i j}}{\partial \boldsymbol{\zeta}_{i}}\right)\left(\frac{\partial \delta_{i}}{\partial \boldsymbol{\zeta}_{i}}\right)^{t} \frac{\left(1-c_{i j}\right)\left(x_{i j}-P_{i j}\right)}{P_{i j}\left(1-P_{i j}\right)} \frac{\left(c_{i j}-\delta_{i}\right)}{\delta_{i}\left(1-\delta_{i}\right)}+ \\
& \left.\left(\frac{\partial P_{i j}}{\partial \boldsymbol{\zeta}_{i}}\right)\left(\frac{\partial P_{i j}}{\partial \boldsymbol{\zeta}_{i}}\right)^{t}\left(\frac{\left(1-c_{i j}\right)\left(x_{i j}-P_{i j}\right)}{P_{i j}\left(1-P_{i j}\right)}\right)^{2}\right\} P\left(\boldsymbol{x}_{\cdot j}, \boldsymbol{c}_{\cdot j} \mid \theta, \boldsymbol{\zeta}\right) \\
& =\left\{\frac{\partial^{2} P_{i j}^{*}}{\partial \boldsymbol{\zeta}_{i} \partial \boldsymbol{\zeta}_{i}^{t}} \frac{c_{i j}\left(x_{i j}-P_{i j}^{*}\right)}{P_{i j}^{*}\left(1-P_{i j}^{*}\right)}+\frac{\partial^{2} P_{i j}}{\partial \boldsymbol{\zeta}_{i} \partial \boldsymbol{\zeta}_{i}^{t}} \frac{\left(1-c_{i j}\right)\left(x_{i j}-P_{i j}\right)}{P_{i j}\left(1-P_{i j}\right)}-\right. \\
& \left(\frac{\partial P_{i j}^{*}}{\partial \boldsymbol{\zeta}_{i}}\right)\left(\frac{\partial P_{i j}^{*}}{\partial \boldsymbol{\zeta}_{i}}\right)^{t} c_{i j}\left(1-c_{i j}\right)\left[\frac{\left(x_{i j}-P_{i j}^{*}\right)}{P_{i j}^{*}\left(1-P_{i j}^{*}\right)}\right]^{2}- \\
& \left(\frac{\partial P_{i j}}{\partial \boldsymbol{\zeta}_{i}}\right)\left(\frac{\partial P_{i j}}{\partial \boldsymbol{\zeta}_{i}}\right)^{t} c_{i j}\left(1-c_{i j}\right)\left(\frac{\left(x_{i j}-P_{i j}\right)}{P_{i j}\left(1-P_{i j}\right)}\right)^{2}+ \\
& {\left[\left(\frac{\partial P_{i j}^{*}}{\partial \boldsymbol{\zeta}_{i}}\right)\left(\frac{\partial \delta_{i}}{\partial \boldsymbol{\zeta}_{i}}\right)^{t}+\left(\frac{\partial \delta_{i}}{\partial \boldsymbol{\zeta}_{i}}\right)\left(\frac{\partial P_{i j}^{*}}{\partial \boldsymbol{\zeta}_{i}}\right)^{t}\right] \frac{c_{i j}\left(x_{i j}-P_{i j}^{*}\right)}{P_{i j}^{*}\left(1-P_{i j}^{*}\right)} \frac{\left(c_{i j}-\delta_{i}\right)}{\delta_{i}\left(1-\delta_{i}\right)}+} \\
& {\left[\left(\frac{\partial P_{i j}^{*}}{\partial \boldsymbol{\zeta}_{i}}\right)\left(\frac{\partial P_{i j}}{\partial \boldsymbol{\zeta}_{i}}\right)^{t}+\left(\frac{\partial P_{i j}}{\partial \boldsymbol{\zeta}_{i}}\right)\left(\frac{\partial P_{i j}^{*}}{\partial \boldsymbol{\zeta}_{i}}\right)^{t}\right] \frac{\left(1-c_{i j}\right)\left(x_{i j}-P_{i j}\right)}{P_{i j}\left(1-P_{i j}\right)} \frac{c_{i j}\left(x_{i j}-P_{i j}^{*}\right)}{P_{i j}^{*}\left(1-P_{i j}^{*}\right)}+}
\end{aligned}
$$




$$
\left.\left[\left(\frac{\partial P_{i j}}{\partial \boldsymbol{\zeta}_{i}}\right)\left(\frac{\partial \delta_{i}}{\partial \boldsymbol{\zeta}_{i}}\right)^{t}+\left(\frac{\partial \delta_{i}}{\partial \boldsymbol{\zeta}_{i}}\right)\left(\frac{\partial P_{i j}}{\partial \boldsymbol{\zeta}_{i}}\right)^{t}\right] \frac{\left(1-c_{i j}\right)\left(x_{i j}-P_{i j}\right)}{P_{i j}\left(1-P_{i j}\right)} \frac{\left(c_{i j}-\delta_{i}\right)}{\delta_{i}\left(1-\delta_{i}\right)}\right\} P\left(\boldsymbol{x}_{\cdot j}, \boldsymbol{c}_{\cdot j} \mid \theta, \boldsymbol{\zeta}\right)
$$

A penúltima igualdade segue dos resultados obtidos em A.17)-A.19.

$$
\begin{aligned}
& \frac{\partial^{2} P_{i j}^{*}}{\partial a_{i}^{2}}=\frac{\theta_{j}-b_{2 i}}{\gamma_{i}} \frac{\partial P_{i j}^{*}}{\partial a_{i}}\left(\gamma_{i}-2 P_{i j}^{*}\right) \\
& =\frac{\left(\theta_{j}-b_{2 i}\right)^{2}}{\gamma_{i}^{2}} P_{i j}^{*}\left(\gamma_{i}-P_{i j}^{*}\right)\left(\gamma_{i}-2 P_{i j}^{*}\right) \\
& \frac{\partial^{2} P_{i j}^{*}}{\partial a_{i} \partial b_{1 i}}=\frac{\theta_{j}-b_{2 i}}{\gamma_{i}} \frac{\partial P_{i j}^{*}}{\partial b_{1 i}}\left(\gamma_{i}-2 P_{i j}^{*}\right)=0 \\
& \frac{\partial^{2} P_{i j}^{*}}{\partial a_{i} \partial b_{2 i}}=\left(\frac{\theta_{j}}{\gamma_{i}} \frac{\partial P_{i j}^{*}}{\partial b_{2 i}}-\frac{P_{i j}^{*}}{\gamma_{i}}-\frac{b_{2 i}}{\gamma_{i}} \frac{\partial P_{i j}^{*}}{\partial b_{2 i}}\right)\left(\gamma_{i}-P_{i j}^{*}\right)-\left(\frac{\theta_{j}}{\gamma_{i}} P_{i j}^{*}-\frac{b_{2 i}}{\gamma_{i}} P_{i j}^{*}\right) \frac{\partial P_{i j}^{*}}{\partial b_{2 i}} \\
& =\frac{\partial P_{i j}^{*}}{\partial b_{2 i}}\left[\left(\frac{\theta_{j}}{\gamma_{i}}-\frac{b_{2 i}}{\gamma_{i}}\right)\left(\gamma_{i}-P_{i j}^{*}\right)-P_{i j}^{*}\left(\frac{\theta_{j}}{\gamma_{i}}-\frac{b_{2 i}}{\gamma_{i}}\right)\right]-\frac{P_{i j}^{*}}{\gamma_{i}}\left(\gamma_{i}-P_{i j}^{*}\right) \\
& =\frac{\partial P_{i j}^{*}}{\partial b_{2 i}}\left(\frac{\theta_{j}}{\gamma_{i}}-\frac{b_{2 i}}{\gamma_{i}}\right)\left(\gamma_{i}-2 P_{i j}^{*}\right)-\frac{P_{i j}^{*}}{\gamma_{i}}\left(\gamma_{i}-P_{i j}^{*}\right) \\
& =\frac{-a_{i}}{\gamma_{i}} P_{i j}\left(\gamma_{i}-P_{i j}^{*}\right)\left(\frac{\theta_{j}}{\gamma_{i}}-\frac{b_{2 i}}{\gamma_{i}}\right)\left(\gamma_{i}-2 P_{i j}^{*}\right)-\frac{P_{i j}^{*}}{\gamma_{i}}\left(\gamma_{i}-P_{i j}^{*}\right) \\
& =\frac{P_{i j}^{*}\left(\gamma_{i}-P_{i j}^{*}\right)}{\gamma_{i}}\left[\frac{-a_{i}}{\gamma_{i}}\left(\theta_{j}-b_{2 i}\right)\left(\gamma_{i}-2 P_{i j}^{*}\right)-1\right] \\
& \frac{\partial^{2} P_{i j}^{*}}{\partial a_{i} \partial \gamma_{i}}=\frac{1}{\gamma_{i}} \frac{\partial P_{i j}^{*}}{\partial a_{i}}=\frac{\left(\theta_{j}-b_{2 i}\right)}{\gamma_{i}^{2}} P_{i j}^{*}\left(1-P_{i j}^{*}\right) \\
& \frac{\partial^{2} P_{i j}^{*}}{\partial a_{i} \partial \delta_{i}}=\frac{\partial^{2} P_{i j}^{*}}{\partial b_{1 i}^{2}}=\frac{\partial^{2} P_{i j}^{*}}{\partial b_{1 i} \partial b_{2 i}}=\frac{\partial^{2} P_{i j}^{*}}{\partial b_{1 i} \partial \gamma_{i}}=\frac{\partial^{2} P_{i j}^{*}}{\partial b_{1 i} \partial \delta_{i}}=\frac{\partial^{2} P_{i j}^{*}}{\partial b_{2 i} \partial \delta_{i}}=\frac{\partial^{2} P_{i j}^{*}}{\partial \gamma_{i} \partial \delta_{i}}=0 \\
& \frac{\partial^{2} P_{i j}^{*}}{\partial b_{2 i}^{2}}=\frac{-a_{i}}{\gamma_{i}} \frac{\partial P_{i j}^{*}}{\partial b_{2 i}}\left(\gamma_{i}-P_{i j}^{*}\right)+\frac{a_{i}}{\gamma_{i}} P_{i j}^{*} \frac{\partial P_{i j}^{*}}{\partial b_{2 i}} \\
& =\frac{-a_{i}}{\gamma_{i}}\left(\frac{-a_{i}}{\gamma_{i}}\right) P_{i j}^{*}\left(\gamma_{i}-P_{i j}^{*}\right)\left(\gamma_{i}-2 P_{i j}^{*}\right) \\
& =\frac{a_{i}^{2}}{\gamma_{i}^{2}} P_{i j}^{*}\left(\gamma_{i}-P_{i j}^{*}\right)\left(\gamma_{i}-2 P_{i j}^{*}\right)
\end{aligned}
$$




$$
\begin{aligned}
& \frac{\partial^{2} P_{i j}^{*}}{\partial b_{2 i} \partial \gamma_{i}}=\frac{1}{\gamma_{i}} \frac{\partial P_{i j}^{*}}{\partial b_{2 i}}=\frac{-a_{i}}{\gamma_{i}^{2}} P_{i j}^{*}\left(\gamma_{i}-P_{i j}^{*}\right) \\
& \frac{\partial^{2} P_{i j}^{*}}{\partial \gamma_{i}^{2}}=\frac{P_{i j}^{*}-P_{i j}^{*}}{\gamma_{i}^{2}}=0 \\
& \frac{\partial^{2} \delta_{i}}{\partial a_{i}^{2}}=\frac{\partial^{2} \delta_{i}}{\partial a_{i} \partial b_{1 i}}=\frac{\partial^{2} \delta_{i}}{\partial a_{i} \partial b_{2 i}}=\frac{\partial^{2} \delta_{i}}{\partial a_{i} \partial \gamma_{i}}=\frac{\partial^{2} \delta_{i}}{\partial a_{i} \partial \delta_{i}}=\frac{\partial^{2} \delta_{i}}{\partial b_{1 i}^{2}}=\frac{\partial^{2} \delta_{i}}{\partial b_{1 i} \partial b_{2 i}}= \\
& \frac{\partial^{2} \delta_{i}}{\partial b_{1 i} \partial \gamma_{i}}=\frac{\partial^{2} \delta_{i}}{\partial b_{1 i} \partial \delta_{i}}=\frac{\partial^{2} \delta_{i}}{\partial b_{2 i}^{2}}=\frac{\partial^{2} \delta_{i}}{\partial b_{2 i} \partial \gamma_{i}}=\frac{\partial^{2} \delta_{i}}{\partial b_{2 i} \partial \delta_{i}}=\frac{\partial^{2} \delta_{i}}{\partial \gamma_{i}^{2}}=\frac{\partial^{2} \delta_{i}}{\partial \gamma_{i} \partial \delta_{i}}=\frac{\partial^{2} \delta_{i}}{\partial \delta_{i}^{2}}=0 \\
& \frac{\partial^{2} P_{i j}}{\partial a_{i}^{2}}=\left(\theta_{j}-b_{1 i}\right) \frac{\partial P_{i j}}{\partial a_{i}}\left(1-P_{i j}\right)-\left(\theta_{j}-b_{1 i}\right) P_{i j} \frac{\partial P_{i j}}{\partial a_{i}} \\
& =\left(\theta_{j}-b_{1 i}\right)^{2} P_{i j}\left(1-P_{i j}\right)\left(1-2 P_{i j}\right) \\
& \frac{\partial^{2} P_{i j}}{\partial a_{i} \partial b_{1 i}}=-P_{i j}-a_{i} \frac{\partial P_{i j}}{\partial a_{i}}+P_{i j}^{2}+a_{i} 2 P_{i j} \frac{\partial P_{i j}}{\partial a_{i}} \\
& =-P_{i j}\left(1-P_{i j}\right)-a_{i} \frac{\partial P_{i j}}{\partial a_{i}}\left(1-2 P_{i j}\right) \\
& =-P_{i j}\left(1-P_{i j}\right)-a_{i}\left(\theta_{j}-b_{1 i}\right) P_{i j}\left(1-P_{i j}\right)\left(1-2 P_{i j}\right) \\
& =-P_{i j}\left(1-P_{i j}\right)\left[1+a_{i}\left(\theta_{j}-b_{1 i}\right)\left(1-2 P_{i j}\right)\right] \\
& \frac{\partial^{2} P_{i j}}{\partial a_{i} \partial b_{2 i}}=\frac{\partial^{2} P_{i j}}{\partial a_{i} \partial \gamma_{i}}=\frac{\partial^{2} P_{i j}}{\partial a_{i} \partial \delta_{i}}=\frac{\partial^{2} P_{i j}}{\partial b_{1 i} \partial b_{2 i}}=\frac{\partial^{2} P_{i j}}{\partial b_{1 i} \partial \gamma_{i}}=\frac{\partial^{2} P_{i j}}{\partial b_{1 i} \partial \delta_{i}}= \\
& \frac{\partial^{2} P_{i j}}{\partial b_{2 i}^{2}}=\frac{\partial^{2} P_{i j}}{\partial b_{2 i} \partial \gamma_{i}}=\frac{\partial^{2} P_{i j}}{\partial b_{2 i} \partial \delta_{i}}=\frac{\partial^{2} P_{i j}}{\partial \gamma_{i}^{2}}=\frac{\partial^{2} P_{i j}}{\partial \gamma_{i} \partial \delta_{i}}=\frac{\partial^{2} P_{i j}}{\partial \delta_{i}^{2}}=0 \\
& \frac{\partial^{2} P_{i j}}{\partial b_{1 i}^{2}}=-a_{i} \frac{\partial P_{i j}}{\partial b_{1 i}}\left(1-P_{i j}\right)+a_{i} P_{i j} \frac{\partial P_{i j}}{\partial b_{1 i}} \\
& =-a_{i}\left(1-2 P_{i j}\right)\left(-a_{i}\right) P_{i j}\left(1-P_{i j}\right) \\
& =a_{i}^{2} P_{i j}\left(1-P_{i j}\right)\left(1-2 P_{i j}\right)
\end{aligned}
$$




$$
\begin{aligned}
E\left[c_{l j}\left(x_{l j}-P_{l}^{*}\right) c_{i j}\left(x_{i j}-P_{i}^{*}\right) \mid \theta\right] & =E\left[c_{l j}\left(x_{l j}-P_{l}^{*}\right) \mid \theta\right] E\left[c_{i j}\left(x_{i j}-P_{i}^{*}\right) \mid \theta\right] \\
& =\left(E\left[c_{l j} x_{l j}\right]-E\left[c_{l j}\right] P_{l}^{*}\right)\left(E\left[c_{i j} x_{i j}\right]-E\left[c_{i j}\right] P_{i}^{*}\right) \\
& =\left(\delta_{l} P_{l}^{*}-\delta_{l} P_{l}^{*}\right)\left(\delta_{i} P_{i}^{*}-\delta_{i} P_{i}^{*}\right)=0
\end{aligned}
$$

Analogamente, mostra-se que

$$
\begin{aligned}
& E\left[c_{l j}\left(x_{l j}-P_{l}^{*}\right)\left(c_{i j}-\delta_{i}\right) \mid \theta\right]=E\left[c_{l j}\left(x_{l j}-P_{l}^{*}\right)\left(1-c_{i j}\right)\left(x_{i j}-P_{i}\right) \mid \theta\right]= \\
& \quad E\left[\left(c_{l j}-\delta_{l}\right) c_{i j}\left(x_{i j}-P_{i}^{*}\right) \mid \theta\right]=E\left[\left(c_{l j}-\delta_{l}\right)\left(c_{i j}-\delta_{i}\right) \mid \theta\right]= \\
& E\left[\left(c_{l j}-\delta_{l}\right)\left(1-c_{i j}\right)\left(x_{i j}-P_{i}\right) \mid \theta\right]=E\left[\left(1-c_{l j}\right)\left(x_{l j}-P_{l}\right) c_{i j}\left(x_{i j}-P_{i}^{*}\right) \mid \theta\right]= \\
& E\left[\left(1-c_{l j}\right)\left(x_{l j}-P_{l}\right)\left(c_{i j}-\delta_{i}\right) \mid \theta\right]=E\left[\left(1-c_{l j}\right)\left(x_{l j}-P_{l}\right)\left(1-c_{i j}\right)\left(x_{i j}-P_{i}\right) \mid \theta\right]= \\
& E\left[c_{i j}\left(x_{i j}-P_{i}^{*}\right) \mid \theta\right]=E\left[\left(1-c_{i j}\right)\left(x_{i j}-P_{i}\right) \mid \theta\right]=0 \\
& \\
& \begin{aligned}
E\left[c_{i j}\left(x_{i j}-P_{i}^{*}\right)\left(c_{i j}-\delta_{i}\right) \mid \theta\right] & =E\left[c_{i j}^{2} x_{i j}-c_{i j} x_{i j} \delta_{i}-c_{i j}^{2} P_{i}^{*}+c_{i j} \delta_{i} P_{i}^{*} \mid \theta\right] \\
& =\delta_{i} P_{i}^{*}-\delta_{i}^{2} P_{i}^{*}-\left[\delta_{i}\left(1-\delta_{i}\right)+\delta_{i}^{2}\right] P_{i}^{*}+\delta_{i}^{2} P_{i}^{*}=0
\end{aligned}
\end{aligned}
$$

Analogamente, mostra-se que

$$
\begin{aligned}
& E {\left[c_{i j}\left(x_{i j}-P_{i}^{*}\right)\left(1-c_{i j}\right)\left(x_{i j}-P_{i}\right) \mid \theta\right]=E\left[\left(c_{i j}-\delta_{i}\right)\left(1-c_{i j}\right)\left(x_{i j}-P_{i}\right) \mid \theta\right]=0 \quad \text { (A.37) } } \\
& \frac{\partial E[l(\boldsymbol{\zeta})]}{\partial \boldsymbol{\zeta}_{i}}=\sum_{k=1}^{q}\left\{\bar{r}_{2 i k} \frac{1}{\delta_{i} P_{i k}^{*}}\left[\frac{\partial \delta_{i}}{\partial \boldsymbol{\zeta}_{i}} P_{i k}^{*}+\delta_{i} \frac{\partial P_{i k}^{*}}{\partial \boldsymbol{\zeta}_{i}}\right]+\right. \\
&\left(\bar{f}_{2 i k}-\bar{r}_{2 i k}\right) \frac{1}{\delta_{i}\left(1-P_{i k}^{*}\right)}\left[\frac{\partial \delta_{i}}{\partial \boldsymbol{\zeta}_{i}}\left(1-P_{i k}^{*}\right)-\delta_{i} \frac{\partial P_{i k}^{*}}{\partial \boldsymbol{\zeta}_{i}}\right]+ \\
&\left(\bar{r}_{1 i k} \frac{1}{\left(1-\delta_{i}\right) P_{i k}}\left[-\frac{\partial \delta_{i}}{\partial \boldsymbol{\zeta}_{i}} P_{i k}+\left(1-\delta_{i}\right) \frac{\partial P_{i k}}{\partial \boldsymbol{\zeta}_{i}}\right]+\right. \\
&\left.\left(\bar{f}_{1 i k}-\bar{r}_{1 i k}\right) \frac{1}{\left(1-\delta_{i}\right)\left(1-P_{i k}\right)}\left[-\frac{\partial \delta_{i}}{\partial \boldsymbol{\zeta}_{i}}\left(1-P_{i k}\right)+\left(1-\delta_{i}\right) \frac{\partial P_{i k}}{\partial \boldsymbol{\zeta}_{i}}\right]\right\} \\
&=\sum_{k=1}^{q}\left\{\frac { 1 } { \delta _ { i } P _ { i k } ^ { * } ( 1 - P _ { i k } ^ { * } ) } \left[\bar{r}_{2 i k} P_{i k}^{*}\left(\partial \delta_{i} / \partial \boldsymbol{\zeta}_{i}\right)+\bar{r}_{2 i k} \delta_{i}\left(\partial P_{i k}^{*} / \partial \boldsymbol{\zeta}_{i}\right)-\bar{r}_{2 i k}\left(P_{i k}^{*}\right)^{2}\left(\partial \delta_{i} / \partial \boldsymbol{\zeta}_{i}\right)-\right.\right. \\
& \bar{r}_{2 i k} P_{i k}^{*} \delta_{i}\left(\partial P_{i k}^{*} / \partial \boldsymbol{\zeta}_{i}\right)+\bar{f}_{2 i k} P_{i k}^{*}\left(\partial \delta_{i} / \partial \boldsymbol{\zeta}_{i}\right)-\bar{f}_{2 i k}\left(P_{i k}^{*}\right)^{2}\left(\partial \delta_{i} / \partial \boldsymbol{\zeta}_{i}\right)-\bar{f}_{2 i k} P_{i k}^{*} \delta_{i}\left(\partial P_{i k}^{*} / \partial \boldsymbol{\zeta}_{i}\right)+ \\
&\left.\bar{r}_{2 i k} P_{i k}^{*}\left(\partial \delta_{i} / \partial \boldsymbol{\zeta}_{i}\right)+\bar{r}_{2 i k}\left(P_{i k}^{*}\right)^{2}\left(\partial \delta_{i} / \partial \boldsymbol{\zeta}_{i}\right)+\bar{r}_{2 i k} P_{i k}^{*} \delta_{i}\left(\partial P_{i k}^{*} / \partial \boldsymbol{\zeta}_{i}\right)\right]+
\end{aligned}
$$




$$
\begin{aligned}
& \frac{1}{\left(1-\delta_{i}\right) P_{i k}\left(1-P_{i k}\right)}\left[\bar{r}_{1 i k} P_{i k}\left(-\partial \delta_{i} / \partial \boldsymbol{\zeta}_{i}\right)+\bar{r}_{1 i k} P_{i k}^{2}\left(\partial \delta_{i} / \partial \boldsymbol{\zeta}_{i}\right)+\bar{r}_{1 i k}\left(\partial P_{i k} / \partial \boldsymbol{\zeta}_{i}\right)-\right. \\
& \bar{r}_{1 i k} \delta_{i}\left(\partial P_{i k} / \partial \boldsymbol{\zeta}_{i}\right)-\bar{r}_{1 i k} P_{i k}\left(\partial P_{i k} / \partial \boldsymbol{\zeta}_{i}\right)+\bar{r}_{1 i k} P_{i k} \delta_{i}\left(\partial P_{i k} / \partial \boldsymbol{\zeta}_{i}\right)+\bar{f}_{1 i k} P_{i k}\left(-\partial \delta_{i} / \partial \boldsymbol{\zeta}_{i}\right)+ \\
& \bar{f}_{1 i k} P_{i k}^{2}\left(\partial \delta_{i} / \partial \boldsymbol{\zeta}_{i}\right)+\bar{r}_{1 i k} P_{i k}\left(\partial P_{i k} / \partial \boldsymbol{\zeta}_{i}\right)-\bar{r}_{1 i k} P_{i k} \delta_{i}\left(\partial P_{i k} / \partial \boldsymbol{\zeta}_{i}\right)+\bar{r}_{1 i k} P_{i k}\left(\partial \delta_{i} / \partial \boldsymbol{\zeta}_{i}\right)- \\
& \left.\left.\bar{r}_{1 i k} P_{i k}^{2}\left(\partial \delta_{i} / \partial \boldsymbol{\zeta}_{i}\right)-\bar{f}_{1 i k} P_{i k}\left(\partial P_{i k} / \partial \boldsymbol{\zeta}_{i}\right)+\bar{f}_{1 i k} P_{i k} \delta_{i}\left(\partial P_{i k} / \partial \boldsymbol{\zeta}_{i}\right)\right]\right\} \\
& =\sum_{k=1}^{q}\left\{\frac{\left(\bar{r}_{2 i k}-\bar{f}_{2 i k} P_{i k}^{*}\right) \delta_{i}\left(\partial P_{i k}^{*} / \partial \boldsymbol{\zeta}_{i}\right)+\bar{f}_{2 i k} P_{i k}^{*}\left(1-P_{i k}^{*}\right)\left(\partial \delta_{i} / \partial \boldsymbol{\zeta}_{i}\right)}{\delta_{i} P_{i k}^{*}\left(1-P_{i k}^{*}\right)}+\right. \\
& \left.\frac{\left(\bar{r}_{1 i k}-\bar{f}_{1 i k} P_{i k}\right)\left(1-\delta_{i}\right)\left(\partial P_{i k} / \partial \boldsymbol{\zeta}_{i}\right)-\bar{f}_{1 i k} P_{i k}\left(1-P_{i k}\right)\left(\partial \delta_{i} / \partial \boldsymbol{\zeta}_{i}\right)}{\left(1-\delta_{i}\right) P_{i k}\left(1-P_{i k}\right)}\right\} \\
& =\sum_{k=1}^{q}\left\{\frac{\left(\bar{r}_{2 i k}-\bar{f}_{2 i k} P_{i k}^{*}\right)}{P_{i k}^{*}\left(1-P_{i k}^{*}\right)}\left(\frac{\partial P_{i k}^{*}}{\partial \boldsymbol{\zeta}_{i}}\right)+\frac{\bar{f}_{2 i k}}{\delta_{i}}\left(\frac{\partial \delta_{i}}{\partial \boldsymbol{\zeta}_{i}}\right)+\right. \\
& \left.\frac{\left(\bar{r}_{1 i k}-\bar{f}_{1 i k} P_{i k}\right)}{P_{i k}\left(1-P_{i k}\right)}\left(\frac{\partial P_{i k}}{\partial \zeta_{i}}\right)-\frac{\bar{f}_{1 i k}}{\left(1-\delta_{i}\right)}\left(\frac{\partial \delta_{i}}{\partial \zeta_{i}}\right)\right\} \\
& =\frac{\partial}{\boldsymbol{\zeta}_{i}}\left\{\sum _ { k = 1 } ^ { q } \left\{\frac{\left(\bar{r}_{2 i k}-\bar{f}_{2 i k} P_{i k}^{*}\right)}{P_{i k}^{*}\left(1-P_{i k}^{*}\right)}\left(\frac{\partial P_{i k}^{*}}{\partial \boldsymbol{\zeta}_{i}}\right)+\right.\right. \\
& \left.\frac{\left(\bar{r}_{1 i k}-\bar{f}_{1 i k} P_{i k}\right)}{P_{i k}\left(1-P_{i k}\right)}\left(\frac{\partial P_{i k}}{\partial \boldsymbol{\zeta}_{i}}\right)-\frac{\bar{f}_{2 i k}-\left(\bar{f}_{2 i k}+\bar{f}_{1 i k}\right) \delta_{i}}{\delta_{i}\left(1-\delta_{i}\right)}\left(\frac{\partial \delta_{i}}{\partial \boldsymbol{\zeta}_{i}}\right)\right\} \\
& \frac{\partial\left[\frac{\left.\bar{r}_{2 i k}-\bar{f}_{2 i k} P_{i k}^{*}\right)}{P_{i k}^{*}\left(1-P_{i k}^{*}\right)}\right]}{\partial \boldsymbol{\zeta}_{i}}=\frac{-\bar{f}_{2 i k} P_{i k}^{*}\left(1-P_{i k}^{*}\right)\left(\partial P_{i k}^{*} / \partial \boldsymbol{\zeta}_{i}\right)-\left(\bar{r}_{2 i k}-\bar{f}_{2 i k} P_{i k}^{*}\right)\left(1-2 P_{i k}^{*}\right)\left(\partial P_{i k}^{*} / \partial \boldsymbol{\zeta}_{i}\right)}{\left[P_{i k}^{*}\left(1-P_{i k}^{*}\right)\right]^{2}} \\
& =\frac{\partial P_{i k}^{*}}{\partial \boldsymbol{\zeta}_{i}}\left\{\frac{-\bar{f}_{2 i k} P_{i k}^{*}-\bar{r}_{2 i k}+2 \bar{r}_{2 i k} P_{i k}^{*}+\bar{f}_{2 i k} P_{i k}^{*}-2 \bar{f}_{2 i k}\left(P_{i k}^{*}\right)^{2}+\bar{f}_{2 i k}\left(P_{i k}^{*}\right)^{2}}{\left[P_{i k}^{*}\left(1-P_{i k}^{*}\right)\right]^{2}}\right\} \\
& =\frac{\partial P_{i k}^{*}}{\partial \boldsymbol{\zeta}_{i}}\left\{\frac{-\left[\bar{r}_{2 i k}-2 \bar{r}_{2 i k} P_{i k}^{*}+\bar{f}_{2 i k}\left(P_{i k}^{*}\right)^{2}\right]}{\left[P_{i k}^{*}\left(1-P_{i k}^{*}\right)\right]^{2}}\right\}
\end{aligned}
$$




$$
\begin{aligned}
& \frac{\partial\left[\frac{\left.\bar{r}_{1 i k}-\bar{f}_{1 i k} P_{i k}\right)}{P_{i k}\left(1-P_{i k}\right)}\right]}{\partial \boldsymbol{\zeta}_{i}}=\frac{-\bar{f}_{1 i k} P_{i k}\left(1-P_{i k}\right)\left(\partial P_{i k} / \partial \boldsymbol{\zeta}_{i}\right)-\left(\bar{r}_{1 i k}-\bar{f}_{1 i k} P_{i k}\right)\left(1-2 P_{i k}\right)\left(\partial P_{i k} / \partial \boldsymbol{\zeta}_{i}\right)}{\left[P_{i k}\left(1-P_{i k}\right)\right]^{2}} \\
& =\frac{\partial P_{i k}}{\partial \boldsymbol{\zeta}_{i}}\left\{\frac{-\bar{f}_{1 i k} P_{i k}-\bar{r}_{1 i k}+2 \bar{r}_{1 i k} P_{i k}+\bar{f}_{1 i k} P_{i k}-2 \bar{f}_{1 i k}\left(P_{i k}\right)^{2}+\bar{f}_{1 i k}\left(P_{i k}\right)^{2}}{\left[P_{i k}\left(1-P_{i k}\right)\right]^{2}}\right\} \\
& =\frac{\partial P_{i k}}{\partial \boldsymbol{\zeta}_{i}}\left\{\frac{-\left[\bar{r}_{1 i k}-2 \bar{r}_{1 i k} P_{i k}+\bar{f}_{1 i k}\left(P_{i k}\right)^{2}\right]}{\left[P_{i k}\left(1-P_{i k}\right)\right]^{2}}\right\} \\
& \frac{\partial\left[\frac{\left(\bar{f}_{2 i k}-\left(\bar{f}_{1 i k}+\bar{f}_{2 i k}\right) \delta_{i}\right)}{\delta_{i}\left(1-\delta_{i}\right)}\right]}{\partial \boldsymbol{\zeta}_{i}}=\frac{-\left(\bar{f}_{1 i k}+\bar{f}_{2 i k}\right) \delta_{i}\left(1-\delta_{i}\right)\left(\partial \delta_{i} / \partial \boldsymbol{\zeta}_{i}\right)-\left(\bar{f}_{2 i k}-\left(\bar{f}_{1 i k}+\bar{f}_{2 i k}\right) \delta_{i}\right)\left(1-2 \delta_{i}\right)\left(\partial \delta_{i} / \partial \boldsymbol{\zeta}_{i}\right)}{\left[\delta_{i}\left(1-\delta_{i}\right)\right]^{2}} \\
& =\frac{\partial \delta_{i}}{\partial \boldsymbol{\zeta}_{i}}\left\{\frac{-\left(\bar{f}_{1 i k}+\bar{f}_{2 i k}\right) \delta_{i}-\bar{f}_{2 i k}+2 \bar{f}_{2 i k} \delta_{i}+\left(\bar{f}_{1 i k}+\bar{f}_{2 i k}\right) \delta_{i}-2\left(\bar{f}_{1 i k}+\bar{f}_{2 i k}\right)\left(\delta_{i}\right)^{2}+\left(\bar{f}_{1 i k}+\bar{f}_{2 i k}\right)\left(\delta_{i}\right)^{2}}{\left[\delta_{i}\left(1-\delta_{i}\right)\right]^{2}}\right\} \\
& =\frac{\partial \delta_{i}}{\partial \boldsymbol{\zeta}_{i}}\left\{\frac{-\left[\bar{f}_{2 i k}-2 \bar{f}_{2 i k} \delta_{i}+\left(\bar{f}_{1 i k}+\bar{f}_{2 i k}\right)\left(\delta_{i}\right)^{2}\right]}{\left[\delta_{i}\left(1-\delta_{i}\right)\right]^{2}}\right\} \\
& \begin{array}{l}
\frac{\partial^{2} E[l(\boldsymbol{\zeta})]}{\partial \boldsymbol{\zeta}_{i} \partial \boldsymbol{\zeta}_{i}^{t}}=\frac{\partial}{\boldsymbol{\zeta}_{i}}\left[\frac{\partial E[l(\boldsymbol{\zeta})]}{\partial \boldsymbol{\zeta}_{i}}\right]^{t} \\
=\frac{\partial}{\boldsymbol{\zeta}_{i}}\left\{\sum _ { k = 1 } ^ { q } \left[\frac{\left(\bar{r}_{2 i k}-\bar{f}_{2 i k} P_{i k}^{*}\right)}{P_{i k}^{*}\left(1-P_{i k}^{*}\right)}\left(\frac{\partial P_{i k}^{*}}{\partial \boldsymbol{\zeta}_{i}}\right)+\right.\right.
\end{array} \\
& \left.\left.\frac{\left(\bar{r}_{1 i k}-\bar{f}_{1 i k} P_{i k}\right)}{P_{i k}\left(1-P_{i k}\right)}\left(\frac{\partial P_{i k}}{\partial \boldsymbol{\zeta}_{i}}\right)-\frac{\bar{f}_{2 i k}-\left(\bar{f}_{2 i k}+\bar{f}_{1 i k}\right) \delta_{i}}{\delta_{i}\left(1-\delta_{i}\right)}\left(\frac{\partial \delta_{i}}{\partial \boldsymbol{\zeta}_{i}}\right)\right]\right\}^{t} \\
& =\sum_{k=1}^{q}\left\{\frac{\left(\bar{r}_{2 i k}-\bar{f}_{2 i k} P_{i k}^{*}\right)}{P_{i k}^{*}\left(1-P_{i k}^{*}\right)}\left(\frac{\partial^{2} P_{i k}^{*}}{\partial \boldsymbol{\zeta}_{i} \partial \boldsymbol{\zeta}_{i}^{t}}\right)+\frac{\partial\left[\frac{\left(\bar{r}_{2 i k}-\bar{z}_{2 i k} P_{i k}^{*}\right)}{P_{i k}^{*}\left(1-P_{i k}\right)}\right]}{\partial \boldsymbol{\zeta}_{i}}\left(\frac{\partial P_{i k}^{*}}{\partial \boldsymbol{\zeta}_{i}}\right)+\right. \\
& \frac{\left(\bar{r}_{1 i k}-\bar{f}_{1 i k} P_{i k}\right)}{P_{i k}\left(1-P_{i k}\right)}\left(\frac{\partial^{2} P_{i k}}{\partial \boldsymbol{\zeta}_{i} \partial \boldsymbol{\zeta}_{i}^{t}}\right)+\frac{\partial\left[\frac{\left(\bar{r}_{1 i k}-\bar{f}_{1 i k} P_{i k}\right)}{P_{i k}\left(1-P_{i k}\right)}\right]}{\partial \boldsymbol{\zeta}_{i}}\left(\frac{\partial P_{i k}}{\partial \boldsymbol{\zeta}_{i}}\right)+ \\
& \left.\frac{\left[\bar{f}_{2 i k}-\left(\bar{f}_{1 i k}+\bar{f}_{2 i k}\right) \delta_{i}\right]}{\delta_{i}\left(1-\delta_{i}\right)}\left(\frac{\partial^{2} \delta_{i}}{\partial \boldsymbol{\zeta}_{i} \partial \boldsymbol{\zeta}_{i}^{t}}\right)+\frac{\partial\left[\frac{\left(\bar{f}_{2 i k}-\left(\bar{f}_{1 i k}+\bar{f}_{2 i k}\right) \delta_{i}\right)}{\delta_{i}\left(1-\delta_{i}\right)}\right]}{\partial \boldsymbol{\zeta}_{i}}\right\} \\
& =\sum_{k=1}^{q}\left\{\frac{\left(\bar{r}_{2 i k}-\bar{f}_{2 i k} P_{i k}^{*}\right)}{P_{i k}^{*}\left(1-P_{i k}^{*}\right)}\left(\frac{\partial^{2} P_{i k}^{*}}{\partial \boldsymbol{\zeta}_{i} \partial \boldsymbol{\zeta}_{i}^{t}}\right)-\right. \\
& \frac{\bar{r}_{2 i k}-2 \bar{r}_{2 i k} P_{i k}^{*}+\bar{f}_{2 i k}\left(P_{i k}^{*}\right)^{2}}{\left[P_{i k}^{*}\left(1-P_{i k}^{*}\right)\right]^{2}}\left(\frac{\partial P_{i k}^{*}}{\partial \boldsymbol{\zeta}_{i}}\right)\left(\frac{\partial P_{i k}^{*}}{\partial \boldsymbol{\zeta}_{i}^{t}}\right)+
\end{aligned}
$$




$$
\begin{aligned}
& \frac{\left(\bar{r}_{1 i k}-\bar{f}_{1 i k} P_{i k}\right)}{P_{i k}\left(1-P_{i k}\right)}\left(\frac{\partial^{2} P_{i k}}{\partial \boldsymbol{\zeta}_{i} \partial \boldsymbol{\zeta}_{i}^{t}}\right)- \\
& \frac{\bar{r}_{1 i k}-2 \bar{r}_{1 i k} P_{i k}+\bar{f}_{1 i k}\left(P_{i k}\right)^{2}}{\left[P_{i k}\left(1-P_{i k}\right]^{2}\right.}\left(\frac{\partial P_{i k}}{\partial \boldsymbol{\zeta}_{i}}\right)\left(\frac{\partial P_{i k}}{\partial \boldsymbol{\zeta}_{i}^{t}}\right)- \\
& \left.\frac{\bar{f}_{2 i k}-2 \bar{f}_{2 i k} \delta_{i}+\left(\bar{f}_{1 i k}+\bar{f}_{2 i k}\right)\left(\delta_{i}\right)^{2}}{\left[\delta_{i}\left(1-\delta_{i}\right)\right]^{2}}\left(\frac{\partial \delta_{i}}{\partial \boldsymbol{\zeta}_{i}}\right)\left(\frac{\partial \delta_{i}}{\partial \boldsymbol{\zeta}_{i}^{t}}\right)\right\}
\end{aligned}
$$

Em que a última igualdade foi obtida a partir de A.39)-A.41). 


\section{Apêndice B}

\section{Escalas Psiquiátricas}

\section{B.1 Inventário de Depressão de Beck (BDI)}

Este questionário consiste em 21 grupos de afirmações. Depois de ler cuidadosamente cada grupo, faça um círculo em torno do número (0, 1, 2 ou 3$)$ diante da afirmação, em cada grupo, que descreve melhor a maneira como você tem se sentido nesta semana, incluindo hoje.

1. 0 Não me sinto triste.

$1 \mathrm{Eu}$ me sinto triste.

2 Estou sempre triste e não consigo sair disso.

3 Estou tão triste ou infeliz que não consigo suportar.

2. 0 Não estou especialmente desanimado quanto ao futuro.

$1 \mathrm{Eu}$ me sinto desanimado quanto ao futuro.

2 Acho que nada tenho a esperar.

3 Acho o futuro sem esperança e tenho a impressão de que as coisas não podem melhorar.

3. 0 Não me sinto um fracasso.

1 Acho que fracassei mais do que uma pessoa comum.

2 Quando olho para trás, na minha vida, tudo o que posso ver é um monte de fracassos.

3 Acho que, como pessoa, sou um completo fracasso.

4. 0 Tenho tanto prazer em tudo como antes.

1 Não sinto mais prazer nas coisas como antes. 
2 Não encontro um prazer real em mais nada.

3 Estou insatisfeito ou aborrecido com tudo.

5. 0 Não me sinto especialmente culpado.

1 Eu me sinto culpado às vezes.

2 Eu me sinto culpado na maior parte do tempo.

3 Eu me sinto sempre culpado.

6. 0 Não acho que esteja sendo punido.

1 Acho que posso ser punido.

2 Creio que vou ser punido.

3 Acho que estou sendo punido.

7. 0 Não me sinto decepcionado comigo mesmo.

1 Estou decepcionado comigo mesmo.

2 Estou enojado de mim.

$3 \mathrm{Eu}$ me odeio.

8. 0 Não me sinto de qualquer modo pior que os outros.

1 Sou crítico em relação a mim devido a minhas fraquezas ou meus erros.

2 Eu me culpo sempre por minhas falhas.

$3 \mathrm{Eu}$ me culpo por tudo de mal que acontece.

9. 0 Não tenho quaisquer idéias de me matar.

1 Tenho idéias de me matar, mas não as executaria.

2 Gostaria de me matar.

$3 \mathrm{Eu}$ me mataria se tivesse oportunidade.

10. 0 Não choro mais que o habitual.

1 Choro mais agora do que costumava.

2 Agora, choro o tempo todo.

3 Costumava ser capaz de chorar, mas agora não consigo mesmo que o queira.

11. 0 Não sou mais irritado agora do que já fui.

1 Fico molestado ou irritado mais facilmente do que costumava.

2 Atualmente me sinto irritado o tempo todo.

3 Absolutamente não me irrito com as coisas que costumavam irritar-me.

12. 0 Não perdi o interesse nas outras pessoas.

1 Interesso-me menos do que costumava pelas outras pessoas.

2 Perdi a maior parte do meu interesse nas outras pessoas.

3 Perdi todo o meu interesse nas outras pessoas.

13. 0 Tomo decisões mais ou menos tão bem como em outra época.

1 Adio minhas decisões mais do que costumava. 
2 Tenho maior dificuldade em tomar decisões do que antes.

3 Não consigo mais tomar decisões.

14. 0 Não sinto que minha aparência seja pior do que costumava ser.

1 Preocupo-me por estar parecendo velho ou sem atrativos.

2 Sinto que há mudanças permanentes em minha aparência que me fazem parecer sem atrativos.

3 Considero-me feio.

15. 0 Posso trabalhar mais ou menos tão bem quanto antes.

1 Preciso de um esforço extra para começar qualquer coisa.

2 Tenho de me esforçar muito até fazer qualquer coisa.

3 Não consigo fazer nenhum trabalho.

16. 0 Durmo tão bem quanto de hábito.

1 Não durmo tão bem quanto costumava.

2 Acordo uma ou duas horas mais cedo do que de hábito e tenho dificuldade para voltar a dormir.

3 Acordo várias horas mais cedo do que costumava e tenho dificuldade para voltar a dormir.

17. 0 Não fico mais cansado que de hábito.

1 Fico cansado com mais facilidade do que costumava.

2 Sinto-me cansado ao fazer quase qualquer coisa.

3 Estou cansado demais para fazer qualquer coisa.

18. 0 Meu apetite não está pior do que de hábito.

1 Meu apetite não é tão bom quanto costumava ser.

2 Meu apetite está muito pior agora.

3 Não tenho mais nenhum apetite.

19. 0 Não perdi muito peso, se é que perdi algum ultimamente.

1 Perdi mais de $2,5 \mathrm{Kg}$.

2 Perdi mais de $5,0 \mathrm{Kg}$.

3 Perdi mais de $7,5 \mathrm{Kg}$.

Estou deliberadamente tentando perder peso, comendo menos: SIM ( ) NÃO ( )

20. 0 Não me preocupo mais que o de hábito com minha saúde.

1 Preocupo-me com problemas físicos como dores e aflições ou perturbações no estômago ou prisão de ventre.

2 Estou muito preocupado com problemas físicos e é difícil pensar em outra coisa que não isso.

3 Estou tão preocupado com meus problemas físicos que não consigo pensar em outra coisa. 
21. 0 Não tenho observado qualquer mudança recente em meu interesse sexual.

1 Estou menos interessado por sexo que costumava.

2 Estou bem menos interessado em sexo atualmente.

3 Perdi completamente o interesse por sexo.

\section{B.2 Escala de Sintomas Depressivos (CES-D)}

Segue abaixo uma lista de tipos de sentimentos e comportamentos. Assinale a frequencia com que tenha se sentido desta maneira durante a semana passada, da seguinte forma: $\mathbf{0}$. Raramente (menos que 1 dia); 1. Durante pouco tempo (1 ou 2 dias); 2. Durante um tempo moderado (3 a 4 dias); 3. Durante a maior parte do tempo (5 a 7 dias)

\begin{tabular}{|c|c|c|c|c|}
\hline & 0 & 1 & 2 & 3 \\
\hline 1. Senti-me incomodado com coisas que habitualmente não me incomodam & & & & \\
\hline 2. Não tive vontade de comer; tive pouco apetite & & & & \\
\hline $\begin{array}{l}\text { 3. Senti não conseguir melhorar meu estado de ânimo mesmo com a ajuda de } \\
\text { familiares e amigos }\end{array}$ & & & & \\
\hline $\begin{array}{l}\text { 4. Senti-me, comparando-me às outras pessoas, tendo tanto valor quanto a } \\
\text { maioria delas }\end{array}$ & & & & \\
\hline 5. Senti dificuldade em me concentrar no que estava fazendo & & & & \\
\hline 6. Senti-me deprimido & & & & \\
\hline 7. Senti que tive que fazer esforço para dar conta das minhas tarefas habituais & & & & \\
\hline 8. Senti-me otimista com relação ao futuro & & & & \\
\hline 9. Considerei que minha vida tinha sido um fracasso & & & & \\
\hline 10. Senti-me amedrontado & & & & \\
\hline 11. Meu sono não foi repousante & & & & \\
\hline 12. Estive feliz & & & & \\
\hline 13. Falei menos que o habitual & & & & \\
\hline 14. Senti-me sozinho & & & & \\
\hline 15. As pessoas não foram amistosas comigo & & & & \\
\hline 16. Aproveitei minha vida & & & & \\
\hline 17. Tive crises de choro & & & & \\
\hline 18. Senti-me triste & & & & \\
\hline 19. Senti que as pessoas não gostavam de mim & & & & \\
\hline 20. Não consegui levar adiante minhas coisas & & & & \\
\hline
\end{tabular}




\section{B.3 Escala de Rastreamento de Dependência de Sexo (ERDS)}

\begin{tabular}{|c|c|c|}
\hline & Sim & Não \\
\hline 1. Você sofreu abuso sexual quando criança ou na adolescência? & & \\
\hline 2. Você tem assinado ou comprado regularmente revistas pornográficas? & & \\
\hline 3. Seus pais tiveram problemas de ordem sexual? & & \\
\hline 4. Você freqüentemente se percebe preocupado com questões sexuais? & & \\
\hline 5. Você acha que seu comportamento sexual não é normal? & & \\
\hline $\begin{array}{l}\text { 6. Sua(seu) esposa(o) ou companheira(o) se procupa ou até mesmo reclama } \\
\text { do seu comportamento sexual? }\end{array}$ & & \\
\hline $\begin{array}{l}\text { 7. Para você é difícil interromper seu comportamento sexual mesmo sabendo } \\
\text { que é inadequado? }\end{array}$ & & \\
\hline 8. Você chega a se sentir mal por causa de sua conduta sexual? & & \\
\hline 9. Sua conduta sexual já causou problemas a você ou à sua família? & & \\
\hline $\begin{array}{l}\text { 10. Você alguma vez já buscou ajuda para lidar com comportamentos sexuais } \\
\text { de que não gostava? }\end{array}$ & & \\
\hline $\begin{array}{l}\text { 11. Você já chegou a se preocupar com o fato de as algumas pessoas desco- } \\
\text { brirem a respeito de suas atividades sexuais? }\end{array}$ & & \\
\hline 12. Alguém já se feriu emocionalmente devido à sua conduta sexual? & & \\
\hline 13. Alguma de suas atividades sexuais é ilegal? & & \\
\hline $\begin{array}{l}\text { 14. Você já se prometeu deixar de fazer alguma coisa relacionada ao seu } \\
\text { comportamento sexual? }\end{array}$ & & \\
\hline $\begin{array}{l}\text { 15. Você já fez alguma tentativa de interromper algum aspecto de sua conduta } \\
\text { sexual e acabou não conseguido? }\end{array}$ & & \\
\hline $\begin{array}{l}\text { 16. Você tem que esconder dos outros algum aspecto de seu comportamento } \\
\text { sexual? }\end{array}$ & & \\
\hline $\begin{array}{l}\text { 17. Você já tentou parar de fazer alguma coisa relacionada a sua atividade } \\
\text { sexual? }\end{array}$ & & \\
\hline 18. Você já achou que o seu comportamento sexual era degradante? & & \\
\hline 19. Sexo é para você alguma forma de escapar de seus problemas? & & \\
\hline 20. Você se sente deprimido após fazer sexo? & & \\
\hline $\begin{array}{l}\text { 21. Você já sentiu necessidade de deixar de praticar alguma forma de compor- } \\
\text { tamento sexual? }\end{array}$ & & \\
\hline 22. Sua atividade sexual interfere na sua vida familiar? & & \\
\hline 23. Você já manteve práticas sexuais com menores de idade? & & \\
\hline 24. Você sente que é controlado por seu desejo sexuai? & & \\
\hline 25. Você sente que seu desejo sexual é mais forte do que você? & & \\
\hline
\end{tabular}




\title{
B.4 Teste de Investigação Bulímica de Edinburgh (TIBE)
}

\author{
PARTE I $(\operatorname{sim}=1$ e não $=0)$
}

1. Qual a sua altura?

2. Qual o seu peso atual?

3. Qual o peso máximo que você já apresentou?

4. Qual o peso mínimo que você já apresentou?

5. Qual é no seu entender, seu peso ideal?

6. Você se sente em relação a seu peso:

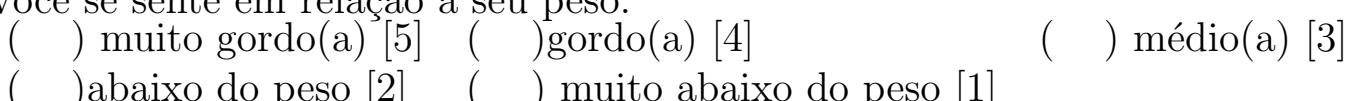

7. Você tem períodos menstruais regulares? Não ( ) Sim ( )

8. Com que freqüência semanal você faz as seguintes refeições?

\begin{tabular}{|l|c|c|c|c|c|}
\hline & 7 dias & 5 dias & 3 dias & 1 dia & nunca \\
\hline Café da manhã & 1 & 2 & 3 & 4 & 5 \\
\hline Almoço & 1 & 2 & 3 & 4 & 5 \\
\hline Jantar & 1 & 2 & 3 & 4 & 5 \\
\hline Lanche entre as refeições & 1 & 2 & 3 & 4 & 5 \\
\hline
\end{tabular}

9. Você alguma vez teve uma orientação profissional com a finalidade de fazer regime ou ser orientado (a) quanto à sua alimentação? Não( ） Sim ( )

10. Alguma vez foi membro de alguma sociedade ou clube para emagrecimento?

Não ( ) Sim ( )

11. Você alguma vez teve algum tipo de problema alimentar? Não ( ) Sim ( )

12. Caso sim descreva com detalhes:

\section{PARTE II $(\operatorname{sim}=1$ e não=0)}

1. Você tem um padrão de alimentação diário regular? Não $(\quad$ ) Sim $(\quad$ )

2. Você segue uma dieta rígida? Não ( ) Sim ( )

3. Você sente-se fracassado quando quebra sua dieta uma vez? Não $(\quad$ ) Sim $(\quad)$

4. Você conta as calorias de tudo que come, mesmo quando não está de dieta?

Não ( ) Sim ( )

5. Você já jejuou por um dia inteiro? Não ( ) Sim ( )

6. Se sim qual a freqüência?

( ) dias alternados [5] ( ) 2 a 3 vezes por semana [4] ( ) 1 vez por semana [3]

( )de vez em quando [2] ( ) somente $1 \mathrm{vez}[1]$

7. Você usa uma das seguintes estratégias para auxiliar na sua perda de peso? 


\begin{tabular}{|l|l|l|l|l|l|l|l|}
\hline & & & \multicolumn{3}{|c|}{$N^{o}$ de vezes na semana } & \multicolumn{2}{l|}{$N^{o}$ de vezes no dia } \\
\hline & nunca & às vezes & 1 & 2 a 3 & 7 & 2 a 3 & 5 ou + \\
\hline Tomar comprimidos & 0 & 2 & 3 & 4 & 5 & 6 & 7 \\
\hline Tomar diuréticos & 0 & 2 & 3 & 4 & 5 & 6 & 7 \\
\hline Tomar laxantes & 0 & 2 & 3 & 4 & 5 & 6 & 7 \\
\hline Vômitos & 0 & 2 & 3 & 4 & 5 & 6 & 7 \\
\hline
\end{tabular}

8. O seu padrão de alimentação prejudica seriamente sua vida? Não ( ） Sim ( )

9. Você poderia dizer que a comida dominou sua vida? Não $(\quad) \operatorname{Sim}(\quad)$

10. Você come sem parar até ser obrigado(a) a parar por sentir-se mal fisicamente?

Não ( ) Sim ( )

11. Há momentos em que você só consegue pensar em comida? Não $(\quad$ ) Sim $(\quad$ )

12. Você come moderadamente na frente dos outros e depois exagera em particular?

Não ( ) Sim ( )

13. Você sempre pode parar de comer quando quer? Não $(\quad)$ Sim $(\quad$ )

14. Você já sentiu incontrolável desejo para comer sem parar? Não $(\quad$ ) Sim ( )

15. Quando você se sente ansioso(a), você tende a comer muito? Não ( ) Sim ( )

16. O pensamento de tornar-se gordo(a) o(a) apavora? Não ( ) Sim ( )

17. Você já comeu grandes quantidades de comida muito rapidamente (não uma refeição)?

Não ( ) Sim ( )

18. Você se envergonha de seus hábitos alimentares? Não ( ） Sim ( )

19. Você preocupa - se com fato de não ter controle sobre o quanto você come?

Não ( ) Sim ( )

20. Você volta-se para comida para aliviar algum tipo de desconforto?

Não ( ) Sim ( )

21. Você é capaz de deixar comida no prato ao final de uma refeição? Não $(\quad$ ） Sim ( )

22. Você engana os outros sobre quanto come? Não ( ) Sim ( )

23. O quanto você come é determinado pela fome que você sente? Não $(\quad$ ） Sim ( )

24. Você já teve episódios exagerados de alimentação? Não ( ） Sim ( ）

25. Se sim esses episódios deixaram você sentindo-se mal? Não $(\quad) \operatorname{Sim}(\quad)$

26. Se você tem esses episódios, eles ocorrem só quando você está sozinho(a)?

Não ( ) Sim ( )

27. Se você tem episódios qual a sua freqüência?

( ) 2 a 3 vezes por dia [6] ( ) diariamente [5] ( )2 a 3 vezes por semana [4]

( ) 1 vez por semana [3] （ )1 vez por mês [2] （）quase nunca [1]

28. Você iria até as últimas conseqüências para satisfazer um desejo de alimentação exagerado? Não ( ) Sim ( )

29. Se você come demais você sente- se muito culpado(a)? Não $(\quad) \operatorname{Sim}(\quad)$

30. Você já comeu escondido(a)? Não ( ) Sim ( )

31. Seus hábitos alimentares são o que você poderia considerar normais?

Não ( ) Sim ( )

32. Você considera-se alguém que come compulsivamente? Não $(\quad$ ) Sim $(\quad)$

33. Seu peso flutua mais que $2,5 \mathrm{Kg}$ em uma semana? Não （） Sim （） 


\section{B.5 Inventário de Triagem do Uso de Drogas Refor- mulado (DUSI-R)}

Por favor, responda "SIM" ou "NÃO"para todas as questões a seguir. Responda as questões considerando o que ocorreu nos últimos 12 meses. Caso alguma questão não se aplique a você, responda "NÃO".

\section{Área I}

1. Você já teve "fissura" ou um forte desejo por álcool ou drogas? _-_- 2. Você já teve que usar mais e mais drogas ou álcool para conseguir o efeito desejado? 3. Você já sentiu que não poderia controlar o uso de álcool ou drogas? --_-- 4. Você já sentiu que estava "enfeitiçado" ou muito envolvido pelo álcool ou pelas drogas? ----- 5. Você já deixou de realizar alguma atividade por ter gasto muito dinheiro com drogas ou álcool? 6. Você já quebrou regras ou desobedeceu leis por estar "alto" sob o efeito de álcool ou drogas? 7. Você muda rapidamente de muito feliz para muito triste ou de muito triste para muito feliz, por causa das drogas? 8. Você já sofreu algum acidente de carro depois de usar álcool ou drogas? 9. Você já se machucou acidentalmente ou machucou alguém depois de usar álcool ou drogas? 10. Você já teve uma discussão séria ou briga com um amigo ou membro da família por causa da bebida ou do seu uso de drogas? ----- 11. Você já teve problemas em se dar bem com algum de seus amigos devido ao uso de álcool ou drogas? __-_ 12. Você já teve sintomas de abstinência após o uso de álcool? (Por exemplo: dor de cabeça, náuseas, vômitos ou tremores) _-__ 13. Você já teve problemas para lembrar o que fez enquanto estava sob efeito de drogas ou álcool? _-_-- 14. Você gosta de brincadeiras que envolvem bebidas quando vai a festas? (Por exemplo: "vira-vira"; apostas para ver quem bebe mais rápido ou em maior quantidade; etc.) -_--- 15. Você tem problemas para resistir ao uso de álcool ou drogas? 16. Alguma vez você já disse uma mentira?

\section{Área II}

1. Você briga muito? 2. Você se acha o "bom"? 3. Você provoca ou faz coisas prejudiciais aos animais? 4. Você grita muito? 5. Você é teimoso? 6. Você é 
desconfiado em relação a outras pessoas? 7. Você pragueja (reclama muito) ou fala muitos palavrões? _-_- 8. Você provoca muito as pessoas? __-_ 9. Você tem um temperamento difícil? 10. Você é muito tímido? _---- 11. Você ameaça ferir as pessoas? --_-- 12. Você fala mais alto que os outros jovens? 13. Você se chateia (ou se aborrece) facilmente? 14. Você faz muitas coisas sem primeiro pensar nas conseqüências? 15. Você se arrisca ou faz coisas perigosas muitas vezes? 16. Se tiver oportunidade você tira vantagem das pessoas? 17. Geralmente você se sente irritado? _-_-- 18. Você gasta a maior parte do tempo livre sozinho? 19. Você é um solitário? 20. Você é muito sensível a críticas? 21. Seus modos de comer são melhores nos restaurante do que em casa?

\section{Área III}

1. Você se submeteu a algum exame físico ou esteve sob cuidados médicos no ano passado?

2. Você teve algum acidente ou ferimento que ainda o incomode?

3. Você dorme demais ou muito pouco? 4. Recentemente, você perdeu ou ganhou mais de $4 \mathrm{~kg}$ ? 5. Você tem menos energia do que acha que deveria ter? 6. Você tem problemas de respiração ou de tosse? _-_-- 7. Você tem alguma preocupação sobre sexo ou com seus órgãos sexuais? _-_-_ 8. Você já teve relações sexuais com alguém que usava drogas injetáveis? 9. Você teve dores abdominais ou náuseas no ano passado? 10. A parte branca de seus olhos já ficou amarela?

11. Você às vezes sente vontade de xingar?

\section{Área IV}

1. Intencionalmente, você já danificou a propriedade de alguém? 2. Você já roubou coisas em mais de uma ocasião? 3. Você se envolveu em mais brigas que a maioria dos jovens? ---- 4. Você é uma pessoa inquieta? 5. Você é agitado e não consegue sentar quieto? 6. Você fica frustrado facilmente? 7. Você tem problemas em se concentrar? 8. Você se sente muito triste? 9. Você rói unhas? 10. Você tem problemas para dormir?

11. Você é nervoso? 12. Você se sente facilmente amedrontado? 13. Você se preocupa demais? 14. Você tem dificuldade em deixar de pensar em determinadas coisas? 15. As pessoas olham espantadas para você? 16. Você escuta coisas que ninguém mais do seu lado escuta? 17. Você tem poderes especiais que ninguém mais tem? 18. Você sente medo de estar entre as pessoas? --_-- 19. Você freqüentemente sente vontade de chorar? _-_--- 20. Você tem tanta energia que não sabe o que fazer consigo mesmo? 21. Alguma vez você se sentiu tentado a roubar alguma coisa? 


\section{Área V}

1. Os jovens de sua idade não gostam de você? 2. Você está normalmente infeliz com o modo como desempenha atividades com seus amigos?

3. É difícil fazer amizades num grupo novo? 4. As pessoas tiram vantagens de você? 5. Você tem medo de lutar pelos seus direitos?

6. É difícil para você pedir ajuda aos outros? 7. Você é facilmente influenciado por outros jovens? 8. Você prefere ter atividades com jovens bem mais velhos que você?

9. Você se preocupa em como suas ações vão afetar os outros? 10. Você tem dificuldades em defender suas opiniões? 11. Você tem dificuldade em dizer "não" para as pessoas?

12. Você se sente desconfortável (sem jeito) se alguém o elogia? 13. As pessoas o enxergam como uma pessoa não amigável? 14. Você evita olhar nos olhos quando está conversando com as pessoas? 15. O seu humor às vezes muda?

\section{Área VI}

1. Algum membro de sua família (mãe, pai, irmão ou irmã) já usou maconha ou cocaína?

2. Algum membro de sua família usou álcool a ponto de causar problemas em casa, no trabalho ou com amigos? _---- 3. Algum membro de sua família já foi preso? 4. Você tem discussões freqüentes com seus pais ou responsáveis que envolvam gritos e berros? 5. Sua família dificilmente faz coisas juntas? 6. Seus pais ou responsáveis desconhecem o que você gosta e o que não gosta? 7. Na sua casa faltam regras claras sobre o que você pode e não pode fazer? _-_ 8. Seus pais ou responsáveis desconhecem o que você realmente pensa ou sente sobre as coisas que são importantes para você? 9. Seus pais ou responsáveis brigam muito entre si? --_-- 10. Seus pais ou responsáveis freqüentemente desconhecem onde você está ou o que você está fazendo? ----- 11. Seus pais ou responsáveis estão fora de casa a maior parte do tempo? 12. Você sente que seus pais ou responsáveis não se importam ou não cuidam de você? 13. Você se sente infeliz em relação ao modo como você vive? 14. Você se sente em perigo em casa? 15. Você às vezes fica bravo?

\section{Área VII}

1. Você não gosta da escola? 2. Você tem problemas para se concentrar na escola ou quando está estudando? 3. Suas notas são abaixo da média? 4. Você "cabula" aulas mais de dois dias por mês? 5. Você falta muito à escola? 6. Você já pensou seriamente em 
abandonar a escola?

7. Freqüentemente, você deixa de fazer os deveres escolares?

8.

Você sempre se sente sonolento na aula?

9. Freqüentemente, você chega atrasado para a aula? ----- 10. Nesse ano, seus amigos da escola são diferentes daqueles do ano passado? ----- 11. Você se sente irritado e chateado quando está na escola? 12. Você fica entediado na escola? -_-- 13. Suas notas na escola estão piores do que costumavam ser? 14. Você se sente em perigo na escola? _-_-- 15. Você já repetiu de ano alguma vez? 16. Você se sente indesejado nos clubes escolares (centro acadêmico, atlética, etc.) ou nas atividades extracurriculares?

17. Você já faltou ou chegou atrasado na escola em conseqüência do uso de álcool ou drogas? 18. Você já teve problemas na escola por causa do álcool ou das drogas? 19. O álcool ou drogas já interferiram nas suas lições de casa ou atividades escolares? 20. Você já foi suspenso? 21. Você às vezes adia coisas que você precisa fazer?

\section{Área VIII}

1. Você já teve um trabalho remunerado do qual foi despedido? 2. Você já parou de trabalhar simplesmente porque não se importava? 3. Você precisa de ajuda dos outros para arranjar emprego?

4. Você freqüentemente falta ou chega atrasado no trabalho? 5. Você acha difícil concluir tarefas no seu trabalho? 6. Você já ganhou dinheiro realizando atividades ilegais? 7. Você já consumiu álcool ou drogas durante o trabalho? 8. Você já foi demitido de um emprego por causa de drogas? 9. Você tem problemas de relacionamento com seus chefes? 10. Você trabalha principalmente porque isso permite ter dinheiro para comprar drogas? 11. Você fica mais feliz quando você ganha do que quando você perde em um jogo?

\section{Área IX}

1. Algum de seus amigos usa álcool ou drogas regularmente? _-_-_ 2. Algum de seus amigos vende ou dá drogas a outros jovens? 3. Algum de seus amigos "cola" nas provas? 4. Seus pais ou responsáveis não gostam de seus amigos? 5. Algum dos seus amigos já teve problemas com a lei? 6. A maioria dos seus amigos é mais velha que você? 7. Seus amigos cabulam muitas aulas? 8. Seus amigos ficam entediados nas festas quando não é servido álcool? 9. Seus amigos levaram drogas ou álcool nas festas no ano passado?

10. Seus amigos roubaram alguma coisa de uma loja ou danificaram a propriedade escolar de propósito durante o ano passado? 11. Você pertence a alguma "gang"? 12. Você se 
sente incomodado por problemas que esseja tendo com amigos atualmente? 13. Você sente que não tem nenhum amigo em quem você possa confiar? _-_-- 14. Se comparado com a maioria dos jovens, você tem poucos amigos? ----- 15. Alguma vez você foi convencido a fazer alguma coisa que você não queria fazer?

\section{Área X}

1. Comparado com a maioria dos jovens, você faz menos esportes? _-_-- 2. Durante a semana, você normalmente sai à noite para se divertir, sem permissão? 3. Num dia típico de verão você assiste à televisão por mais de duas horas? 4. Na maioria das festas que você tem ido recentemente, os pais estão ausentes? 5. Você se exercita menos que a maioria dos jovens que você conhece? --_-- 6. Nas suas horas livres você simplesmente passa a maior parte do tempo com os amigos? _-_-- 7. Você se sente entediado a maior parte do tempo? _-_-- 8. Você realiza a maior parte das atividades de lazer sozinho? __-_ 9. Você usa álcool ou drogas para se divertir? ---- 10. Comparado à maioria dos jovens, você está menos envolvido em "hobbies" ou outros interesses? _-__- 11. Você está insatisfeito com a maneira como passa seu tempo livre? _-_-- 12. Você se cansa muito rapidamente quando faz algum esforço físico? 13. Você alguma vez comprou alguma coisa que você não precisava? 


\section{Referências Bibliográficas}

Andrade, D. F., Tavares, H. R. e Valle, R. C. (2000). Teoria de resposta ao item: conceitos e aplicações, Associação Brasileira de Estatística, São Paulo.

Andrich, D. (1978a). Application of a psychometric model to ordered categories which are scored with successive integers, Applied Psychological Measurement 2: 581-594.

Andrich, D. (1978b). A rating formulation for ordered response categories, Psychometrika 43: $561-573$.

Baker, F. B. e Kim, S. (2004). Item Response Theory - Parameter Estimation Techniques, 2nd edn, Marcel Dekker, Inc, New York.

Bartle, R. G. (1966). The Elements of Integration, John Wiley and Sons, New York.

Bock, R. D. (1972). Estimating item parameters and latent ability when responses are scored in two or more nominal categories, Psychometrika 37: 29-51.

Bock, R. D. e Aitkin, M. (1981). Marginal maximum likelihood estimation of item parameters: an application of a em algorithm, Psychometrika 46: 443-459.

Bock, R. D. e Lieberman, M. (1970). Fiting a response model for n dichotomously scored items, Psychometrika 35: 179-197.

Camilli, G. L. e Shepard, L. A. (1994). Methods for identifying biased test items, Thousand Oakes, CA:Sage.

Cappaliez, P. (1989). Social desirability response set and self-report depression inventories in the elderly, Clinical Gerontologist 9: 45-52.

Carmines, E. G. e Zeller, R. A. (1979). Reliability and validity assessment, Sage University Paper series on Quantitative Applications in the Social Sciences, 07-001, Sage Publications, Inc, London. 
Coleman, M. J., Matthysse, S., Levy, D. L., Cook, S., Lo, J. B. Y., Rubin, D. B. e Holzman, P. S. (2002). Spatial and object working memory impairments in schizophrenia patients: a bayesian item-response theory analysis, Journal of Abnormal Psychology 111: 425-435.

Embretson, S. E. e Reise, S. P. (2000). Item response theory for psychologists, Lawrence Erlbaum Associates, Inc., Publishers, New Jersey.

Gardner, W., Kelleher, K. J. e Pajer, K. A. (2000). Multidimensional adaptative testing for mental health problems in primary care, Medical Care 40: 812-823.

Gelman, A. e Rubin, D. B. (1992). Inference for iterative simulation using multiple sequences, Statistical Science 7: 457-511.

Gorenstein, C., Andrade, L. H. S. G. e Zuardi, A. W. (1999). Escalas de avaliação clínica em psiquiatria e psicofarmacologia, Lemos Editorial, São Paulo.

Hambleton, R. K., Swaminathan, H. e Rogers, H. J. (1991). Fundamentals of item response theory, Sage Publications, London.

Hays, R., Morales, L. S. e Reise, S. P. (2000). Item response theory and health outcomes measurement in the $21^{\text {st }}$ century, Medical Care $\mathbf{3 8}$.

Hildebrand, F. B. (1956). Introduction to Numerical Analysis, McGraw-Hill.

Kessler, R. C., Andrews, G., Colpe, L. J., Hiripi, E., Mroczek, D. K., Normand, S. L. T., Walters, E. E. e Zaslavsky, A. M. (2002). Short screening scales to minitor population prevalences and trends in non-specific psychological, Psychological Medicine 32: 959-976.

Kirisci, L., Hsu, T. C. e Tarter, R. (1994). Fitting a two-parameter logistic item response model to clarify the psychometric properties of the drug use screening inventory for adolescent alcohol and drug abusers, Alcohol Clin. Exp. Res 18: 1335-1341.

Langenbucher, J. W., Labouvie, E., Sanjuan, P. M., Bavly, L., Martin, C. S. e Kirisci, L. (2004). An application of item response theory analysis to alcohol, cannabis and cocaine criteria in DSM-IV, Journal of Abnormal Psychology 113: 72-80.

Li, Y. H. e Lissitz, R. W. (2000). An evaluation of the accuracy of multidimensional irt linking, Applied Psychological Measurement 24: 115-138.

Linden, W. J. v. d. e Hambleton, R. K. (1996). Handbook of modern item response theory, Springer, New York. 
Masters, G. N. (1982). A rasch model for partial credit scoring, Psychometrika 47: 149-174.

Muraki, E. (1992). A generalized partial credit model: Application of an em algorithm, Applied Psychological Measurement 16: 159-176.

Nojosa, R. T. (2002). Teoria da reposta ao item - modelos multidimensionais, Estudos de Avaliação Educacional $\mathbf{2 5}$.

Patz, R. J. e Junker, B. W. (1999). A straightforward approach to markov chain monte carlo methods for item response theory models, Journal of Educational and Behavioral Statistics 24: $146-178$.

Pedhazur, E. J. e Schmelkin, L. P. (1991). Measurement, design, and analysis: an integrated approach, Lawrence Erlbaum Associates, New Jersey.

Rasch, G. (1960). Probabilistic Models for Some Intelligence and Attainment Tests, Danish Institute for Educational Research, Copenhagen.

Reckase, M. D. (1997). The past and the future of multidimensional item response theory, Applied Psychological Measurement 21: 25-36.

Ross, S. M. (1997). Simulation, Academic Press, San Diego.

Rost, J. e Carstensen, C. H. (2002). Multidimensional rasch measurement via item component models and faceted designs, Applied Psychological Measurement 26: 42-56.

Samejima, F. (1969). Estimation of latent ability using a response pattern of graded scores, Psychometrika Monograph Supplement 34(17): 1-100.

SAS Institute Inc. (1999). SAS/IML User's Guide, SAS Institute Inc., Cary, NC.

Schaeffer, N. C. (1988). An application of item response theory to the measurement of depression, Sociological Methodology 18: 271-307.

Sen, P. K. e Singer, J. M. (1993). Large Sample Methods in Statistics: An Introduction with Applications, Chapman \& Hall, New York.

Spiegelhalter, D., Thomas, A., Best, N. e Lunn, D. (2003). WinBUGS User Manual Version 1.4, MRC Biostatistics Unit, Cambridge.

Takane, Y. e Leeuw, J. (1988). On the relationship between item response theory and factor analysis of discretized variables, Psychometrika 52: 393-408. 
Wang, Y. P., Andrade, L. H. e Gorenstein, C. (2005). Validation of the beck depression inventory for a portuguese-speaking chinese community in brazil, Brazilian Journal of Medical and Biology Research 38: 399-408.

Ware, J. E., Bjomer, J. B. e Kosinski, M. (2000). Practical implications of item response theory and computerized adaptative testing: A brief summary of ongoing studies of widely use headache impact scales, Medical Care 38(9): 73-82.

Weiss, D. J. (1985). Adaptative testing by computer, Journal of Consulting and Clinical Psychology 53: 774-789. 